\title{
A SMALL LOW ENERGY CYCLOTRON FOR RADIOISOTOPE MEASUREMENTS
}

\author{
Kirk Joseph Bertsche \\ Ph.D. Thesis \\ Department of Physics \\ University of California, Berkeley \\ Berkeley, CA 94720 \\ and \\ Physics Division \\ Lawrence Berkeley Laboratory \\ One Cyclotron Road, Berkeley, CA 94720
}

November, 1989 


\title{
A SMALL LOW ENERGY CYCLOTRON FOR RADIOISOTOPE MEASUREMENTS
}

by

Kirk Joseph Bertsche

\begin{abstract}
Direct detection of ${ }^{14} \mathrm{C}$ by accelerator mass spectrometry has proved to be a much more sensitive method for radiocarbon dating than the decay counting method invented earlier by Libby. A small cyclotron (the "cyclotrino") was proposed for direct detection of radiocarbon in 1980. This combined the suppression of background through the use of negative ions, which had been used effectively in tandem accelerators, with the high intrinsic mass resolution of a cyclotron. Development of a small electrostatically-focused cyclotron for use as a mass spectrometer was previously reported but the sensitivity needed for detection of ${ }^{14} \mathrm{C}$ at natural abundance was not achieved.

The major contributions of this work are the integration of a high current external ion source with a small flat-field, electrostatically-focused cyclotron to comprise a system capable of measuring ${ }^{14} \mathrm{C}$ at natural levels, and the analysis of ion motion in such a cyclotron, including a detailed analysis of phase bunching and its effect on mass resolution. A high current cesium sputter negative ion source generates a beam of carbon ions which is pre-separated with a Wien filter and is transported to the cyclotron via a series of electrostatic lenses. Beam is injected radially into the cyclotron using electrostatic deflectors and an electrostatic mirror. Axial focusing is entirely electrostatic. A microchannel plate detector is used with a phase-gated output.

In its present form the system is capable of improving the sensitivity of detecting ${ }^{14} \mathrm{C}$ in some biomedical experiments by a factor of $10^{4}$. Modifications are discussed which could bring about an additional factor of 100 in sensitivity, which is important for archaeological and geological applications. Possibilities for reasurements of other isotopes, such as ${ }^{3} \mathrm{H}$, and ${ }^{10} \mathrm{Be}$, and ${ }^{26} \mathrm{Al}$, are discussed.
\end{abstract}




\section{AChNOWLEDGEMENTS}

A great number of people have contributed to this project. I am very thankful for their help and wish to acknowledge their contributions. It is perhaps inevitable that some of the many contributors may be overlooked; for this I apologize.

I wish to thank my adviser, Prof. Richard Muller, for the conception and support of this project, Dr. Jim Welch for the hands-on training he provided in development of an earlier version of the cyclotrino, Dr. Peter Friedman for development of the detector which was used, Dr. Pieter Tans, Dr. Don Morris and the late Prof. Luis Alvarez for their helpful advice in the early phases of the project, and Hung Vo, Keith Geller, Chandu Karadi, Matt O'Keefe and Gabriel Paulson for much valuable laboratory assistance.

Many individuals with expertise in a wide variety of disciplines have contributed to making this project a success. I would like to thank Dr. Dave Clark for sharing his expertise in cyclotron design, Dr. Klaus Halbach for expert assistance in magnet design, Dr. Dennis Moltz for advice regarding ion optics, Dr. Ka-ngo Leung, Dr. Steve Walther, Dr. Ken Purser and Prof. Roy Middleton for advice regarding ion sources, Robbie Smits, John Gibson and Dr. Jordin Kare for helpful discussions of electronics problems, Don Landis and Norm Madden for assistance with detection electronics, Dr. Tony Sargent and Prof. Tom Budinger for discussions of biomedical applications for the cyclotrino, Prof. Erv Taylor for encouragement and providing of samples, Dr. Frank Asaro and Dr. Helen Michel for providing enriched graphite, Dr. Doug Donahue for advice regarding graphite samples. John Wool for expert welding, John Meneghetri for advice in mechanical design, Dave Plate and Egon Hoyer for providing samarium cobalt magnet material, Armi Meut for machining, Bill Wilke for ceramics work, Peter Bokavich for providing good magnet-grade steel, Kaarlo Heiskanen for assistance in evaluation of integrals, and Faye Mitschang for valuable administrative help.

I am grateful for the support of Dr. Keith Clemenger, Jim Kintner, Edgar Yep, Steve Rein and Mark Kral, apartment-mates who have provided encouragement during this work. I am especially thankful for the encouragement, prayers and support of my parents, sisters, and sisters' fanilies during this long project, and for the encouragement and prayers of my many friends at Calvary Bible Church, Columbia Presbyterian Church, Sun Valley Bible Chapel and Fairhaven Bible Chapel. 
Above all, I thank my Lord and Savior, Jesus Christ, for His enablement and suidance throughout this project, and for creating such an interesting universe to explore. As J.J. Thompson, the discoverer of the electron, has said, "In the distance tower still higher peaks, which will yield to those who ascend them still wider prospects, and deepen the feeling, the truth of which is emphasized by every advance in science, that 'Great are the Works of the Lord'." (Thompson, 1909)

This work has been supported by the U.S. Department of Energy under contract DE-AC03-76SF00098. 


\section{CONTENTS}

Abstract

iii

Acknowledgements

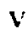

1. INTRODUCTION 1

1.1 Radiocarbon 1

1.2 Accelerator Mass Spectrometry 1

2. CYCLOTRON DESIGN 5

2.1 General Design Considerations 5

2.2 Energy Gain 6

2.3 Stability 11

2.3.1 Axial Stability $\quad 12$

$\begin{array}{ll}2.3 .2 \text { Radial Stability } & 15\end{array}$

$\begin{array}{ll}\text { 2.3.3 Phase Stability } & 17\end{array}$

$\begin{array}{ll}2.4 \text { Acceptance } & 27\end{array}$

3. ION PRODUCTION AND INJECTION 33

3.1 Overview 33

3.2 Ion Source $\quad 37$

3.3 Einzel Lens 38

3.4 Quadrupole Lenses $\quad 39$

3.5 Wien Filter 43

3.6 Injection 49

4. ION EXTRACTION AND DETECTION 53

4.1 Extractor 53

4.2 Detector 53

4.3 Detection Electronics $\quad 54$

5. SYSTEM PERFORMANCE

5.1 Transmission $\quad 57$

$5.2{ }^{14} \mathrm{C}$ Measurements $\quad 59$

6. APPLICATIONS AND IMPROVEMENTS 63

7. CONCLUSIONS 65

$\begin{array}{ll}\text { APPENDICES } & 67\end{array}$

Appendix A: Some Isotopes of Potential Interest 67

Appendix B: Evaluation of Integrals $\quad 69$ 
B.1 Energy Gain $\quad 69$

B.2 Axial Focusing $\quad 70$

B.3 Phase Bunching $\quad 72$

Appendix C: Electronics $\quad 77$

$\begin{array}{ll}\text { C.I RF Circuitry } & 77\end{array}$

C.2 Magnetic Field Measurement $\quad 80$

C.3 High Voltage Circuitry $\quad 80$

$\begin{array}{ll}\text { C.4 Power Supplies } & 84\end{array}$

C.5 Low Current Circuitry 90

C.6 Other Circuitry $\quad 93$

Appendix D: Ion Optical Calculations 95

D.1 Ion Optics Overview $\quad 95$

D.2 First Order Calculations 95

D.3 Large Velocity Deviations 101

D.4 A Practical Wien Filter 103

D.5 Adjustment of Wien Filter Focus 105

Appendix E: Catalog of Matrices for Ion Optical Elements 109

$\begin{array}{ll}\text { Appendix F: Beam Bunching } & 113\end{array}$

F.1 Introduction $\quad 114$

F.2 Simple and Gap Bunchers $\quad 116$

F.3 Design of a Simple Buncher $\quad 119$

F.4 Conclusions 137

F.5 References 137

$\begin{array}{ll}\text { F.6 Acknowledgements } & 137\end{array}$

Appendix G: Counting Rates and Sample Sizes $\quad 139$

$\begin{array}{ll}\text { G.1 Errors } & 139\end{array}$

G.2 Counting Rates 140

G.3 Sample Sizes $\quad 142$

G.4 Tracer Studies 142

$\begin{array}{ll}\text { REFERENCES } & 143\end{array}$ 


\section{INTRODUCTION}

\subsection{Radiocarbon}

Radiocarbon $\left({ }^{14} \mathrm{C}\right)$ is present in modern, living organisms at a concentration of about $10^{-12}$ as compared to the predominant carbon isotope, ${ }^{12} \mathrm{C}$. This level is kept relatively constant due to the large reservoir of carbon present in the earth's biosphere, which acts to buffer any changes in concentration. Once an organism dies carbon is no longer exchanged with the biosphere. The ${ }^{14} \mathrm{C}$ concentration begins to drop through beta decay of ${ }^{14} \mathrm{C}$ which has a half-life of 5730 years. Thus, measurement of the concentration of ${ }^{14} \mathrm{C}$ in a dead organism can be used to calculate the age of the organism.

Because of the very low concentration of ${ }^{14} \mathrm{C}$ even in modem samples, ${ }^{14} \mathrm{C}$ is also useful as a tracer. This has been especially helpful in biological studies. Chemical compounds can be synthesized with ${ }^{14} \mathrm{C}$ rather than ${ }^{12} \mathrm{C}$; these "tagged" compounds are introduced into the organism to be studied. Tracking ${ }^{14} \mathrm{C}$ through the organism allows the study of metabolic and other processes.

Since the introduction of radiocarbon dating by Libby in the 1950 's, ${ }^{14} \mathrm{C}$ measurements, whether for archaeological, biological or other studies, have primarily been done by decay counting (Taylor, 1987). Samples are introduced into a counting chamber where the beta particles ( $156 \mathrm{keV}$ endpoint energy) due to disintegration of ${ }^{14} \mathrm{C}$ are detected with standard nuclear counting apparatus. This method works well for samples which are large enough and recent enough to give a high decay rate (one gram of modern carbon produces about 14 disintegrations per minute [dpm]), but for old or small samples the necessary counting time becomes impractical.

\subsection{Accelerator Mass Spectrometry}

This limitation of decay counting has been answered by accelerator mass spectrometry (AMS). Rather than crounting decays of ${ }^{14} \mathrm{C}$, the ${ }^{14} \mathrm{C}$ atoms themselves are detected and counted using a particle accelerator. This provides a huge advantage in sensitivity over decay counting techniques.

An accelerator was first used as a mass spectrometer by Alvarez when a large cyclotron was used for detection of ${ }^{3} \mathrm{He}$, reported in 1939. (Alvarez and Comog, 1939a,b; 
Alvarez, 1981) The technique was re-introduced by Alvarez in the mid-1970's as part of a search for anomalous particles (quarks) with integral charge (Muller et al., 1977; Muller 1987). It was first suggested as a means of measuring radioisotopes by Muller, who made a successful age determination using tritium (1976). Although early work was done with cyclotrons, measurements quickly shifted to tandem Van de Graaff accelerators, especially for ${ }^{14} \mathrm{C}$ measurements due to the elimination of interference from ${ }^{14} \mathrm{~N}$ (Nelson, Korteling and Stott, 1977; Bennett et al., 1977, 1978; Gove, Litherland and Purser, 1987). During the past ten years or so, techniques have been refined so that AMS is now a quite accurate method of dating ${ }^{14} \mathrm{C}$ and other radioisotopes.

Unfortunately, this method has been relatively costly because of the need for a large, high-energy particle accelerator. In response to the increasing demand and high cost of accelerator mass spectrometry (AMS) techniques, the small cyclctron ("cyclotrino") project was begun at Berkeley in 1981 (Muller et al., 1981). The basic concept was to combine the excellent properties of a cyclotron used as a mass spectrometer with the capabilities of negative ion sources to reject unwanted backgrounds such as ${ }^{14} \mathrm{~N}$ (Nelson, Korteling and Stott, 1977; Bennett et al., 1977).

A small cyclotron (the "cyclotrino") was built at Berkeley to test these concepts (Welch, 1984a; Welch et al., 1984b, 1987a,b). This cyclotron incorporated a miniature Cs sputter negative ion source at the center of the cyclotron, injecting negatively-charged carbon ions at about $3 \mathrm{keV}$. Extraction energy was about $40 \mathrm{keV}$ at about a $10.5 \mathrm{~cm}$ radius. The cyclotron was operated at the 11th to 15 th harmonic to obtain the necessary resolution and relied solely on electrostatic focusing for ion confinement. It was found that such a device does indeed produce the necessary resolution for detection of ${ }^{14} \mathrm{C}$.

Unfortunately, ${ }^{14} \mathrm{C}$ could not actually be detected in the early version of the cyclotron because of the low output of the internal ion source which was used. It was necessary to rebuild the cyclotron to provide for a high-current external ion source. The cyclotron reported here is very similar in design to the earlier machine, with the addition of an external ion source and injection beamline, and is shown in Fig. 1.1.

Research on similar small cyclotrons as mass spectrometers has also begun in China (Chen et al., 1987, 1989b; Chen, Gao and Li, 1989a) and Yugoslavia (Subotić et al., 1990). These machines are both significantly more complex than the cyclotron discussed here. They show great promise, though they have not yet progressed as far as this work. 
Our initial work has emphasized ${ }^{14} \mathrm{C}$, partially because of the many users interested in this isotope, and partially because the required mass resolution and beam currents are relatively easy to obtain. Measurements of other radioisotopes, such as ${ }^{26} \mathrm{Al},{ }^{1 /} \mathrm{Be}$ and ${ }^{3}$ He are also possibilities with the cyclotrino but have not been pursued (Appendix A).

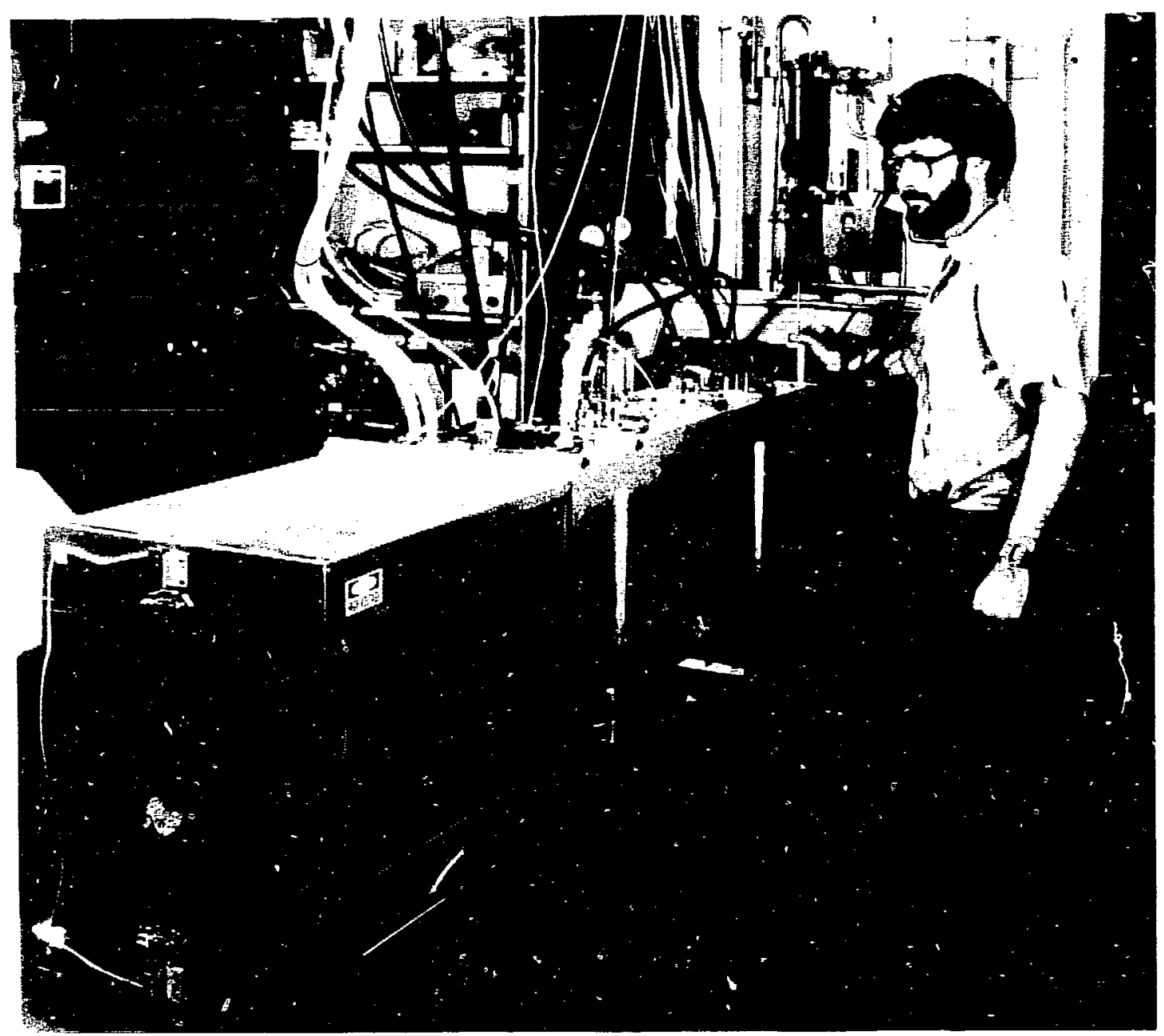

Figure 1.1 External view of the cyclotrino. (CBB 874-3258)

While the initial impetus for the small cyclotron was archaeological research, biomedical researchers have become interested in this tool as well. In studies of the human body there are limits on the amount of ${ }^{14} \mathrm{C}$ that can be safely given to a patient. For some metabolic studies it is necessary to separate blood into its various constituent fractions and to determine the amount of ${ }^{14} \mathrm{C}$ in each fraction, which may be so small as to contain less than $1 \mu \mathrm{g}$ of carbon. Decay counting does not yield the required sensitivity for many studies. Since an activity of $1 \mathrm{dpm}$ or so is needed to make a measurement in a reasonable 
amount of time, a $1 \mathrm{hg}$ sample must be enriched $7 \times 10^{4}$ times above modern levels to be measurable. A small cyclotron having a carbon current of $10 \mu \mathrm{A}$ and a detection efficiency of $0.1 \%$ would give about 4 counts per minute with modern carbon, improving the count rate by $51 / 2$ orders of magnitude! The cyclotrino would be very helpful in such metabolic studies. In fact, it may be feasible to connect the output of a liquid chromatography system to an appropriate ion source, injecting samples into the cyclotrino directly (Sargent, private communication). 


\section{CYCLOTRON DESIGN}

\subsection{General Design Considerations}

In a cyclotron, charged particles orbit freely in a magnetic field. The orbital frequency of these ions (the cyclotron frequency, $\omega=q B / m$ ) is a constant for nonrelativistic particles. The kinetic energy of a charged particle in a cyclotron may be increased by the imposition of electric fields which are driven synchronously at the cyclotron frequency or at a multiple of it. These electric fields establish a resonance condition for ions of the selected mass. Off-mass ions eventually fall out of synchronization with the alternating electric fields and are lost.

In a traditional two-dee cyclotron, each dee subtends $180^{\circ}$. Thus the electric fields are imposed at two positions in the particle's orbit, displaced $180^{\circ}$ from each other. In such a design the electric fields may be driven at he cyclotron frequency or at any odd harmonic of the cyclotron frequency. Such harmonic operation will improve the resolution of a cyclotron used as a mass spectrometer, since mass resolution is roughly proportional to the product of the number of turns and the harmonic (Welch, 1984a; Welch et al., 1987a). Typically, sinusoidal excitation is used, though with the relatively low RF voltages occurring in a cyclotron used as a low-energy mass spectrometer, non-sinusoidal excitation could be considered and has been suggested by other researchers (Chen er al., 1989b).

This two-dee design allows one dee to be fixed at ground potential while the other is driven with the alternating RF waveform. The grounded dee may be replaced by a narrow strip ("dummy dee"), freeing a large area for insertion of electrostatic deflection channels, dee probes, etc.

Design of the cyclotron itself is essentially the same as that described previously by our group (Welch, 1984a; Welch et al., 1984b, 1987a,b; Bertsche et al., 1987, 1988). A beam of carbon ions is introduced into the cyclotron. The cyclotron's magnetic field and RF frequency are tuned such that only the desired species $\left({ }^{14} \mathrm{C}\right)$ is accelerared; other species eventually fall out of phase with the RF and cease being accelerated, never reaching extraction radius. The cyclotrino operates with negative ions to avoid interference from ${ }^{14} \mathrm{~N}$. With ${ }^{14} \mathrm{~N}$ eliminated, the nearest interfering mass is the molecular ion ${ }^{13} \mathrm{CH}$, which is heavier than ${ }^{14} \mathrm{C}$ by a part in 1800 (Appendix A). This sets the minimum resolution requirements. The necessary resolution is obtained by operating at a high harmonic (1/th to 15 th) of the fundamental cyclotron frequency and by using a very flat magnetic field. 
A 12-inch NMR-type laboratory magnet is used, ope:ated at about 10kG. Beam is injected at an energy of $5 \mathrm{keV}$ with a $2.8 \mathrm{~cm}$ radius and extacted at about $40 \mathrm{keV}$ with a 10 $\mathrm{cm}$ radius. Ions must gain about $260 \mathrm{eV}$ in the first two gap crossings for about half of them to clear an electrostatic deflection channel in the center of the cyclotron (see section 2.2).

A computer program was written to simulate ion motion in the cyclotron. This program follows the approach of Cohen (1953) and Welch (1984a) in that the changes in energy, axial velocity and ion phase are calculated and assumed to occur instantaneously at each gap crossing. The program allows input of arbitrary ion characteristics (axial position, axial velocity and ion phase with respect to the RF waveform) and cyclotron characteristics (RF voltage, harmonic and frequency offset). An ion can be followed through the cyclotron to see the effects of different conditions.

\subsection{Energy Gain}

The energy gain of particles in a cyclotron is a result of the electric fields which exist across the dee gap. If this field distribution can be calculated, parameters such as energy gain per tum, tum separation, and number of turns can be calculated as well. The field distribution may be easily solved using certain approximations.

Firstly, we may make the electrostatic approximation. In this cyclotron, the time taken for the electric field to propagate in the gap region is so small as to be negligible, i.e. $b / c \ll 1 / \omega_{R F}$ where $b$ is the dee height, $c$ is the speed of light, and $\omega_{R F}$ is the RF frequency. Thus, the electric potential distribution between the dees is approximately that for an electrostatic problem; the solution is just the electrostatic potential with a timevarying amplitude.

Secondly, we may assume that the gap between the dees is infinitesimal. While this assumption is not necessary (solutions for the case of a finite gap have been presented by Murray and Ratner (1953) and Reiser [1971)), it simplifies the computations greatly and is 
a good approximation in the case of this cyclotron. The dees are not excited at extremely high voltages, so it has been possible to space them very close together. (The gap is 1 to 2 $\mathrm{mm}$, while the height is $8 \mathrm{~mm}$.) The assumption of an infinitesimal gap will only cause errors for particles traveling very close to the dees; the field near the median plane will be relatively unaffected by this approximation.

With these approximations, an analytical expression for the electric potential in the gap region may be obtained by conformal mapping:

$$
\Phi(y, z)=V\left[1-\frac{1}{\pi} \tan ^{-1}\left(\frac{\cos \frac{\pi z}{b}}{\sinh \frac{\pi y}{b}}\right)\right]
$$

The resulting electric fields are:

$$
\begin{aligned}
& E_{y}=-\frac{v}{b}\left(\frac{\cos \left(\frac{\pi z}{b}\right) \cosh \left(\frac{\pi y}{b}\right)}{\cos ^{2}\left(\frac{\pi z}{b}\right)+\sinh ^{2}\left(\frac{\pi y}{b}\right)}\right) \\
& E_{2}=-\frac{V}{b}\left(\frac{\sin \left(\frac{\pi z}{b}\right) \sinh \left(\frac{\pi y}{b}\right)}{\cos ^{2}\left(\frac{\pi z}{b}\right)+\sinh ^{2}\left(\frac{\pi y}{b}\right)}\right)
\end{aligned}
$$

where a sinusoidal excitation is assumed:

$$
\begin{aligned}
V & =V_{m} \cos (H \omega t+\phi) \\
& =V_{m} \cos \left(\frac{H y}{r}+\phi\right), \text { for } H y \ll r
\end{aligned}
$$

and where variables are defined as:

$y=$ distance from gap plane (in direction of ion motion)

$z=$ distance from median plane

$b=$ dee height (distance between top and bottom surfaces)

$V=$ instantaneous voltage across gap (dee voltage)

$V_{m}=$ peak dee voltage

$H=$ harmonic number $=\omega_{R F} / \omega$ 
$\omega=$ cyclotron frequency $=q B / m$

$\omega_{R F}=\mathrm{RF}$ oscillator frequency

$q=$ ion charge

$B=$ magnetic field strength

$m=$ ion mass

$E_{y}=$ electric field component in $y$ direction

$E_{2}=$ electric field component in $z$ direction

$\phi=\mathrm{RF}$ phase at which ion crosses dee gap

$r=$ cyclotron radius $=v / \omega$

The electric field is shown schematically in Figure 2.1.

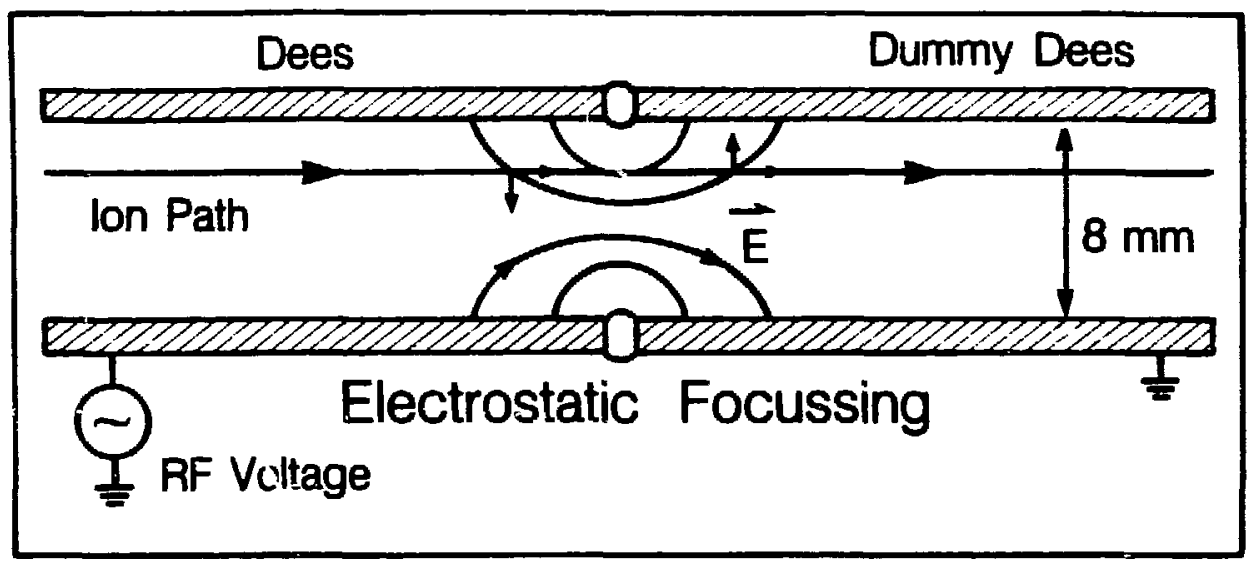

Figure 2.1 Electric field across dee gap. Electrostatic focusing will be discussed in section 2.3. (XBL 8410-8761, froni Welch, 1984a)

Knowing the field distribution in the gap region, the change in kinetic energy due to a gap crossing may be easily approximated:

$$
\delta T=\int F_{y} d y \equiv \int_{-\infty}^{\infty} q E_{y} d y
$$

where $T=$ ion energy and the integral extending to infinity assumes that $b<r$. This integral is difficult to solve in general, but if one assumes that $\delta T \ll T$ (a good approximation in our case), the cyclotron radius (which appears in trigonometric arguments in the expressions for electric field) may be assumed constant actoss the gap. The integral may be solved by writing the denominator as a difference of squares and expanding with partial fractions (Appendix B): 


$$
\delta T \cong-q V_{m} \cos \phi\left[\frac{\cosh \frac{H z}{r}}{\cosh \frac{H b}{2 r}}\right]
$$

The bracketed quantity is a transit-time factor (Livingood, 1961, \$7-10; Welch, 1984a; Welch et al., 1987a); it tends to reduce the energy gain for ions which traverse the gap slowly compared to the period of the sinusoidal excitation of the dees.

For a negatively-charged ion (as is used in this machine) energy gain is provided only for $\phi$ in the first or fourth quadrant. In the second and third quadrants ions will lose energy.

From (2.6) it is simple to calculate the change in radius per gap crossing:

$$
\begin{aligned}
\delta r & \cong \frac{r \delta T}{2 T} \\
& \cong \frac{-q V_{m} \cos \phi}{m r \omega^{2}}\left[\frac{\cosh \frac{H z}{r}}{\cosh \frac{H b}{2 r}}\right] \\
& \equiv \frac{r(\delta T)_{\max } \cos \phi}{2 T}\left[\frac{\cosh \frac{H z}{r}}{\cosh \frac{H b}{2 r}}\right]
\end{aligned}
$$

where $\delta T_{\max }=$ maximum possible energy gain per turn $=-q V_{m}$ (negative ions are assumed). Since there are two accelerating gaps per orbit, the radial separation of orbits is twice this quantity.

The increase in radius given by the irst two gap crossings must be sufficient for the ions to clear the inner deflector (see section 3.6) after the first orbit. The beam width at the position of this deflector, which is $90^{\circ}$ from the dee gap, is a minimum and is about $2 \mathrm{~mm}$. Thus, a $2 \mathrm{~mm}$ increase (corresponding to a $520 \mathrm{eV}$ increase for a $5000 \mathrm{eV}$ beam) will allow all ions to clear, while a $1 \mathrm{~mm}$ increase (corresponding to a $260 \mathrm{eV}$ increase) will allow about half to clear. We have found that about 300 to $400 \mathrm{~V}$ peak voltage is necessary to clear most of the ions when running at the 11 th to 15 th harmonic.

By imposing the additional assumptions that the phase $\phi$ is approximately constant throughout the acceleration process, and that ions remain near the median plane ( $z$ small), 
(2.7) may be integrated to find the approximate number of gap crossings for a given radius. The result is somewhat awkward to apply and is only approximate in any case. It is simpler and more useful to calculate the minimum number of turns by the very simple assumption that particles gain the maximum energy available at each gap crossing. Thus:

$$
\begin{aligned}
N_{\text {tums }} & \geq \frac{T_{f}-T_{0}}{2(\delta T)_{\max }} \\
& \geq \frac{T_{0}}{2(\delta T)_{\max }}\left(\frac{r_{f}^{2}}{r_{0}{ }^{2}}-1\right)
\end{aligned}
$$

where $T_{0}=$ initial ion energy, $T_{f}=$ extraction energy, $r_{0}=$ initial cyclotron radius and $r_{f}=$ cyclotron radius at extraction.

A more exact computation of the number of turns may be obtained with the computer program which was mentioned previously (section 2.1). For this cyclotron, operating at the 11 th harmonic with $300 \mathrm{~V}$ peak, one would expect at least 50 turns based on (2.8). The actual number of turns is shown in Fig. 2.2 as a function of input parameters. It is seen that ions entering with $0^{\circ}$ phase require 50 turns if they enter near the dees, since they gain the maximum energy at each gap crossing. They require more turns if they enter near the center of the dee gap because of the transit time effect. For ions which enter with $45^{\circ}$ phase, still more turns are required, the exact number depending on axial position and angle.

This cyclotron is typically operated with a harmonic $H$ of 11 to 15 and a peak voltage $V_{m}$ of 300 to 400 volts. The cyclotron frequency $\omega$ is about $1 \mathrm{MHz}$ with a magnetic field of about 1 Tesla, so the dees must be driven at 11 to $15 \mathrm{MHz}$ at 600 to 800 volts peak-to-peak. This is done with a modified amateur radio transmitter (Kenwood 930S) with an oven-controlled crystal driving a linear amplifier (ENI 3200L). The signal is matched to the capacitive load of the dees with a tunable matching network (Appendix $C$ ). A voltage monitoring circuit provides a measurement of the dee voltage (Appendix $C$ ). 


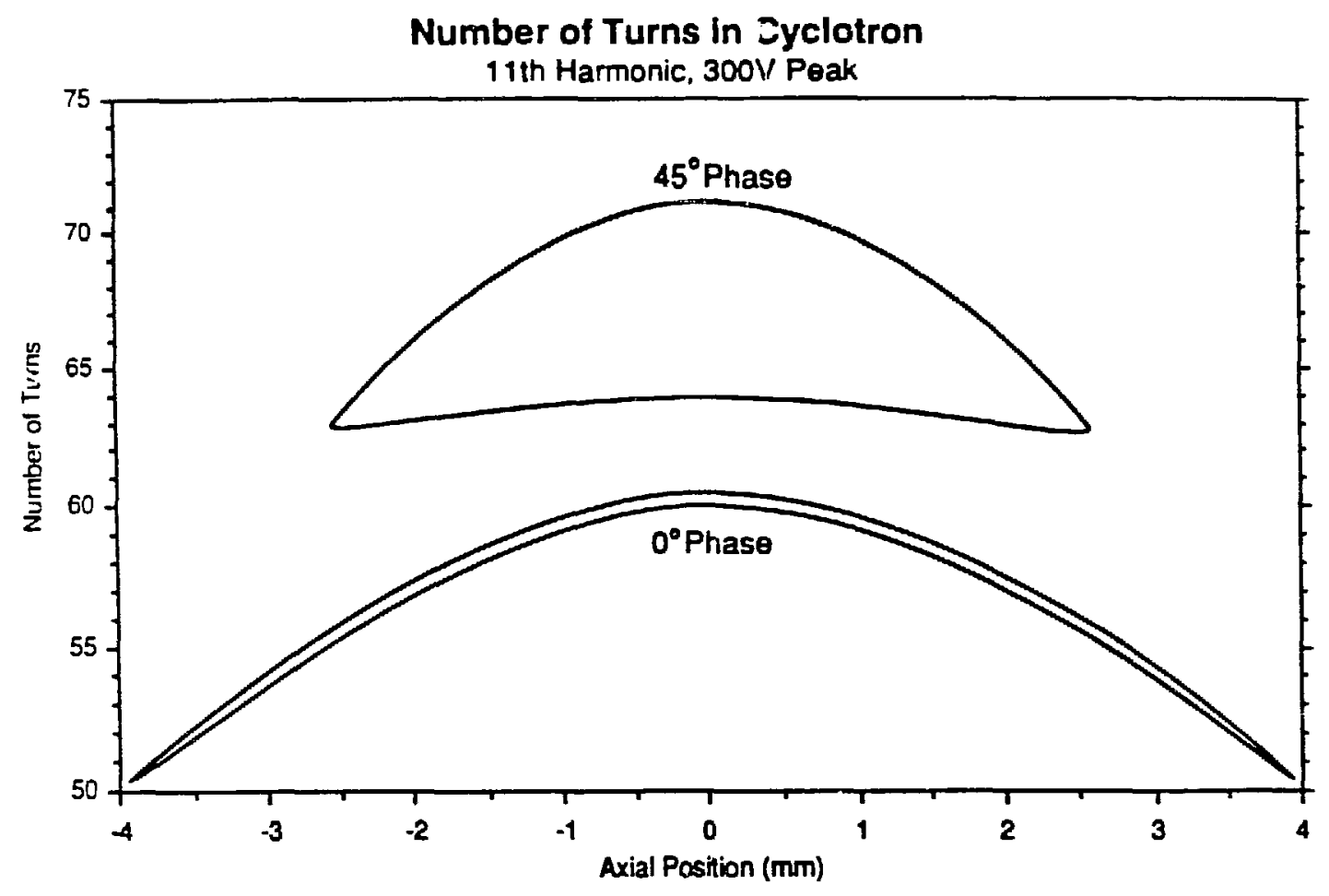

XBI $8911-4176$

Figure 2.2 Computer simulation of number of tums as a function of initial ion position, for ion phases of 0 and $45^{\circ}$. Initial ion angle (axial velocity) has been swept over the useful range. Operation is at the 11th harmonic with $300 \mathrm{~V}$ peak RF. (XBL 8911-4176)

\subsection{Stability}

In traditional cyclotrons, orbit stability is dependant primarily on the magnetic field index of the machine (Livingood, 1961, ch. 2). The magnetic field is given a radial gradient; the field index is defined as the ratio of the fractional change in $B$ to the fractional change in radius. In the cyclotrino it has been necessary to keep the field index as close to zero as possible to retain orbit isochronicity. Isochronous orbits are necessary if particles are to be retained for a large number of turns, which in tun is necessary for high resolution. Thus, orbit stability mechanisms in the cyclotrino are somewhat more subtle than in traditional designs. 


\subsubsection{Axial Stability}

In the absence of axial focusing, particles will eventually strike the inner surfaces of the dees and be lost. Some form of axial focusing must be provided to avoid this. Since the field index of the cyclotrino is zero, no focusing results from a magnetic field gradient. Sector focusing (azimuthal variations in magnetic field strength) could have been used to provide focusing while retaining orbit isochronicity. This is the focusing method used in most modern cyclotrons, but was judged more complex than necessary for this machine.

In the cyclotrino, axial stability is provided by electrostatic focusing. Electrostatic focusing is well understood (Cohen, 1953; Cohen and Rainwater, 1969) and has been recognized since the early days of the cyclotron (Rose, 1938; Wilson, 1938). Livingston, one of the members of Lawrence's original cyclotron team, has noted that "The electrostatic focusing between D's is one of the most important features leading to practical results, after the resonance principle itself." (Mann, 1953)

Electrostatic focusing arises from the fact that the electric field varies in both space and time. The axial impulse which an ion receives at a gap crossing may be approximated by:

$$
\begin{aligned}
\delta p_{z} & \cong \int_{t=-\infty}^{t=\infty} q E_{z} d t \\
& \cong q \int_{y=-\infty}^{y=\infty} \frac{E_{z}(y)}{V_{y}(y)} d y
\end{aligned}
$$

where, again, the approximation has been made that $b \ll r$. Again, it is beneficial to assume that $\delta T \ll T$. This simplifies trigonometric arguments as before, and also allows $v(y)$ to be approximated as constant and pulled out of the integral. The integral may be solved similarly to the integral for energy gain discussed above (Appendix B):

$$
\delta p_{z} \cong \frac{q V_{m} \sin \phi}{V}\left[\frac{\sinh \frac{H z}{r}}{\cosh \frac{H b}{2 r}}\right]
$$

For $\phi$ in the first or second quadrant a negative ion experiences a restoring force toward the median plane. These particles are focused axially, while those with $\phi$ in the third or fourth quadrant experience axial defocusing and are quickly lost. Thus only ions 
with $\phi$ in the first quadrant will experience simultaneous axial focusing and energy gain; only these ions will have a chance to be successfully exuracted from the cyclotron.

Equation (2.10) represents the axial momentum kick provided to an ion at each gap crossing. It is obviously nonlinear in $z$, but for small $z$ may be linearized:

$$
\delta p_{z} \cong \frac{q V_{m} H \sin \phi}{V r \cosh \frac{H b}{2 r}} z
$$

These discrete momentum changes may be approximated as a continuous momentum change, due to an equivalent force of:

$$
F_{\text {zequiv }}=\frac{\delta p_{2}}{\delta t}=\frac{\omega}{\pi} \delta p_{2}
$$

The equation of axial motion is:

$$
m \frac{d^{2} z}{d t^{2}}=F_{z \text { cquiv }}
$$

This differential equation may be solved for the frequency of axial oscillations, which is:

$$
\omega_{z}=\sqrt{\frac{-q V_{m} H \sin \phi}{\pi m r^{2} \cosh \frac{H b}{2 r}}}
$$

The axial betatron frequency for ions near the cyclotron median plane is defined as:

$$
v_{z} \equiv \frac{\omega_{z}}{\omega}=\sqrt{\frac{(\delta T)_{\max }}{T} \frac{H \sin \phi}{\pi \cosh \frac{H b}{2 r}}}
$$

In this cyclotron, ions start with $5 \mathrm{keV}$ energy and $3.8 \mathrm{~cm}$ cyclotron radius, exiting with about $35 \mathrm{keV}$ energy at a $10 \mathrm{~cm}$ radius. The dee height is $0.8 \mathrm{~cm}$. Operating at the 15 th harmonic with $400 \mathrm{~V}$ peak RF voltage, we find that the axial betatron frequency $v_{z}$ is $0.39 \sqrt{\sin \phi}$ initially and is $0.21 \sqrt{\sin \phi}$ at extraction. Thus, the potential resonance at $v_{z}=0.50$ due to mechanical imperfections is avoided (Livingood, 1961, ch 5); other potential resonances at $0.33,0.25$ and 0.20 are either mild enough or are traversed so 
rapidly that they do not cause problems. There are no coupled resonances to radial or phase motion in this machine since there are no radial or phase oscillations.

The frequency of these small axial oscillations will not remain constant throughout the acceleration process since $T$ and $r$ will change. Hence the amplitude of axial oscillations will no: remain constant, either. The WKB approximation may be used to determine the approximate variation in amplitude of these oscillations. Following the analysis of Livingood (1961, §2-9), one finds that the amplitude is proportional to $v_{2}{ }^{-1 / 2}$. For a given ion, then, the amplitude of axial oscillations is proportional to:

$$
\sqrt[4]{\frac{r^{2} \cosh \frac{H b}{2 r}}{\sin \phi}}
$$

Trajectories of ions may be studied with the computer simulation mentioned in section 2.1. The trajectory of a typical ion is shown in Fig. 2.3, illustrating axial oscillations and amplitude variations. 


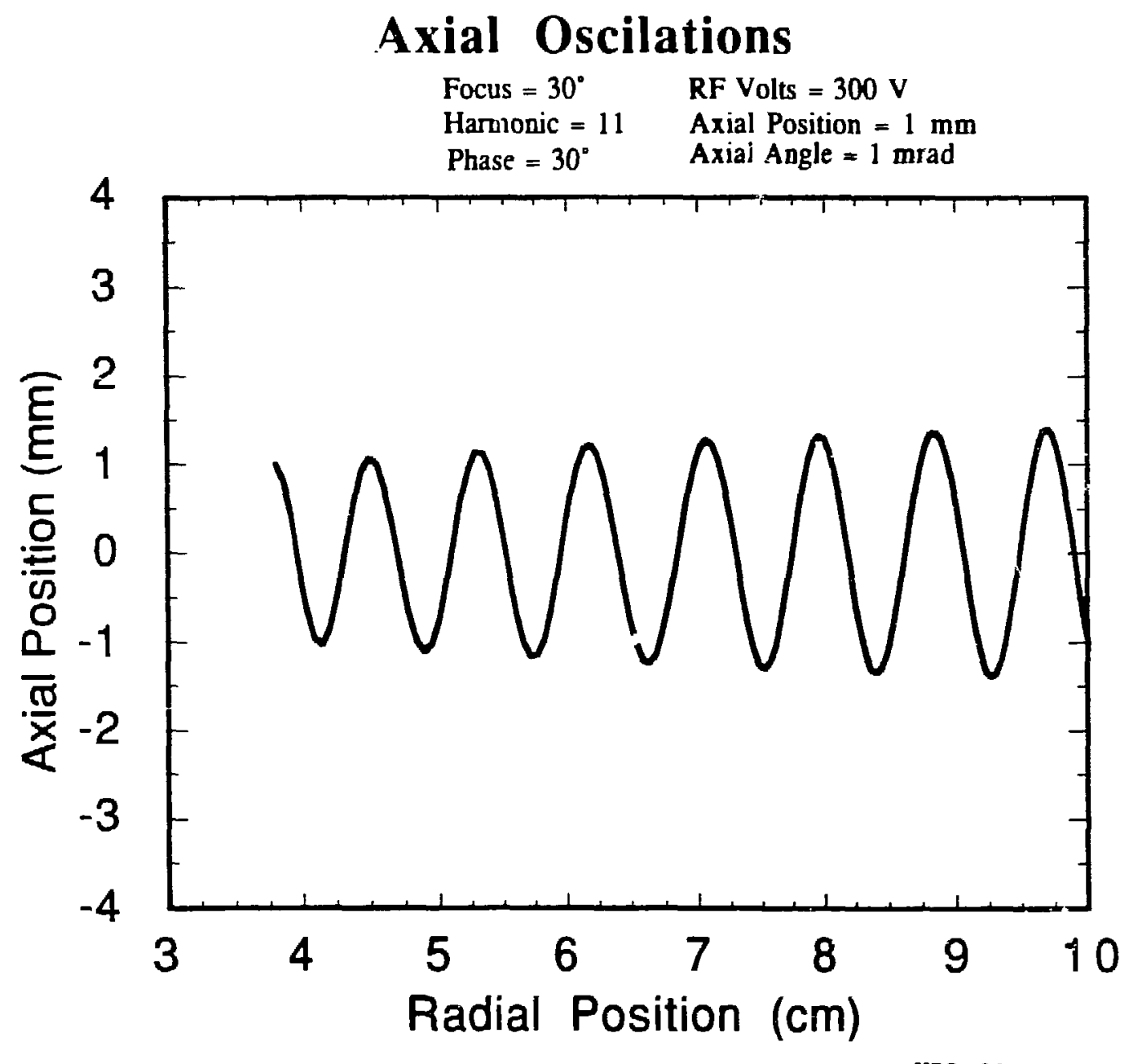

Figure 2.3 Simulated axial position of a typical ion as it traverses the cyclotron, showing axial oscillations. Operation is at the 11 th harmonic with $300 \mathrm{~V}$ peak RF. (XBL 8911-4220)

\subsubsection{Radial Stability}

There will be no focusing in the radial plane (the orbit plane) in this machine, provided that the B-field is uniform. But there will be perturbations to the radial motion. This motion can be analyzed conveniently by describing the location of the center of an ion's orbit (Milinkovic, Subotic and Fabrici, 1988). When not in the dee gap, the orbit center is fixed and the ion describes circular motion about this point with a radius determined by ion energy. When traversing a dee gap, an ion gains energy and the center of its orbit shifts. 
As an ion gains energy, the center of its orbit moves in alternate directions at each gap crossing, appearing to hop back and forth along the dee gap. The distance of each hop decreases in magnitude as the ion approaches extraction radius. For an ion whose center lies on the dee gap, the magnitude of each of these hops may be expressed as:

$$
\delta x=\frac{r}{\sqrt{T_{0}}} \delta(\sqrt{T}) \approx \frac{r_{0}^{2}}{2 T_{0}} \frac{\delta T}{r}
$$

The distances of these hops may be summed to find the net offset of orbit centers at extraction radius due to the acceleration process. For $\delta T \ll T_{0} \ll T_{f}$ this sum converges to a total shift of half of the initial hop:

$$
\Delta x_{\mathrm{acc}} \approx \frac{1}{2}(\delta x)_{0}
$$

It is important to examine the effect of perturbing the initial orbit center position from its assumed initial position on the dee gap. There are two dimensions to be considered. If the perturbation is along the dee gap by an amount $\Delta x_{0}$, all of the succeeding orbit centers will be offset the same amount and the final position will simply be perturbed by a distance $\Delta x=\Delta x_{0}$.

However, if the perturbation is orthogonal to this, by a distance $\Delta y_{0}$ away from the dee gap, there is a coupling to other dimensions. The ion will reach the gap at a different time, hence at a different RF phase:

$$
\Delta \phi=\frac{H \Delta y_{0}}{r_{0}}
$$

If $y$ is positive as shown in Fig. 2.4, the phase shift is also positive and the ion arrives later. At the next gap the ion will arrive early by the same amount. Since the energy gained at each gap crossing depends on phase, the energy gains will be unbalanced and the hops along the dee gap will be unequal. The ion's orbit center will drift in the $x$ dimension while it maintains a constant offset $\Delta y=\Delta y_{0}$ from the dee gap.

The total offset $\Delta x_{\text {off }}$ due to an offset $\Delta y_{0}$ from the dee gap may be easily approximated for a simplified case. If it is assumed that $\Delta y_{0}<<r / H, \phi$ constant (no phase bunching-discussed in the next section), and no transit time effect, the $\delta x$ shifts experienced at each gap crossing may be summed to find: 


$$
\Delta x_{\text {off }} \approx \frac{H \Delta y_{0}}{4} \tan \phi \ln \frac{T_{f}}{T_{0}}
$$

Thus the ions will drift in the $x$ dimension as shown in Fig. 2.4. For typical operating parameters of $T_{0}=5 \mathrm{keV}, T_{f}=35 \mathrm{keV}, H=15$, and assuming that $\phi \approx 30^{\circ}$, one finds the drift $\Delta x_{\text {off }}=4 \Delta y_{0}$.

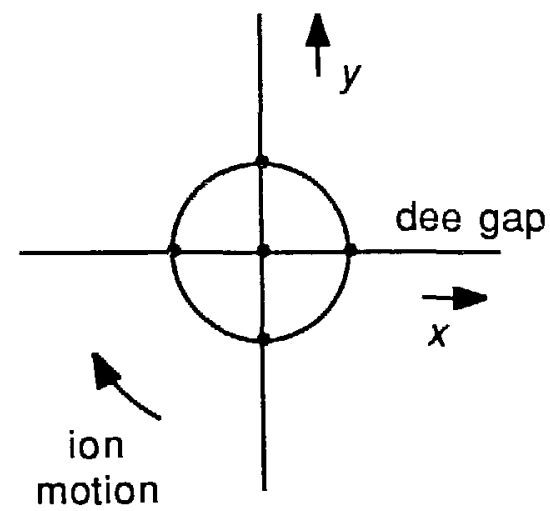

(a)

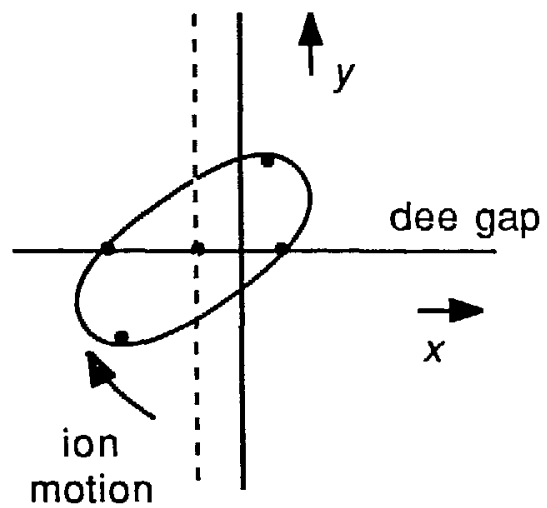

b)

Figure 2.4 Loci of orbit centers, showing radial perturbations to ion trajectories. Initial distribution of orbit centers is shown in $(a)$, leading to the final distribution in $(b)$. The distribution is shifted according to (2.16) and is distorted according to (2.17). (XBL 8911-4172)

\subsubsection{Phase Stability}

In a simple cyclotron with no sector focusing, phase stability is a function of field index. With a field index of zero, as in this design, one would expect no phase focusing. Phase focusing relies on a change in the time required to make one orbit. If orbits are truly isochronous, there can be no phase focusing and will be a condition of neutral phase stability. One would think that a flat field cyclotron such as this would be truly isochronous and would thus have no coupling of orbit time to phase of gap crossing, resulting in neutral phase stability for all ion phases. Such is not the case. It is true that there are no phase oscillations in this machine, but there is a phase bunching effect.

Although a flat-field machine has no change in orbit time as a function of radius, there is nevertheless a coup.'ing of orbit time to phase of gap crossing as noted by Chen and collaborators (Chen et al., 1987; Chen, Gao and $\mathrm{Li}, 1989 \mathrm{a}$ ). In this sense a flat-field 
cyclotron is not wu, isochronous. A flat field implies that the cyclotron frequency is constant, but it does not follow that the orbit time is a constant as well. Constant cyclotron frequency means that a fixed angle is subtended per unit time. Interestingly, the angle subtended in crossing the dee gap is not a constant; it depends on the details of energy gain and is a function of the phase of the particle.

The region of energy gain is not infinitesimally thin but is extended in space due to fringing of the electric field in the gap. To see how this gives rise to phase shifts, one may imagine that the energy gain occurs at two planes physically separated from the dee gap by a fixed distance. It can be seen by graphical construction (Fig. 2.5) that if more energy gain occurs at the second plane the total subtended angle in traversing a half-orbit is greater than $180^{\circ}$, more time is taken and the particle phase is retarded. If more energy gain occurs on entering the dee gap, the total subtended angle for a half-orbit is less than $180^{\circ}$ and particle phase is advanced.
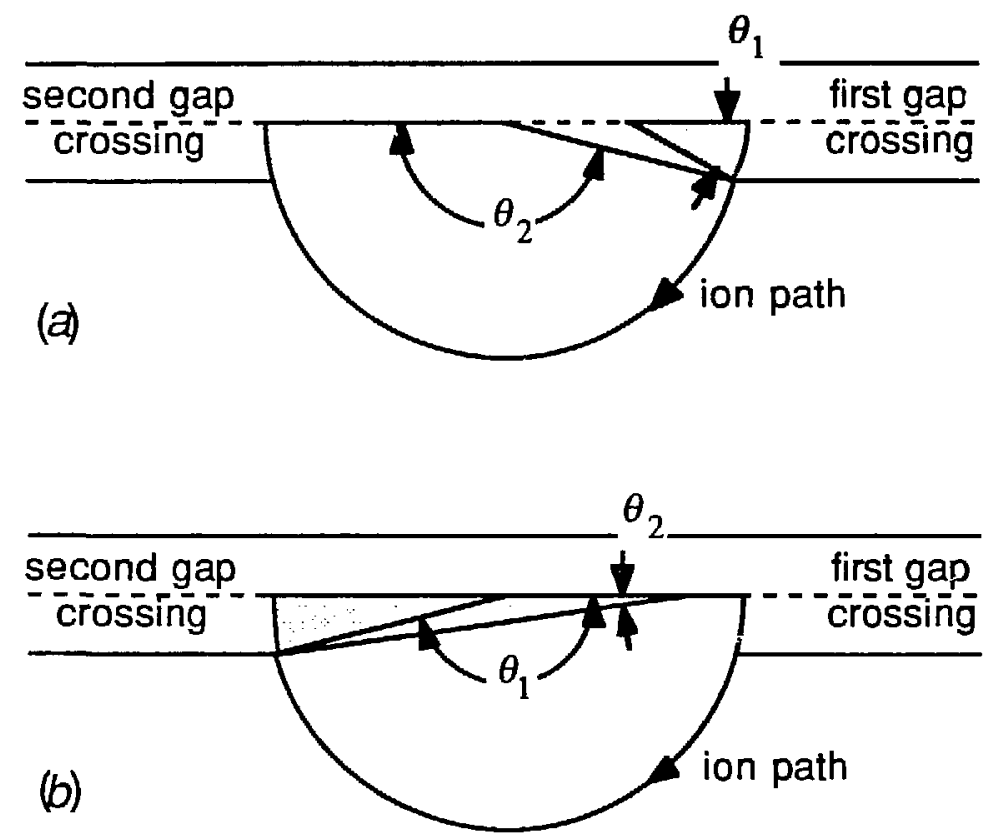

Figure 2.5 Origin of phase bunching in the small cyclotron. In $(a)$, ions experience more energy gain upon exiting dee gap than upon entering; total angle $\left(\theta_{1}+\theta_{2}\right)$ subtended in a half-orbit is greater than $180^{\circ}$ and ion phase is retarded. In (b), more energy is gained upon entering the dee gap; total subtended angle is less than $180^{\circ}$ and ion phase is advanced. (XBL 8911-4173)

Consider operation in the first quadrant of the RF cosine wave, which is necessary for simultaneous energy gain and axial focusing. A particle arriving at the waveform peak 
receives nearly equal energy gains on both sides of the dee gap, thus no phase shift. A particle arriving later gets more energy gain on entering the gap region than on exiting since the cosine waveform have decreased in magnitude by the time the ion exits, thus advancing its phase. It is seen that particle phase tends to be bunched toward the peak of the RF waveform.

In a cyclotron, the energy gain does not only occur at two planes, but is distributed across the gap with a distribution which depends on a particle's axial position and velocity. The phase change due to a single gap crossing may be calculated by integrating the angle subtended as an ion crosses the gap region and subtracting the total angle which would have been subtended if the energy gain had all occurred at an infinitesimal-width gap. Thus:

$$
\delta \phi_{\text {ion }} \cong \int_{\text {gap }} \frac{d y}{\gamma}-\left(\frac{1}{r_{0}} \int_{\text {first half gap }} d y+\frac{1}{r_{j}} \int_{\text {second half gap }} d y\right)
$$

Assuming that $\delta T \ll T$, and that $b \ll r$ (the distance over which the electric field extends is on the order of $b$ ), the phase change experienced in a single gap crossing may be reduced to (Appendix B):

$$
\begin{aligned}
\delta \phi & \equiv \frac{H}{2 r T} \int_{y=-\infty}^{y=\infty} y d T \equiv \cdot \frac{H}{2 r T} \int_{-\infty}^{\infty} y F_{y}(y) d y \\
& \cong \frac{q H}{2 r T} \int_{-\infty}^{\infty} y E_{y}(y) d y
\end{aligned}
$$

where $\phi_{\text {ion }}=$ ion phase and $\phi=\mathrm{RF}$ phase $=(H)$ (ion phase).

This phase shift has been expressed in terms of RF phase $(\phi)$, which is greater than ion phase $\left(\phi_{i o n}\right)$ by a factor of $H$. This allows for operation at a harmonic of the cyclotron frequency. The integral may be solved in a similar manner to the previous integrals for energy gain and axial momentum gain (Appendix B):

$$
\delta \phi \equiv \frac{q V_{m} H b}{4 r T} \sin \phi\left(\frac{\cosh \left(\frac{H z}{r}\right) \sinh \left(\frac{H b}{2 r}\right)+\frac{2 z}{b} \sinh \left(\frac{H z}{r}\right) \cosh \left(\frac{H b}{2 r}\right)}{\cosh ^{2}\left(\frac{H b}{2 r}\right)}\right)
$$


In deriving this expression it has been assumed that $\delta T \ll T$ and $b \ll r$, and that the gap between dees is infinitesimal. The total phase shift of an ion at extraction radius may be estimated with some additional approximations. If it is assumed that the ions remain near the median plane ( $z$ small), (2.20) may be approximated as:

$$
\begin{aligned}
\delta \phi & \cong \frac{q V_{m} H b}{4 r T} \sin \phi\left(\frac{\sinh \left(\frac{H b}{2 r}\right)}{\cosh ^{2}\left(\frac{H b}{2 r}\right)}\right) \\
& \equiv \frac{-\delta T_{\max }}{T} \frac{H b}{4 r} \sin \phi\left(\frac{\sinh \left(\frac{H b}{2 r}\right)}{\cosh ^{2}\left(\frac{H b}{2 r}\right)}\right)
\end{aligned}
$$

If the additional assumptions are made that the total phase shift is small ( $\phi \equiv$ constant) and that many turns are made in the cyclotron, the phase change $\Delta \phi_{\mathrm{cyc}}$ as an ion traverses the cyclotron, which is the summation of the individual phase shifts $\delta \phi_{\mathrm{RF}}$ at each gap crossing, may be approximated by an integral, which may then be simplified using (2.7) and (2.21):

$$
\begin{aligned}
\Delta \phi_{\mathrm{cyc}} & \cong \int \delta \phi=\int \frac{\delta \phi}{\delta r} d r \cong-\frac{H b}{2} \tan (\phi) \int_{r_{0}}^{r_{f}} \frac{1}{\rho^{2}} \tanh \left(\frac{H b}{2 \rho}\right) d \rho \\
& \cong-\tan (\phi) \ln \left(\frac{\cosh \left(\frac{H b}{2 r_{0}}\right)}{\cosh \left(\frac{H b}{2 r_{f}}\right)}\right)
\end{aligned}
$$

It is apparent that the phase $\phi$ will not be constant as an ion traverses the cyclotron. This phase shifting may be partially compensated by offsetting the RF frequency slightly, giving an additional, constant phase shift at each gap crossing.

Suppose the RF frequency is offset slightly so that an ion of the desired species, making its first gap crossing at RF phase $\phi_{s}$, also makes its final gap crossing at phase $\phi_{s}$. Then:

$$
\Delta \phi_{\mathrm{RF}}=-\Delta \phi_{\mathrm{cyc}}
$$


where:

$$
\Delta \phi_{\mathrm{RF}} \propto N_{c} \propto \frac{1}{\delta r} \propto \frac{1}{\cos (\phi)}
$$

and:

$$
\Delta \phi_{\text {cyc }} \propto \tan (\phi)
$$

Consider an ion beginning with phase $\phi_{S}+\Delta \phi_{\text {in }}$. Its final phase will be offset from $\phi_{s}$ by an amount $\Delta \phi_{\text {out }}$. In general, $\Delta \phi_{\text {out }}$ will differ from $\Delta \phi_{\text {in }}$, implying a spreading or bunching of ion phases. A phase compression factor $\alpha_{\text {phase }}$ can be defined:

$$
\begin{aligned}
\alpha_{\text {phase }} & \equiv \frac{\Delta \phi_{\text {out }}}{\Delta \phi_{\text {in }}} \\
& =\frac{d}{d \phi}\left(\phi_{s}+\Delta \phi_{\text {in }}+\Delta \phi_{\mathrm{RF}}+\Delta \phi_{\text {cyc }}\right) \\
& =1-\ln \left(\frac{\cosh \left(\frac{H b}{2 r_{0}}\right)}{\cosh \left(\frac{H b}{2 r_{f}}\right)}\right)
\end{aligned}
$$

Computer calculations of phase as a function of radius for paraxial ions are shown in Figs 2.6a-d. In Fig. 2.6a, the phase bunching for the 15th harmonic and $400 \mathrm{~V}$ peak RF voltage is seen. The effect of offsetting the RF frequency slightly is shown in Fig. 2.6b. Performance at the $31 \mathrm{st}$ harmonic is shown in Fig. 2.6c.d. It is obvious that the phase bunching increases as the harmonic is raised.

The approximation given above (2.23) is in good agreement with computer calculations for low $H$ (below about 9 ) in the cyclotrino. For $H=15$, the agreement with computer calculations is poor (Fig. 2.6b), while for $H=31$ the agreement is very poor (Fig. 2.6d), with $\alpha_{\text {phase }}$ becoming negative as calculated from (2.23), which is physically unreasonable. These disagreements seem to be because of the approximation $\phi \equiv$ constant, used in deriving (2.23). Negative values may be avoided by approximating $\alpha_{\text {phase }}$ as:

$$
\alpha_{\text {phase }} \cong \frac{1}{1+\ln \left(\cosh \left(\frac{H b}{2 r_{0}}\right) / \cosh \left(\frac{H b}{2 r_{f}}\right)\right)}
$$


which has the same asymptotic behavior as (2.23) for low $H$, but which cannot become negative as $H$ increases. This gives good agreement with computer simulations for $H=15$ in the cyclotrino, but is in error by about a factor of two at $H=31$.

The phase grouping effect tends to reduce the resolution of the cyclotron, as noted by Chen, Gao and $\mathrm{Li}$ (1989a). In fact, since the phase compression factor $(2.23,24)$ is independent of RF voltage (in the approximation that $\phi \equiv$ constant) and since the compression increases as the harmonic is increased (as seen in $(2.23,24)$ and Fig. 2.6), one may think that the resolution cannot be significantly increased by increasing the harmonic or lowering the RF voltage. This is not the case, however. Increasing the harmonic cr reducing the voltage will increase the number of turns an ion makes in the cyclotron; thus the average phase shift per turn tends to be reduced and the mass resolution tends to be increased.

The mass resolution may be approximated by computer simulations of an off-mass ion which enters on-axis at $0^{\circ}$ phase (a lower limit on the ion phases which can be focused), with the ion's mass adjusted so that it drifts in phase to exit at $60^{\circ}$ phase (an approximate upper limit on ion phases which can successfully exit the extraction channel). This gives rise to the family of curves shown in Fig. 2.7 for this cyclotron. It is obvious that the resolution is approximately proportional to $H / V$. In fact, as the harmonic is increased, resolution increases faster than does the harmonic, especially for high harmonics. (Doubling the harmonic from 15 th to 31 st triples the resolution).

It would appear from (2.7) that operation at 15 th harmonic and $400 \mathrm{~V}$ peak is not quite adequate for detection of ${ }^{14} \mathrm{C}$, with a resolution of about 1600 . However, we have found that it gives more than enough resolution to separate ${ }^{14} \mathrm{C}$ from ${ }^{13} \mathrm{CH}$. The approximate curves in (2.7) understate the actual resolution because a perfectly uniform magnetic field has been assumed in the analysis. In practice, the field strength drops slightly at larger radii, causing additional phase retardation. This tends to ircrease the resolution and reduce the transmission beyond what would be expected with a perfectly flat field. 


\section{Phase Bunching on Median Plane}

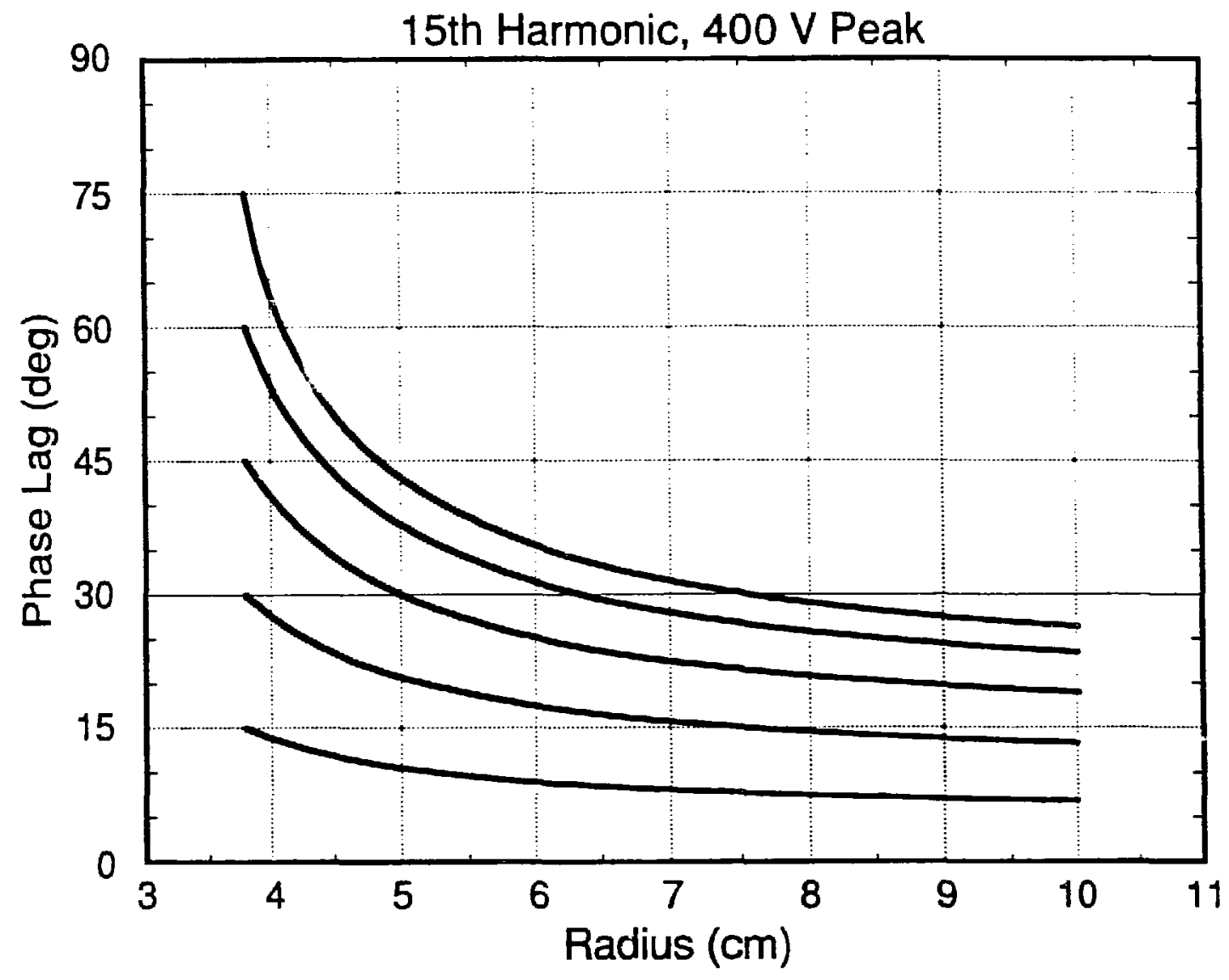

XBL $8911-4208$

Figure 2.6a Phase bunching for paraxial ions, with cyclotron cperating at the 15 th hamonic and $400 \mathrm{~V}$ peak. (XBL 8911-4208) 


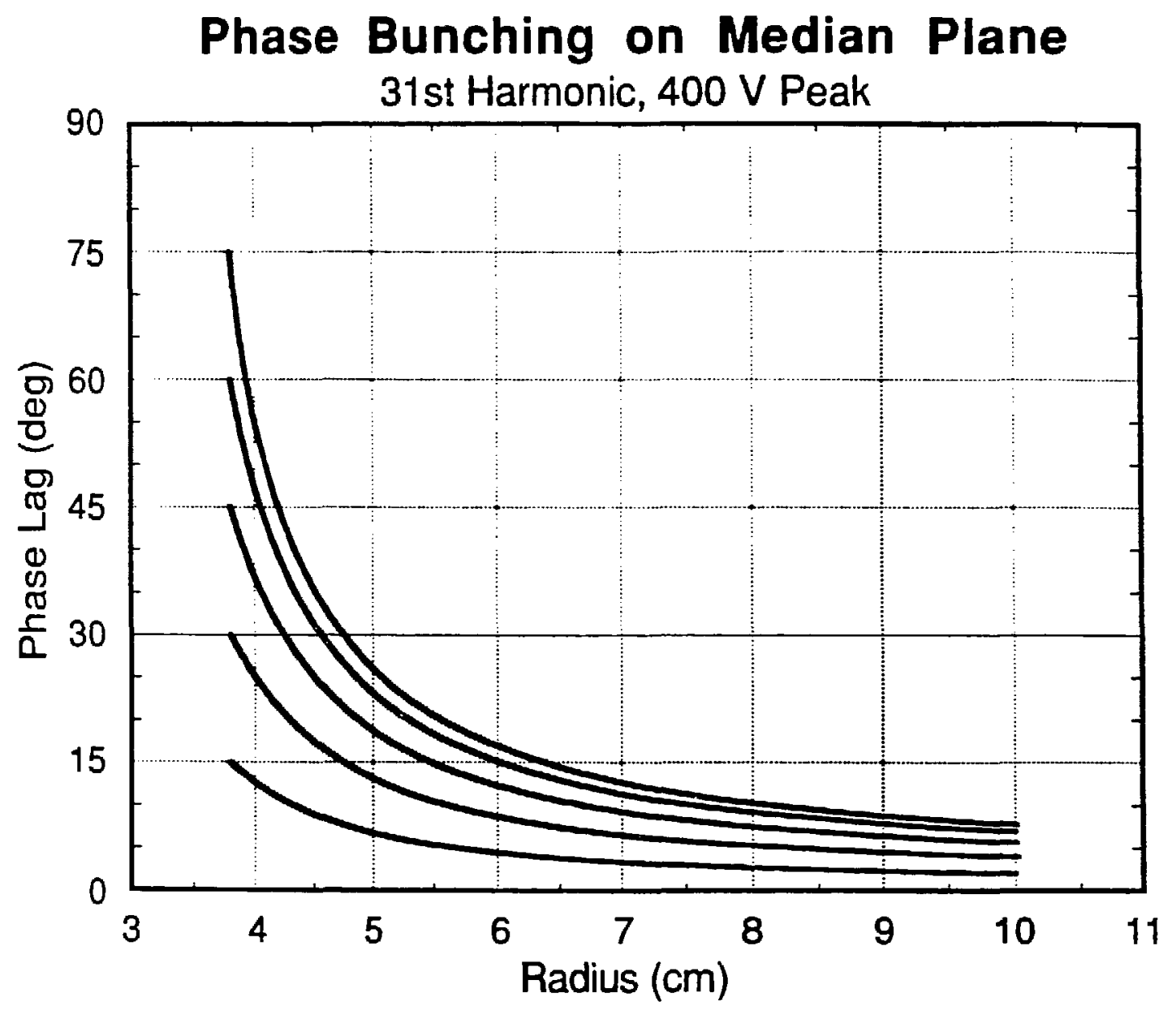

XBL B911-4212

Figure 2.6b Phase bunching for paraxial ions, with cyclotron operating at the 31 st harmonic and $400 \mathrm{~V}$ peak. (XBL 8911-4212) 


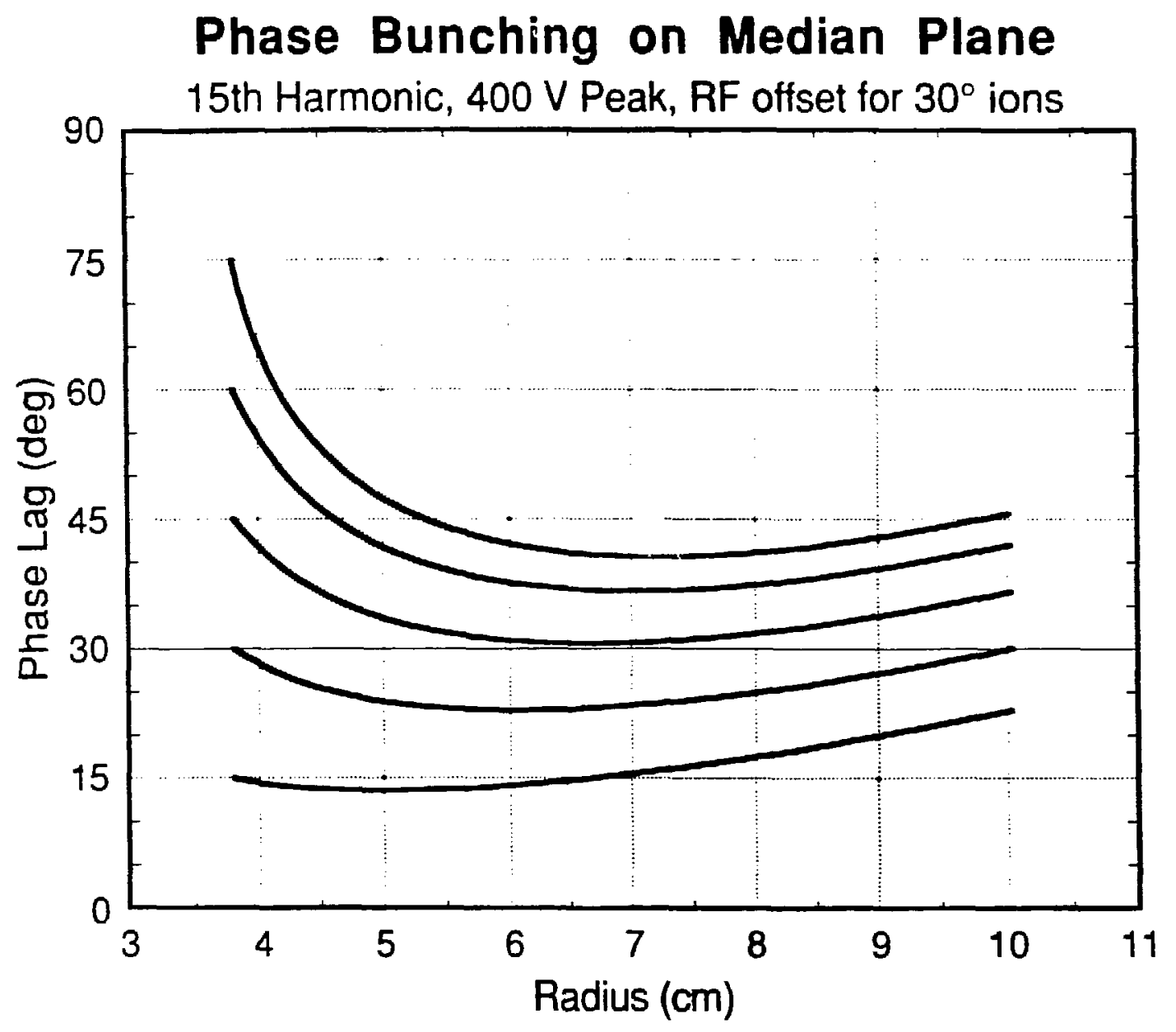

XBL $8911-4209$

Figure 2.6c Phase bunching for paraxial ions, with cyclotron operating at the 15 th harmonic and $400 \mathrm{~V}$ peak, with RF frequency offset slightly so that ions entering with $30^{\circ}$ phase also exit with $30^{\circ}$ phase. (XBL 8911-4209) 


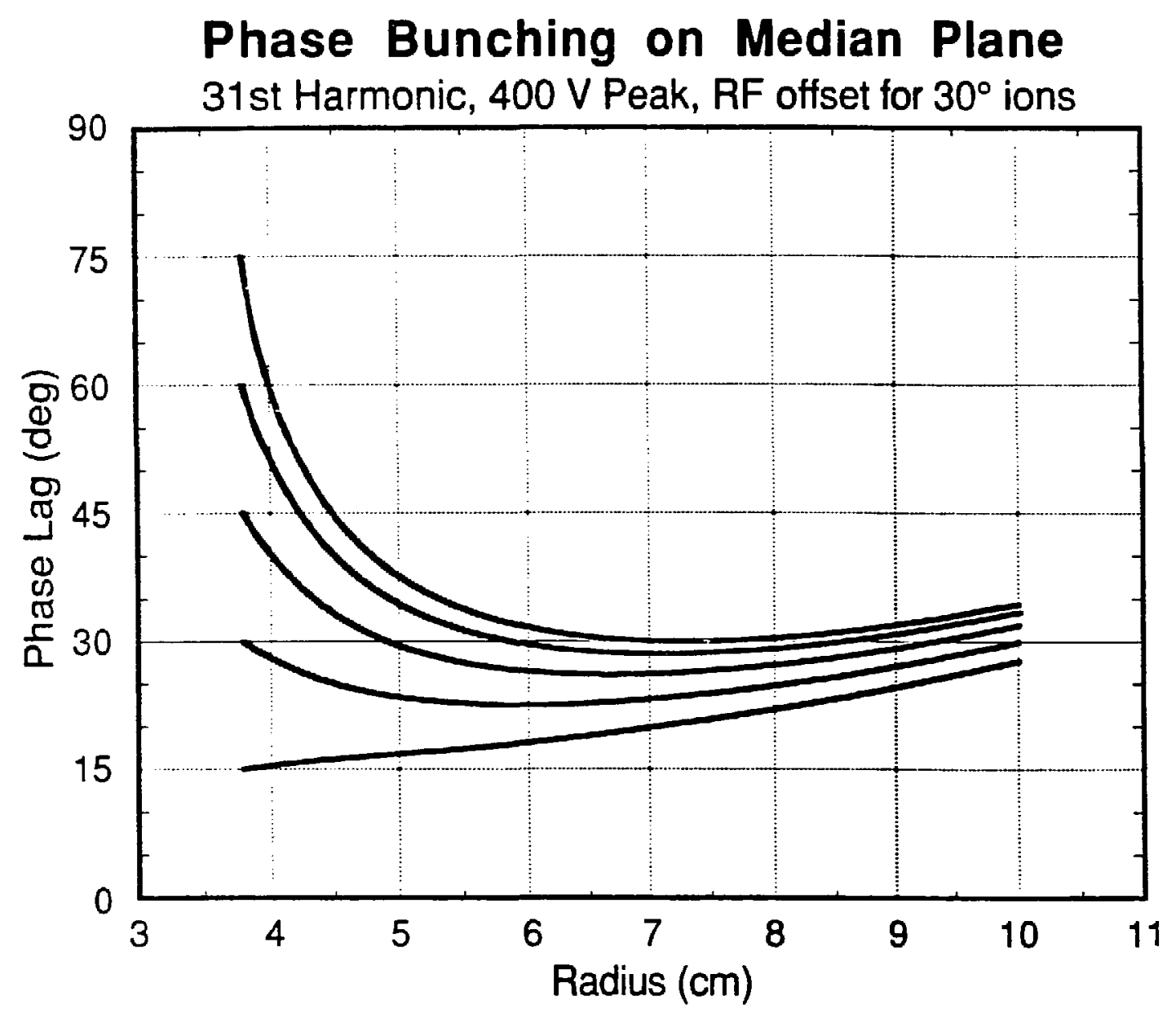

XBL $8911-4213$

Figure 2.6d Phase bunching for paraxial ions, with cyclotron operating at the 31 st harmonic and $400 \mathrm{~V}$ peak, with RF frequency offset slightly so that ions entering with $30^{\circ}$ phase also exit with $30^{\circ}$ phase. (XBL 8911-4213) 


\section{Approximate Resolution of Cyclotron}

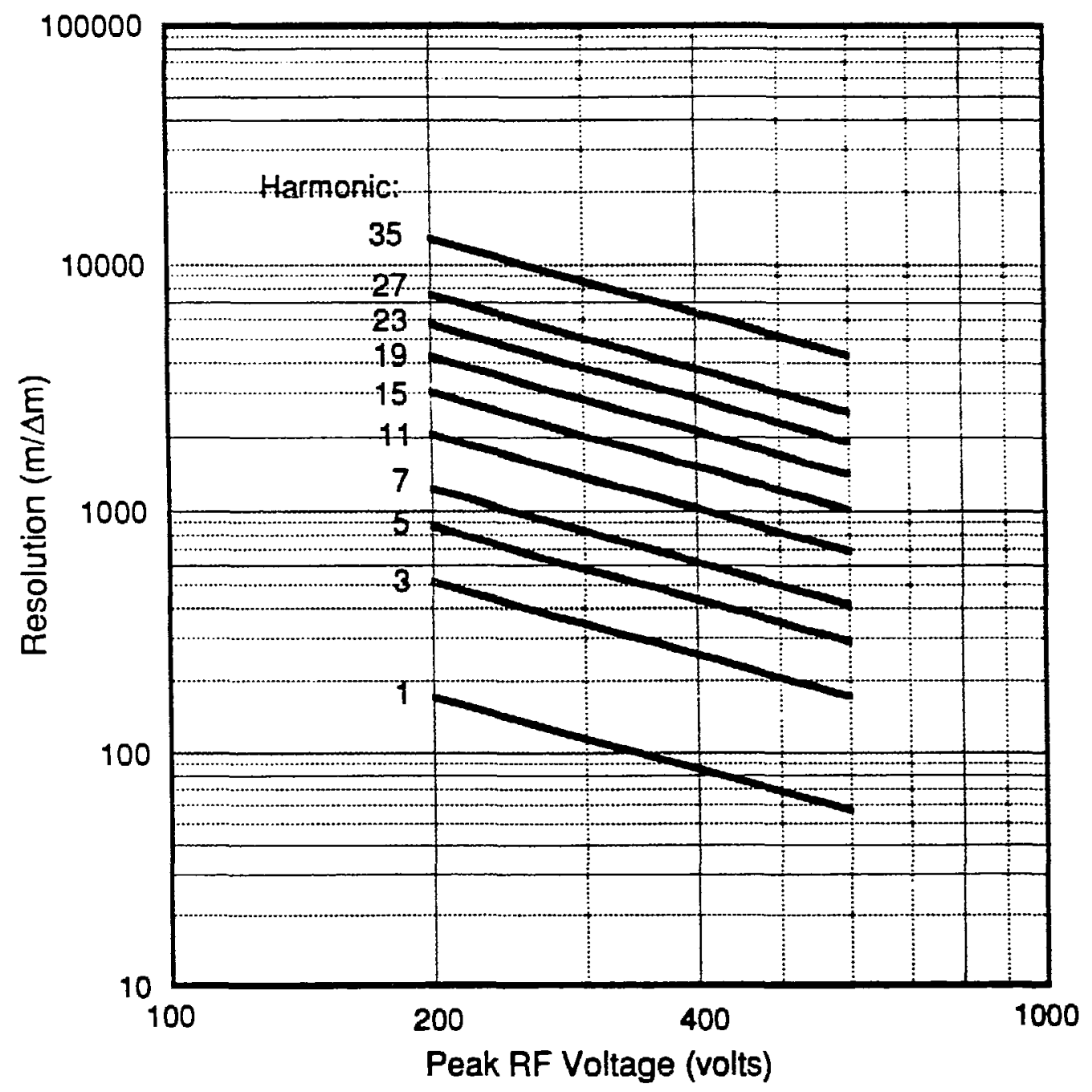

Figure 2.7 Approximate resolution of cyclotron in the presence of phase bunching. (XBL 8911-4263)

\subsection{Acceptance}

The acceptance of the cyclotron ia description of the characteristics required of a resonant ion for successful acceleration) can be described in terms of a 5-dimensional phase space. The components are axial position, axial velocity (or angle), radial position, radial velocity (or angle), and ion phase with respect to the RF waveform. 
The cyclotron acceptance must be matched to the ion source emittance to avoid losing beam. Extrapolating from estimates of the source emittance at $20 \mathrm{keV}$, it was estimated that about $80 \%$ of the source output should fall within $40 \pi \mathrm{mm}$-mrad at $5 \mathrm{keV}$. This estimate of $40 \pi \mathrm{mm}$-mrad was used for most emittance and acceptance calculations.

Radial acceptance properties may be calculated from the equations for radial motion (section 2.3.2). It is desired to minimize the spread of orbit centers at extraction radius. For a fixed emittance, the minimum spread of orbit centers along the dee gap may be obtained by allowing the orbit centers to "walk" a distance $\Delta x_{\text {off }}(2.17)$ about equal to the initial spread in centers, $\Delta x_{0}$. Thus, at $H=15$, the orbit centers would preferably lie in an ellipse with $\Delta x_{0}=4 \Delta y_{0}$. Since the harmonic is variable and will not necessarily be 15 th, a 6:1 ritio was decided upon. The focusing elements were designed to place orbit centers in an elliptical region along the dee gap with a semi-major axis of $3 \mathrm{~mm}$ and a semi-minor axis of $0.5 \mathrm{~mm}$. With an injection radius of $3.8 \mathrm{~cm}$, this encloses the desired emittance area of $40 \pi$ mmi-mrad.

Axial acceptance was determined with the computer simulation of cyclotron performance mentioned earlier. Since axial properties are coupled to ion phase, the results may be expressed as a family of axial phase plots, parametrized by ion phase (Fig. 2.8a-d). A beam of $40 \pi \mathrm{mm}$-mrad may be focused to a phase space ellipse at the dee gap with semiaxes of $2 \mathrm{~mm}$ and $20 \mathrm{mrad}$, matching the cyclotron's axial acceptance almost exactly.

In Fig. 2.8a, the axial acceptance for 11 th harmonic and $300 \mathrm{~V}$ peak is shown. Offsetting the frequency slightly, so that ions which enter with a $30^{\circ}$ phase lag also exit with a $30^{\circ}$ phase lag, improves the acceptance slightly (Fig. 2.8b). When losses due to first turn clearance (assuming $1 \mathrm{~mm}$ clearance, which is necessary for about half of the ions to clear) are included (Fig. 2.8c), it is seen that many ions are lost when operating with a $300 \mathrm{~V}$ peak RF voltage. Thus, most of our data was taken with $400 \mathrm{~V}$ peak and 15 th harmonic (Fig 2.8d). This results in about the same resolution as $300 \mathrm{~V}$ peak and 11 th harmonic, but gives lower loss due to the higher voltage. It was found that increasing the RF voltage from 300 to 400 volts approximately doubled the transmission through the cyclotron.

These axial acceptance plots are in general agreement with those published earlier (Welch, 1984a; Welch et al., 1987a). However, these simulations indicate that the acceptance for this new system is not quite so symmetric and encloses a somewhat smaller 
area. This is probably due to a higher ion injection energy and to the inclusion of phase bunching in the computer simulations.

Useful phase acceptance is estimated to be about $45^{\circ}$, based on Fig. $2.8 \mathrm{a}-\mathrm{d}$. If ion phase is less than about $15^{\circ}$, the axial acceptance shrinks dramatically due to insufficient axial focusing. If ion phase exceeds about $60^{\circ}$, not enough energy is gained to clear the inner deflector on the first turn.

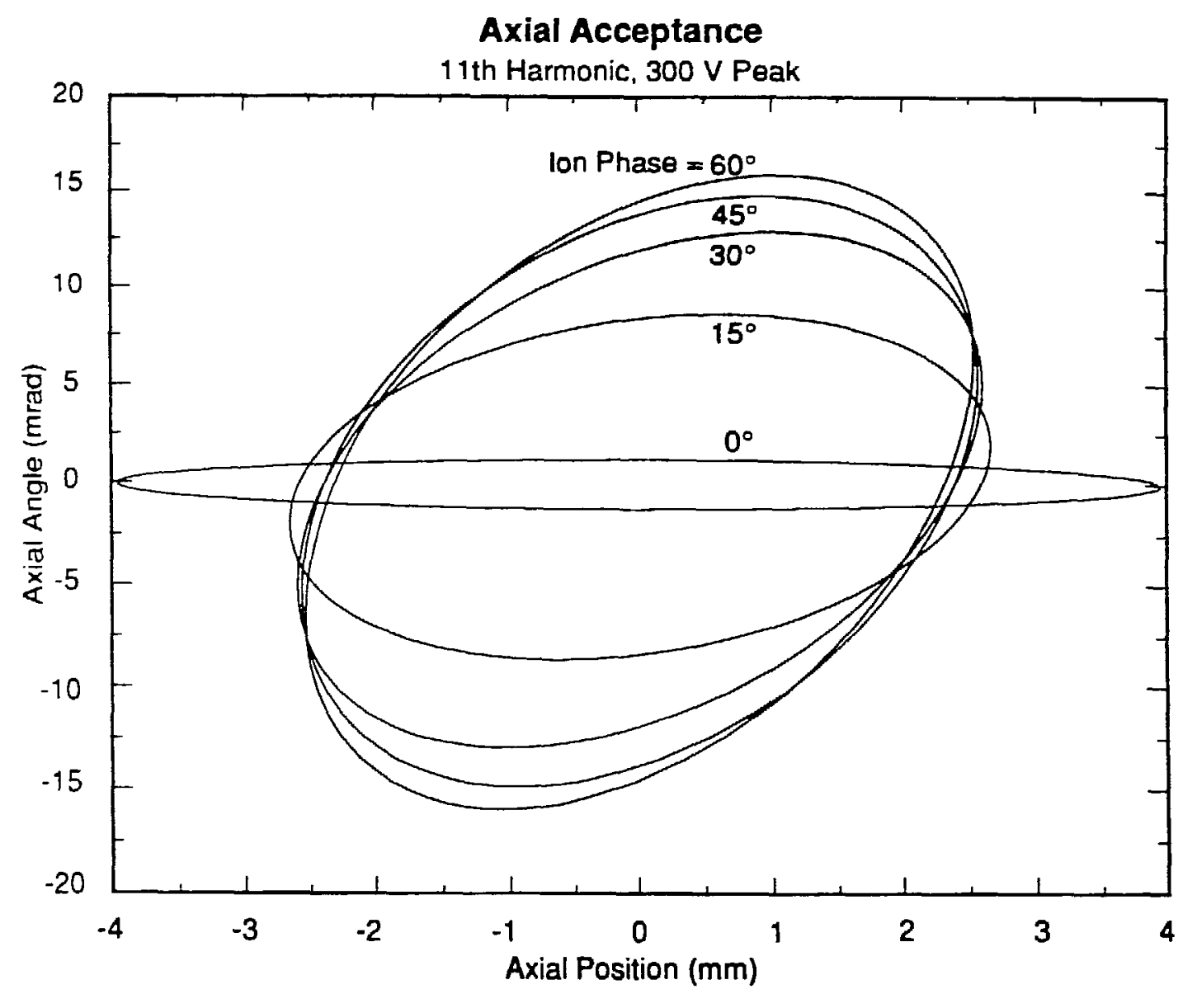

XBL $8911-4177$

Figure 2.8a Axial acceptance of cyclotron at 11 th harmonic and $300 \mathrm{~V}$ peak, parametrized by ion phase. (XBL 8911-4177) 
Axial Acceptance

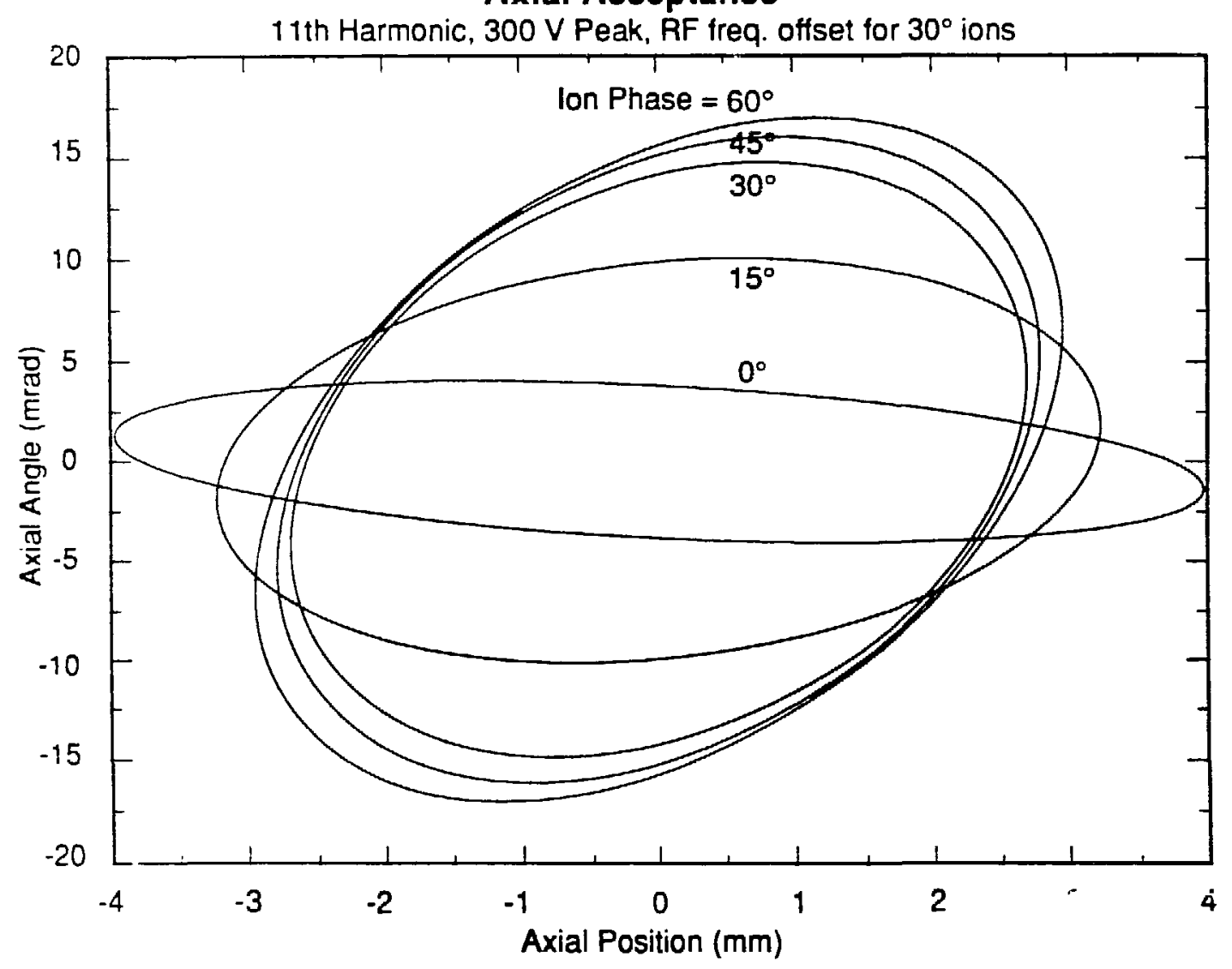

XBL 8911-4178

Figure $2.8 \mathrm{~b}$ Axial acceptance of cyclotron at 11 th harmonic and $300 \mathrm{~V}$ peak, with RF frequency offset for $30^{\circ}$ ions. (XBL $8911-4178$ ) 


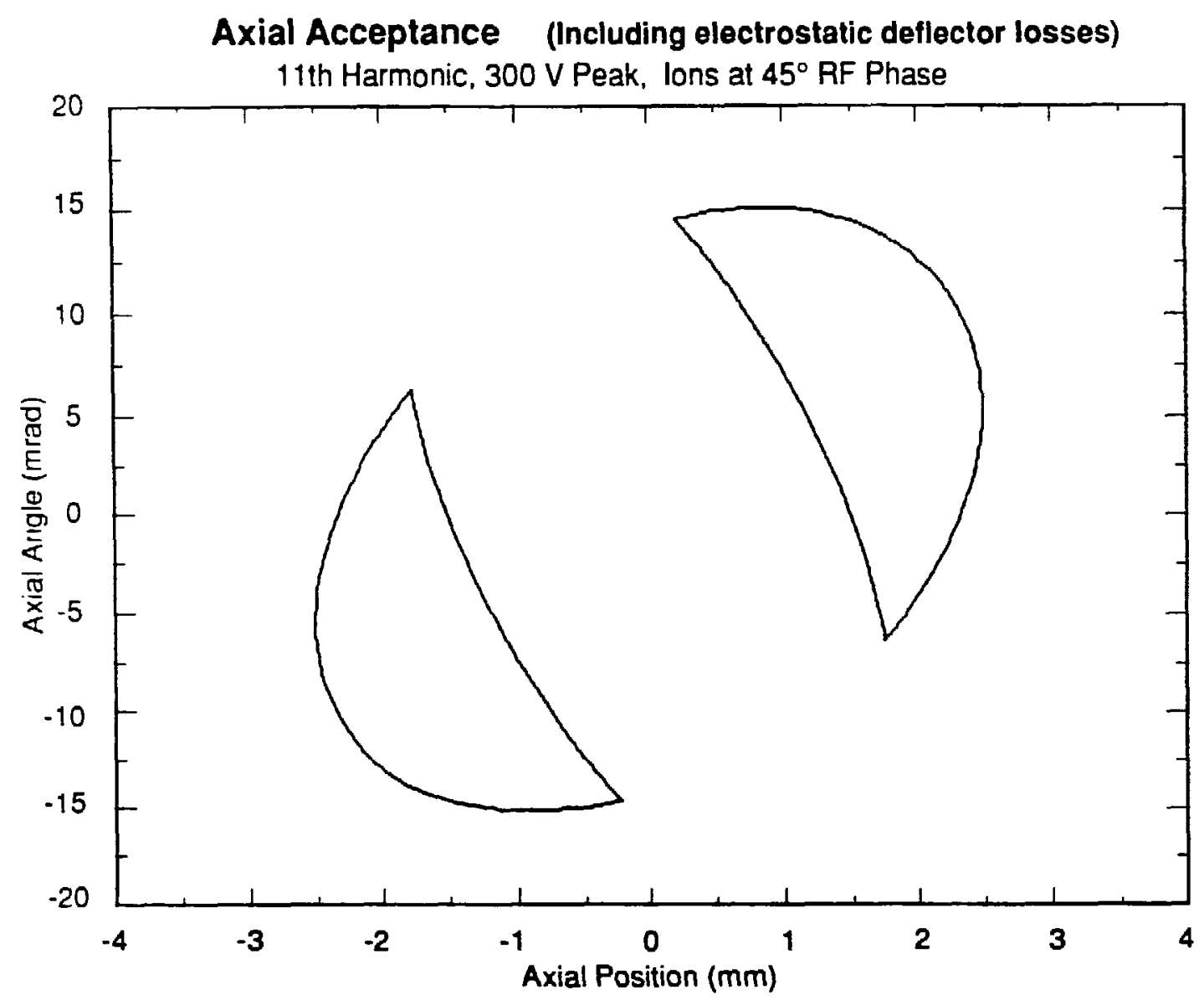

XBL $8911-4179$

Figure 2.8c Axial acceptance of cyclotron at 11 th harmonic and $300 \mathrm{~V}$ peak for $45^{\circ}$ ions, including the effect of losses due to first turn clearance. (XBL 8911-4179) 


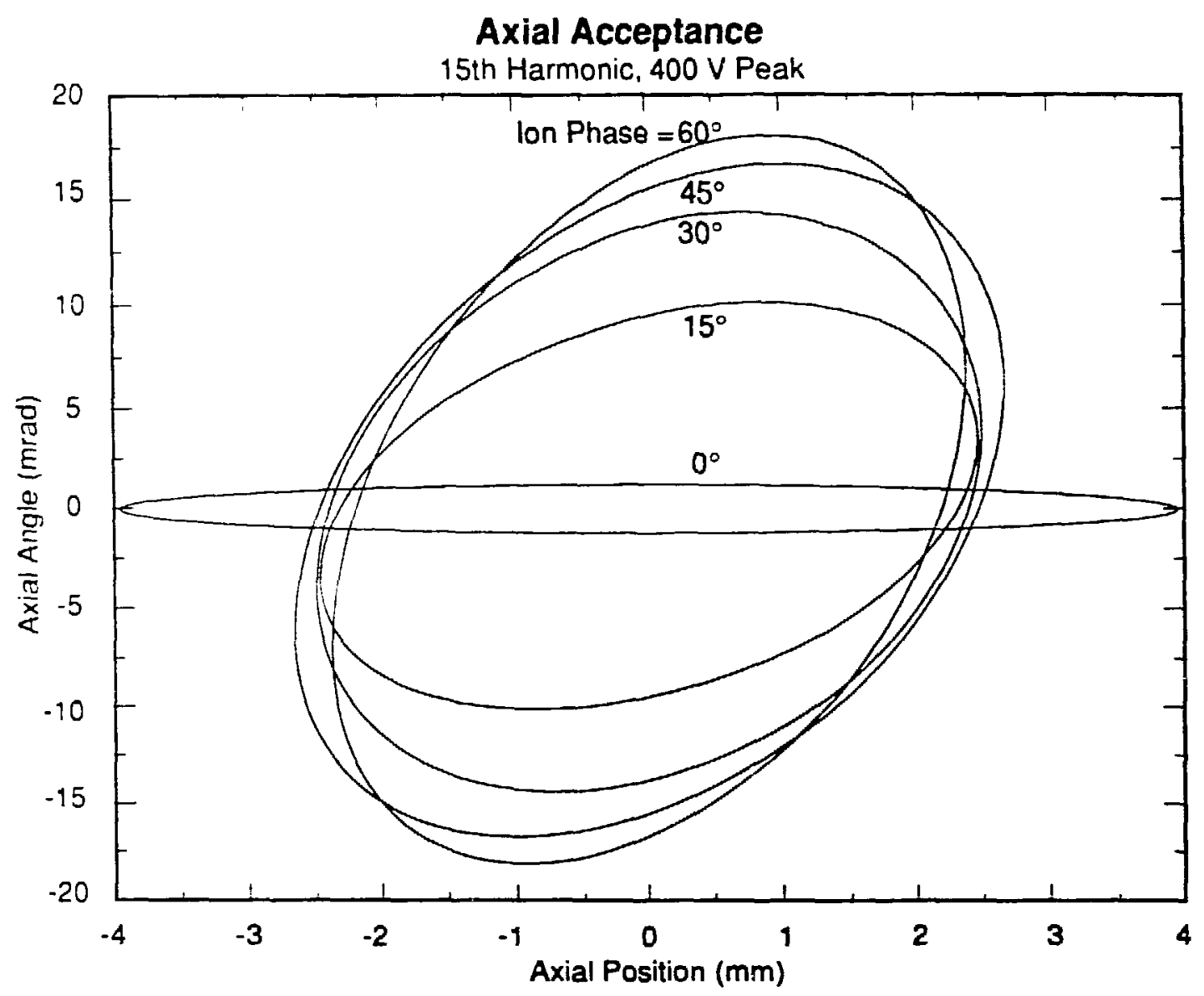

XBL 8911-4180

Figure $2.8 \mathrm{~d}$ Axial acceptance of cyclotron at 15 th harmonic and $400 \mathrm{~V}$ peak, parametrized by ion phase. (XBL 8911-4180) 


\section{ION PRODUCTION AND INJECTION}

\subsection{Overview}

A beamline transports the beam from the ion source to the cyclotron, shown schematically in Fig. 3.1 and pictorially in Fig. 3.2. An einzel lens captures and slightly focuses the highly divergent output of the ion source. An electrostatic quadrupole lens further focuses and steers the beam into the object slit of a Wien filter. The Wien filter (a velocity selector) performs the dual purpose of reducing the ion load entering the cyclotron (including elimination of the high energy tails from ${ }^{12} \mathrm{C}$ and ${ }^{13} \mathrm{C}$ ) and allowing ion source output to be monitored. It focuses the mass 14 beam from the object slit to an image slit, deflecting the mass 12 and 13 ions to a Faraday cup to provide normalization for ion source output fluctuations. The mass 14 beam (containing ${ }^{14} \mathrm{C}$ and molecular ions such as ${ }^{13} \mathrm{CH}$ ) is focused and steered into the cyclotron with a combination of four electrostatic quadrupole lenses. The beam is injected into the cyclotron radially using electrostatic deflection channels and an electrostatic mirror.

A computer program was written to simulate the optical performance of the beamline and cyclotron injection, based on the optical matrices given in Appendix E. This program allows easy examination of the first-order optical properties of the system. Either an individual ray or the beam envelope may be traced through the system. This allowed adjustment of beamline composition and geomerry, leading to the beamline shown in Fig. 3.1 with the beam envelope shown in Fig. 3.3.

In addition to these first-order optical analyses, aberrations caused by higher order effects must be considered. For elements which are symmetric in the off-axis dimensions (e.g. einzel and quadrupole lenses) even-order aberrations cancel; the lowest-order aberration remaining is third order. For asymmetric elements (e.g. Wien filters) there are second-order aberrations as well. 


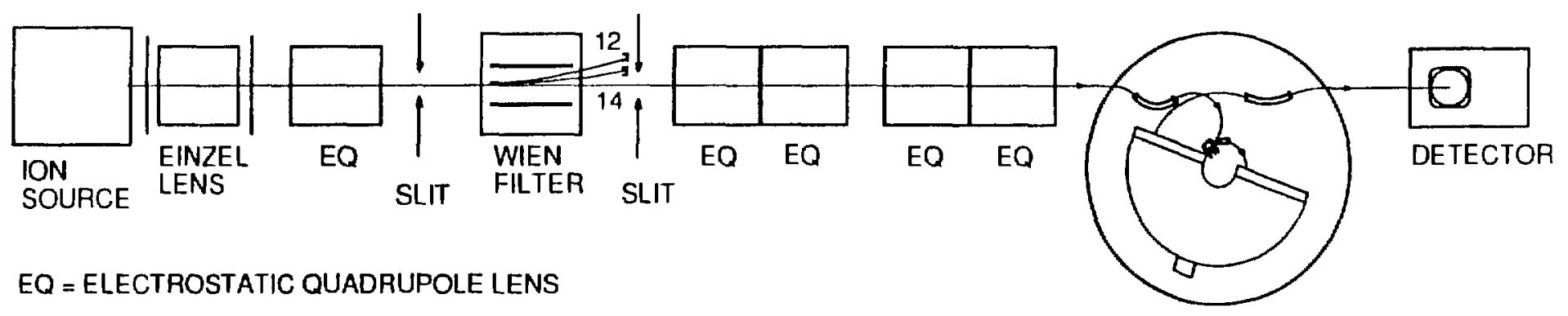

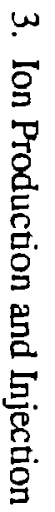

Figure 3.1 Cyclotron system schematic, showing injection beamline. (XBL 874-1819) 


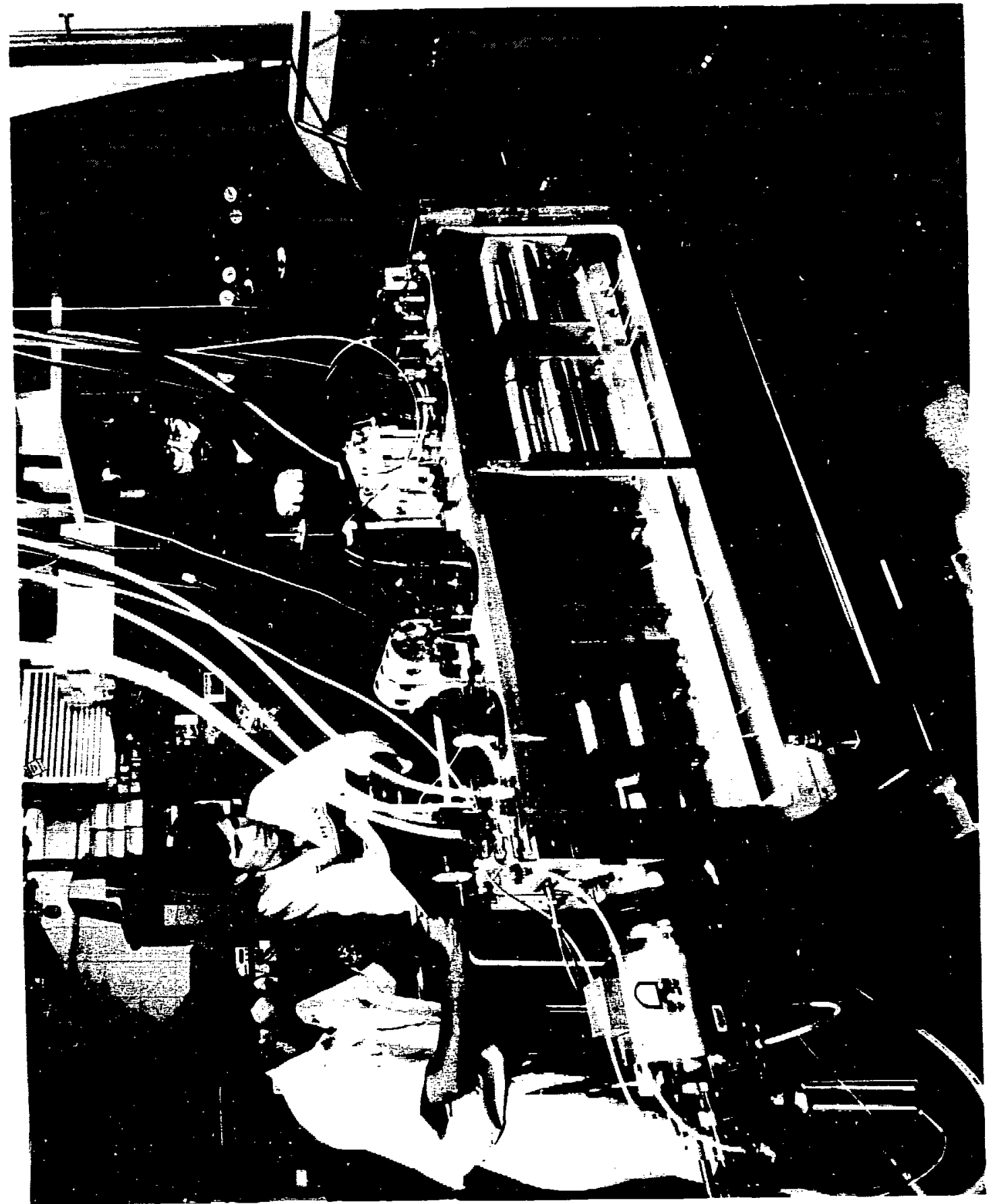

Figure 3.2 Cyclotron system, showing injection beamline. (CBB 888-8200) 


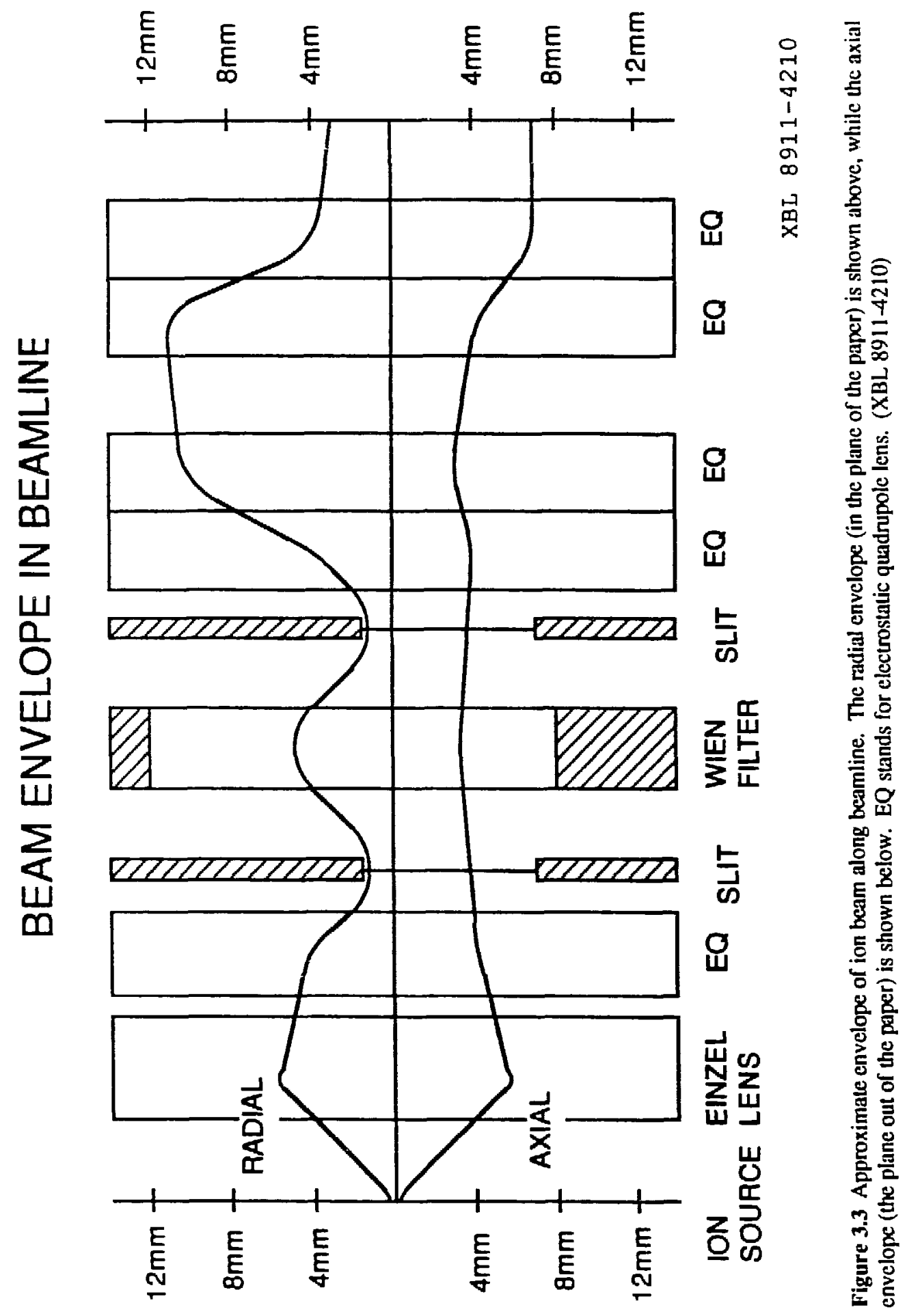


Of the third order geometrical aberrations, spherical aberration is the most important (Moore, Davis and Coplan, 1983, §5.2), although chromatic aberration is also somewhat important. The spherical aberration coefficient $\mathrm{C}_{\text {so }}$ may be defined as:

$$
\Delta r_{o}=C_{s o} \alpha_{o}^{3}
$$

where $\mathrm{C}_{\mathrm{so}}$ is the spherical aberration coefficient referred to the object, $\alpha_{0}$ is the angle of an ion which starts on axis at the object plane, and $\Delta_{0}$ is the aberration in the object plane (the apparent off-axis origin of the ion in the object plane). (El-Kareh, 1970, vol. 2, §10) This may be referred to the image plane very simply:

$$
\Delta r_{i}=M C_{s o} \alpha_{o}{ }^{3}
$$

where $\Delta r_{i}$ is the aberration in the image plane and $M$ is lens magnification.

In the beam injection system, aberrations are primarily due to the einzel lens, with the Wien filter also contributing somewhat. The quadrupole lenses contribute negligibly to the overall aberration.

\subsection{Ion Source}

The earlier version of the small cyclotron did not have enough current to enable ${ }^{14} \mathrm{C}$ detection at modern abundance levels (Welch, 1984a; Welch et al., 1984b). For this reason the current machine was built with an external ion source.

A Cs sputter negative ion source was purchased from General Ionex, capable of more than $20 \mu \mathrm{A}$ of $\mathrm{C}^{-}$output using graphite or $\mathrm{CO}_{2}$. The source is designed to operate with about $20 \mathrm{keV}$ extraction energy. However, this machine is designed to have only a 5 $\mathrm{keV}$ input beam energy; thus, beam properties were not well known. Extrapolating from performance at $20 \mathrm{kV}$ (Middleton, Purser, private communication), estimates were that at 5 $\mathrm{keV}$ the beam should have an emittance of about $40 \pi \mathrm{mm}$-mrad with an apparent origin over a region of about $1 \mathrm{~mm}$ diameter at about the actual sample location. These estimates are probably not extremely accurate, since there is significant loss of beam before the exit of the Wien filter.

An RF plasma source was also investigated (Walther, Leung and Kunkel, 1986a). This has the advantages of a very simple design and a low beam emittance and seems like a 
The cyclotron beamline had space for about a 6" diameter lens. After comparison of various designs an axially asymmetric lens similar to that of Riddle (1978) and Drummond (1984) was built (Fig. 3.4). This seems to work fairly well but does seem to introduce a significant amount of aberration due to the high divergence of the impinging ion beam.

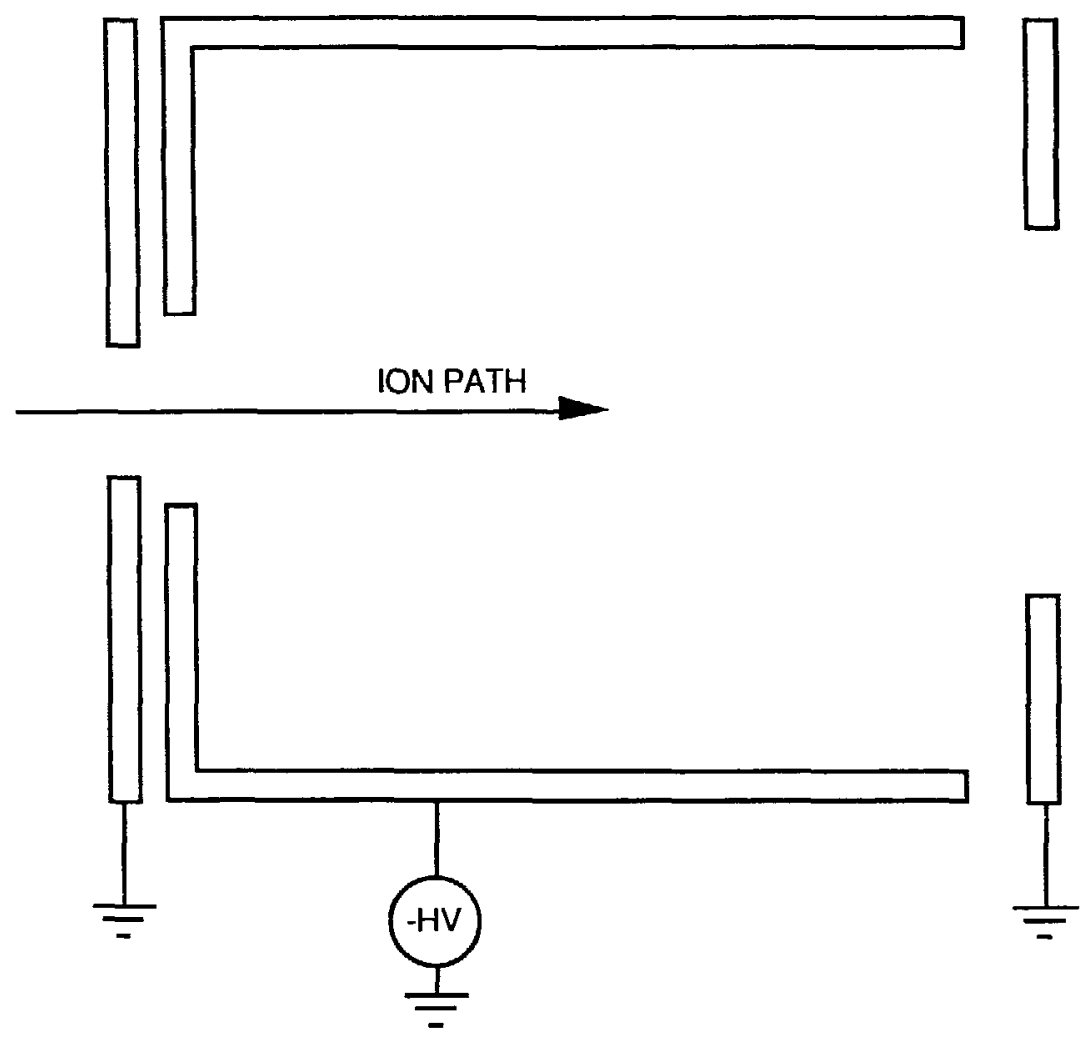

Figure 3.4 Schematic diagram of the axially asymmetric einzel lens used in the beamline.

\subsection{Quadrupole Lenses}

The cyclotron uses a number of electrostatic quadrupole lenses. Quadrupole lenses can generally be built with lower aberrations than einzel lenses and are thus preferred where it is possible to use them. They behave optically as would a saddle-surface glass lens, focusing along one axis and defocusing along the other. Since one axis is defocusing, it is generally necessary to use at least two quadrupole lenses in an optical system to avoid spreading the beam. 
Good control is needed of beam characteristics (beam position, beam width, and focal plane locations) on injection into the cyclotron. A combination of four electrostatic quadrupole lenses provides the needed flexibility. By independent adjustment of the powers of the four lenses, control is gained over the four degrees of freedom associated with beam focal properties. (These four degrees of freedom may be described as the position and size of the beam focus for each of the two dimensions orthogonal to the beam direction.) Beam steering may be accomplished by driving the quadrupoles asymmetrically; this allows adequate control of the four additional degrees of freedom associated with beam position. Custom-built power supplies allow independent adjustment of the focal power and vertical and horizontal deflections of the quadrupole lenses (Appendix C).

Quadrupole lenses, if properly designed, have relatively small aberrations. An ideal quadrupole lens has four hyperbolic poles. In practice, circular cross sections are generally used because of the simplification in manufacture. The substitution of circular poles introduces spurious multipole components, the lowest of which is a 12-pole, corresponding to a fifth-order aberration. This can be cancelled on axis by adjusting the spacing of the poles, leaving a 20-pole component (ninth-order) as the next remaining aberration. The correct pole spacing is about $r=1.15 \mathrm{a}$, where $r$ is the pole radius of curvature and $a$ is the radius of the aperture across its smallest dimension (Banford §6.4; Septier, Strong-focusing; Dawson, 1976).

These remaining aberrations are such high-order (ninth and above) that they are relatively unimportant for ions near the axis. However, lower order aberrations are introduced through other mechanisms. The fields at the ends of a quadrupole are distorted due to their fall off, introducing a third-order aberration (Septier, Strong-focusing). More importantly, the effective length of the lens varies with off-axis distance of an ion, because ions travel in different fringe fields. This also gives rise to a third order aberration (Banford $\S \S 6.5,6.8$ ). It is possible to design quadrupole electrodes with an axiallyvarying diameter to reduce these aberrations (Tiefenback, 1986); however, for this system simple cylindrical electrodes could be used without excessive aberration.

The effective length of a quadrupole lens is approximately $L+a$, where $L$ is the physical length of the poles and a is the entrance radius (Banford, 1966, §6.4). The difference in effective length on axis and at the edge of the aperture is approximately $0.15 a$ (Banford, 1966, §6.8). The effective length must vary quadratically with off-axis distance 
in order to produce a third-order aberration. Thus, realizing that lens power is proportional to effective lens length for a weak lens, one finds:

$$
P \propto \frac{1}{f} \propto L_{\text {eff }} \propto\left(1+\frac{0.15(r / a)^{2}}{L / a+1}\right)
$$

where $P$ is lens power, $f$ is focal length, $r$ is off-axis distance, $a$ is radius of lens aperture, and $L$ is physical lens length. This effect is the dominant source of aberration for a quadrupole lens.

An expression for spherical aberration may be derived from this variation in effective length. Based on the definition of spherical aberration (3.1), the effective length (3.3) and simple formulas of Newtonian optics, one finds:

$$
C_{s o}=\frac{-0.15 f^{3}}{(L / a+1) a^{2}}\left(\frac{M+1}{M}\right)^{4}
$$

where $M$ is the lens magnification.

The lenses which were built for the small cyclotron have a length of 4.0 inches, a pole diameter of 2.0 inches, and an aperture of 1.74 inches. The poles are constructed from thick-walled aluminum tubing and are sandwiched between two aluminum end plates with ceramic tubes acting as insulators and threaded rods holding the assembly together (Fig. 3.5). For these lenses, with $L \approx 4 a$ and $M=1$, we find $C_{S} \approx 0.48 \mathrm{f}^{3} / \mathrm{a}^{2}$ from (3.4). The aberration of the quadrupole lenses was found to be relatively unimportant; although the beam becomes quite large in the quadrupoles (Fig. 3.3), there is virtually no loss of beam in traveling from the Wien image slit to the cyclotron's injection channel. 


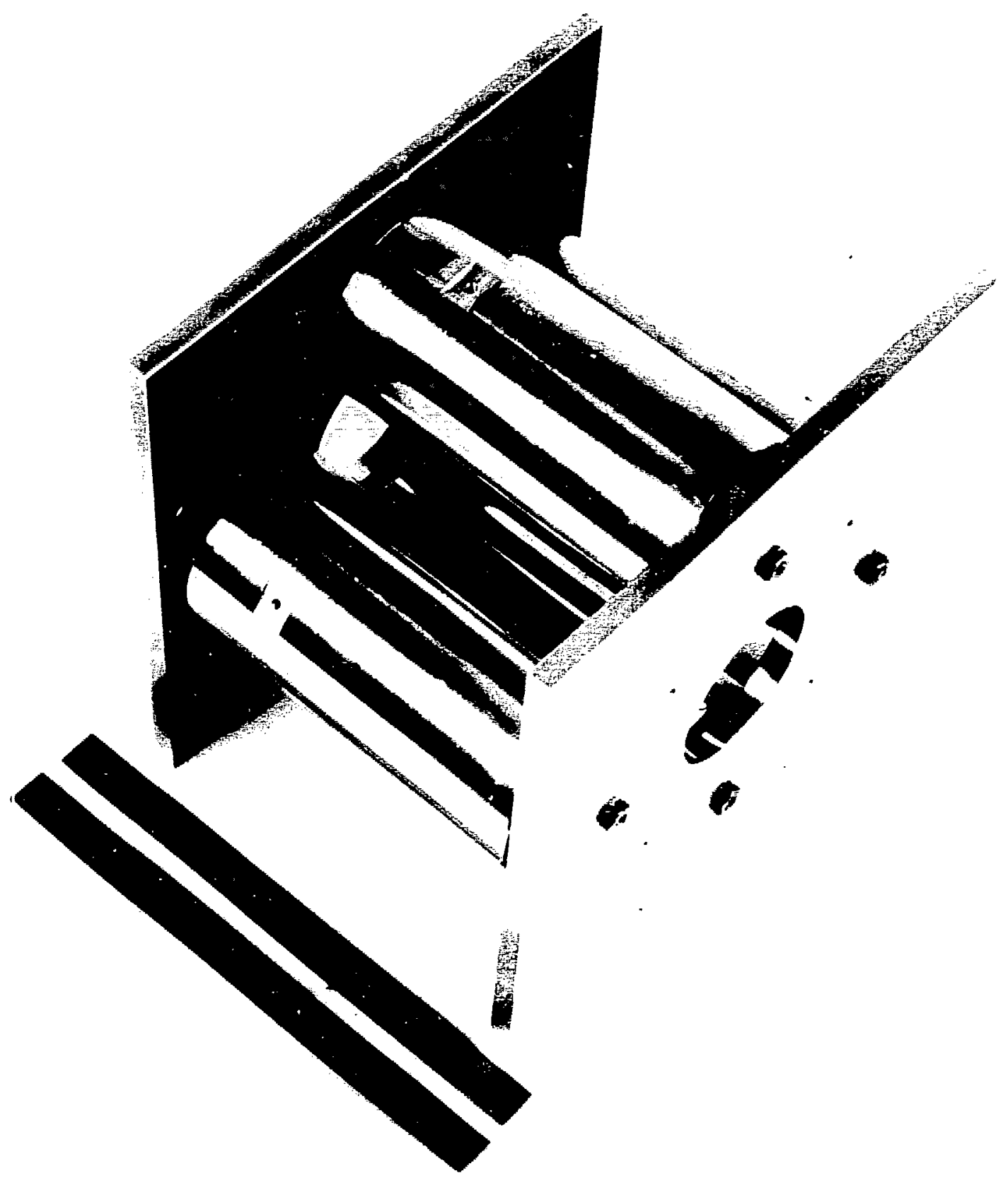

Figure 3.5 Quadrupole lens as used in the injection beamline. (CBB 874-3103) 


\subsection{Wien Filter}

A Wien filter is composed of an electric and a magnetic field which are orthogonal to one another. The ion beam travels along an axis orthogonal to both. The electric and magnetic fields are adjusted to pass the desired species undeflected; any species with incorrect velocities are deflected away from the main path. This is also known as a crossed-field separator or a velocity selector. It can be shown that such a device is truly velocity selective, independent of ion energy (Appendix D).

Wien filters can be designed in a number of ways. A Wien filter will have a focusing effect in the plane normal to the magnetic field direction. If it has a uniform magnetic field and flat magnet ends it will exert no focusing effect in the direction of the magnetic field. If focusing is needed in both dimensions, the magnet ends may be slanted or the magnetic field may be given a linear gradient (Seliger, 1972; Galejs and Kuyatt, 1978).

In the small cyclotron these complications were unnecessary. The Wien filter is composed of a slit, a drift length, the crossed field region, a second drift length and a second slit. The length of the crossed field region is such that:

$$
L \cong \frac{\pi}{2} r_{c}
$$

where $r_{c}$ is the nominal cyclotron radius for particles in the crossed field region. The two drift lengths are each about $r_{C}$ in length. This establishes a focus at the second slit (Appendix D).

The separation of off-mass ions at the second slit is given by:

$$
x_{\text {tot }} \cong r_{c} \frac{d m}{m}
$$

Once such a filter is installed, the desired beam must be imaged at the output slit. In general, it will not be imaged exactly at the slit due to measurement and assembly errors. It is of course possible to remove the filter and make mechanical adjustments. But it is also possible to adjust the focus by changing the beam energy (velocity). If the beam energy is changed a small amount at the ion source, the focal position moves according to:

$$
d z=\left(\frac{\pi+2}{2}\right) \frac{d T}{T} r_{c}
$$


where $d z$ is the change in focal position, $r_{C}$ is the nominal cyclotron radius $(=m v / q B)$ inside the Wien filter, $T$ is nominal beam energy, and $d T$ is the change in beam energy.

If the beam energy is changed a small amount only in the Wien filter (by imposing asymmetric voltages on the electric field plates), the focal position moves according to:

$$
d z=\left(\frac{\pi n}{2}\right) \frac{d T_{\text {in }}}{T_{\text {in }}} r_{c}
$$

where $n$ is the "refractive index", equal to $V_{\text {outside }} / V_{\text {inside, }}$ and $T_{\text {in }}$ is the beam energy inside the Wien filter.

The above approximations are useful for small fractional changes in energy. For larger changes in energy, as is used in this system, the more fundamental equations must be used (Appendix D). It should also be noted that adjusting the beam energy will affect the separation of off-velocity ions.

A symmetric optical element such as a quadrupole lens or einzel lens will have no second-order aberrations due to symmetry about the beam axis. However, a Wien filter is not symmetric and has second-order aberrations which must be considered. These aberrations seem to arise as a result of the fact that the ion trajectories are cycloids rather than parabolas and may be calculated by integrating the equations of motion for an ion traversing the filter.

This origin of this second order aberration may be seen qualitatively by looking at the situation in the following way. An ion whose angle carries it to a region of higher potential energy in the electric field of the Wien filter travels slower and spends longer in the filter than does an ion traveling along the optic axis, while an ion with the opposite angle goes to a lower potential and traverses the filter more quickly. This tends to increase the effect of the filter for the first ion and to reduce it for the second, causing hoth to deflect away from the image point in the direction of the electric force of the Wien filter.

A computer program was written to integrate the motion of an ion through a Wien filter to numerically calculate the magnitude of these aberrations. For our design, where the drift length $d=r_{C}$ and the field length $L=\frac{\pi}{2} r_{c}$, the aberration is $\Delta x=6 \alpha^{2} r_{c}$, while for a design where $d=0$ and $L=\pi r_{c}$, the aberration at the focal plane is $\Delta x=3 \alpha^{2} r_{c}$, where $\Delta x$ is the separation of the ion from the focal point measured in the focal plane and $\alpha$ is the ion's incident angle through the Wien filter object slit, in radians. 
The effects of this aberration may be controlled by limiting the angles of incident ions. The beam should not be focused to an extremely narrow ribbon at the slits, because the beam's angular spread will grow as a consequence of conservation of beam emittance. It is also advisable to physically limit the maximum angles which can traverse the filter; this may be done by inserting a field aperture at a point optically conjugate to the entrance slit. For our design, where $d=r_{c}$ and $L=\frac{\pi}{2} r_{c}$, this consists of merely inserting an aperture at i.he entrance to the magnetic field region.

The Wien filter in this system has been built with samarium cobalt permanent magnet material and a soft steel yoke, placed entirely in vacuum (Fig. 3.6). The magnetic field is $4.2 \mathrm{kG}$ over an effective length of $12.0 \mathrm{~cm}$, with a width of about $4.7 \mathrm{~cm}$ and a gap of about $2 \mathrm{~cm}$. According to calculations by Dr. Klaus Halbach (private communication), this should produce a field uniform to better than $0.2 \%$ over a $1 \mathrm{~cm}$ by $1 \mathrm{~cm}$ central region. Magnetic field shunts cause the field to drop quite rapidly at the ends, falling from $90 \%$ to $10 \%$ of its maximum value in less than $2 \mathrm{~cm}$.

The electric field is produced between tw'o parallel plates about $2.5 \mathrm{~cm}$ apart. The plates are narrow (less than $2 \mathrm{~cm}$ ) and the conductive magnet pole pieces are in very close proximity. The combination of these two factors would lead to severe electric field distortion unless something were done to deal with this.

A simple design was tried using low conductivity sheets (about $300 \mathrm{M} \Omega$ per square) to connect the two electric field plates on their edges (Bertsche et al., 1987, 1988). This should establish a linear electric field gradient at the edges of the electric field region, producing a nearly perfect parallel electric field inside. This scheme worked well at first, but the filter performance gradually degrader and began to work very poorly at high currents, probably due to charging of the sheets.

The electric field plates were rebuilt more carefully with three discrete metallic electrodes on each edge. Great care was taken to shield all insulators from the beam and to use two fringing field shunts at each end (Wollnik, 1987). Since ions spend more time near the center of the filter, electric field uniformity is more important near the center than near the edges. Hence the electrode dimensions were adjusted to provide a more uniform field near the center of the filier at the expense of field uniformity near the piates. The electric field assembly is shown in Fig. 3.7. 


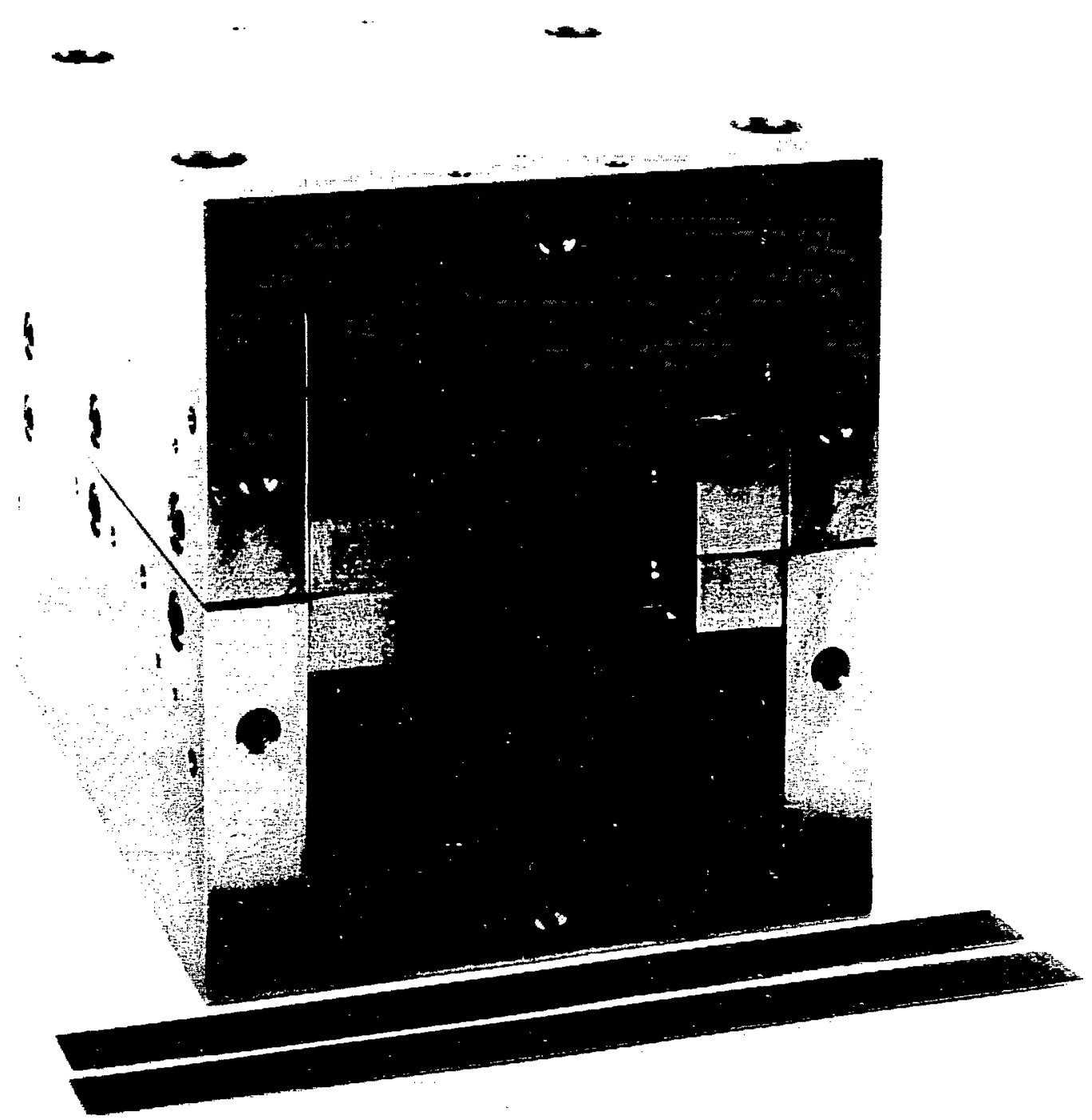

Figure 3.6 Magnet assembly used in Wien filter. The entire assembly is in vacuum. (CBB 874-3087) 


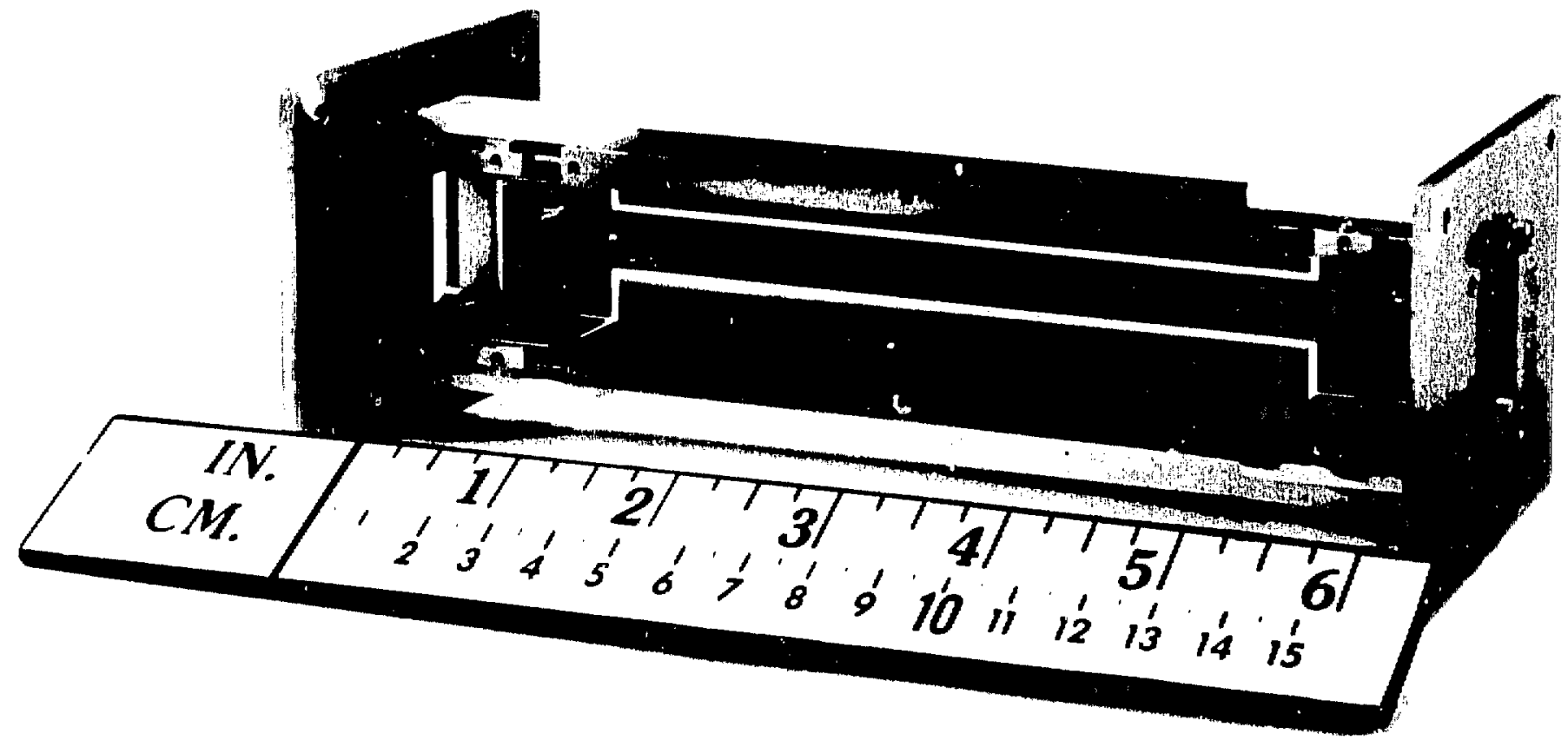


The electric potentials are applied asymmetrically in this design, with one electric field plate grounded and the other operated at a high negative voltage (about $2500 \mathrm{~V}$ ). This slows the incident $5 \mathrm{keV}$ beam significantly, shortening the necessary length of the Wien filter since ions spend more time in the crossed field region.

The separation of masses obtained with this Wien filter is shown in Fig. 3.8, measured at low currents with a Faraday cup at the output slit of the Wien filter. This plot greatly understates the off-mass rejection of the Wien filter. Many off-mass ions are able to exit the Wien filter's image slit but do not fall in the injection channel of the cyclotron because they exit the slit with a small angular deviation. Measurements of ${ }^{12} \mathrm{C}$ taken at the detector show that off-tuning the Wien filter by one mass unit (to mass 11) suppresses current by a factor of 50 , while off-tuning by two mass units (to mass 10 ) suppresses by $10^{5}$. Thus, with the Wien filter tuned to mass 14 , very little mass 12 should enter the cyclotron.

\section{Wien Filter Mass Scan}

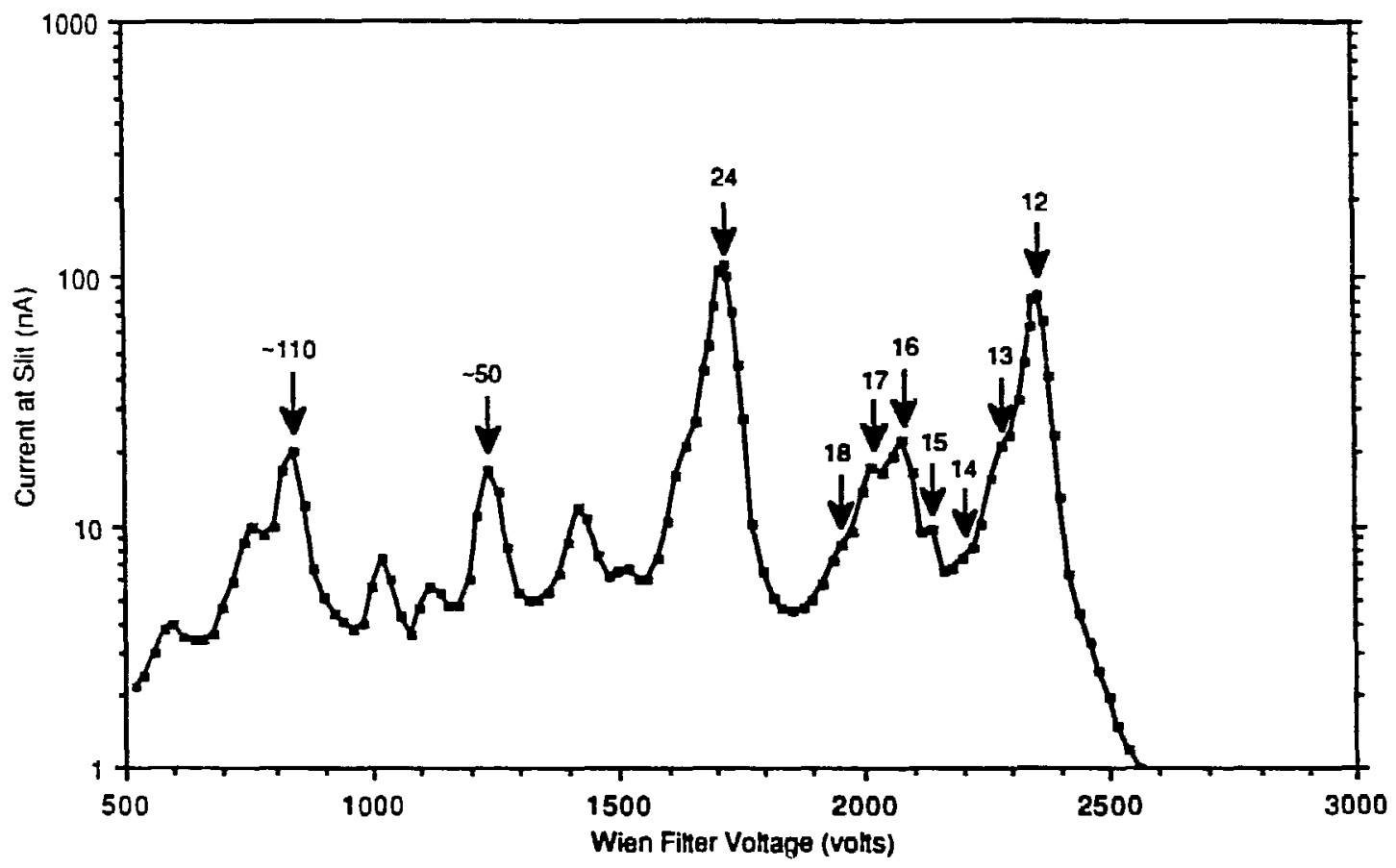

201 $0911-4214$

Figure 3.8 Mass resolution of Wien filter, measured at image slit. (XBL 8911-4214) 


\subsection{Injection}

The ion beam is injected into the cyclotron using a novel radial injection method (Morris, 1986). This was used because of concern that the more traditional axial injection would cause distortion of the magnetic field near the center and destruction of orbit isochronicity, due to the need to bore a hole through one of the magnet pole pieces. The radial injection system is shown schematically in Fig. 3.9, with a photograph in Fig. 3.10.

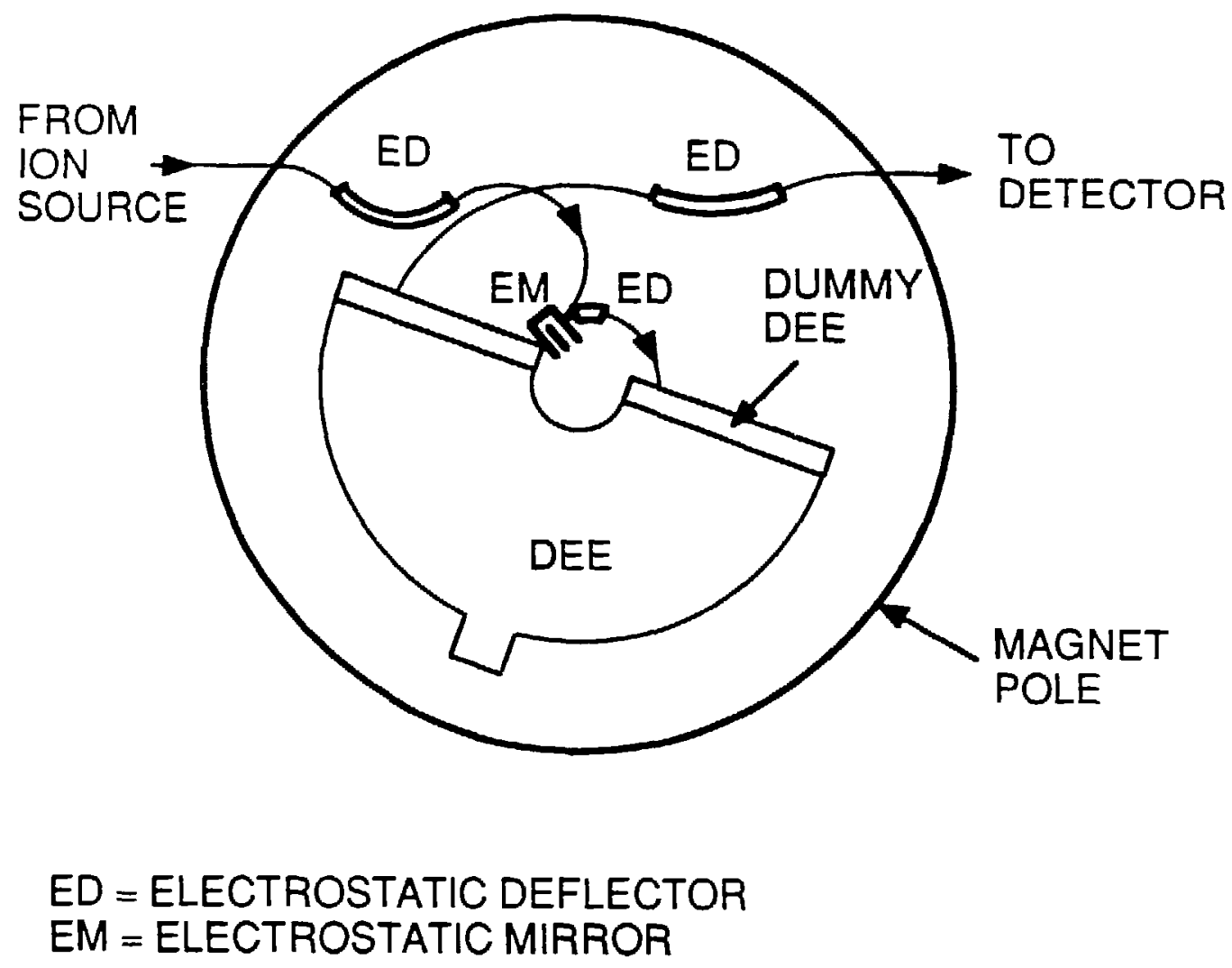

XBL 874-1818

Figure 3.9 Cyclotron schematic, showing method of beam injection. (XBL 874-1818)

Beam crosses the magnet edge at an angle, providing the proper focusing characteristics for a nearly parallel incident beam to be axially focused to a beam waist at the first dee gap crossing. Ion path and focal properties due to crossing the magnet edge must be determined by numerically integrating an ion's path through the magnet's fringing field; the standard approximations for focal properties of a magnet edge are not accurate enough (Livingood, 1969, ch. 1). 


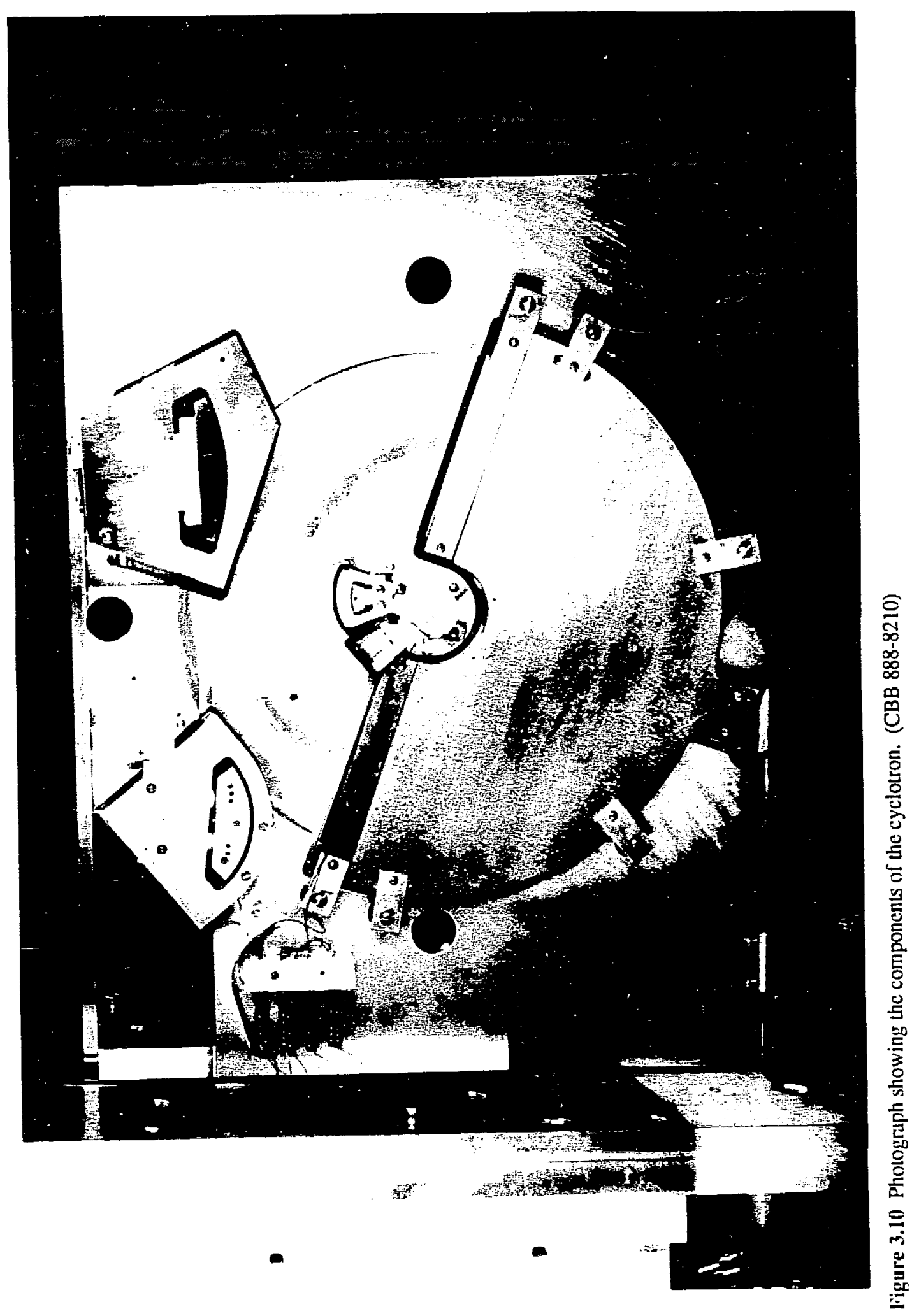


After entering the magnet region it is deflected in an electrostatic channel. This defiects the beam through about a $90^{\circ}$ angle with a $3.8 \mathrm{~cm}$ radius. The beam then curves through a $180^{\circ}$ arc, whereupon it strikes an electrostatic mirror at normal incidence. After reflection the beam travels along a circle which is nearly, but not exactly, centered on the cyclotron axis. A final electrostatic deflection channel (a $1.9 \mathrm{~cm}$ radius over about $30^{\circ}$, with a $2 \mathrm{~mm}$ gap) near the center of the cyclotron shifts the beam orbit slightly to center it and to provide clearance between the electrostatic mirror and the beam's first orbit.

Analysis of the optical properties of an electrostatic mirror in a magnetic field was done by computer simulation of ion trajectories in the mirror. The electrostatic mirror is composed of a grounded metal mesh with a flat, high voltage electrode behind it. A $5 \mathrm{keV}$ beam passing through the mesh drifts in the electric field until it reaches a $5 \mathrm{kV}$ equipotential surface, turns around, and re-exits the mesh. Due to the cycloidal paths of ions in the mirror, the apparent reflection plane is not the $5 \mathrm{keV}$ equipotential plane, but is short of this by a factor of 1.39. The focal properties of the mirror were also calculated (Appendix E). The mirror electrode was operated at a potential about $20 \mathrm{~V}$ above the incident beam energy to keep beam from reaching the electrode and depositing on it.

The focal properties of the electrostatic deflection channels were also calculated and are given in Appendix E. When the matrices for all of these elements are inserted into the beamline program mentioned earlier, the beam envelope of Fig. 3.11 is obtained. 


\section{BEAM ENVELOPES INCYCLOTRON}

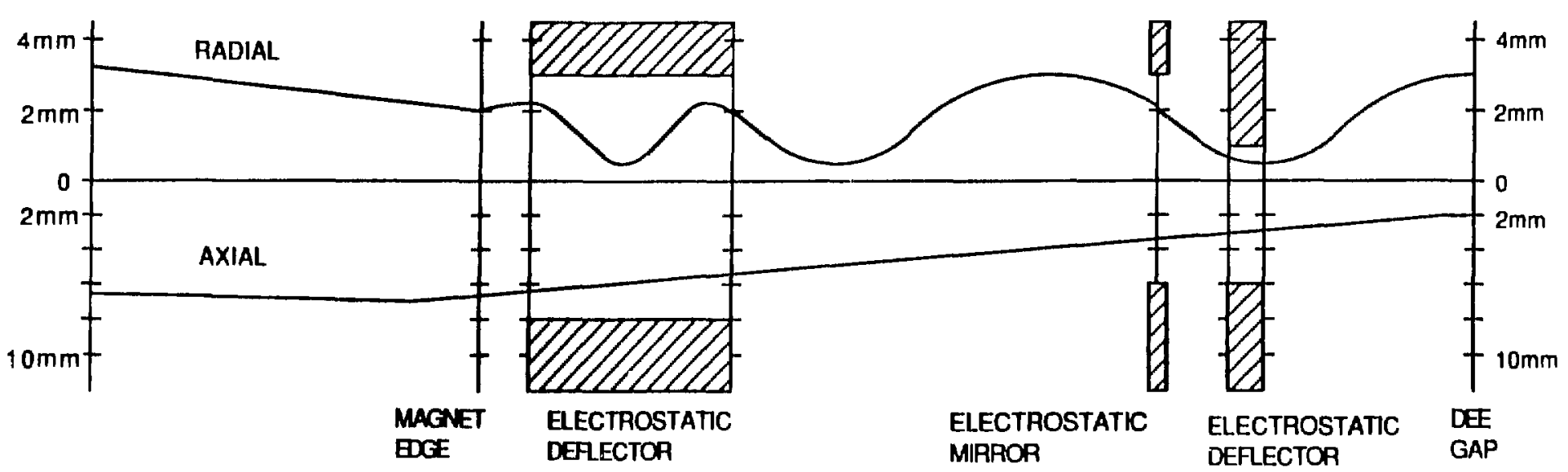




\section{ION EXTRACTION AND DETECTION}

\subsection{Extractor}

An electrostatic channel is used to deflect ions out of the cyclotron. The entrance to the channel is located $90^{\circ}$ from the dee gap (Fig. 3.9,10). This prohibits any low energy ions from entering the channel by drifting along the dee gap.

The channel has a $45^{\circ}$ arc with a $7.9 \mathrm{~cm}$ radius and a $2 \mathrm{~mm}$ gap between the two concentric electrodes. The extracted ions exit the magnetic field and strike the detector.

The 1irst order optical effects of the channel and the crossing of the B-field edge combine to produce a focus in the axial dimension with size of about $2 \mathrm{~mm}$ at a distance of about $15 \mathrm{~cm}$ from the actual edge of the magnet. At this point the radial dimension has a size of about $13 \mathrm{~mm}$.

\subsection{Detector}

A custom microchannel plate detector was designed and built to detect the output ions (Friedman, 1986; Friedman et al., 1988). This detector uses an aluminum oxide conversion dynode which is impacted by the extracted ions at glancing incidence. A number of secondary electrons are liberated from the aluminum and pulled into the microchannel plate. This allows discrimination between the $30-40 \mathrm{keV}$ output ions and any low energy ions or photons, which will release fewer secondary electrons.

The microchannel plate is operated in saturation, so that each pore which is struck will produce a saturated pulse at the anode of the microchannel plate. Thus the output charge is proportional to the number of secondary electrons collected by the microchannel plate.

The extracted ions should fall into a region about $2 \mathrm{~mm}$ by $13 \mathrm{~mm}$ at a distance of $15 \mathrm{~cm}$, where the detector is placed inside a magnetic shield made of magnet-grade sof steel. Ions strike the detector's aluminum dynode with an incident angle of about $12^{\circ}$. This angle reduces its area of $19 \mathrm{~mm}$ by $13 \mathrm{~mm}$ to a projected area of about $4 \mathrm{~mm}$ by $13 \mathrm{~mm}$ which should provide a good match to the extracted beam size. At this angle, an average of 
about 17 secondary electrons should be liberated by an incident ic $\pi$; the peak of the distribution should be at 10 to 15 electrons (Friedman, 1986).

A large number of photons, presumably UV, seems to be produced in the injection channel. This is probably due to secondary electrons which are produced in the channel by incident ions, gain energy from the electric field, then strike surfaces at high velocity. Copper plated optical shields were installed to block and absorb these photons. Unfortunately, a significant amount of UV still strikes the detector. The tail of the UVinduced single electron distribution extends well into the distribution for high energy ions. Because of this it was necessary to reduce the angle at which ions strike the dynode by both physically rotating the detector and by increasing the dynode voltage to deflect beam electrically. The angle of ion beam incidence was reduced from about $12^{\circ}$ to about $6^{\circ}$, shifting the peak of the high energy ion distribution from about 10 secondary electrons to about 17 secondary electrons. This also reduced the projected area of the dynode by a factor of two, making alignment much more critical.

\subsection{Detection Electronics}

In a further attempt to reduce the interference of UV photons, pulses from the detector were gated in time (Fig. 4.1). Pulses were only accepted if they fell into a phase window of about $90^{\circ}$ width. This reduced the number of UV photons by about a factor of four, while reducing the number of high energy ions by about $25 \%$. The width of this window can not be reduced arbitrarily; ions are spread in phase due to a (small) spread in extraction energy (which is due to the radial spread in position of orbit centers, as discussed in section 2.3.2), and due to their striking the dynode in different places, which correspond to different distances from the extractor. For our design, the time spread at the detector due to the ions' velocity spread is about $\pm 6 \mathrm{~ns}$ and the time spread due to the dynode size is about $\pm 9 \mathrm{nS}$, resulting in a $\pm 11 \mathrm{nS}$ spread (adding in quadrature). For our typical operation at the 15 th harmonic, this corresponds to about $\pm 60^{\circ}$. 


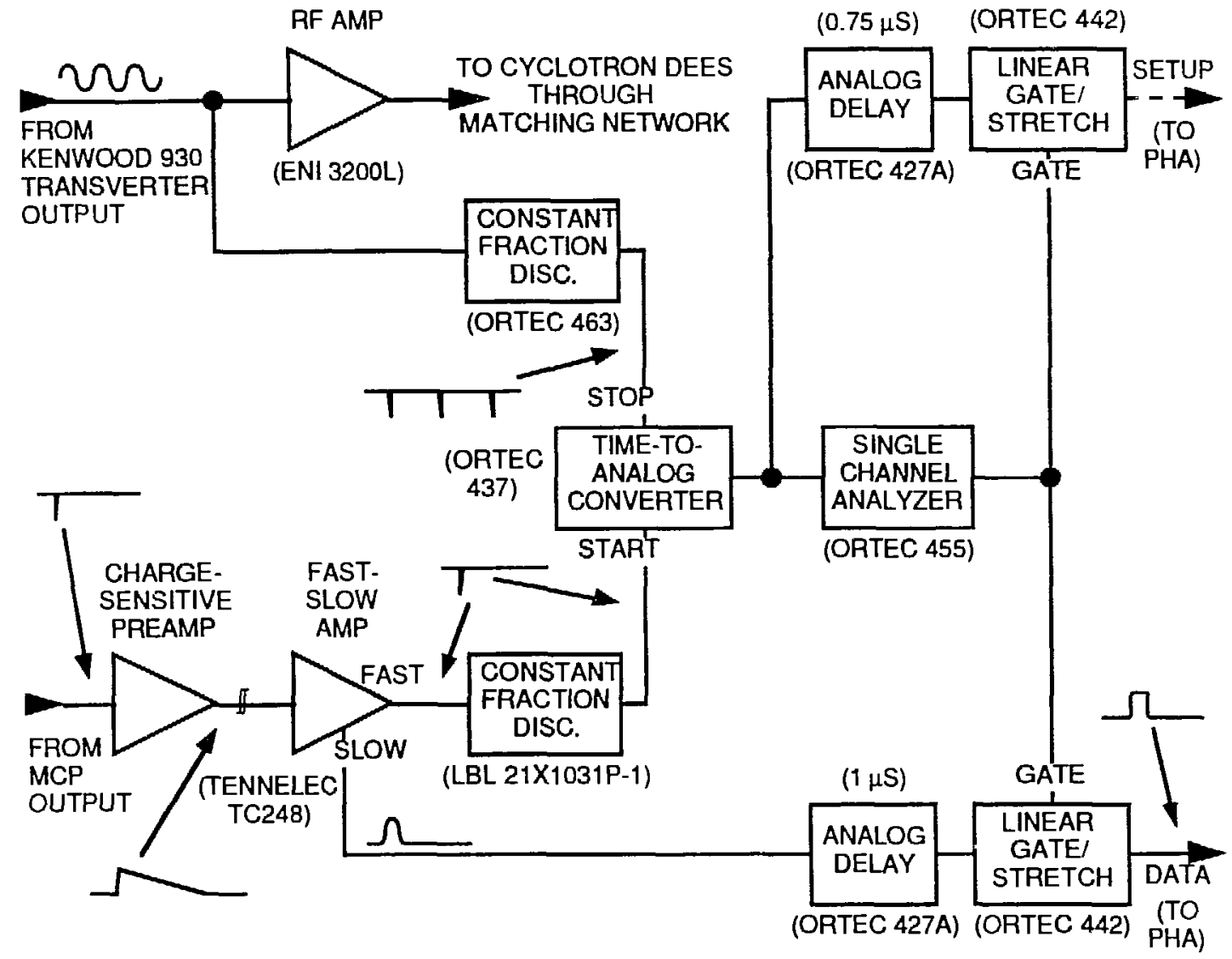

Figure 4.1 Block diagram of detection electronics. (XBL 8911-4181)

The tradeoffs involved in adjusting the width of this phase window may be seen in Fig. 4.2. Data is shown at the 15 th harmonic and $400 \mathrm{~V}$ peak for no phase windowing, a wide phase window, and a narrow phase window. Data for the "noise" curves was taken about $10 \mathrm{kHz}$ above the ${ }^{13} \mathrm{CH}$ peak, while data for the "signal" curves was taken on the ${ }^{13} \mathrm{CH}$ peak with some of the lenses in the beamline de-tuned to protect the microchannel plate detector from high count rates. 


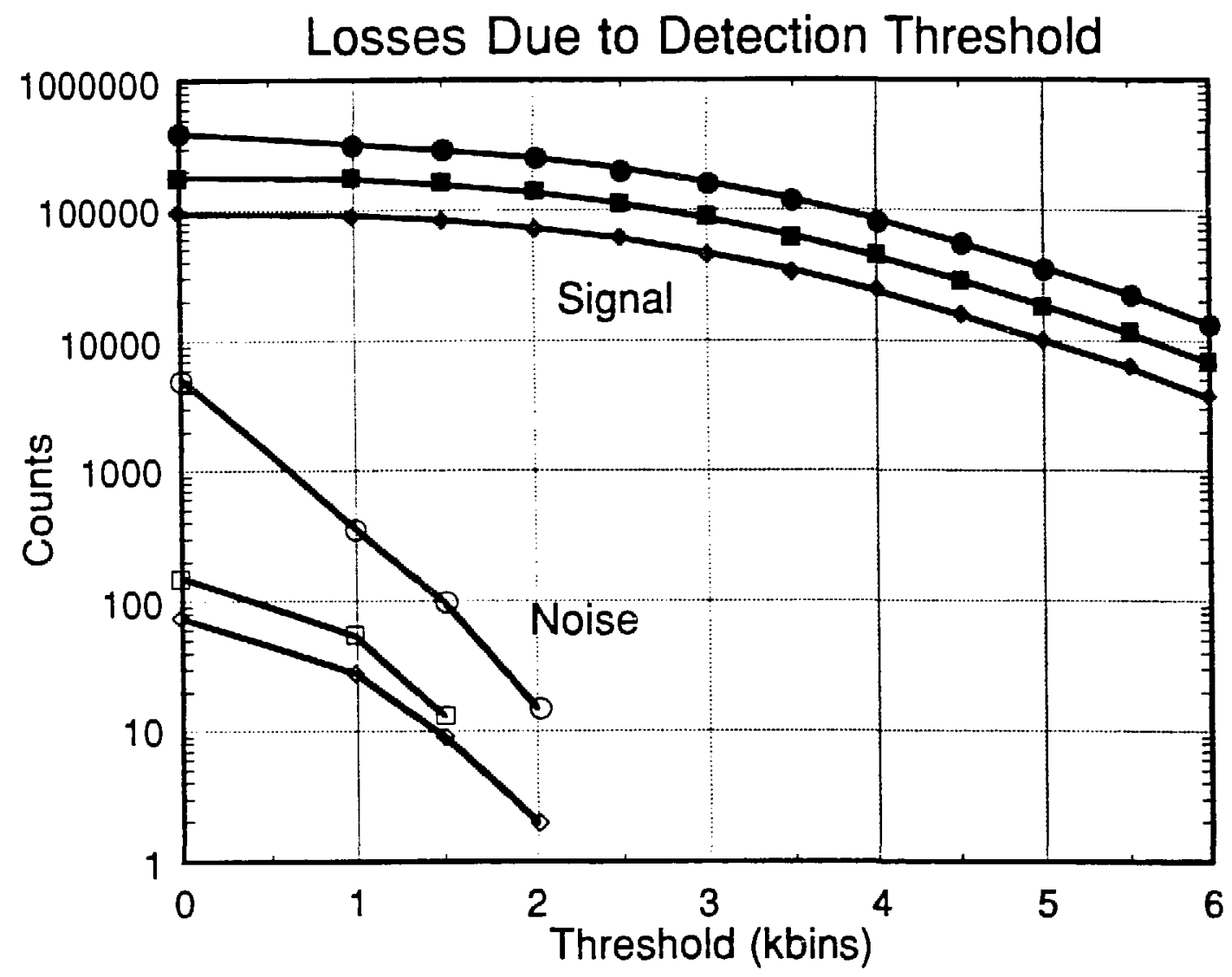

XBL $8911-4183$

Figure 4.2 Counts above threshold measured as a function of discrimination threshold. The upper curves of each set show the effects of amplitude discrimination with no phase gating, the center curves with a wide phase window (about $90^{\circ}$ ), and the lower curves with a narrow phase window (about $45^{\circ}$ ). (XBL 89114183) 


\section{SYSTEM PERFORMANCE}

\subsection{Transmission}

System losses are illustrated in Table 5.1. The transmission of the most complex part of the system (from the output of the Wien filter to the extraction channel of the cyclotron) is quite good, at about 2.5\%. A factor of 8 loss in the cyclotron would be expected due to phase acceptance alone. (Only ions with a phase between about $15^{\circ}$ and $60^{\circ}$ have enough energy gain and focusing to reach extraction radius.) Transmission through the cyclotron itself is shown in Fig. 5.1, measured with a dee probe. The abrupt drop at about $10 \mathrm{~cm}$ is due to interception of ions by the extraction channel.

Table 5.1

Transmission losses in cyclotron system.

From:

ion source

Wien furst slit

Wien second slit

injection channel

mirror

inner deflector

extraction radius

phase discrimination

amplitude discrimination

Wicn output

Wien output

ion source

* estimated
To:

Wien first slit

Wien second slit

injection channel

mirror

inner deflector

extraction radius

detector

extraction radius

detection

detection
Loss

Factor

$4 *$

5

1

2

2

10

5

1.3

4

40

$10^{3}$

$2 \times 10^{4}$

The overa!l efficiency of the system is somewhat lower than expected, with unexpected losse; seen at each end of the system. Loss at the beginning of the beamline is probably due to aberrations (and possibly some mispositioning) of the einzel lens. It should be possible to improve this performance by re-designing the einzel lens on the basis of detailed emittance measurements or computer calculations, yielding a transmission increase of a factor of 5 to 10 . 


\section{Cyclotron Losses}

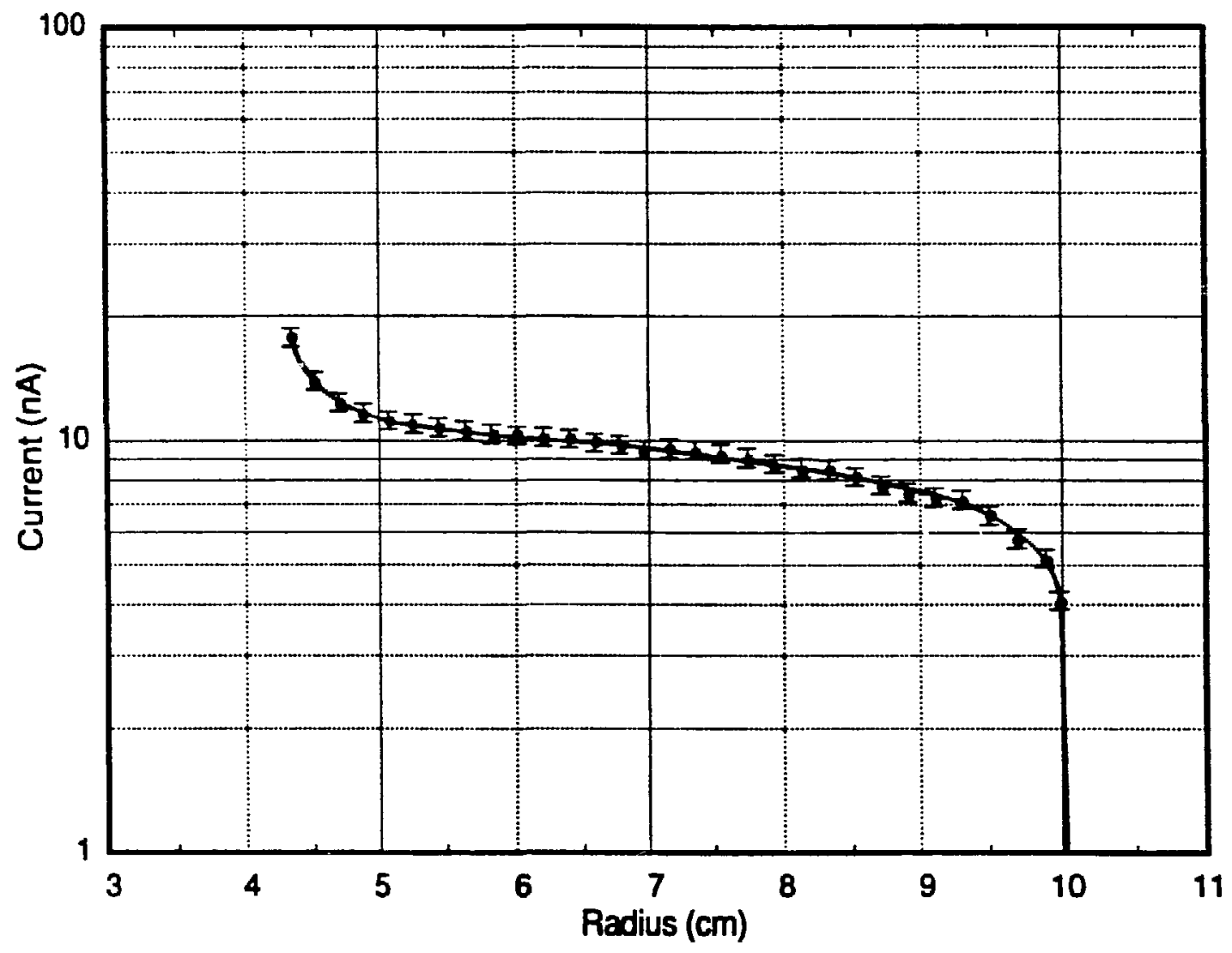

XBI $8911-4182$

Figure 5.1 Ion current as a function of radius in the cyclotron. (XBL 8911-4182)

There is also unexpected loss in ion detection due to the discrimination of ions from UV photons. The loss from extraction radius to detector is larger than expected; a factor of two would be expected (Welch, 1984a) while a factor of five is seen. This is probably due to part of the beam missing the dynode. (The dynode should be able to collect the entire beam; this loss is probably a result of minor misalignments and uncertainties in beam focus position.) A factor of four loss is due to setting a high discrimination threshold.

It may be possible to reduce these detection losses by a more thorough addition of optical absorbers, though this would probably not make a significant improvement. However, extending the size of the dynode and changing the position of the microchannel plate (to present a larger projected area to the beam) and placing the dynode at more of a 
glancing incidence (to produce more secondary electrons from each incident ion) should reduce losses both from beam missing the dynode and from the detection threshold, improving the detection efficiency by at least a factor of four.

It is also possible to add a beam buncher. This has been suggested for this system (Bertsche et al., 1987) and has been investigated in detail by Chandu Karadi (Appendix F). A beam buncher should yield another factor of four increase in efficiency.

Making all of the above improvements (einzel lens, beam buncher, and detector modifications) should boost system efficiency by two orders of magnitude. The relationship between efficiency and counting rate is shown in Appendix G.

\section{$5.2{ }^{14} \mathrm{C}$ Measurements}

The resolution of the system has proven to be adequate for ${ }^{14} \mathrm{C}$ measurements, as shown previously (Welch, 1984a; Welch et al., 1987a). The resolution peak is relatively flat over a frequency range of about \pm one part in 6000 , with very steep skirts outside this range which fall about six orders of magnitude for an offset of one part in 10,000 (Fig. $5.2)$.

At mass 14 , the dominant species are ${ }^{12} \mathrm{CH}_{2}$ and ${ }^{13} \mathrm{CH}$. The relative proportion of these two species is quite variable, depending on residual gases in the vacuum chamber and on ion source output current. (As source output is increased, the sample heats up and ${ }^{12} \mathrm{CH}_{2}$ production drops in relation to ${ }^{13} \mathrm{CH}$.) A typical scan of these peaks is shown in Fig. 5.2. 


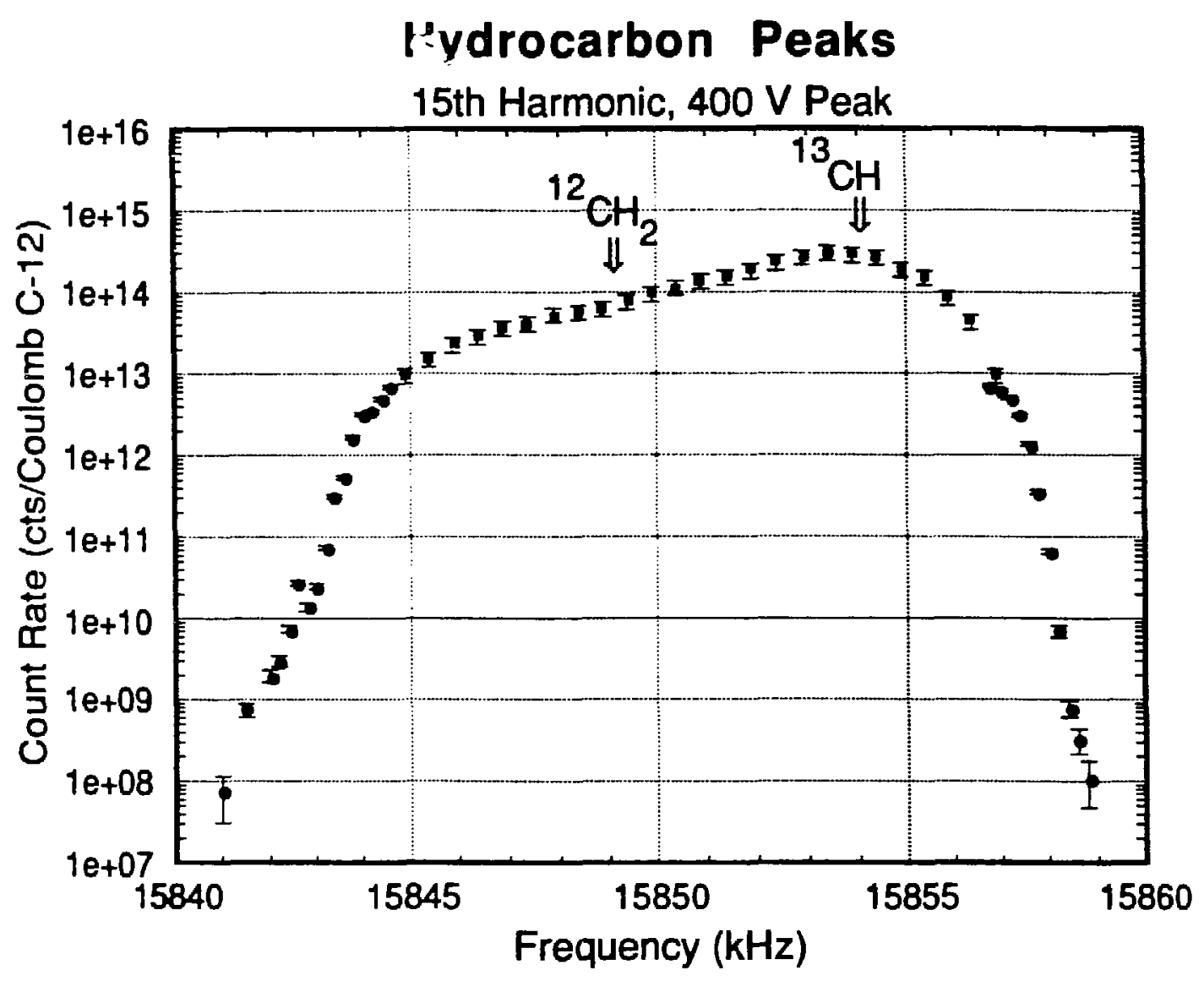

XBL $8911-4222$

Figure 5.2 Mass 14 hydrocarbon peaks. Operation is at the 15 th harmonic with $400 \mathrm{~V}$ peak RF. RF frequency is along the horizontal axis, with count rate on the vertical axis. (XBL 8911-4222)

A sample of modern carbon prepared by Prof. Erv Taylor from the OX-1 standard was measured in the system (Fig 5.3). The ${ }^{14} \mathrm{C}$ peak is easily identified. The shape of the peak was found by measuring an enriched sample of ${ }^{14} \mathrm{C}$ (Fig. 5.4). The peak should be at a frequency which is higher than the ${ }^{13} \mathrm{CH}$ peak by a part in 1800 , or about $8.5 \mathrm{kHz}$. Since the ${ }^{13} \mathrm{CH}$ peak is asymmetric (see Fig. 5.2), its location was mis-identified systematically by about $1 \mathrm{kHz}$, and the ${ }^{14} \mathrm{C}$ peak lies $9.5 \mathrm{kHz}$ above this "apparent" ${ }^{13} \mathrm{CH}$ peak location. At the ${ }^{14} \mathrm{C}$ peak, the count rate was about 0.2 counts per minute with an ion current of roughly $10 \mu \mathrm{A}$.

A sample of enriched carbon was also measured in the system (Fig. 5.4). This sample had been enriched roughly 15 times above modern levels in a reactor, courtesy of Dr. Frank Asaro and Dr. Helen Michel. The enrichment of this sample allowed a more 
efficient detection setting than that in Table 5.1; the amplitude discrimination setting was lowered, giving a loss factor of about 1.5 rather than 4 . The measured count rate for this sample was actually higher than expected by about a factor of 6 . It is not completely understood why this is so; it is possible that the enrichment was higher than expected, and it may be that other loss factors were reduced from the values in Table 5.1 due to the lower ion currents used for the measurements of the enriched sample. At the peak, the count rate was about 15 per minute with an ion source output of roughly $4 \mu \mathrm{A}$.

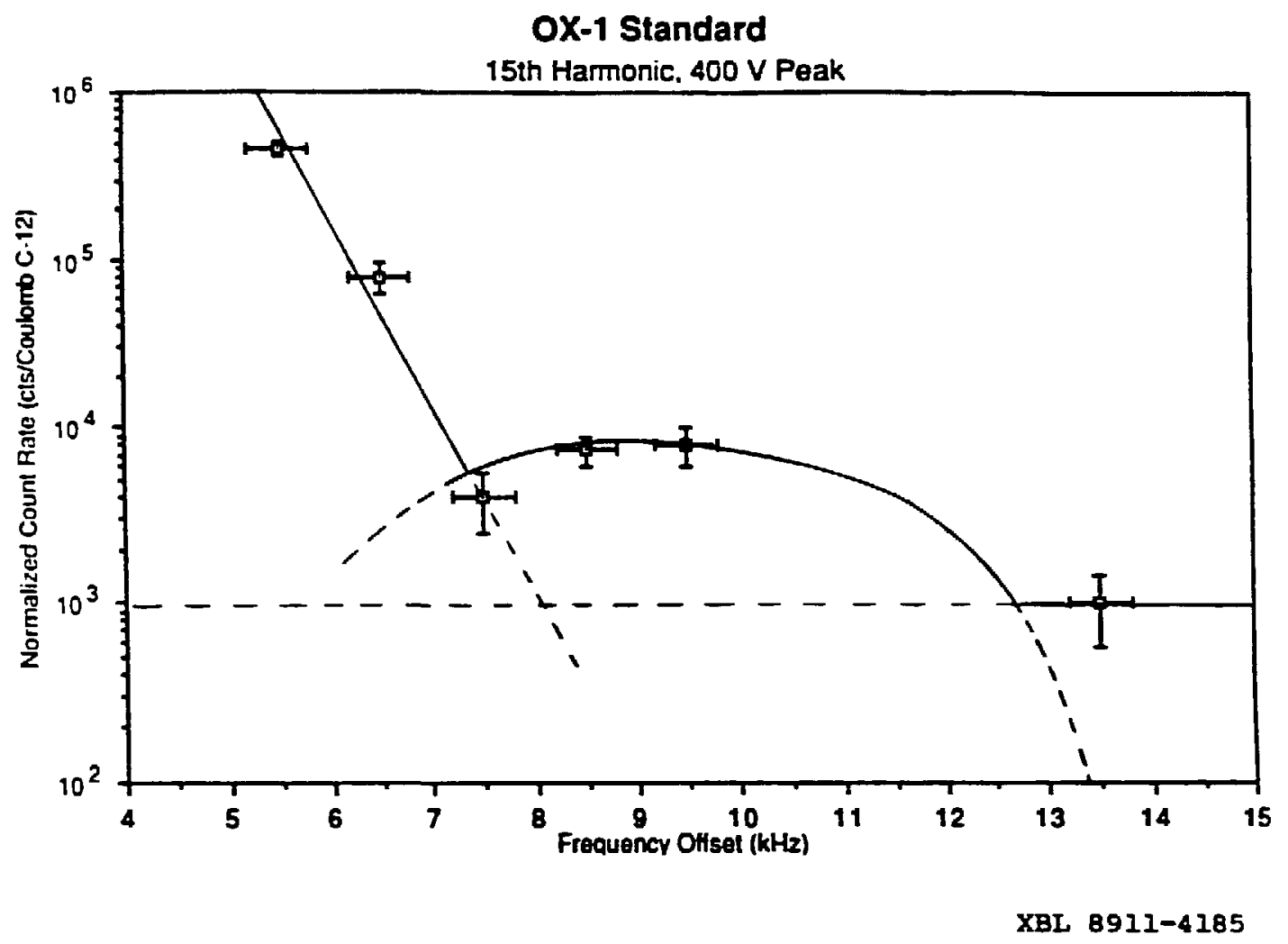

Figure 5.3 Carbon of modem age. Operation is at the 15 th harmonic with $400 \mathrm{~V}$ peak RF. RF frequency offset from the ${ }^{13} \mathrm{CH}$ peak is along the horizontal axis. The vertical axis is the normalized count rate, expressed as the counts per coulomb of ${ }^{12} \mathrm{C}$ measured at the output of the Wien filter. (XBL 89114185) 


\section{Enrlched Sample}

15th Harmonic, $400 \mathrm{~V}$ Peak

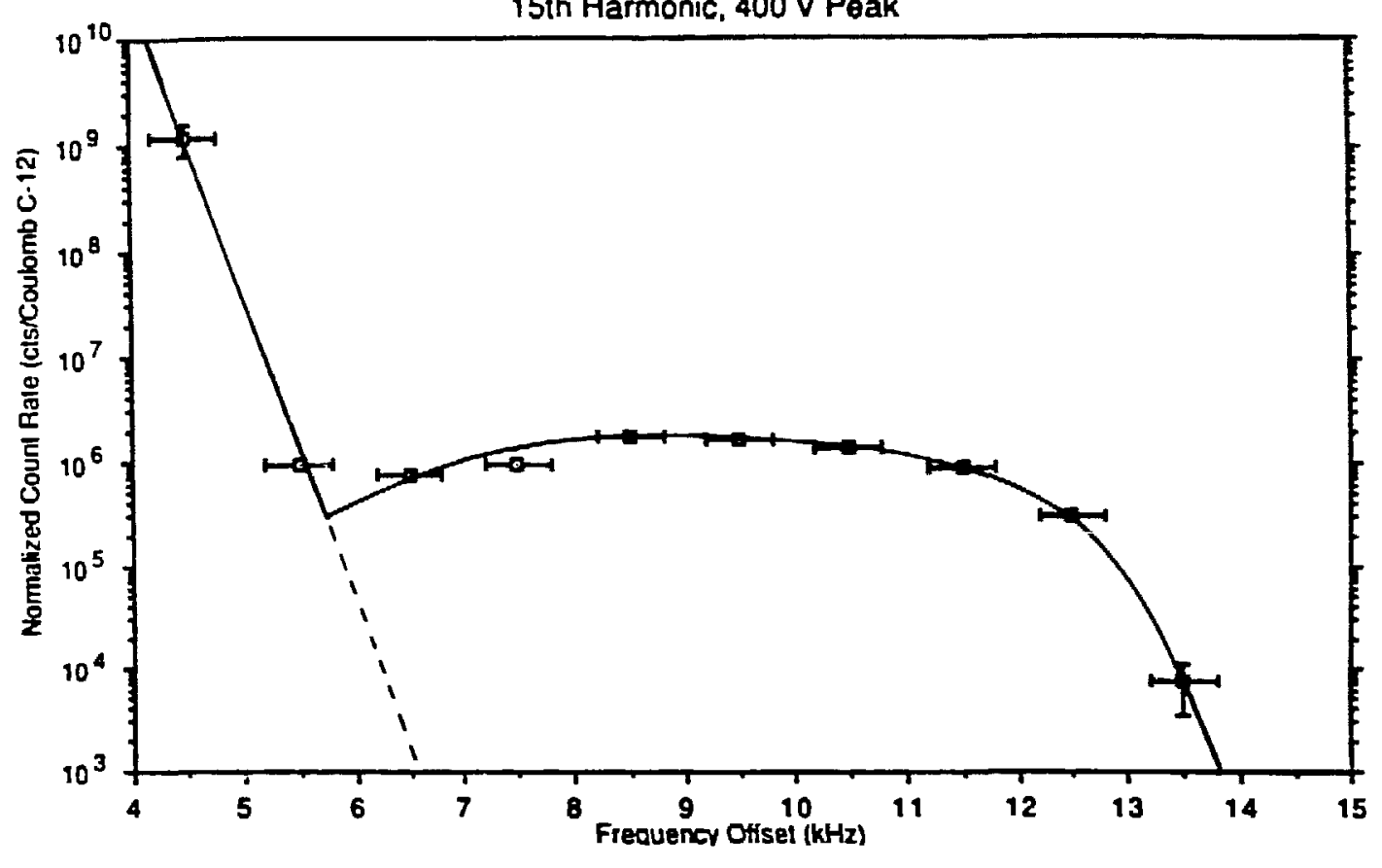

XBL 8911-4186

Figure 5.4 Enriched sample. Operation is at the 15 th harmonic with $400 \mathrm{~V}$ peak RF. RF frequency offset from the ${ }^{13} \mathrm{CH}$ peak is along the horizontal axis. The vertical axis is the nomalized count rate, expressed as the counts per coulomb of ${ }^{12} \mathrm{C}$ measured at the output of the Wien filter. (XBL 8911-4186) 


\section{APPLICATIONS AND IMPROVEMENTS}

The cyclotrino offers great potential for biomedical research. Samples with enriched levels of ${ }^{14} \mathrm{C}$ can be easily measured with a $10^{4}$ increase in sensitivity over that of conventional scintillation counting. This corslusion is based on measurements with the OX-1 standard; a factor of 5 enrichment over natural abundance would give a count rate of about one count per minute. Since a factor of $7 \times 10^{4}$ enrichment is necessary to give $1 \mathrm{dpm}$ for a $1 \mu \mathrm{g}$ carbon sample by decay counting, this represents an improvement of four orders of magnitude.

Though the device has very good sensitivity for biomedical studies, at present it is not competitive with tandem accelerators for archaeological or geological measurements. However, with improvements in the einzel lens geometry to better match beam from the ion source to the Wien filter, a factor of 5 to 10 increase in efficiency should be attainable. With improvements in the detector system a factor of about 4 should be attainable. The addition of a beam buncher should add another factor of 4 . Thus, count rates of 5 per minute withcut a buncher, or 20 per minute with a buncher should be attainable from modern carbon. This would make the system a practical tool for archaeological and geological applications.

In order to make high precision measurements, a rapid sample changing method is necessary to cancel errors due to small changes in the transmission and detection efficiency of the system. This could be provided with a rotary sample changing apparatus as is used with many tandem accelerator mass spectrometers. Rapid sample changing could also be done by making measurements with $\mathrm{CO}_{2}$ gas rather than graphite; $\mathrm{CO}_{2}$ could be injected into the ion source through a porous plug and sample switching could be done by simply switching gas sources.

It would also be possible to measure other isotopes with the cyclotrino (Appendix A). Tritium should be measurable. The nearest interfering mass is different by a part in 500 , so the resolution requirements are much more relaxed than for carbon and are easily attained. It will be much lower in abundance than is ${ }^{14} \mathrm{C}$, which may lead to some difficulties with backgrounds or count rates. Normalization of tritium to hydrogen may pose some minor difficulties since there tends to be a low level background of hydrocarbons in the vacuum. 
If the resolution of the cyclotron can be increased by a factor of about $10,{ }^{10} \mathrm{Be}$ would be measurable. This resolution increase would entail operating the system at a very high harmonic (higher than 50th), which would greatly reduce the transmission of the system. (At such high harmonics the transit time effect leads to insufficient radius increase for many ions on the first orbit.) Since the natural abundance of ${ }^{10} \mathrm{Be}$ is relatively high, this reduced transmission may well be tolerable. Be does not form negative ions, so it would be necessary to accelerate a negative molecular ion (e.g. $\mathrm{BeH}^{-}$) or to generate $\mathrm{Be}^{+}$ with a positive ion source.

A more modest increase in resolution should allow measurements of $40 \mathrm{~K}$ and ${ }^{44} \mathrm{Ti}$. This would require about a factor of 3 increase in resolution, which can be achieved by doubling the harmonic to 31 st (Fig. 2.7). There is probably no benefit to be found to using the cyclotrino for ${ }^{40} \mathrm{~K}$, since conventional mass spectrometers are quite adequate for ${ }^{40} \mathrm{~K}$ measurements. There may be some applications for ${ }^{44} \mathrm{Ti}$ as a tracer, though its relatively short half life implies that the advantages of the cyclotrino over decay counting are not extremely large.

The most promising isotope other than ${ }^{14} \mathrm{C}$ seems to be ${ }^{26} \mathrm{Al}$. The abundance of ${ }^{26} \mathrm{Al}$ is about the same as that of ${ }^{14} \mathrm{C}$, so should pose no additional problems. The nearest interfering mass is different by a part in 3700 , so the cyclotron resolution would have to be doubled; this can be done by operating at about the 25th harmonic (Fig. 2.7). Furthermore, the abundance of the nearest interfering species $(25 \mathrm{MgH})$ should be relatively low. There will probably also be some mass-26 hydrocarbons produced (e.g. ${ }^{13} \mathrm{C}_{2}$, ${ }^{12} \mathrm{C}_{2} \mathrm{H}_{2}$ ), but these will be at least three times as far away in mass. Production of a beam of $\mathrm{Al}^{-}$ions is problematic; it may be necessary to use $\mathrm{AlO}^{-}$(Middleton, 1977). It seems that detection of ${ }^{26} \mathrm{Al}$ should be easily achieved if adequate ion beams can be produced. 


\section{CONCLUSIONS}

A small low energy cyclotron similar to that of Welch (1984a) has been combined with a high current external ion source, a beamline, and radial beam injection to detect ${ }^{14} \mathrm{C}$ at natural levels. The system's overall efficiency is about $2 \times 10^{-4}$. In its present form, it would be quite useful for measurements of samples enriched in ${ }^{14} \mathrm{C}$; it could improve the sensitivity of detecting ${ }^{14} \mathrm{C}$ in $1 \mu \mathrm{g}$ biomedical samples by four orders of magnitude over conventional scintillation counting techniques.

Improvements in system efficiency by a factor of 100 should be possible by redesigning the optics of the einzel lens, adding a beam buncher, and optimizing the detector geometry. With these modifications the system would be well suited to archaeological and geological applications.

The system could also be used for measurements of radioisotopes other than ${ }^{14} \mathrm{C}$. With minor modifications, ${ }^{26} \mathrm{Al}$ and ${ }^{3} \mathrm{He}$ should be detectable. With further modifications, ${ }^{10} \mathrm{Be}$ and other species may be measurable as well.

A small cyclotron such as this has great potential as a mass spectrometer. It offers a huge advantage over decay counting methods for small samples. With efficiency improvements, it would be able to do archaeological and geological measurements which are now done by tandem accelerators, yet would require a small fraction of the space, construction costs, and operating costs. 


\section{Appendix A: Some Isotopes of Potential Interest}

\begin{tabular}{|c|c|c|c|c|c|}
\hline Isotope & $\begin{array}{l}\text { Half } \\
\text { Life } \\
\text { (yrs) }\end{array}$ & $\begin{array}{l}\text { Stable } \\
\text { Isotope }\end{array}$ & $\begin{array}{l}\text { Natural } \\
\text { Abund. }\end{array}$ & $\begin{array}{l}\text { Stable } \\
\text { Isobar }\end{array}$ & $\begin{array}{c}\text { Mass } \\
\text { Diff. } \\
(\mathrm{m} / \Delta \mathrm{m})\end{array}$ \\
\hline${ }^{3} \mathrm{H}$ & 12.3 & $\begin{array}{c}{ }^{1} \mathrm{H} \\
{ }^{2} \mathrm{H}(.01 \%)\end{array}$ & $10^{-17}$ & $\begin{array}{c}{ }^{3} \mathrm{He}^{*} \\
\mathrm{HD} \\
\mathrm{H}_{3}\end{array}$ & $\begin{array}{c}-150,000 \\
+510 \\
+400\end{array}$ \\
\hline${ }^{10} \mathrm{Be}^{*}$ & $1.5 \mathrm{M}$ & ${ }^{9} \mathrm{Be}^{*}$ & $10^{-8}$ & $\begin{array}{c}{ }^{10} \mathrm{~B} \\
{ }^{9} \mathrm{BeH}\end{array}$ & $\begin{array}{c}-17,000 \\
+1500\end{array}$ \\
\hline${ }^{14} \mathrm{C}$ & 5730 & $\begin{array}{c}12 \mathrm{C} \\
{ }^{13} \mathrm{C}(1 \%)\end{array}$ & $10^{-12}$ & $\begin{array}{c}{ }^{14} \mathrm{~N}^{*} \\
{ }^{13} \mathrm{CH} \\
{ }^{12} \mathrm{CH}_{2}\end{array}$ & $\begin{array}{c}-83,000 \\
+1800 \\
+1100\end{array}$ \\
\hline${ }^{26} \mathrm{Al}$ & $705 \mathrm{k}$ & ${ }^{27} \mathrm{Al}$ & $10^{-14}$ & $\begin{array}{c}{ }^{26} \mathrm{Mg}^{*} \\
{ }^{25} \mathrm{MgH} \\
{ }^{13} \mathrm{C}_{2}\end{array}$ & $\begin{array}{l}-6000 \\
+3700 \\
+1300\end{array}$ \\
\hline${ }^{32} \mathrm{Si}$ & 100 & ${ }^{28} \mathrm{Si}$ & $10^{-16}$ & ${ }^{32} \mathrm{~S}$ & $-15,000$ \\
\hline${ }^{35} \mathrm{~S}$ & 87.2 days & $\begin{array}{c}{ }^{32} \mathrm{~S} \\
\text { also } 33,34,36\end{array}$ & & ${ }^{35} \mathrm{Cl}$ & $-48,000$ \\
\hline${ }^{36} \mathrm{Cl}$ & $300 \mathrm{k}$ & $\begin{array}{l}{ }^{35} \mathrm{Cl} \\
{ }^{37} \mathrm{Cl}\end{array}$ & $10^{-12}$ & $\begin{array}{c}{ }^{36} \mathrm{Ar}^{*} \\
{ }^{36} \mathrm{~S}\end{array}$ & $\begin{array}{l}-47,000 \\
-29,000\end{array}$ \\
\hline${ }^{39} \mathrm{Ar}^{*}$ & 269 & ${ }^{40} \mathrm{Ar}{ }^{*}$ & & ${ }^{39} \mathrm{~K}$ & $-64,000$ \\
\hline $40_{K}$ & $1.25 \mathrm{G}$ & ${ }^{39} \mathrm{~K}$ & $10^{-4}$ & $\begin{array}{l}{ }^{40} \mathrm{Ca}^{*} \\
{ }^{40} \mathrm{Ar}^{*} \\
{ }^{39} \mathrm{KH}\end{array}$ & $\begin{array}{c}-28,000 \\
-25,000 \\
+5700\end{array}$ \\
\hline${ }^{41} \mathrm{Ca}^{*}$ & $103 \mathrm{k}$ & ${ }^{40} \mathrm{Ca}^{*}$ & $10^{-12}$ & ${ }^{41} \mathrm{~K}$ & $-91,000$ \\
\hline${ }^{44} \mathrm{Ti}$ & 47 & $\begin{array}{c}{ }^{48} \mathrm{Ti} \\
\text { also } 46-50\end{array}$ & & $\begin{array}{l}{ }^{44} \mathrm{Ca}^{*} \\
{ }^{43} \mathrm{CaH}\end{array}$ & $\begin{array}{c}-10,000 \\
+4900\end{array}$ \\
\hline${ }^{53} \mathrm{Mn}$ & $3.7 \mathrm{M}$ & ${ }^{55} \mathrm{Mn}$ & $10^{-12}$ & ${ }^{53} \mathrm{Cr}$ & $-83,000$ \\
\hline${ }^{59} \mathrm{Ni}$ & $76 \mathrm{k}$ & $\begin{array}{c}{ }^{58} \mathrm{Ni} \\
\text { also } 60-62,64\end{array}$ & $10^{-12}$ & ${ }^{59} \mathrm{Co}$ & $-51,000$ \\
\hline${ }^{87} \mathrm{Rb}$ & $48.9 \mathrm{G}$ & ${ }^{85} \mathrm{Rb}$ & $28 \%$ & ${ }^{87} S r$ & $-287,000$ \\
\hline${ }^{129} \mathrm{I}$ & $15.7 \mathrm{M}$ & $127_{I}$ & $10^{-12}$ & $\begin{array}{l}{ }^{129} \mathrm{Xe}^{*} \\
{ }^{129} \mathrm{TeH} \\
{ }^{127} \mathrm{IH}_{2}\end{array}$ & $\begin{array}{c}-620,000 \\
+19,000 \\
+8800\end{array}$ \\
\hline
\end{tabular}

* Does nol form negative ion.

Data fiom Faure (1977), Middleton (1977), Muller et al. (1981), Nishiizumi and Amold (1981), Gencral Elrctric Co. (1984), Elmorc and Philips (1987) and Fink el al. (1987). 


\section{Appendix B: Evaluation of Integrals}

\section{B.1 Energy Gain}

The change in kinetic energy due to a gap crossing is approximated by (2.5):

$$
\delta T=\int F_{y} d y \equiv \int_{-\infty}^{\infty} q E_{y} d y
$$

The electric field $E_{y}$ (2.2) may be substituted to find:

$$
\begin{aligned}
\delta T & \cong \int_{-\infty}^{\infty} \frac{-q V_{\max }}{b} \frac{\cos \left(\frac{H y}{r_{c}}+\phi\right) \cos \left(\frac{\pi z}{b}\right) \cosh \left(\frac{\pi y}{b}\right)}{\cos ^{2}\left(\frac{\pi z}{b}\right)+\sinh ^{2}\left(\frac{\pi y}{b}\right)} d y \\
& \cong \frac{-q V_{\max }}{b} \cos \phi \cos \left(\frac{\pi z}{b}\right) \int_{-\infty}^{\infty} \frac{\cos \left(\frac{H y}{r_{c}}\right) \cosh \left(\frac{\pi y}{b}\right)}{\cos ^{2}\left(\frac{\pi z}{b}\right)+\sinh ^{2}\left(\frac{\pi y}{b}\right)} d y
\end{aligned}
$$

As mentioned previously, the integral extending to infinity assumes that $b \ll r$. The use of a constant $r$ in the cos factor is based on the assumption that $\delta T \ll T$.

This integral appears somewhat foreboding, but may be simplified and expanded with partial fractions:

$$
\begin{gathered}
\int_{-\infty}^{\infty} \frac{\cos \left(\frac{H y}{r_{c}}\right) \cosh \left(\frac{\pi y}{b}\right)}{\cos ^{2}\left(\frac{\pi z}{b}\right)+\sinh ^{2}\left(\frac{\pi y}{b}\right)} d y=\int_{-\infty}^{\infty} \frac{\cos \left(\frac{H y}{r_{c}}\right) \cosh \left(\frac{\pi y}{b}\right)}{\cosh ^{2}\left(\frac{\pi y}{b}\right)-\sin ^{2}\left(\frac{\pi z}{b}\right)} d y \\
=\int_{0}^{\infty} \frac{\cos \left(\frac{H y}{r_{c}}\right)}{\cosh \left(\frac{\pi y}{b}\right)-\sin \left(\frac{\pi z}{b}\right)} d y+\int_{0}^{\infty} \frac{\cos \left(\frac{H y}{r_{c}}\right)}{\cosh \left(\frac{\pi y}{b}\right)+\sin \left(\frac{\pi z}{b}\right)} d y
\end{gathered}
$$


These terms may be integrated analytically (Gradshteyn and Ryzhik, 1980, 3.983 $\# 1)$ :

$$
\int_{0}^{\infty} \frac{\cos \left(\frac{H y}{r_{c}}\right)}{\cosh \left(\frac{\pi y}{b}\right) \pm \sin \left(\frac{\pi z}{b}\right)} d y=\frac{b \sinh \left(\frac{H b}{2 r_{c}} \mp \frac{H z}{r_{c}}\right)}{\cos \left(\frac{\pi z}{b}\right) \sinh \left(\frac{H b}{r_{c}}\right)}
$$

Substitution into (B.2) results in:

$$
\delta T \cong-q V_{\max } \cos \phi\left(\frac{\sinh \left(\frac{H b}{2 r_{c}}+\frac{H z}{r_{c}}\right)+\sinh \left(\frac{H b}{2 r_{c}}-\frac{H z}{r_{c}}\right)}{\sinh \left(\frac{H b}{r_{c}}\right)}\right)
$$

which reduces to $(2.6)$ :

$$
\delta T \cong-q V_{m} \cos \phi\left[\frac{\cosh \frac{H z}{r}}{\cosh \frac{H b}{2 r}}\right]
$$

\section{B.2 Axial Focusing}

This integral may be solved similarly to the integral for energy gain discussed above.

The impulse which an ion receives at a gap crossing is given by (2.9):

$$
\begin{aligned}
\delta p_{z} & \cong \int_{t=-\infty}^{t=\infty} q E_{z} d t \\
& \cong q \int_{y=-\infty}^{y=\infty} \frac{E_{z}(y)}{V_{y}(y)} d y
\end{aligned}
$$

Substitution of $E_{z}$ (2.3) results in: 


$$
\delta p_{z} \cong \int_{-\infty}^{\infty} \frac{-q V_{\max }}{b V_{y}} \frac{\cos \left(\frac{H y}{r_{c}}+\phi\right) \sin \left(\frac{\pi z}{b}\right) \sinh \left(\frac{\pi y}{b}\right)}{\cos ^{2}\left(\frac{\pi z}{b}\right)+\sinh ^{2}\left(\frac{\pi y}{b}\right)} d y
$$

where, again, the approximations that that $b \ll r$ and $\delta T \ll T$ are implicit. For $\delta T \ll T$, velocity is roughly constant across the gap and $v(y)$ may be pulled out of the integral. Thus:

$$
\delta p_{z} \cong \frac{-q V_{\max } \sin \phi \sin \left(\frac{H y}{r_{c}}\right)}{b V_{y}} \int_{-\infty}^{\infty} \frac{\sin \left(\frac{\pi z}{b}\right) \sinh \left(\frac{\pi y}{b}\right)}{\cos ^{2}\left(\frac{\pi z}{b}\right)+\sinh ^{2}\left(\frac{\pi y}{b}\right)} d y
$$

The integral may be simplified by a partial fraction expansion as in section B.1:

$$
\begin{gathered}
\int_{-\infty}^{\infty} \frac{\sin \left(\frac{H y}{r_{c}}\right) \sinh \left(\frac{\pi y}{b}\right)}{\cos ^{2}\left(\frac{\pi z}{b}\right)+\sinh ^{2}\left(\frac{\pi y}{b}\right)} d y=\int_{-\infty}^{\infty} \frac{\sin \left(\frac{H y}{r_{c}}\right) \sinh \left(\frac{\pi y}{b}\right)}{\cosh ^{2}\left(\frac{\pi y}{b}\right)-\sin ^{2}\left(\frac{\pi z}{b}\right)} d y \\
=\frac{1}{\sin \left(\frac{\pi z}{b}\right)}\left\{\int_{0}^{\infty} \frac{\sin \left(\frac{H y}{r_{c}}\right) \sinh \left(\frac{\pi y}{b}\right)}{\cosh \left(\frac{\pi y}{b}\right)-\sin \left(\frac{\pi z}{b}\right)} d y-\int_{0}^{\infty} \frac{\sin \left(\frac{H y}{r_{c}}\right) \sinh \left(\frac{\pi y}{b}\right)}{\cosh \left(\frac{\pi y}{b}\right)+\sin \left(\frac{\pi z}{b}\right)} d y\right\}
\end{gathered}
$$

Again, the terms may be integrated analytically (Gradshteyn and Ryzhik, 1980, 3.984 \#1):

$$
\int_{0}^{\infty} \frac{\sin \left(\frac{H y}{r_{c}}\right) \sinh \left(\frac{\pi y}{b}\right)}{\cosh \left(\frac{\pi y}{b}\right) \pm \sin \left(\frac{\pi z}{b}\right)} d y=\frac{b \cosh \left(\frac{H b}{2 r_{c}} \mp \frac{H z}{r_{.}}\right)}{\sinh \left(\frac{H b}{r_{c}}\right)}
$$

Substitution into (B.8) results in: 


$$
\delta p_{z} \cong \frac{q V_{\max } \sin \phi}{v_{y}}\left(\frac{\cosh \left(\frac{H b}{2 r_{c}}+\frac{H z}{r_{c}}\right)-\cosh \left(\frac{H b}{2 r_{c}}-\frac{H z}{r_{c}}\right)}{\sinh \left(\frac{H b}{r_{c}}\right)}\right)
$$

which reduces to $(2.10)$ :

$$
\delta p_{z} \equiv \frac{q V_{m} \sin \phi}{V}\left[\frac{\sinh \frac{H z}{r}}{\cosh \frac{H b}{2 r}}\right]
$$

For an elegant alternative derivation of this result and the previous see Jeffries (1951). He forms an analytic function from the two integrals and solves them simultaneously by contour integration.

\section{B.3 Phase Bunching}

As stated in the text (section 2.3.3), the phase change may be calculated by integrating the angle subtended as an ion crosses the gap region and subtracting the total angle which would have been subtended if the energy gain had all occurred at an infinitesimal-width gap. The subtended angle may be approximated as $d \phi \cong d y / r$, thus:

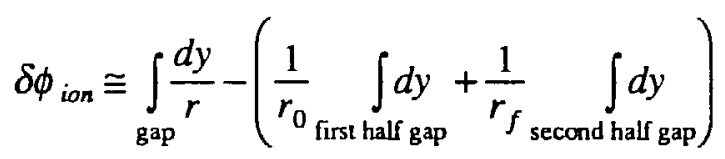

Assuming that $\delta T \ll T$, and that $b \ll r$ (since the distance over which the electric field extends is on the order of $b$ ), the phase change experienced in a single gap crossing may be approximated as:

$$
\delta \phi_{\text {ion }} \equiv \int_{-\infty}^{0}\left(\frac{1}{r(y)}-\frac{1}{r_{-\infty}}\right) d y+\int_{0}^{\infty}\left(\frac{1}{r(y)}-\frac{1}{r_{\infty}}\right) d y
$$

We may write $r(y)=r_{-\infty}+d r_{1}(y)$ for the first half of the dee gap, and $r(y)=r_{\infty}+$ $d r_{2}(y)$ for the second half. Then, assuming $\delta T \ll T$ (thus $d r \ll r$ ), the equation may be written as: 


$$
\begin{aligned}
\delta \phi_{\text {ion }} & \cong \frac{-1}{r_{-\infty}^{2}} \int_{-\infty}^{0} d r_{1}(y) d y+\frac{-1}{r^{2}} \int_{0}^{\infty} d r_{2}(y) d y \\
& \cong \frac{-1}{2 r_{-\infty} T_{-\infty}} \int_{-\infty}^{0} d T_{1}(y) d y+\frac{-1}{2 r_{\infty} T_{\infty}} \int_{0}^{\infty} d T_{2}(y) d y
\end{aligned}
$$

Now, $d T_{1}(y)=\int_{-\infty}^{y} F_{y}(\eta) d \eta$, and $d T_{2}(y)=\int_{\infty}^{y} F_{y}(\eta) d \eta$. These integrals may be evaluated by parts:

$$
\int_{-\infty}^{0} \int_{-\infty}^{y} F_{y}(\eta) d \eta u^{l}=-\int_{-\infty}^{0} y F_{y}(y) d y
$$

To first order, $r$ and $T$ are constants, so the two integrals in (B.14) may be combined. Thus:

$$
\delta \phi_{\text {ion }} \equiv \frac{1}{2 r T} \int_{y=-\infty}^{y=\infty} y d T
$$

However, this phase is in terms of the ion's cyclotron frequency. The phase with respect to the RF waveform is the crucial parameter, and since the RF may be operated at a harmonic $H$, the phase shift with respect to the RF will be greater by a factor of $H$, yielding (2.19):

$$
\begin{aligned}
\delta \phi & \cong \frac{H}{2 r T} \int_{y=-\infty}^{y=\infty} y d T \cong \frac{H}{2 r T} \int_{-\infty}^{\infty} y F_{y}(y) d y \\
& \equiv \frac{q H}{2 r T} \int_{-\infty}^{\infty} y E_{y}(y) d y
\end{aligned}
$$

where $\phi_{\text {ion }}=$ ion phase and $\phi=\mathrm{RF}$ phase $=H \phi_{\text {ion }}$.

Substituting for $E_{y}$ : 


$$
\begin{aligned}
\delta \phi & \cong \frac{-q H V_{\max }}{2 b r T} \int_{-\infty}^{\infty} \frac{\cos \left(\frac{H y}{r_{c}}+\phi\right) \cos \left(\frac{\pi z}{b}\right) \cosh \left(\frac{\pi y}{b}\right)}{\cos ^{2}\left(\frac{\pi z}{b}\right)+\sinh ^{2}\left(\frac{\pi y}{b}\right)} y d y \\
& \cong \frac{q H V_{\max } \sin \phi \cos \left(\frac{\pi z}{b}\right)}{2 b r T} \int_{-\infty}^{\infty} \frac{y \sin \left(\frac{H y}{r_{c}}\right) \cosh \left(\frac{\pi y}{b}\right)}{\cos ^{2}\left(\frac{\pi z}{b}\right)+\sinh ^{2}\left(\frac{\pi y}{b}\right)} d y
\end{aligned}
$$

Again, the integral may be simplified by expansion in partial fractions:

$$
\begin{gathered}
\int_{-\infty}^{\infty} \frac{y \sin \left(\frac{H y}{r_{c}}\right) \cosh \left(\frac{\pi y}{b}\right)}{\cos ^{2}\left(\frac{\pi z}{b}\right)+\sinh ^{2}\left(\frac{\pi y}{b}\right)} d y=\int_{-\infty}^{\infty} \frac{y \sin \left(\frac{H y}{r_{c}}\right) \cosh \left(\frac{\pi y}{b}\right)}{\cosh ^{2}\left(\frac{\pi y}{b}\right)-\sin ^{2}\left(\frac{\pi z}{b}\right)} d y \\
=\int_{0}^{\infty} \frac{y \sin \left(\frac{H y}{r_{c}}\right)}{\cosh \left(\frac{\pi y}{b}\right)-\sin \left(\frac{\pi z}{b}\right)} d y+\int_{0}^{\infty} \frac{y \sin \left(\frac{H y}{r_{c}}\right)}{\cosh \left(\frac{\pi y}{b}\right)+\sin \left(\frac{\pi z}{b}\right)} d y
\end{gathered}
$$

These terms may be integrated analytically. One may find (taking a limit of Gradshteyn and Ryzhik, 1980, 3.983 \#5):

$$
\begin{aligned}
& \int_{0}^{\infty} \frac{y \sin a y}{\cosh \gamma y-\cos \delta} d y= \\
& =\frac{\pi}{\gamma^{2} \sin \delta \sinh ^{2}\left(\frac{\pi a}{\gamma}\right)}\left(\pi \cosh \left(\frac{\pi a}{\gamma}\right) \sinh \left(\frac{\delta a}{\gamma}\right)-\delta \sinh \left(\frac{\pi a}{\gamma}\right) \cosh \left(\frac{\delta a}{\gamma}\right)\right)
\end{aligned}
$$

Changing variables, we find:

$$
\int_{0}^{\infty} \frac{y \sin \left(\frac{H y}{r_{c}}\right)}{\cosh \left(\frac{\pi y}{b}\right) \pm \sin \left(\frac{\pi z}{b}\right)} d y=\frac{b^{2}(A+B)}{4 \pi \cos \left(\frac{\pi z}{b}\right) \sinh ^{2}\left(\frac{H b}{2 r}\right) \cosh ^{2}\left(\frac{H b}{2 r}\right)}
$$


where:

$$
\begin{aligned}
& A=\cosh \left(\frac{H z}{r}\right) \sinh \left(\frac{H b}{2 r}\right)\left(\pi \sinh ^{2}\left(\frac{H b}{2 r}\right) \pm \frac{2 \pi z}{b} \cosh ^{2}\left(\frac{H b}{2 r}\right)\right) \\
& B=\sinh \left(\frac{H z}{r}\right) \cosh \left(\frac{H b}{2 r}\right)\left(\frac{2 \pi z}{b} \sinh ^{2}\left(\frac{H b}{2 r}\right) \mp \pi \cosh ^{2}\left(\frac{H b}{2 r}\right)\right)
\end{aligned}
$$

Substituting and simplifying:

$$
\begin{aligned}
& \int_{-\infty}^{\infty} \frac{y \sin \left(\frac{H y}{r_{c}}\right) \cosh \left(\frac{\pi y}{b}\right)}{\cosh ^{2}\left(\frac{\pi y}{b}\right)-\sin ^{2}\left(\frac{\pi z}{b}\right)} d y= \\
& =\frac{b^{2}}{2 \cos \frac{\pi z}{b}}\left(\frac{\cosh \left(\frac{H z}{r}\right) \sinh \left(\frac{H b}{2 r}\right)+\frac{2 z}{b} \sinh \left(\frac{H z}{r}\right) \cosh \left(\frac{H b}{2 r}\right)}{\cosh ^{2}\left(\frac{H b}{2 r}\right)}\right)
\end{aligned}
$$

Thus:

$$
\delta \phi \cong \frac{q V_{m} H b}{4 r T} \sin \phi\left(\frac{\cosh \left(\frac{H z}{r}\right) \sinh \left(\frac{H b}{2 r}\right)+\frac{2 z}{b} \sinh \left(\frac{H z}{r}\right) \cosh \left(\frac{H b}{2 r}\right)}{\cosh ^{2}\left(\frac{H b}{2 r}\right)}\right)
$$

This expression is based on the approximations that $\delta T \ll T$ and $b \ll r$, and that the gap between dees is infinitesimal. 


\section{Appendix C: Electronics}

A number of custom circuits have been designed and built for the small cyclotron. These will be described in this appendix.

\section{C.1 RF Circuitry}

The dees must be driven with a high voltage at typically 9 to $15 \mathrm{MHz}$. We have used a commercial amateur radio transceiver (Kenwood 930S) with an oven-controlled crystal which has been modified so that it will transmit at any frequency between 1.5 and $30 \mathrm{Mhz}$. This feeds a wideband linear amplifier (ENI 3200L) which provides up to 200 watts output and is very tolerant of impedance mismatches. The $50 \mathrm{ohm}$ output impedance of the amplifier must be matched to the cyclotron. The cyclotron is essentially a capacitor to ground of about $140 \mathrm{pF}$, with a large parallel resistance representing the power lost due to resistance, eddy currents, and energy gain of ions.

This matching is performed with a standard matching network, as shown in Fig. C.1. The inductance and capacitance are varied to match the load to $50 \mathrm{ohms}$.

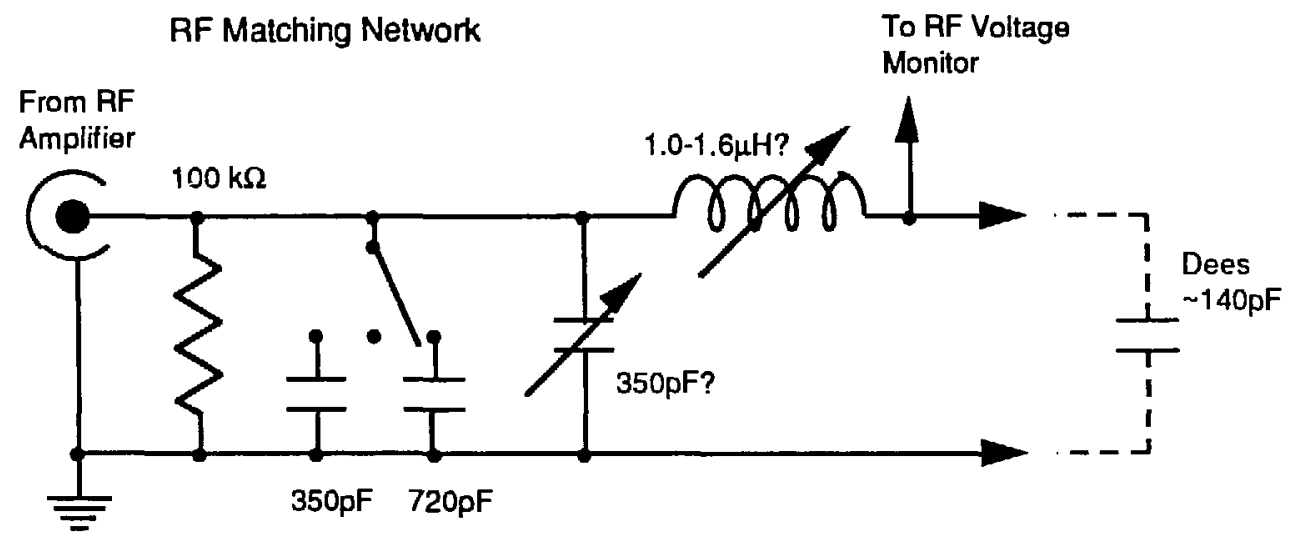

Figure C.1 RF matching network.

A circuit was built to monitor the voltage on the dees (Fig. C.2). The dee voltage is divided by 100 in a capacitive voltage divider. (A resistive divider would either have too much power loss or too high impedance to function properly.) This signal is then sent through a peak detector circuit and buffered by an op-amp. The diode D1 is a hot-carrier type, since it must respond at manv $\mathrm{MHz}$. The diode drop is cancelled by placing an 
identical diode, D2, in the op-amp's feedback loop, operating with the same DC current. Resistor R1 is necessary to provide a DC current path for the peak detector. The buffered voltage and a ground reference are sent through a cable to a rack-mounted digital panel meter in a chassis which also contains circuitry for the Hall probe (Fig. C.3). A potentiometer on the input of the meter calibrates the reading.

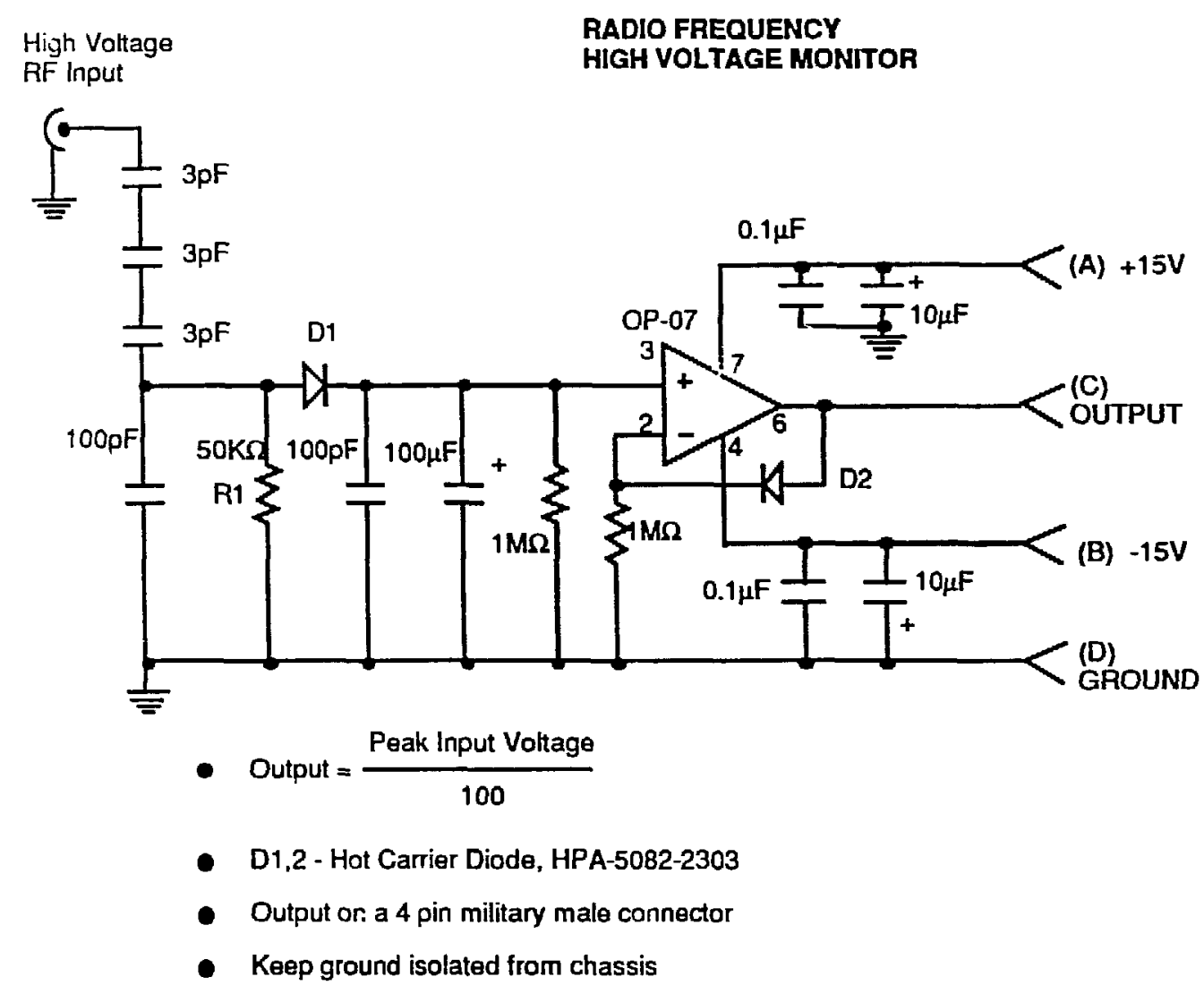

Figure C.2 Dee voltage monitoring circuitry.

We found that this circuit had a severe, unexplained response notch at about 10 $\mathrm{MHz}$. This is presumably due to parasitic reactances. Rather than redesign the circuit, a curve was generated providing a multiplying factor to correct the readings. 

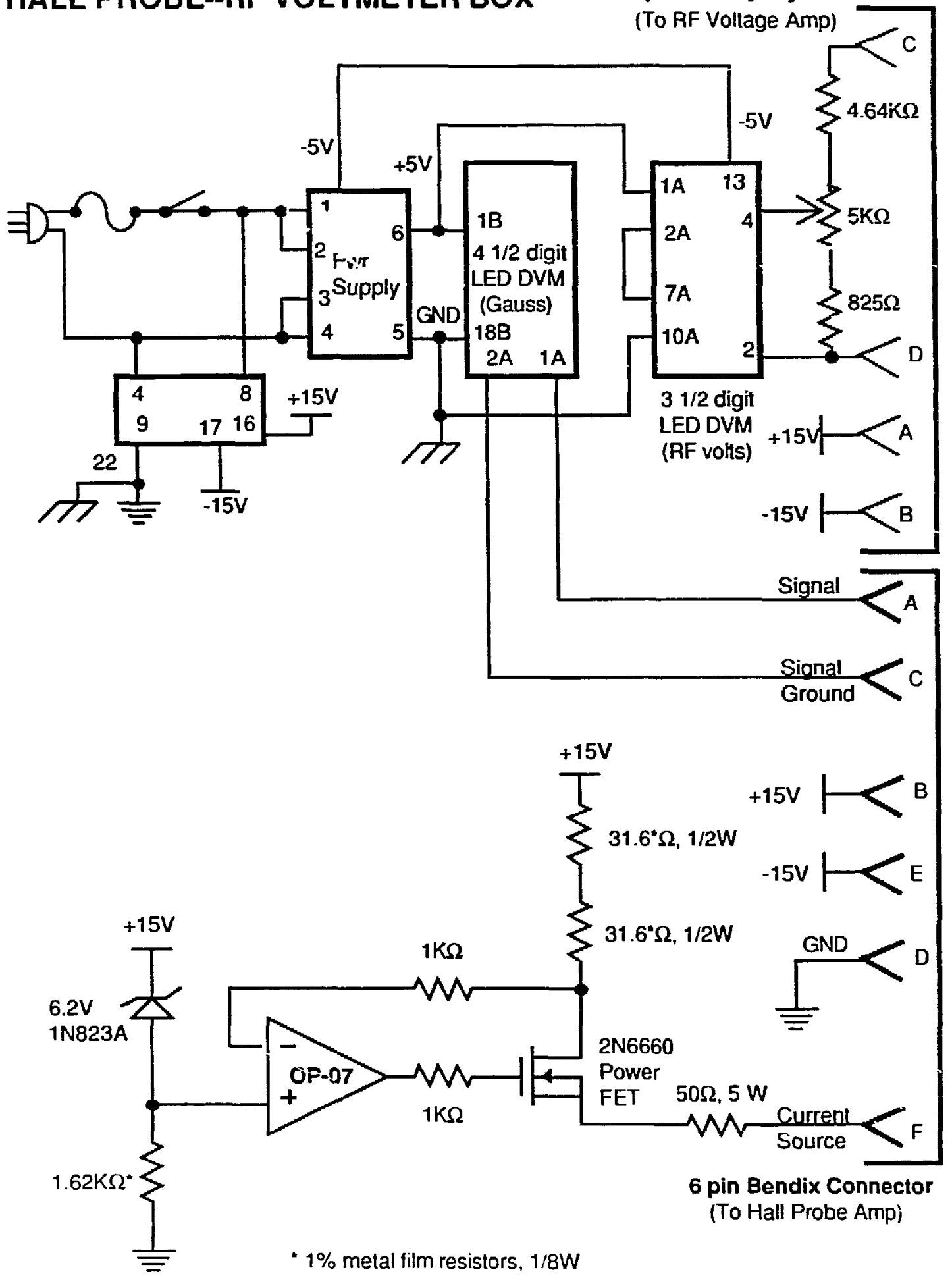

Figure C.3 Hall probe/RF voltmeter display unit. 


\section{C.2 Magnetic Field Measurement}

A circuit was built to measure the magnetic field strength inside the cyclotron. This uses a high linearity Hall effect device mounted near the center of the cyclotron. An instrumentation amplifier circuit is located near the vacuum chamber (Fig. C.4). The preamplified signal is then sent to a DVM in a rack-mount chassis (Fig. C.3), which also contains the constant current source for the Hall device and the panel meter for the RF voltage measurement circuit of Fig. C.2.

\section{GAUSSMETER}
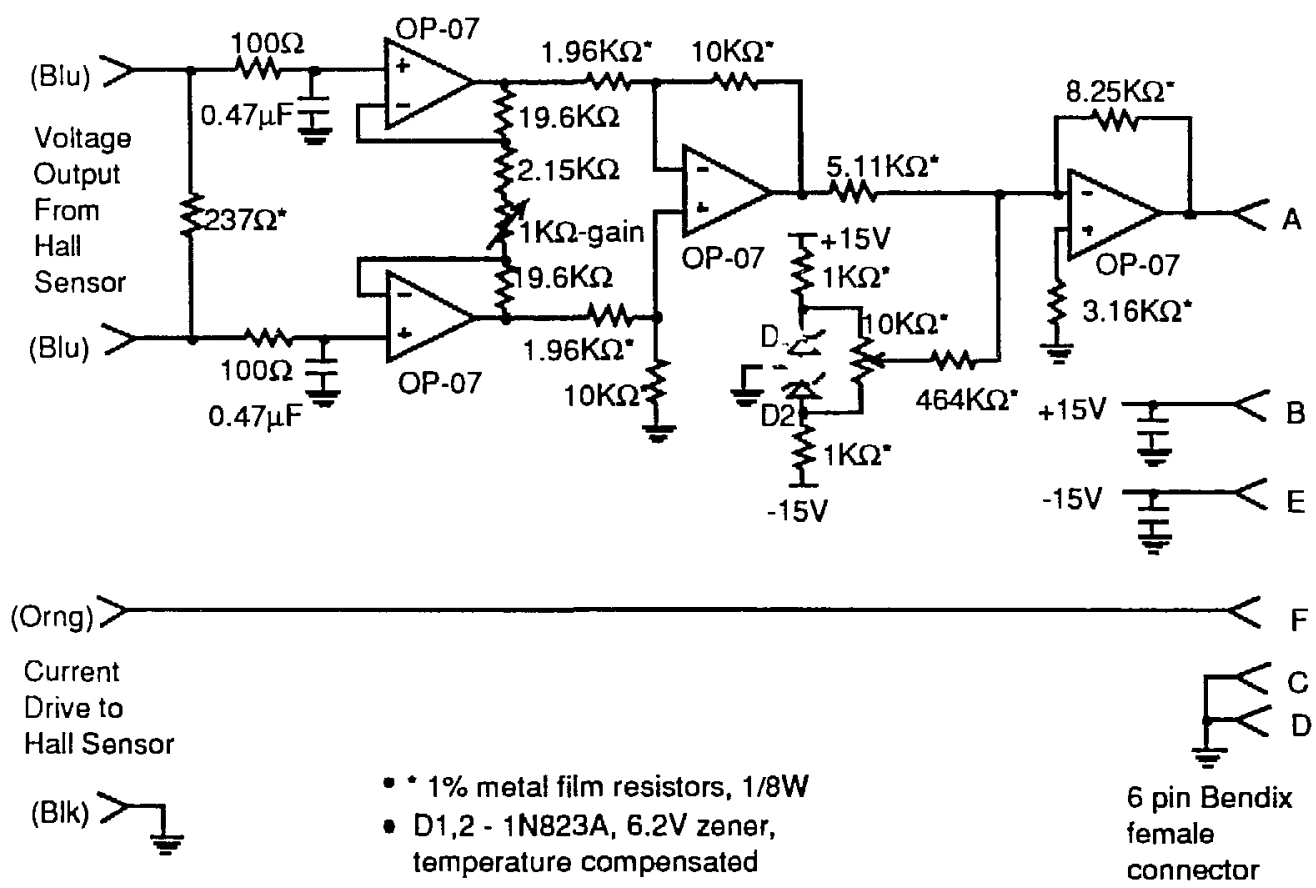

Figure C.4 Hall probe amplifier.

\section{C.3 High Voltage Circuitry}

A number of high voltage supplies are used in the small cyclotron; none exceeds $5 \mathrm{kV}$. A number of connectors are used for these supplies, including MHV, SHV, and $10 \mathrm{kV}$ Reynolds. High voltage feedthroughs into the vacuum erclosire are via MHV 
connectors; these are used because they were present on pre-existing hardware. They will mate with BNC connectors. This can be advantageous in that it allows the high voltage to be disconnected from a device and the current intercepted by the device to be monitored instead. But MHV connectors pose a safety hazard. Although they are rated at $5 \mathrm{kV}$, if energized with $5 \mathrm{kV}$ while not connected, a spark is easily drawn from the end of the connector due to the geometry of the electrodes. The system was made safe by building a number of small current limiting boxes.

The circuit of these protection boxes is shown in Fig. C.5. Resistor R1 safely limits current at $5 \mathrm{kV}$ to $50 \mu \mathrm{A}$. Capacitor $\mathrm{C} 1$ filters any ripple which may otherwise appear on the line. The neon light indicates current flow, providing an indication of either arcing or conduction in the vacuum enclosure. Resistor R2 is necessary to eliminate voltage drop across the neon light when no current is flowing.

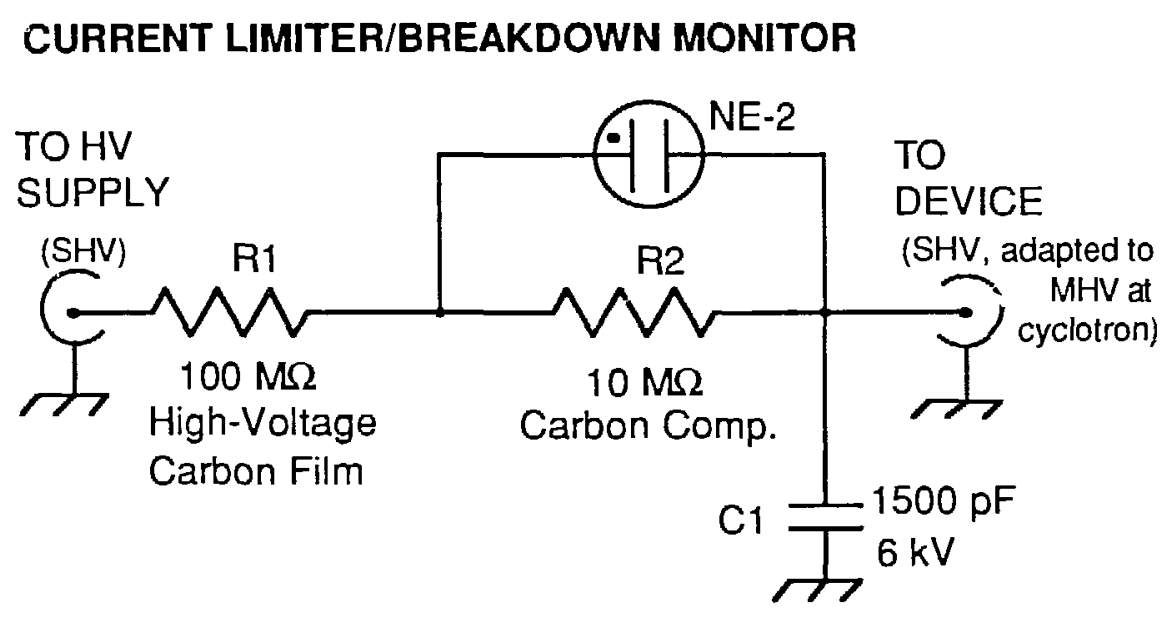

Figure C.5 Circuit for limiting current and indicating arcing of high voltage electrostatic devices.

The Wien filter is connected somewhat differently. Since it may collect significant current (more than a milliamp), a series resistor is problematic. Thus, a clamp has been placed over the dangerous MHV connectors to prohibit their removal, and a box with a high voltage switch has been assembled to enable current measurements (Fig. C.6). This box also contains a resistor divider which provides the potential for the guard electrode in the Wien filter. 


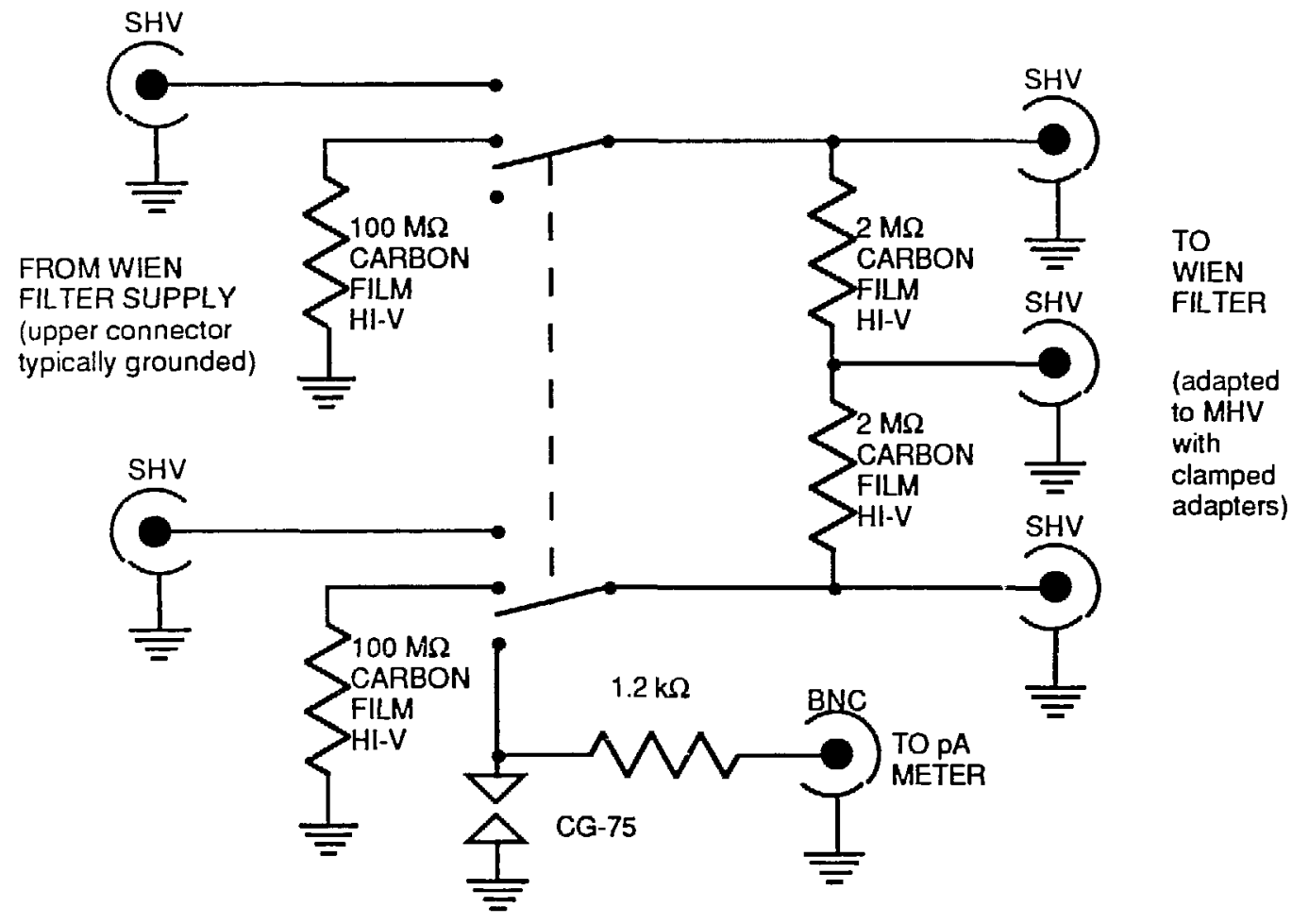

Figure C.6 Switchbox for Wien filter electrodes.

The microchannel plate detector requires a number of voltages for its functioning. A circuit was built to properly distribute the voltages, to couple the output pulses to a preamplifier, and to protect the microchannel plate. This is shown in Fig. C.7. The detector's dynode is at ground potential. Negative high voltage leads go to the microchannel plate's input, output, and anode. High value resistors provide some protection for the channel plate. Further protection is provided by a string of neon bulbs; this limits the maximum voltage which may be applied across the channel plate. Output current pulses from the anode are coupled to a preamplifier at ground potential through a capacitor. 

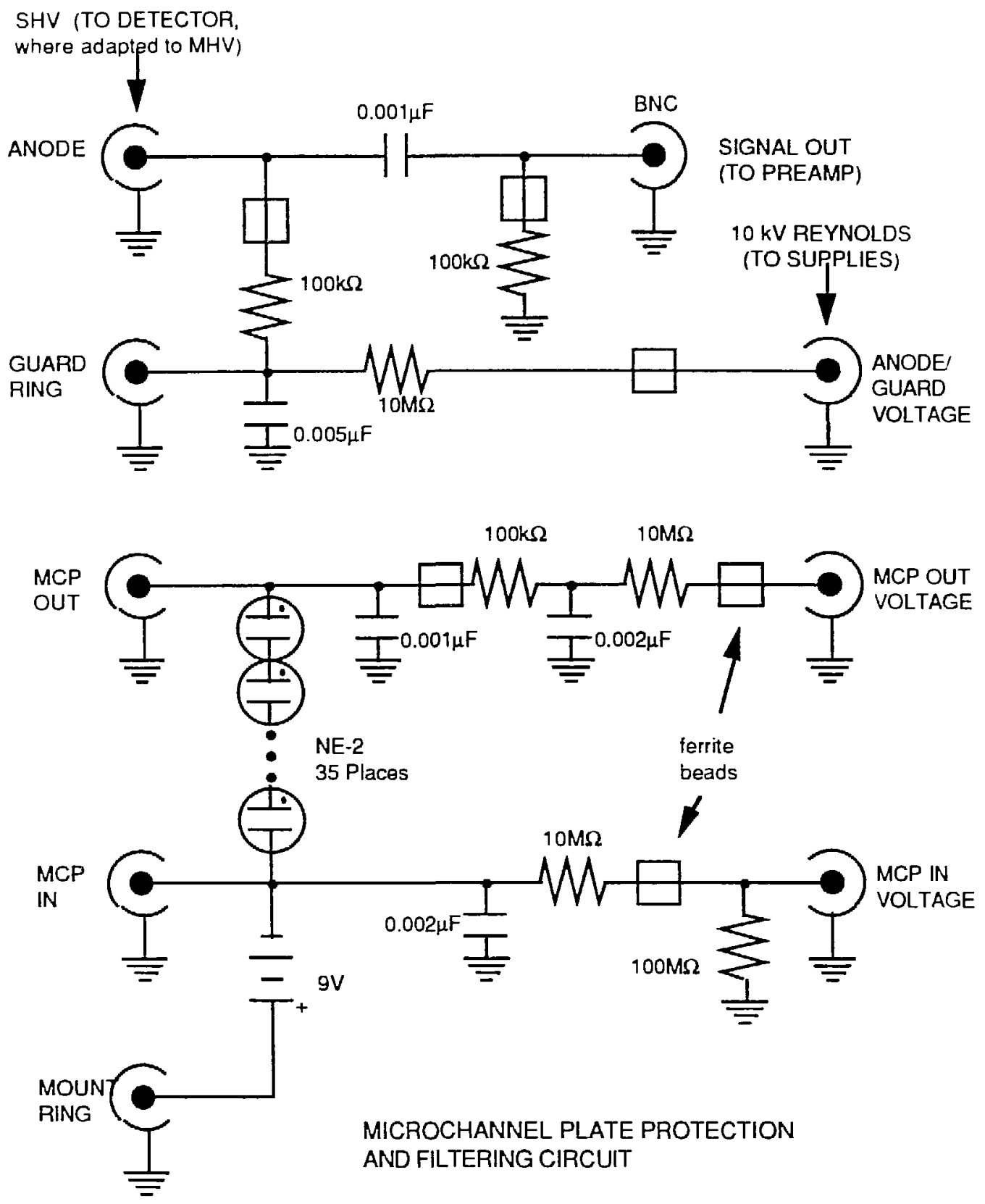

Figure C.7 Circuitry to protect microchannel plate detector and to filter and distribute voltages. 


\section{C.4 Power Supplies}

A power supply was designed and built to run the negative ion source. The source requires a heater current of about 2.5 amps at about 20 volts and an ionizing current of about 20 amps at 12 volts. The circuitry designed is shown in Fig. C.8. The supplies are isolated from ground so that the ion source may be biased as much as $2 \mathrm{kV}$ from ground. Interlocks are provided so that the cesium heater may not be energized unless the ionizer is energized, and the ionizer may not be turned on unless the carbon target high voltage is present. The entire supply is also interlocked with the ion source's Freon cooling loop, so that operation is not possible unless Freon is flowing. Analog meters show voltages and currents, and meters also display cesium energy and cesium heater temperature. (The heater temperature measuring circuitry does not operate properly; this is apparently due to grounding problems, either ground loops or noise. Ensuring that the thermocouple is electrically isolated from ground would probably help.) 


\section{Ion Source Power Supply (page 1 of 2)}
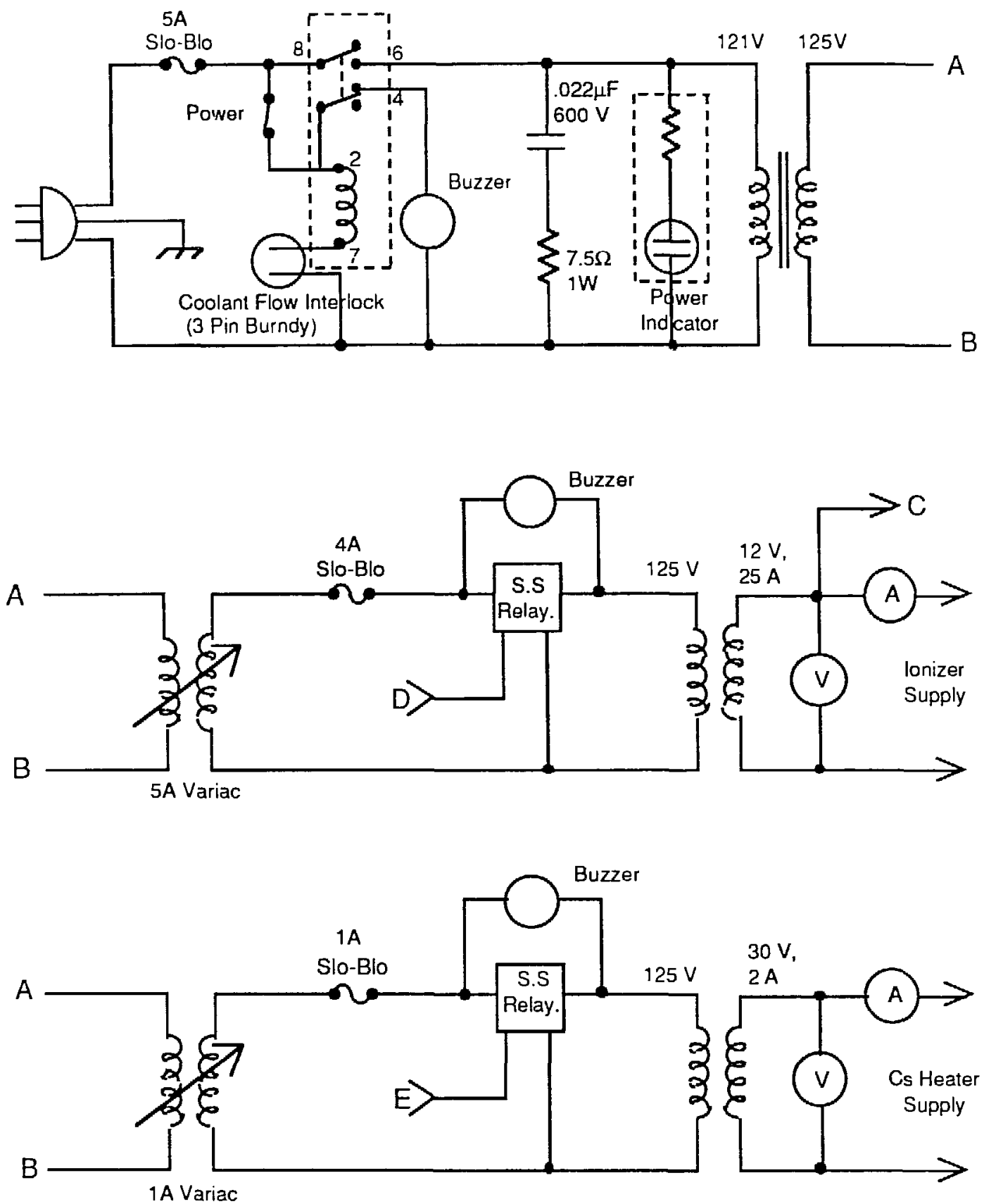

Figure C.8a Power supply generating AC voltages for ion source. 

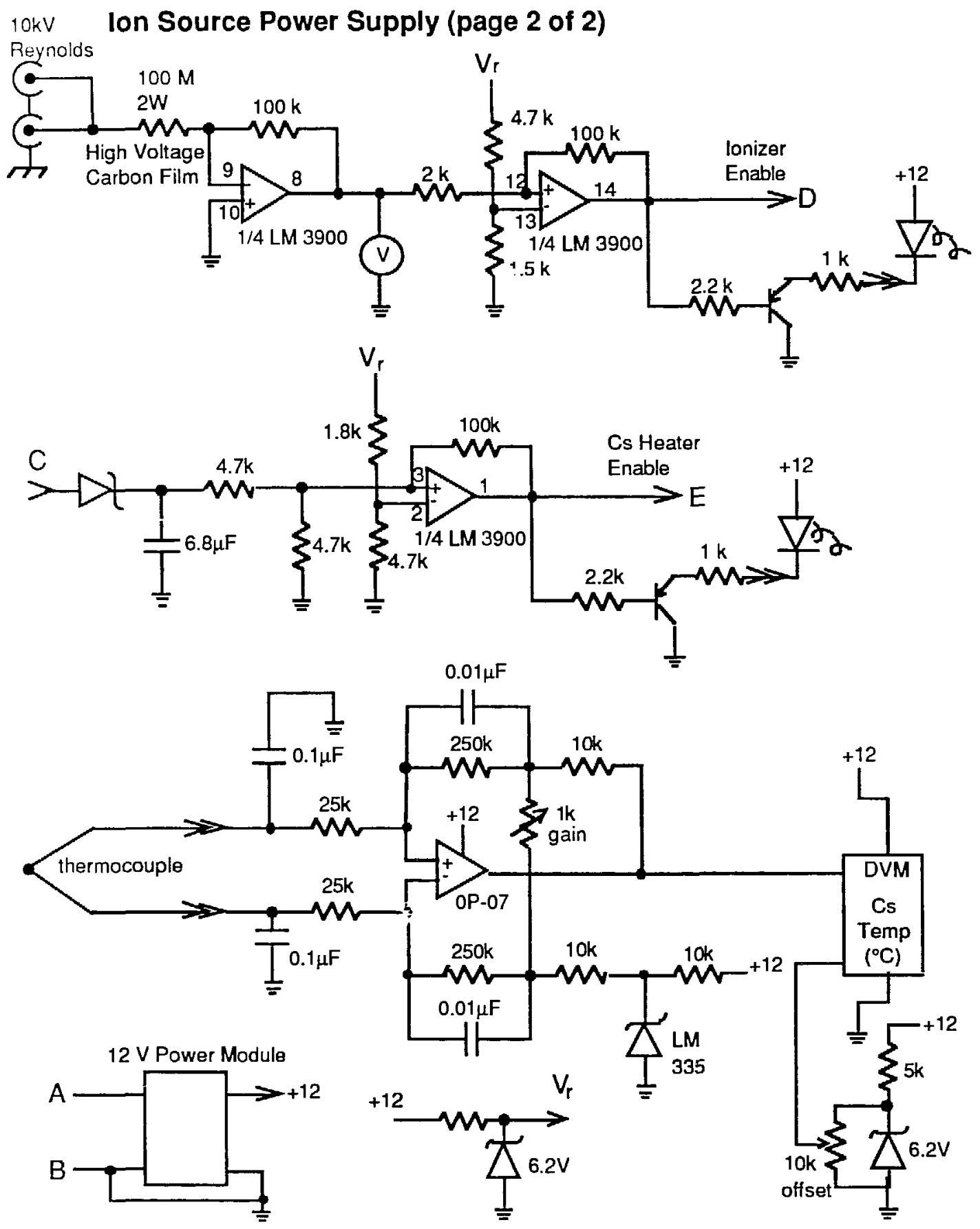

Figure C.8b Interlocks and thermocouple measurement circuit for ion source.

Power supplies were also designed and built to drive the quadrupole lenses. These supplies are fairly complex (Fig. C.9). Each quadrupole element requires a DC voltage of \pm 500 volts or less with negligible current, and has a capacitance to ground of 10 to 1000 
$\mathrm{pF}$, primarily due to wiring rather than to the structures themselves. A command voltage between -5 and +5 volts is generated for each of the quadrupole elements by linearly combining focus, deflection, and mean energy settings in op-amp circuitry. Each quadrupole element's output voltage is divided by 100 and subtracted from the command voltage. The resultant error voltage is forced to zero in a feedback loop.

\section{QUADRAPOLE SUPPLY POWER SUPPLY BOARD:}
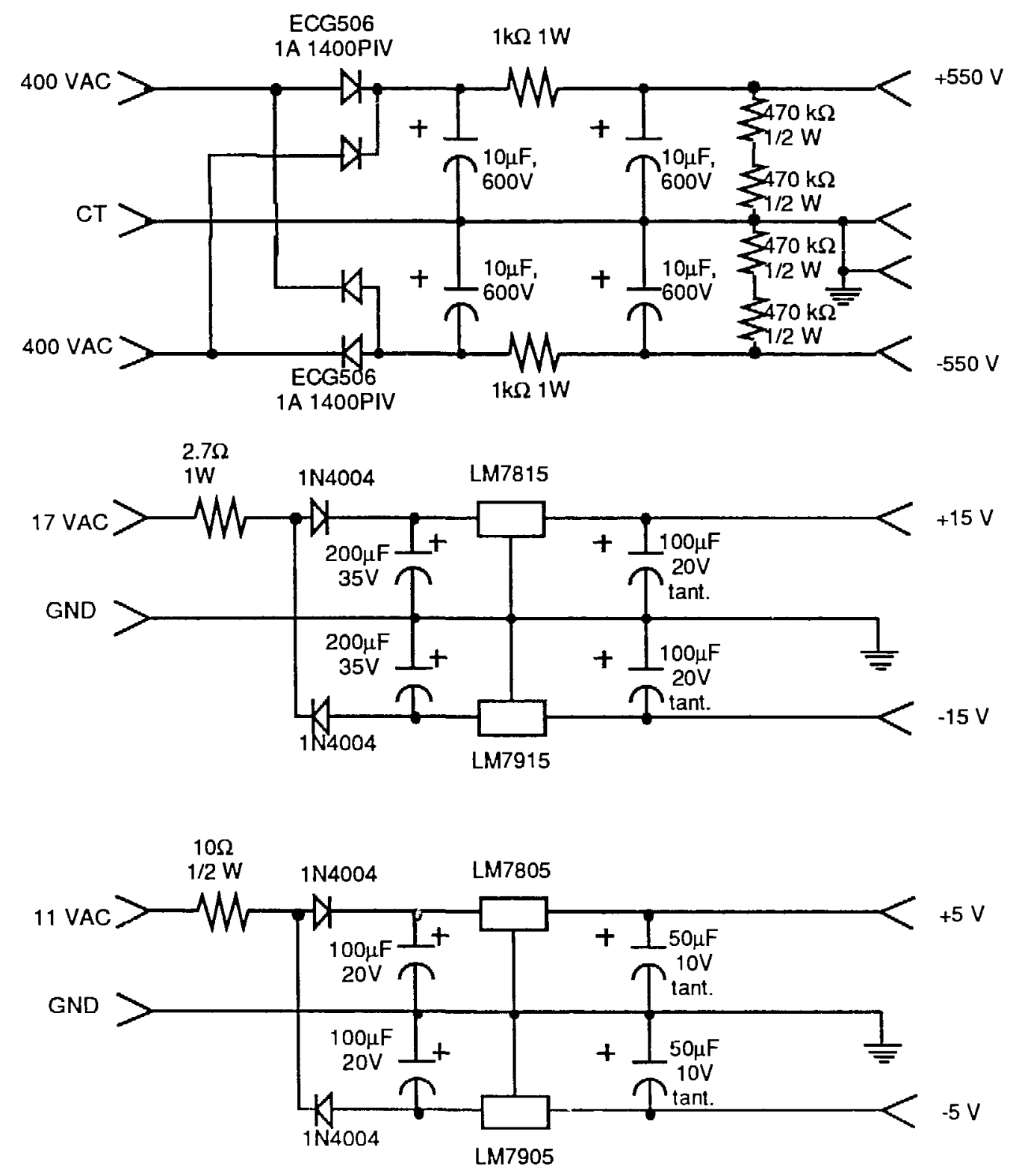

Figure C.9a Supply section of quadrupole power supply. 

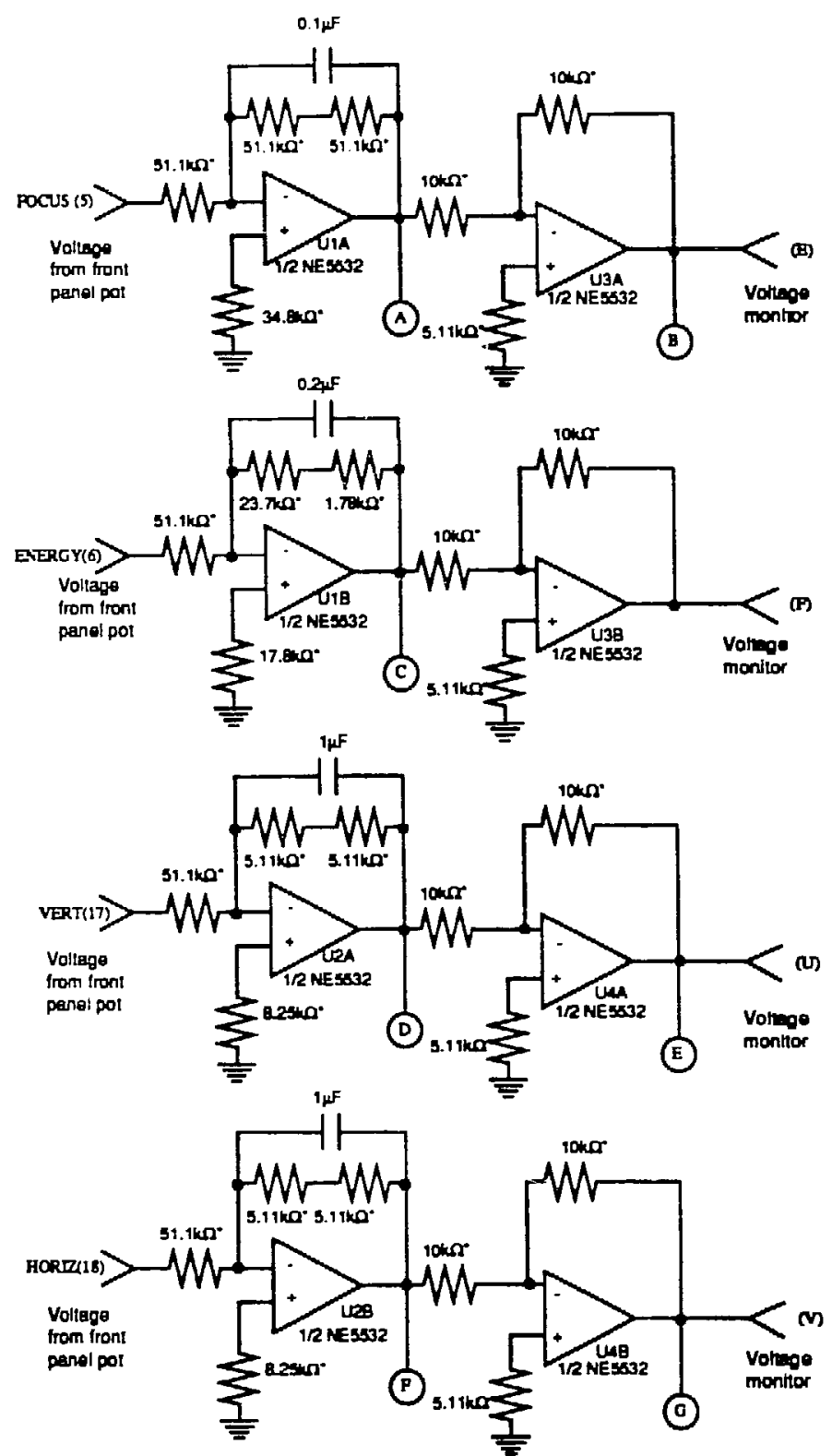

QUADRAPOLE SUPPLY INPUT BOARD:

Noles:

1. Add $0.1 \mu \mathrm{F}$ cap on each apply of each

l.C.

2 One of four rections shown

3. Edge connector (44 pin) pinouts are given in perentheses

4. " deotes 1\% 1/8 W manl film rairtors

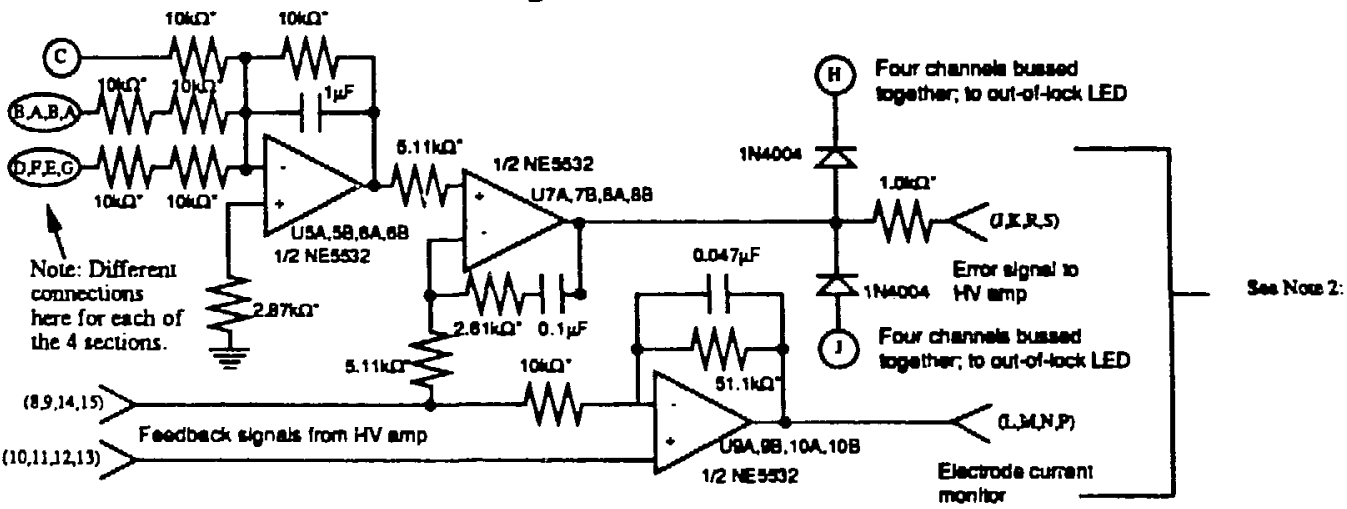

Figure C.9b Lo:s voltage input board of quadrupole power supply.

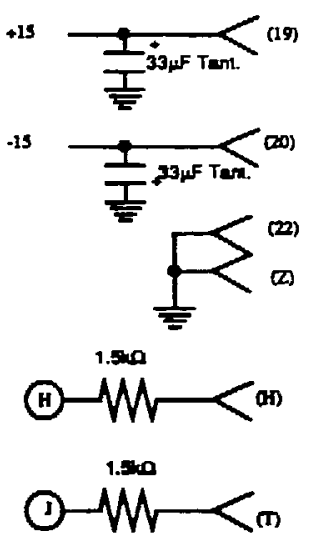




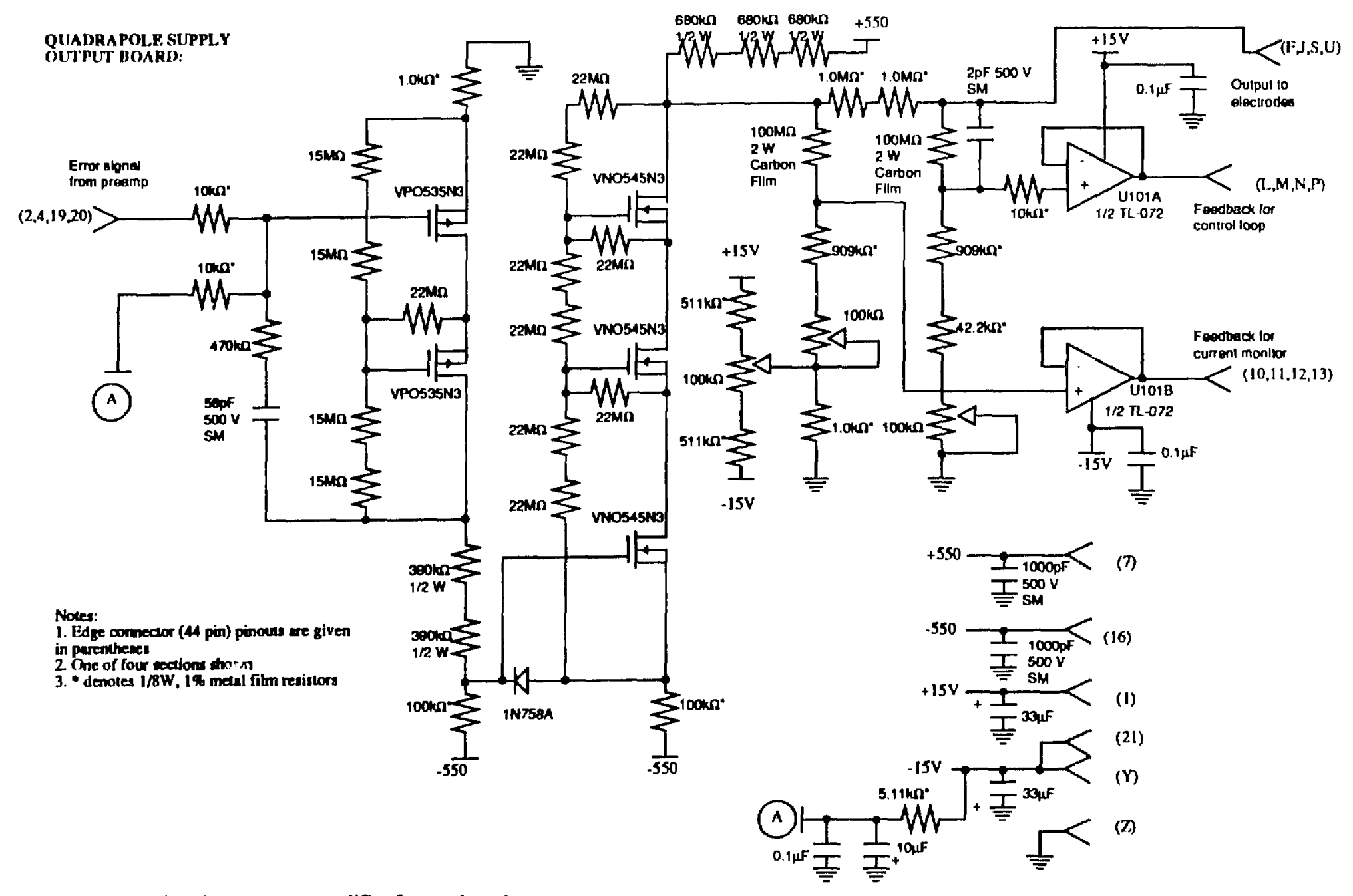

Figure C.9c High voltage output amplifier for quadrupole power supply. 
The circuit in the feedback loop of the power supply is essentially a high-gain comparator followed by a two-stage high-voltage amplifier. With high DC gain and multiple stages of amplification in a feedback loop there is a danger of instability. The feedback loop is stabilized by lowering the comparator op-amp's dominant pole to about $.003 \mathrm{~Hz}$ and by lag-lead compensating the first high voltage amplifier stage. Additionally, the 100:1 voltage divider is bypassed with a capacitor to reduce phase shift and increase feedback for higher frequencies. This zero which this introduces (at about $600 \mathrm{~Hz}$ ) approximately cancels the pole introduced by the first stage lag compensation. The first stage lead introduces a zero at about $6 \mathrm{kHz}$. This helps to limit the phase shift introduced by the second stage transistors. (Their Miller capacitances introduce a pole at about $4 \mathrm{kHz}$.) The Miller capacitance of the first stage devices introduces a pole at around $30 \mathrm{kHz}$. The voltage divider lag introduces an additional pole at about $60 \mathrm{kHz}$. When all of these effects are included, the two-stage high voltage amplifier (measured from input to voltage divider output) reaches a $90^{\circ}$ phase lag at about $40 \mathrm{kHz}$ with a gain of about 1 (DC gain is about 10). Including the $90^{\circ}$ phase shift introduced by the comparator, the open loop phase shift would reach $180^{\circ}$ at about $40 \mathrm{kHz}$. However, an additional lead compensation has been introduced in the comparator stage, with a zero at about $600 \mathrm{~Hz}$. The loop reaches a $180^{\circ}$ phase shift at about $300 \mathrm{kHz}$, with a gain of about $-26 \mathrm{~dB}$. Unity gain is reached at about $15 \mathrm{kHz}$, with a phase lag of about $80^{\circ}$. These large gain and phase margins are sufficient to ensure stability in spite of the significant shifting of the Miller-capacitance poles due to changes in FET bias. (The Miller capacitance is a function of the thickness of the FET's depletion zone, which is a function of bias $v^{\prime}$ tage.) The circuit is stable driving capacitive loads of up to $1000 \mathrm{pF}$ at any voltage between +500 and -500 volts. Open-loop DC gain is about $120 \mathrm{~dB}$. The open-loop gain at $60 \mathrm{~Hz}$ is about $50(34 \mathrm{~dB})$; output ripple is on the order of $10 \mathrm{mV}$. The output voltages should change no more than a volt due to temperature changes of $10^{\circ} \mathrm{C}$.

\section{C.5 Low Current Circuitry}

A number of circuits were needed to measure low currents in the cyclotron. A commercial picoammeter (Keithley model 480) with a minimum resolution of $1 \mathrm{pA}$ was used to measure currents on the dee probe, electrostatic mirror, and Wien filter. A dual multi-input picoammeter (Fig. C.10) was built to measure currents at other locations 
(primarily probes on each side of the injector's entrance and on one side of the inner deflector and extractor entrance). This uses a low current, low bias IC op-amp (Harris HA-5180) on a PC board etched with a guard ring and also has a minimum resolution of 1 pA. A similar, battery operated circuit was built and electrically insulated in a small box for measurements of low currents on high voltage electrodes. For higher current measurements (1 $\mathrm{nA}$ or so) at high voltages a DVM was also used, placed inside a protective enclosure and connected in-line as a voltmeter. The current could be calculated easily from the voltage reading and the meter's input impedance. 


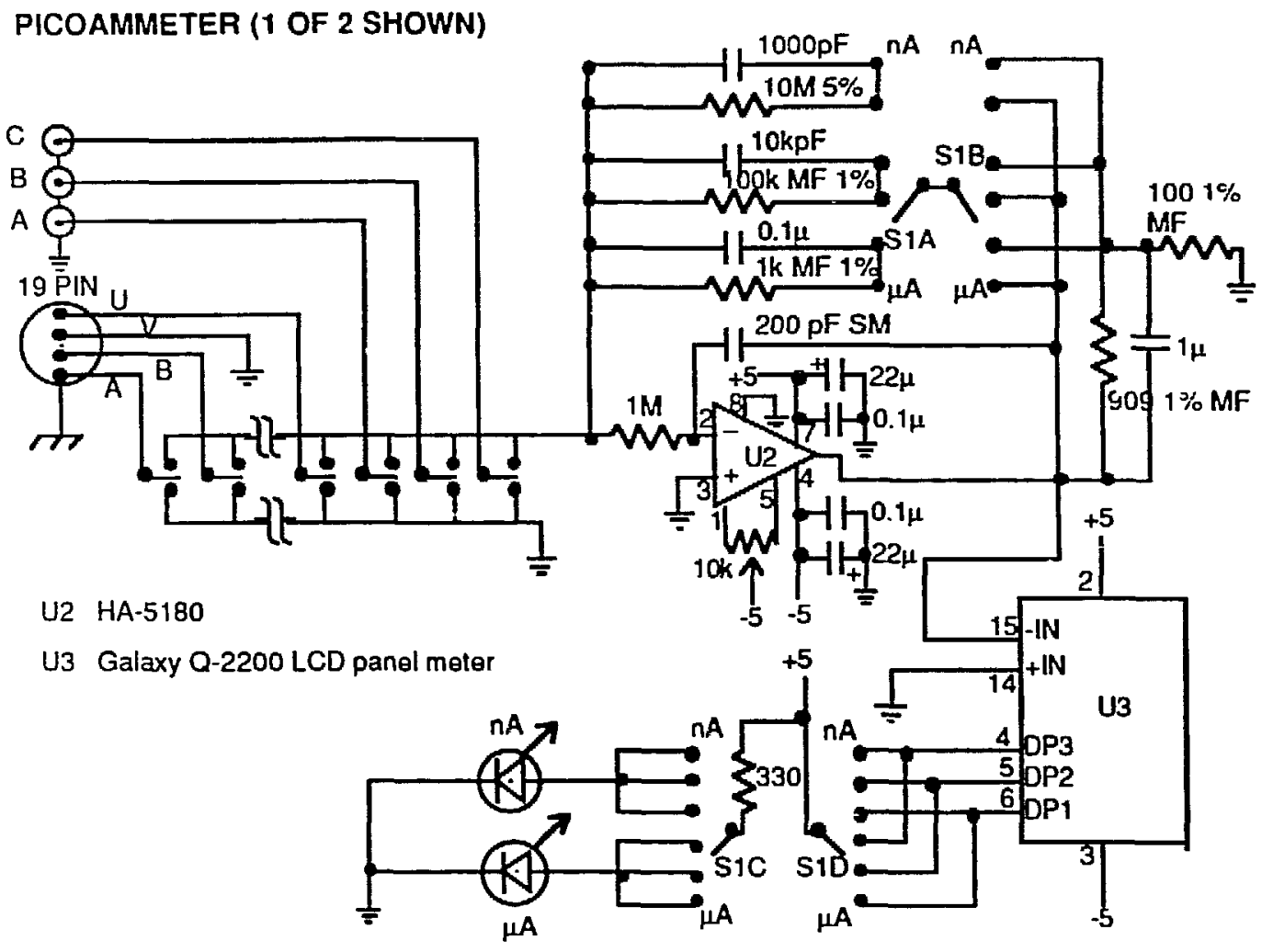

PICOAMMETER POWER SUPPLY

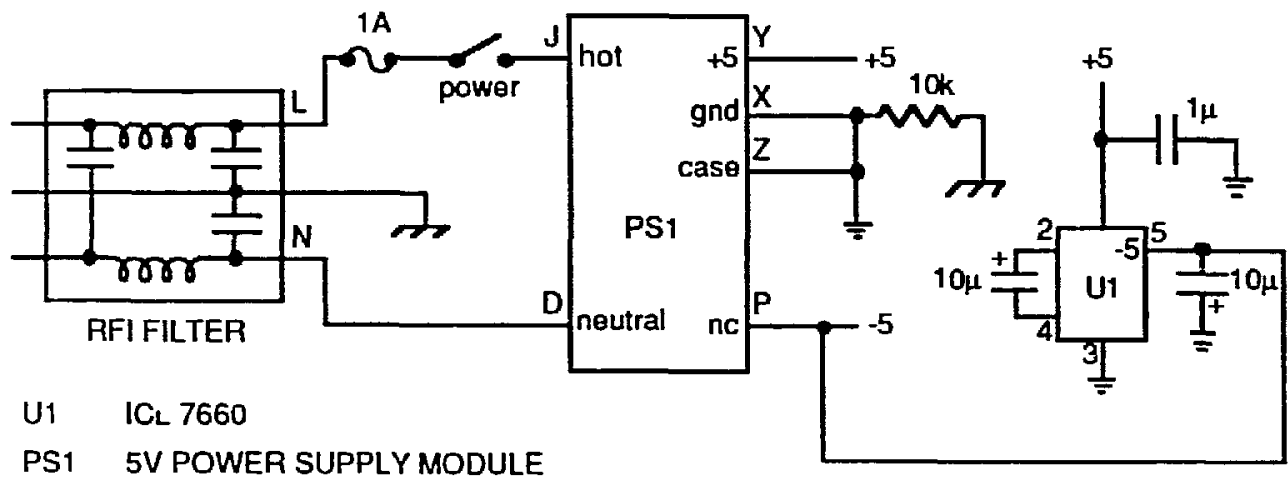

Figure C.10 Circuitry of dual picoammeter. 


\section{C.6 Other Circuitry}

A simple temperature measurement circuit was built (Fig. C.11). The detector was baked out by wrapping heating tape around the box; this circuit enabled monitoring the temperature of the detector assembly.

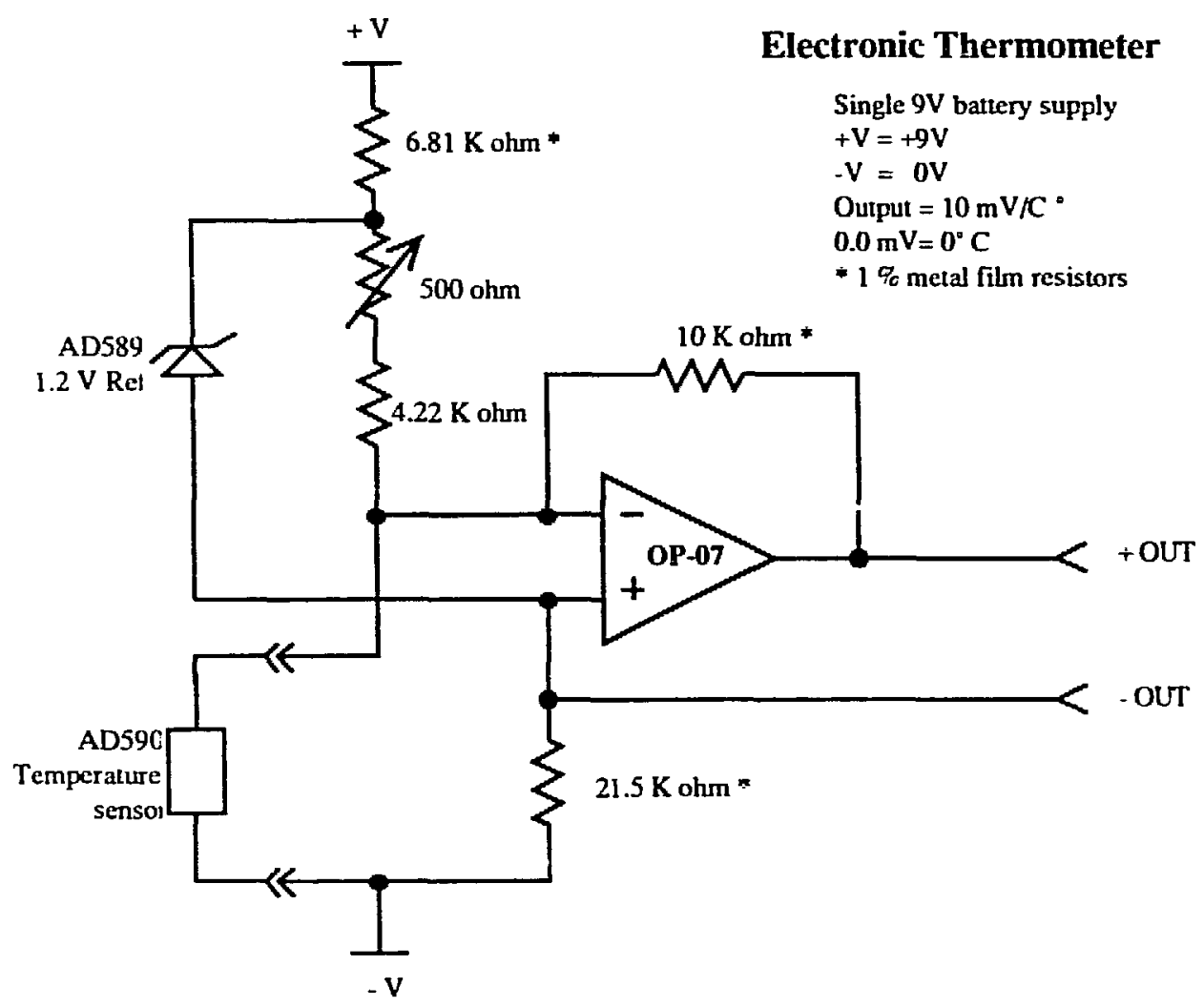

Figure C.11 Circuitry of thermometer circuit used to monitor detector assembly temperature during baking. 


\section{Appendix D: Ion Optical Calculations}

D.1 Ion Optics Overview

Ion optics is very similar to photon optics. In each, the instantaneous state of a paraxial photon or ion may be described by a two-dimensional vector composed of the particle's distance from the optic axis and its angle of motion with respect to the optic axis. The effect of an optical element may be represented by a $2 \times 2$ matrix; the state of a particle after traversing the optical element is found by muitiplying the vector representing the particle's initial state by the matrix. Matrices may be cascaded together to describe the net effect of an entire optical system; the effect of the system on an input ion is found by simply multiplying an input ion's vector this system matrix.

Photon optical systems are generally rotationally symmetric, so this simple description is sufficient. Ion optical systems are often not rotationally symmetric. Thus separate vectors and matrices are needed for each of the two off-axis dimensions. Additionally, it is useful in ion optics to add a third component to the vectors and matrices to represent a perturbation in ion velocity (or momentum or energy), since the focusing of ion optical elements is often highly velocity dependent.

Before the focusing characteristics of an ion optical element can be calculated, the zeroth-order behavior of the element must be understood. Zeroth-order calculations simply solve for the path of an ion traveling along the optic axis of the system. For an einzel lens, quadrupole lens, or Wien filter the zeroth order solution is simply a straight line through the element.

\section{D.2 First Order Calculations}

In this analysis ions it will be assumed that ions travel along the $z$-axis. An ion is denoted by a three-vector composed of $x$ (its offset from the optic axis), $x^{\prime}$ (the angle of its path with respect to the optic axis, equal to $d x / d s$ ) and $d p / p$ (fractional z-momentum offset). We will use $3 \times 3$ matrices to describe optical elements.

The elements of these matrices can be found by first expressing the equations of motion of a general ion in the optical element. The parameters are assumed to deviate only small amounts from the fiducial values (the paraxial approximation) and are written as a 
sum of the fiducial value and a small perturbation. The equation of the fiducial path is then used to eliminate fiducial values from the equations. Terms are retained to first order, and the equations are solved to yield matrix elements.

As an example, we will consider a Wien filter (also known as a crossed field separator or velocity selector). With the ion traveling along the $z$-axis, the electric field applying a force in the $+x$ direction, and the magnetic field applying a force in the $-x$ direction, the equation of motion is:

$$
m \vec{a}=\vec{F}=\vec{F}_{B}+\vec{F}_{E}=\left(q E-q v_{z} B\right) \hat{x}+\left(q v_{x} B\right) \hat{z}
$$

Breaking this into components:

$$
\begin{gathered}
m \frac{d^{2} x}{d t^{2}}=q E-q v_{z} B \\
m \frac{d^{2} z}{d t^{2}}=q v_{x} B \\
m \frac{d^{2} y}{d t^{2}}=0
\end{gathered}
$$

These equations may be rewritten with spatial derivatives rather than time derivatives:

$$
\begin{gathered}
m v_{2}\left(v_{2} \frac{d^{2} x}{d s^{2}}+\frac{d x}{d s} \frac{d v_{z}}{d s}\right)=q E-q v_{z} B \\
m v_{2} \frac{d v_{z}}{d s}=q v_{2} \frac{d x}{d s} B
\end{gathered}
$$

where the $y$ equation has been ignored since there are no forces in the $y$ direction.

Now one can substitute variables which explicitly show the zeroth and first order terms:

$$
\begin{gathered}
v_{z}=v_{z 0}+v_{z 1} \\
x=x_{1}
\end{gathered}
$$

where the numeric subscript denotes the order of the term. Zeroth-order terms are the parameters of the fiducial ion and are assumed constant, first-order terms will be assumed 
non-constant (i.e. $x_{1}$ may be a function of $t$ or $z$; this avoids the need to follow $x_{1}{ }^{\prime}$ as an independent variable). Making these substitutions in (D.3) and separating into zeroth and first order terms:

$$
\begin{gathered}
0=q E-q v_{z 0} B \\
m v_{z 0}\left(v_{z 0} \frac{d^{2} x_{1}}{d s^{2}}\right)=-q v_{z 1} B \\
m \frac{d v_{z 1}}{d s}=q \frac{d x_{1}}{d s} B
\end{gathered}
$$

utilizing the fact that $v_{z 0}$ is constant and neglecting second-order terms. Notice that (D.4a) is just the equation of a fiducial ion. It is obvious from (D.4a) that a Wien filter is a true velocity selector; the only ion-dependent parameter in this equation is the velocity.

Equations (D.4b,c) are the desired first-order equations. These may be rewritten slightly as:

$$
\begin{gathered}
\frac{d^{2} x_{1}}{d s^{2}}=-\frac{\omega_{0} v_{z 1}}{v_{z 0}{ }^{2}} \\
\frac{d v_{z 1}}{d s}=\omega_{0} \frac{d x_{1}}{d s}
\end{gathered}
$$

where $\omega_{0}=\frac{q B}{m}$ has been substituted.

Integration of (D.5b) yields:

$$
v_{z 1}=\omega_{0} x_{1}+C
$$

which may be substituted into (D.5a) to yield:

$$
\frac{d^{2} x_{1}}{d s^{2}}+\frac{\omega_{0}^{2}}{v_{z 0}{ }^{2}} x_{1}=-\frac{\omega_{0} C}{v_{z 0}^{2}}
$$

The solutions to this are:

$$
\begin{aligned}
& x_{1}=A_{1} \cos (k s)+A_{2} \sin (k s)-\frac{C}{\omega_{0}} \\
& v_{z 1}=\omega_{0} A_{1} \cos (k s)+\omega_{0} A_{2} \sin (k s)
\end{aligned}
$$

where: 


$$
k=\frac{\omega_{0}}{v_{z 0}}=\frac{1}{r_{c}}
$$

Equation (D.8a) may be differentiated to yield an equation for $\frac{d x}{d s}$, which we will write as $x^{\prime}$, and $d p / p$ can be calculated knowing that $d p / p=\frac{v_{z 1}}{v_{z 0}}$ to first order. After substituting for $r_{c}=\frac{v_{z 0}}{\omega_{0}}$ and $k=\frac{1}{r_{c}}$, we finally arrive at the first-order equations for ion motion in a Wien filter:

$$
\begin{gathered}
x_{1}=A_{1} \cos (k s)+A_{2} \sin (k s)-\frac{C}{\omega_{0}} \\
x_{1}{ }^{\prime}=\frac{A_{2}}{r_{c}} \cos (k s)-\frac{A_{1}}{r_{i}} \sin (k s) \\
\frac{d p}{p}=\frac{A_{1}}{r_{c}} \cos (k s)+\frac{A_{2}}{r_{c}} \sin (k s)
\end{gathered}
$$

Equations (D.9) for $x_{1}, x_{1}{ }^{\prime}$, and $d p / p$ may now be used to calculate matrix elements. It is convenient to start with rays initially having a deviation in only one of the three dimensions. For example, try a ray with $\left(x_{1}\right)_{0} \neq 0$ and $\left(x_{1}\right)_{0}=(d p / p)_{0}=0$. Then:

$$
\begin{gathered}
x_{1}=\left(x_{1}\right)_{0} \\
x_{1}{ }^{\prime}=0 \\
d p / p=0
\end{gathered}
$$

So $x_{1}$ has a constant value equal to its initial value. In the same way, we can calculate the case where $\left.\left(x_{1}\right)^{\prime}\right) \neq 0$ :

$$
\begin{aligned}
& x_{1}=A_{2} \sin (k s) \\
& x_{1}{ }^{\prime}=\frac{A_{2}}{r_{c}} \cos (k s) \\
& \frac{d p}{p}=\frac{A_{2}}{r_{c}} \sin (k s)
\end{aligned}
$$

where:

$$
\left(x_{1}^{\prime}\right)_{0}=\frac{A_{2}}{r_{c}}
$$


and the case where $(d p / p)_{0} \neq 0$ :

$$
\begin{aligned}
& x_{1}=A_{1}(\cos (k s)-1) \\
& x_{1}^{\prime}=-k A_{1} \sin (k s) \\
& \frac{d p}{p}=\omega_{0} A_{1} \cos (k s)
\end{aligned}
$$

where:

$$
\left(\frac{d p}{p}\right)_{0}=\frac{A_{1}}{r_{c}}
$$

The above results may be combined and expressed in matrix form:

$$
\left[\begin{array}{ccc}
1 & r_{c} \sin (k s) & r_{c}(\cos (k s)-1) \\
0 & \cos (k s) & -\sin (k s) \\
0 & \sin (k s) & \cos (k s)
\end{array}\right]
$$

It appears that we are done and that (D.10) is the matrix we have been seeking. But this is not the case. An additional, subtle effect must be considered. When the ions cross into and out of the Wien filter, they cross electric and magnetic fringe fields which affect their motion. The details of the field profiles will in general create aberrations which are second-order or higher; these may be neglected in the derivation of a first-order ray-trace matrix. However, the integrated effect of crossing these fringe fields does produce a firstorder effect which must be accounted for.

In order to calculate this effect, we assume that the fringe field region is quite short in extent and that ions do not deflect appreciably in it. As an ion crosses this fringe field, it will either gain or lose velocity due to the crossing of electric field lines. This will affect the ion angle, $x_{1}$. Thus, there is a perturbation to beam parameters on entry and exit of the Wien filter.

Assume that a fiducial ion enters the Wien filter along a path of constant electric potential. An ion which enters with an offset, $\left(x_{1}\right)_{0}$, will experience a potential change $U_{1}$, which may be expressed to first order as:

$$
U_{1}=-\left(x_{1}\right)_{0} q E=\left(x_{1}\right)_{0} q v_{20} B
$$


where the equation of the fiducial ion (D.4a) has been used to eliminate $E$. We may also calculate $U_{1}$ from the first order change in kinetic energy:

$$
U=U_{0}+U_{1}=\frac{1}{2} m\left(v_{z 0}+v_{z 1}\right)^{2}=\frac{1}{2} m v_{z 0}^{2}+m v_{z 0} v_{z 1}+\cdots
$$

Thus, from (D.12), $U_{1}=m v_{z 0} v_{z 1}$. Substituting this in (D.11), we find:

$$
v_{21}=x_{1} \frac{q B}{m}=\omega_{0} x_{1}
$$

or:

$$
\frac{d p}{p}=\frac{x}{r_{c}}
$$

This $d p / p$ may affect $x_{1}{ }^{\prime}$. Realizing that the transverse velocity, $\frac{d x}{d t}$, must be constant as an ion crosses into or out of the filter, we may write $x_{1}{ }^{\prime}$ as:

$$
x_{1}{ }^{\prime}=\frac{d x}{d s}=\frac{d x}{d t} \frac{1}{v_{z}}=\frac{d x}{d t} \frac{1}{v_{z 0}+v_{z 1}}=\frac{d x}{d t} \frac{1}{v_{z 0}}\left(1-\frac{v_{z 1}}{v_{z 0}}\right)
$$

Breaking this into zeroth and first-order terms:

$$
\begin{gathered}
x_{0}{ }^{\prime}=\frac{d x}{d t} \frac{1}{v_{z 0}} \\
x_{1}{ }^{\prime}=-x_{0} \frac{v_{z 1}}{v_{z 0}}
\end{gathered}
$$

But, since $x_{0}{ }^{\prime}$ is zero (the fiducial ions go through the Wien filter with no deflection), $x_{1}{ }^{\prime}$ is also zero and there is no change in $x^{\prime}$ to first order. The only parameter which is affected by crossing field lines into or out of the Wien filter is $d p / p$, and the perturbation to $d p / p$ depends only on $x_{1}$.

These equations may be expressed as a matrix which describes the effect of crossing the fringe fields on entry into the Wien filter:

$$
\left[\begin{array}{ccc}
1 & 0 & 0 \\
0 & 1 & 0 \\
\frac{1}{r_{c}} & 0 & 1
\end{array}\right]
$$


A matrix describing the effects of exiting the filter can be derived in the same way: the result is the same except for a sign change in the lower left matrix element.

The true net effect of the Wien filter is found by cascading matrices of the form (D.16) with (D.10) to represent entrance, field region, and exit:

$$
\left[\begin{array}{ccc}
1 & \mathrm{C} & 0 \\
0 & 1 & 0 \\
-\frac{1}{r_{c}} & 0 & 1
\end{array}\right]\left[\begin{array}{ccc}
1 & r_{c} \sin (k s) & r_{c}(\cos (k s)-1) \\
0 & \cos (k s) & -\sin (k s) \\
0 & \sin (k s) & \cos (k s)
\end{array}\right]\left[\begin{array}{ccc}
1 & 0 & 0 \\
0 & 1 & 0 \\
\frac{1}{r_{c}} & 0 & 1
\end{array}\right]
$$

When this is done, the final matrix for a Wien filter is obtained:

$$
\left[\begin{array}{ccc}
\cos \frac{L}{r_{c}} & r_{c} \sin \frac{L}{r_{c}} & r_{c}\left(\cos \frac{L}{r_{c}}-1\right) \\
-\frac{1}{r_{c}} \sin \frac{L}{r_{c}} & \cos \frac{L}{r_{c}} & -\sin \frac{L}{r_{c}} \\
0 & 0 & 1
\end{array}\right]
$$

where $L$ is the Wien filter length. Notice that the bottom row is $(001)$ as it should be; for any optical element with static fields and with the beam entering and exiting in regions of the same potential there should be no change in beam energy or in $d p / p$.

This same procedure may be followed to derive the focusing matrices for other optical elements of interest, some of which are included in Appendix E.

\section{D.3 Large Velocity Deviations}

The above first order analysis applies to small deviations from the fiducial parameters. At times, however, it may be necessary to deal with larger perturbations, especially in velocity. For this the equations of motion for the ion (D.2) must be reexamined. Assuming an ion entering on-axis with no angular deviation, but with an arbitrary velocity perturbation, the temporal solutions to these equations are easily derived:

$$
z=\frac{A}{\omega} \sin (\omega t)+\frac{E}{B} t
$$




$$
\begin{gathered}
x=\frac{A}{\omega}(\cos (\omega t)-1) \\
v_{x}=-A \sin (\omega x)
\end{gathered}
$$

where:

$$
\begin{aligned}
& A=v_{i}-\frac{E}{B} \\
& v_{i}=\text { initial ion velocity (in z-direction) } \\
& \omega=\frac{q B}{m} \text { (for ion of interest) }
\end{aligned}
$$

However, the crossing of electric field lines as an ion exits the filter must again be dealt with, as mentioned above. (We have assumed entry on-axis, so there is no effect on entering the filte-) The effect may be modeled as causing an abrupt change in $z$-velocity, while leaving the $x$-velocity unchanged. This will instantaneously change the direction of an ion exiting the Wien filter. The Wien filter will not change the energy of an ion. Thus, the exit velocity is given by:

$$
v_{z}^{2}+v_{x}^{2}=v_{i}^{2}
$$

The exit direction (after fringe fields) may be found by combining (D.18c) and (D.19):

$$
\theta_{f}=\tan ^{-1} \frac{v_{x}}{v_{z}}=\tan ^{-1}\left(\frac{-A \sin (\omega t)}{\sqrt{v_{i}^{2}-A^{2} \sin ^{2}(\omega t)}}\right)
$$

Collecting (D.18) and (D.20) together:

$$
\begin{gathered}
L=\frac{A}{\omega} \sin (\omega t)+\frac{E}{B} t \\
x_{f}=\frac{A}{\omega}(\cos (\omega t)-1) \\
\theta_{f}=\tan ^{-1}\left(\frac{-A \sin (\omega t)}{\sqrt{v_{i}^{2}-A^{2} \sin ^{2}(\omega t)}}\right)
\end{gathered}
$$

where:

$L=$ length of Wien filter, 


$$
\begin{aligned}
& A=v_{i}-\frac{E}{B} \\
& \omega=\frac{q B}{m} \text { (for ion of interest) } \\
& v_{i}=\text { initial ion velocity (in z-direction) } \\
& x_{f}=\text { final x-offset } \\
& \theta_{f}=\text { final angle }
\end{aligned}
$$

This (D.21) represents three equations in three unknowns. To solve them for an arbitrary ion, the nonlinear equation (D.2la) must first be solved for $t$; this may be substituted in (D.2lb,c) to find the ion's offset and angle upon exiting the Wien filter.

For small velocity shifts $d v,(\mathrm{D} .21 \mathrm{a}-\mathrm{C})$ reduce to:

$$
\begin{gathered}
L=\frac{E}{B} t=v_{z} t \\
x_{f}=\frac{d v}{\omega}(\cos (\omega t)-1)=\frac{d v}{\omega}\left(\cos \frac{\omega L}{v_{z}}-1\right) \\
\theta_{f}=\frac{d v \sin \frac{\omega L}{v_{z}}}{v_{z}}
\end{gathered}
$$

which are in a different form, but agree with (D.17).

\section{D.4 A Practical Wien Filter}

To use a Wien filter as a velocity separation device there must be a slit or aperture to allow the desired ions to pass, while off-velocity ions miss the slit. This slit should be placed at the optical focus of the selected ions for best separation. Generally a slit is placed before the Wien filter as well; this object slit is imaged onto the output (or image) slit.

Thus, in general, a Wien filter system will have a slit, a drift length, the Wien filter, another drift length, and the second slit. Restricting ourselves to the case where the two drift lengths are equal, the first-order optical matrix of this system may be calculated by cascading the matrices for the Wien filter and drift lengths: 


$$
\begin{aligned}
& {\left[\begin{array}{ccc}
1 & d & 0 \\
0 & 1 & 0 \\
0 & 0 & 1
\end{array}\right]\left[\begin{array}{ccc}
\cos \frac{L}{r_{c}} & r_{c} \sin \frac{L}{r_{c}} & r_{c}\left(\cos \frac{L}{r_{c}}-1\right) \\
-\frac{1}{r_{c}} \sin \frac{L}{r_{c}} & \cos \frac{L}{r_{c}} & -\sin \frac{L}{r_{c}} \\
0 & 0 & 1
\end{array}\right]\left[\begin{array}{ccc}
1 & d & 0 \\
0 & 1 & 0 \\
0 & 0 & 1
\end{array}\right]=} \\
& {\left[\begin{array}{ccc}
\cos \frac{L}{r_{c}}-\frac{d}{r_{c}} \sin \frac{L}{r_{c}} & \left(r_{c}-\frac{d^{2}}{r_{c}}\right) \sin \frac{L}{r_{c}}+2 d \cos \frac{L}{r_{c}} & r_{c}\left(\cos \frac{L}{r_{c}}-1\right)-d \sin \frac{L}{r_{c}} \\
-\frac{1}{r_{c}} \sin \frac{L}{r_{c}} & \cos \frac{L}{r_{c}}-\frac{d}{r_{c}} \sin \frac{L}{r_{c}} & -\sin \frac{L}{r_{c}} \\
0 & 0 & 1
\end{array}\right]}
\end{aligned}
$$

where:

$$
\begin{aligned}
& L=\text { Wien filter length } \\
& r_{c}=\text { cyclotron radius of selected ions }=v_{i} / \omega_{c} \\
& v_{i}=\text { incident ion velocity of selected ions } \\
& d=\text { drift length }
\end{aligned}
$$

In order for this system to focus the input slit onto the output slit, we require that the final position be independent of the initial angle, i.e. in (D.23) the matrix element $M_{12}=0$. Thus:

$$
\left(r_{c}-\frac{d^{2}}{r_{c}}\right) \sin \frac{L}{r_{c}}+2 d \cos \frac{L}{r_{c}}=0
$$

This, of course, can be satisfied in many ways. Two choices of parameters immediately suggest themselves, however. Firstly, one could choose $d=0$, i.e. no drift lengths. In this case the slits are physically located at the beginning and end of the crossed fields. Then (D.24) reduces to:

$$
r_{c} \sin \frac{L}{r_{c}}=0, \text { or } L=\pi r_{c}
$$

For small velocity shifts $d v$, the ion separation at the second slit is given by (D.22):

$$
x=\frac{d v}{\omega}(\cos \pi-1)=-2 \frac{d v}{\omega}
$$


Alternatively, one could choose the case $d=r_{C}$. Then the focusing condition is:

$$
2 r_{c} \cos \frac{L}{r_{c}}=0, \text { or } L=\frac{\pi}{2} r_{c}
$$

And for small velocity shifts, from (D.22) we find at the end of the crossed-field region:

$$
\begin{gathered}
x=\frac{d v}{\omega}\left(\cos \frac{\pi}{2}-1\right)=-\frac{d v}{\omega} \\
\theta=-\frac{d v}{v_{2}} \sin \frac{\pi}{2}=-\frac{d v}{v_{z}}
\end{gathered}
$$

After the final drift length of $d=r_{l}$ to the image plane, the separation is:

$$
x_{\mathrm{tor}}=-\frac{d v}{\omega}-\frac{d v r_{c}}{v_{z}}=-2 \frac{d v}{\omega}
$$

Thus the separation of off-velocity ions is the same for both cases. The second case has a shorter field region by a factor of two but a longer overall length by a factor of 1.14 .

This result may be expressed in terms of a mass difference as well, assuming all ions have equal energy. (All ions do have approximately the same energy. Since we use a cesium sputter ion source at a $-5 \mathrm{kV}$ bias, all ions must have at least $5 \mathrm{keV}$ energy. There will be very few multiply-charged negative ions. The singly-charged ions may have a few $\mathrm{eV}$ extra kinetic energy due to the $\mathrm{Cs}$ ion collisions which generate them, but this should not exceed 10 or $20 \mathrm{eV}$.) Using the relations $r_{c}=v_{i} / \omega_{c}$ and $T=1 / 2 \mathrm{mi}^{2}$, w: find:

$$
x_{\mathrm{tot}}=-2 r_{c} \frac{d v}{v_{2}}=r_{c} \frac{d m}{m_{0}}
$$

\section{D.5 Adjustment of W'ien Filter Focus}

Once such a filter is installed, the desired beam must be imaged at the output slit. In general, it will not be imaged exactly at the slit due to measurement and assembly errors. There are two ways in which this may be adjusted without removing the filter and making mechanical adjustments. Both methods rely on changing the beam energy (velocity). 
Firstly, the incident beam energy can be changed, if possible. This will change $v_{2}$ and $r_{i}$ inside the filter. This is the most direct way of adjusting the image position, although it may not be possible due to other system constraints, external to the Wien filter. The sensitivity of image position to beam energy may be calculated by cascading the system matrix given above with a subsequent drift of length $z$, and calculating the derivative $\frac{d z}{d T}$. If this is done, the $M_{12}$ matrix element of (D.23) (which must be zero for a focused image) becomes:

$$
\left(r_{c}-\frac{d(d+z)}{r_{c}}\right) \sin \frac{L}{r_{c}}+(2 d+z) \cos \frac{L}{r_{c}}=0
$$

The derivative $\frac{d z}{d T}$ may be found by taking differentials, fixing every parameter except $T$ (hence $v$ ) and $z$. One then obtains:

$$
\frac{d z}{r_{c}}=\frac{1}{2} \frac{d T}{T} \frac{\left(1+\frac{d^{2}}{r_{c}{ }^{2}}+\frac{2 d L}{r_{c}{ }^{2}}\right) \sin \frac{L}{r_{c}}-\frac{L}{r_{c}}\left(1-\frac{d^{2}}{r_{c}{ }^{2}}\right) \cos \frac{L}{r_{c}}}{\frac{d}{r_{c}} \sin \frac{L}{r_{c}}-\cos \frac{L}{r_{c}}}
$$

For the first case, where $t=0$ and $L=\pi r_{c}$, this reduces to:

$$
\frac{d z}{r_{c}}=\frac{\pi}{2} \frac{d T}{T}
$$

For the case where $d=r_{c}$ and $L=\frac{\pi}{2} r_{c}$, it reduces to:

$$
\frac{d z}{r_{c}}=\frac{(\pi+2)}{2} \frac{d T}{T}
$$

If the incident beam energy cannot be adjusted due to external system constraints, the image position may still be adjusted by changing the ion energy inside the Wien filter. This can be done by offsetting the voltages of the electric field plates in the Wien filter. Calculation of this case is similar to the previous, except that the matrix is more complex. A matrix representing change in beam energy on entrance to the Wien filter must be included in the calculation of the system matrix. This matrix is of the form:

$$
\left[\begin{array}{lll}
1 & 0 & 0 \\
0 & n & 0 \\
0 & 0 & 1
\end{array}\right]
$$


On exiting the Wien filter, a similar matrix must be inserted in which $n$ is replaced by $1 / n$. In these matrices, $n$ is the "refractive index", equal to $\frac{v_{\text {outside }}}{v_{\text {inside }}}$.

When this is done, a new system optical matrix is obtained. The element $M_{12}$ becomes:

$$
\left(n r_{c}-\frac{d(d+z)}{n r_{c}}\right) \sin \frac{L}{r_{c}}+(2 d+z) \cos \frac{L}{r_{c}}=0
$$

The derivative $\frac{d z}{d T}$ may again be found by taking differentials, fixing every parameter except $T_{\text {in }}$ (hence $v_{\text {in }}, r$, and $n$ ) and $z$. One then obtains:

$$
\frac{d z}{L}=\frac{1}{2} \frac{d T_{\mathrm{in}}}{T_{\mathrm{in}}} \frac{\frac{2 d}{r_{c}} \sin \frac{L}{r_{c}}-\left(n-\frac{d^{2}}{n r_{c}^{2}}\right) \cos \frac{L}{r_{c}}}{\frac{d}{n r_{c}} \sin \frac{L}{r_{c}}-\cos \frac{L}{r_{c}}}
$$

For the case $d=0$ and $L=\pi r_{c}$ :

$$
\frac{d z}{L}=\frac{n}{2} \frac{d T_{\text {in }}}{T_{\text {in }}}, \text { or } \frac{d z}{r_{c}}=\frac{n \pi}{2} \frac{d T_{\text {in }}}{T_{\text {in }}}
$$

And for $d=r_{c}$ and $L=\frac{\pi}{2} r_{c}$, it reduces to:

$$
\frac{d z}{L}=n \frac{d T_{\text {in }}}{T_{\text {in }}}, \text { or } \frac{d z}{r_{c}}=\frac{n \pi}{2} \frac{d T_{\text {in }}}{T_{\text {in }}}
$$

where $T_{\mathrm{in}}$ is the beam energy inside the Wien filter. Note that $n$ will generally be unity or near unity. Thus we see that changing the energy inside the Wien filter has roughly the same effectiveness in adjusting image location as does changing the original beam energy.

These derivations regarding focusing apply only for small changes in beam energy and small focus adjustments. For large changes, the approximations which were used do not hold and more detailed calculations are necessary. It should also be realized that adjusting the beam energy in either of these ways will also affect the separation of offvelocity ions. 


\section{Appendix E: Catalog of Matrices for Ion Optical Elements}

In the matrices to follow, $r_{c}=\frac{v_{i}}{\omega_{0}}$ is the cyclotron radius of a fiducial ion, and the components of the three-vectors which are transformed by these matrices are $x, x^{\prime}=d x / d s$, and $d p / p$.

FREE SPACE:

$$
\left[\begin{array}{lll}
1 & d & 0 \\
0 & 1 & 0 \\
0 & 0 & 1
\end{array}\right]
$$

where $d$ is the distance travelled.

MAGNETIC FIELD:

$$
\left[\begin{array}{ccc}
\cos \alpha & r_{c} \sin \alpha & r_{c}(1-\cos \alpha) \\
-\frac{1}{r_{c}} \sin \alpha & \cos \alpha & \sin \alpha \\
0 & 0 & 1
\end{array}\right]
$$

where $\alpha$ is the angle travelled in the magnetic field. This may be expressed in terms of distance travelled by $s=\alpha r_{c}$.

WIEN FILTER:

$$
\left[\begin{array}{ccc}
\cos \frac{L}{r_{c}} & r_{c} \sin \frac{L}{r_{c}} & r_{c}\left(\cos \frac{L}{r_{c}}-1\right) \\
-\frac{1}{r_{c}} \sin \frac{L}{r_{c}} & \cos \frac{L}{r_{c}} & -\sin \frac{L}{r_{c}} \\
0 & 0 & 1
\end{array}\right]
$$

where $\mathrm{L}$ is the Wien filter length. 
CURVED ELECTRIC DEFLECTION CHANNEL IN MAGNETIC FIELD:

$$
\left[\begin{array}{ccc}
\cos B \beta & \frac{|R|}{B} \sin B \beta & r_{c} A(1-\cos B \beta) \\
-\frac{B}{|R|} \sin B \beta & \cos B \beta & \frac{r_{c} A B}{|R|} \sin B \beta \\
0 & 0 & 1
\end{array}\right]
$$

where $R$ is the radius of curvature of the deflection channel, positive if in the same direction as $r_{C}$ and negative if in the other direction; $\beta$ is the angle traversed in the channel; and:

$$
\begin{aligned}
& A=\frac{1-C^{2}}{1+C^{2}} \\
& B=\sqrt{C^{2}+1} \\
& C=\frac{R-r_{c}}{r_{c}}
\end{aligned}
$$

Notice that the Wien filter and the magnetic field matrices are special cases of this matrix.

QUADRUPOLE LENS:

A different matrix is needed for the focusing and defocusing dimension:

$$
\begin{aligned}
& {\left[\begin{array}{ccc}
\cos k L & \frac{1}{k} \sin k L & 0 \\
-k \sin k L & \cos k L & 0 \\
0 & 0 & 1
\end{array}\right]} \\
& {\left[\begin{array}{ccc}
\cosh k L & \frac{1}{k} \sinh k L & 0 \\
-k \sinh k L & \cosh k L & 0 \\
0 & 0 & 1
\end{array}\right]}
\end{aligned}
$$

where: 
$k^{2} \cong \frac{\Phi}{2 T a^{2}}$

$L=$ electrode length

$\Phi=$ total potential difference on lens electrodes

$T=$ beam energy

$a=$ lens aperture

$180^{\circ}$ ELECTROSTATIC MIRROR IN MAGNETIC FIELD:

$$
\left[\begin{array}{ccc}
1 & 3.6 x_{M P} & -7.2 \frac{x_{M P}}{r_{c}} \\
0 & 1 & 0 \\
0 & 0 & 1
\end{array}\right]
$$

where $x_{M P}$ is the distance from the outside of the mirror to the effective "mirror plane", which the ions appear to reflect from. This distance is short of the distance one would expect the ions to travel based on energy considerations by a factor of 1.39 . For example, a $5 \mathrm{keV}$ ion would be expected to go into the uniform electric field of the mirror until it reaches the $5 \mathrm{kV}$ equipotential surface; it appears optically, however, to reflect off of the $3.59 \mathrm{kV}$ equipotential. 


\title{
Appendix F: Beam Bunching
}

\section{A Simple Buncher for the Berkeley Small Cyclotron}

\author{
Chandu A. Karadi \\ Senior Thesis \\ Physics Department, \\ University of California, Berkeley \\ Spring 89
}

\begin{abstract}
A simple buncher and its associated radio frequency electronics has been designed for the Berkeley Small Cyclotron. The buncher should boost the efficiency of the existing system by a factor of four to five, enabling the dating of archaeological samples in a practical amount of time. The buncher will operate over the frequency range of 5 to $15 \mathrm{MHz}$ with mass 14 and mass 12 negative ions at $5 \mathrm{KeV}$. Also included is a discussion on a variation of the simple buncher, dubbed the gap buncher. The gap buncher employs three grids and is driven by a square wave which in the ideal case creates perfect linear bunching of $50 \%$ of the beam down to $0^{\circ}$.
\end{abstract}




\section{F.1. Introduction:}

Today there is much interest in the development of accelerator mass spectroscopy (AMS) which today takes place at large accelerator facilities at a relatively high cost. In 1981 the small cyclotron project began at UC. Berkeley and is now coming close to the point of dating archaeological samples by separating ${ }^{14} \mathrm{C}$ ions from the other background ions $[1,2]$. There is also great current interest in using this device in medical research where the cyclotron could be used to trace a variety of biochemical pathways which have been tagged by ${ }^{14} \mathrm{C}$ [3]. This could help uncover the mechanism behind many metabolic disorders such as schizophrenia and other mental diseases.

At present the system consists of an external negative ion source, einzel lens, Wien filter, electrostatic focusing optics, and $15 \mathrm{~cm}$. radius cyclotron (see Fig. F.1). It was proposed [2] that a buncher should be installed along the beam path to boost the efficiency of the system. At present, we estimate a buncher will increase the efficiency by a factor of four or five. This will allow the dating of archaeological samples in a practical amount of time. Assuming a count rate of about 4 per minute, we estimate that it will take 4.5 hours to date modern carbc $n$ with an accuracy of $3 \%$ [4]. A buncher should speed the process up to one hour.

There are a variety of schemes for bunching ions, ranging from a buncher with only two electrodes [5] to more novel schemes such as a double drift buncher with six electrodes [6]. We decided for a variety of reasons to design a simple buncher with three electrodes with the center electrode driven by a sinusoidal voltage. This design allows the maximum ease in construction and requires the least amount of additional radio-frequency (RF) electronics. Other systems such as harmonic bunchers or double drift bunchers require special RF electronics which are not readily available. The main advantage to these other schemes is that they are able to bunch a large fraction $(>70 \%)$ of the beam down to a phase angle of $+1-6^{\circ}$ to $9^{\circ}[5,6]$. Our cyclotron, though, has a phase acceptance of $\pm 20^{\circ}$ [4] for which a simple buncher will suffice. Ideally (not including losses due to grids) a simple buncher should bunch about $55 \%$ of the beam down into this phase window.

In this paper we will discuss the basic physics of two types of bunchers, the simple buncher and the gap buncher. Then we will go on to a detailed discussion on the design of a complete bunching system employing a simple buncher. 


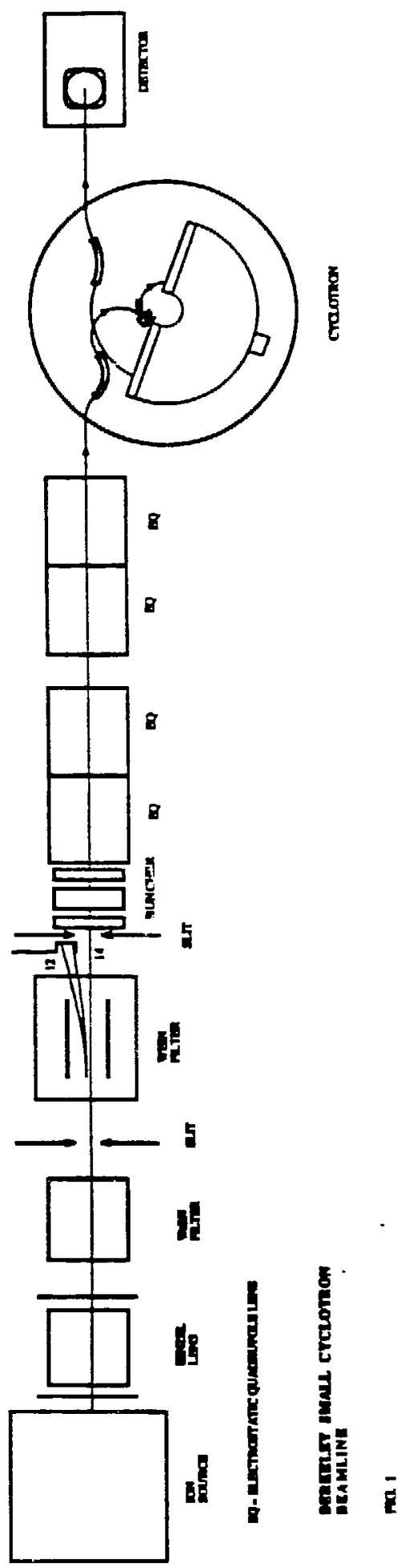

Figure F.1 Berkeley small cyclotron beamline. (See also Fig. 3.1, in body of thesis.) 


\section{F.2. Simple and Gap Bunchers.}

\section{Principles of Operation:}

Here we discuss the basic principles behind the operation of a simple buncher and a gap buncher.

\section{F.2.1 Simple Buncher}

Here is a schematic drawing of a simple buncher.
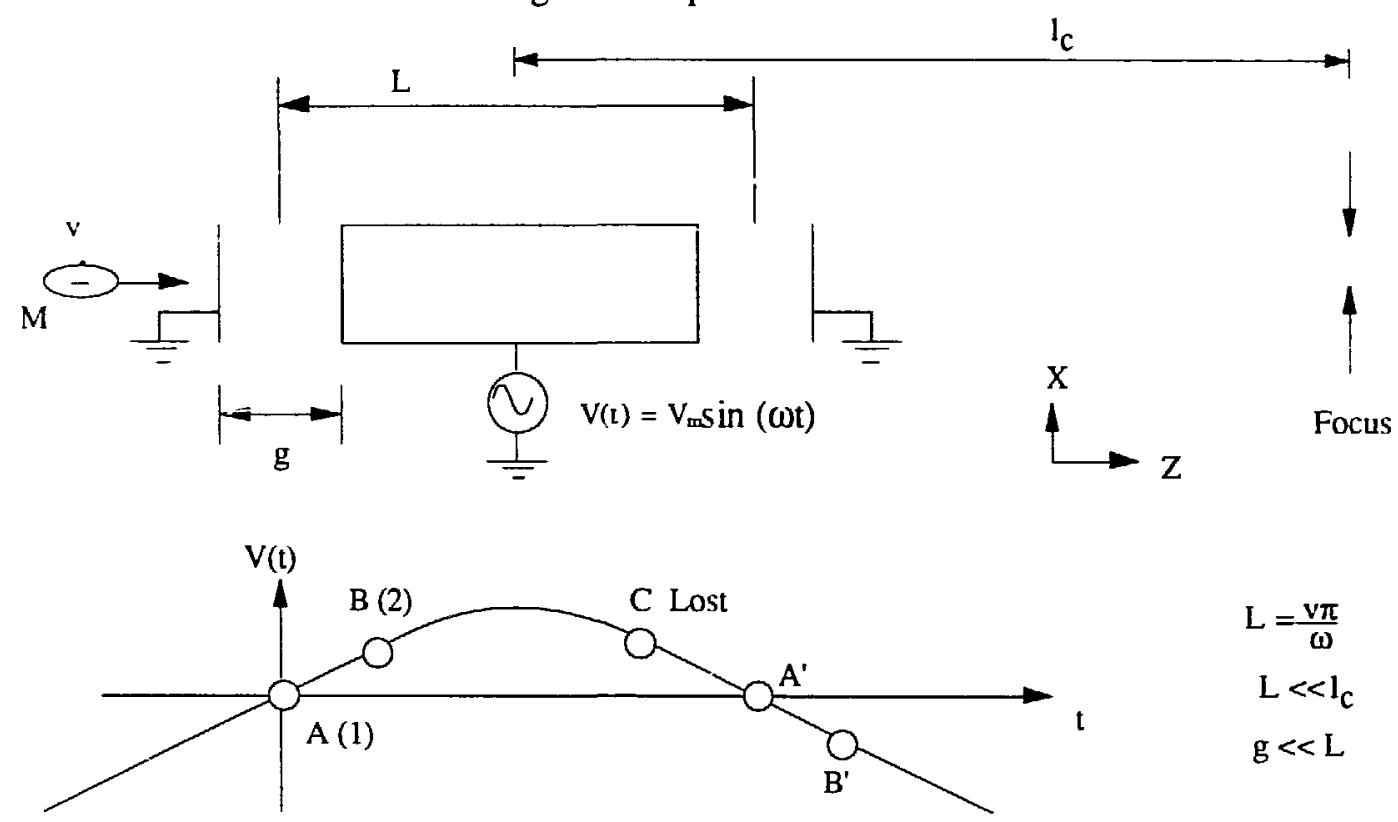

The two outer electrodes are grounded while the center electrode is driven by a sinusoidaly varying voltage $=\mathrm{V}(\mathrm{t})$. Here we assume that the electric fields are confined to the gap region between the tube and the two end plates and that the gap is much smaller than the length of the buncher $(\mathrm{g}<\mathrm{L}$ ). Each gap can be thought of as an infinite parallel plate capacitor. Of course in reality the plates are replaced by grids so that an ion may pass through them (see F.3.1.2 for details). The length of the buncher is such that a singly charged ion (we take a negative ion for concreteness) of mass $=M$ and velocity $=v$, will transverse the length of the buncher $=\mathrm{L}$ in half a cycle of $\mathrm{V}(\mathrm{t})$. Now consider an ion (1) that enters the buncher at point $A$ on the waveform. (1) enters at $A$ and exits at $A^{\prime}$, each time seeing no force, and thus no net bunching. . Now consider the ion (2) which enters at a later time B and exits at B'. (2) sees a positive (z-direction) accelerating force both at the first and second gap. This gives (2) a boost in velocity, allowing him to catch up to (1) once they travel a distance $1_{c}$. This is the basis of the bunching mechanism. Similar considerations show that an ion that that enters before (1) will get a net deaccelerating 
force, allowing the other ions to catch up. We call (1) the center ion, to which the other ions are focused. A simple way to picture the bunching effect is to use phase space diagrams, where we go into the frame of the center ion (1)

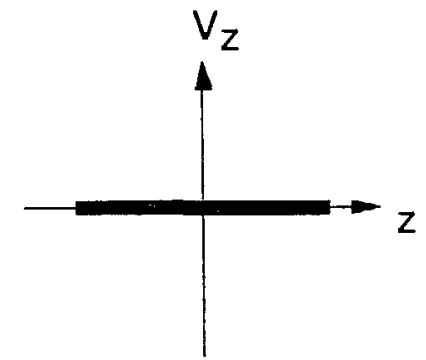

Before Buncher

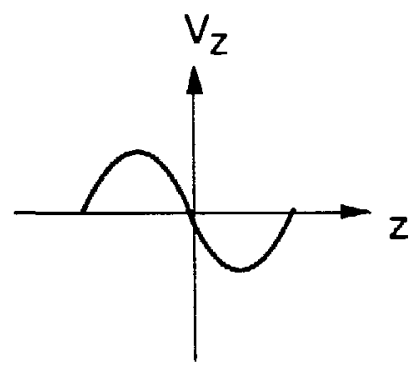

After Buncher

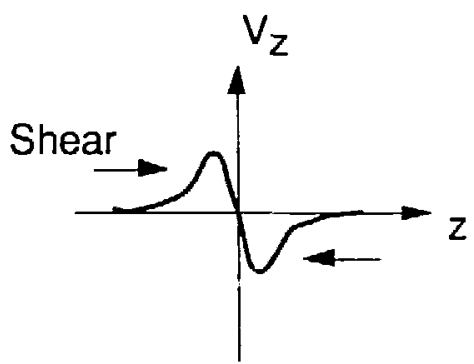

Along Beam Path

Throughout this paper we assume that the beam has no initial velocity spread. After the ions have received a velocity modulation, the sinusoidal shape shears in the $\mathrm{z}$ direction. From this it is obvious that only ions on the linear portion will be perfectly bunched. Notice also that bunching quality degrades rapidly for ions out of phase by more than $90^{\circ}$. If one assumes that distance to the cyclotron $\left(l_{c}\right)$ is much greater than the length of the buncher ( $L$ ), then all the ions within $\pm 100^{\circ}$ of the center ion can be focused down into our phase window of $\pm 20^{\circ}$. Theses numbers were computed by allowing the $90^{\circ}$ ion to reach $0^{c}$, and then computing the fraction of ions which fell into our phase window.

\section{F.2.2 Gap Buncher:}

You can see easily fr'sm above that if one applies a triangle waveform to a simple buncher, one can have linear bunching for $50 \%$ of the beam down to $0^{\circ}$. In the past much effort has gone into adding up harmonics of a particular frequency to create a nearly triangle waveform. Now we turn briefly to a novel idea for a buncher which allows for linear bunching for $50 \%$ of the beam, but does not employ a triangle waveform. This new type of buncher, dubbed gap buncher, could possibly be used in future generations of the small cyclotron. 

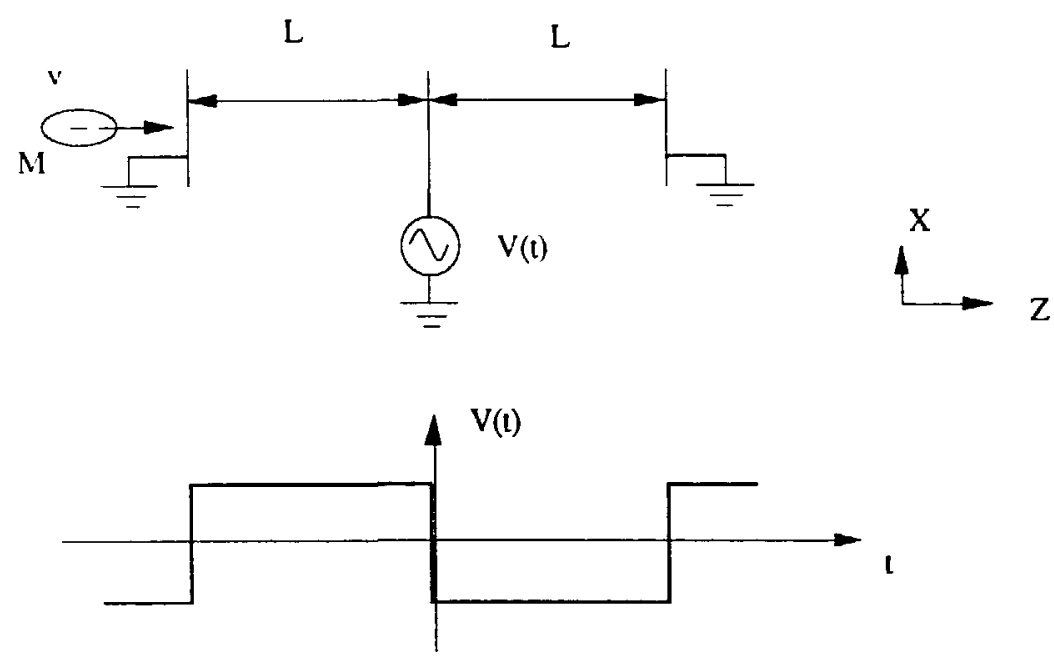

$$
\begin{aligned}
& \mathrm{L}=\frac{\mathrm{v} \tau_{0}}{2} \\
& \tau_{0}=\text { Period of square wave }
\end{aligned}
$$

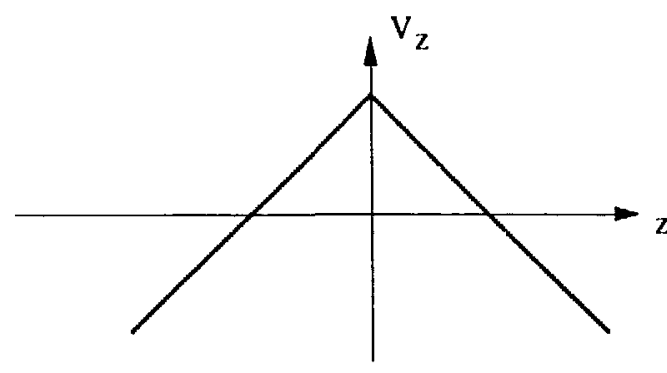

After Buncher

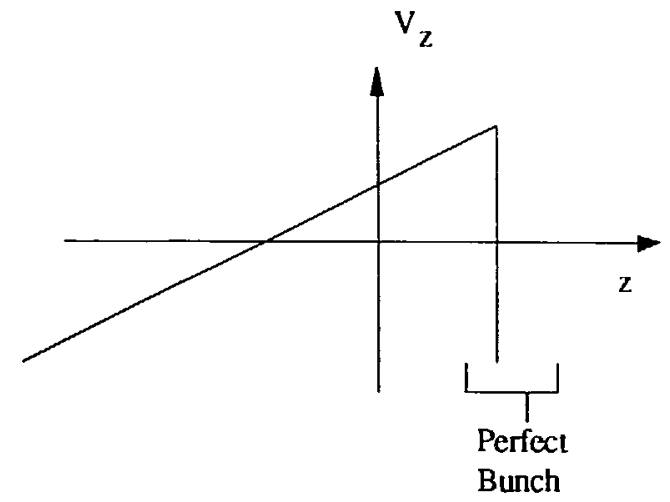

In the frame of ion whichpasses through the center of the buncher at $\mathrm{t}=0$.

Along Beam Path

The gap buncher is driven by a square wave voltage and its center electrode is now a thin grid, so that an ion passing through the gap buncher will see an integrated force. Previously, with the simple buncher, the gaps were assumed to be small, so that this effect was neglected. The integration of a square wave is of course a triangle wave, therefore after the gap buncher the velocity spread of the beam will be a triangle wave. Note also that the length of the gap buncher is twice that of a simple buncher operating at the same frequency. Aside from linear bunching, another attribute of a gap buncher is that it requires only three grids as opposed to the four grids needed in the simple buncher. Grids are normally required to create uniform electric fields, but have the disadvaniage of intercepting the beam. The main disadvantage of the gap buncher is that it requires an RF square wave voltage. In large accelerators the voltages required can be quite large (in excess of 500 volts) which may make it impractical to design the necessary electronics. For our cyclotron, though, the voltages needed are around 100 volts which should make it possible to find the necessary electronics. 
We believe the gap buncher is an interesting idea, and will keep it in mind as further changes are made in the small cyclotron. For now, though, we have decided to use a simple buncher in the current version of the small cyclotron.

\section{F.3. Design of A Simple Buncher:}

The design of a buncher consists of two basic parts, physical design and RF electronics design. First we discuss the physical design and then briefly the electronics which can be bought mostly off the shelf. The basic design parameters are as follows:

1. Bunches at least $50 \%$ of the beam down into a phase window of $\pm 20^{\circ}$

2. Operates over the range of frequencies from $5 \mathrm{MHz}$ to $15 \mathrm{MHz}$

3. Works both with mass 14 and mass 12 negative ions

4. $10 \mathrm{eV}<$ Axial gain $<250 \mathrm{eV}$

5. Angular spread caused by grid wires $<10 \mathrm{mrad}$ (half angle)

6. Uses the minimum amount of grid wires

7. Low voltage operation

8. Small size (less than $5 \mathrm{~cm}$. in length)

9. Simple to build and operate

\section{F.3.1 Physical Design:}

\section{F.3.1.1 Frequency and Mass Effects:}

The first question that arises is what length should we make the buncher since we will be operating over a range of frequencies and masses. Since the cyclotron will only operate at odd harmonics of about $1 \mathrm{MHz}$, a simple minded guess would be to choose a length corresponding to 9 or $11 \mathrm{MHz}$ which is at the middle of our operating range. It turns out for this case that $11 \mathrm{MHz}$ is the better choice for reasons that will be discussed later. When considering mass effects, we primarily consider mass 14 ions since mass 12 will only be running through the buncher during tuning of the machine. It turns out that most of the corrections for off mass operation go like the mass $1 / 2$ which is only an $8 \%$ variation for our specific case.

It is easy to show that the length of the buncher is given by the following equations. 


$$
\begin{aligned}
& \begin{array}{l}
\lambda_{0}=\frac{v}{f_{0}}=\sqrt{\frac{2 T_{0}}{M}} \frac{1}{f_{0}}=\text { wavelength } \\
L=\text { Buncher length }=\frac{\lambda_{0}}{2} \\
\mathrm{f}_{\mathrm{O}}=\text { center frequency }
\end{array} \\
& \mathrm{T}_{\mathrm{O}}=\text { initial kinetic energy } \\
& \mathrm{M}=\text { mass of ion } \\
& \mathrm{v}=\text { ion velocity }
\end{aligned}
$$

For operation at $11 \mathrm{MHz}$, with mass 14 ions at $5 \mathrm{KeV}$, the wavelength is about $2.4 \mathrm{~cm}$ so that $\mathrm{L}$ is about $1.2 \mathrm{~cm}$.

Next we would like to know how much voltage will be necessary to bunch the beam if the injection point to the cyclotron is a distance $l_{c}$ away from the buncher. This too is a simple mechanics problem with the following solution.

$$
\begin{aligned}
& \Delta \mathrm{T}=\text { Necessary KE Gain }=2 \mathrm{~T}_{0} \frac{\mathrm{d}}{\mathrm{l}_{\mathrm{c}}} \\
& \mathrm{V}_{\mathrm{m}}=\text { voltage on buncher }=\Delta \mathrm{T} / 2 \mathrm{e} \\
& \mathrm{T}_{\mathrm{o}}=\text { initial kinetic energy } \\
& \mathrm{l}_{\mathrm{C}}=\text { distance from buncher to cyclotron } \\
& \mathrm{d}=\text { distance which must be bunched } \\
& \mathrm{e}=\text { magnitude of the charge on an electron } \\
& \mathrm{d}<<\mathrm{l}_{\mathrm{c}}
\end{aligned}
$$

As an estimate, we take $d$ to be a $1 / 4$ wavelength since we want to bunch the $90^{\circ}$ ions to about $0^{\circ}$. This gives for $5 \mathrm{KeV}$ ions with $\mathrm{l}_{\mathrm{C}} \approx 100 \mathrm{~cm}$, a necessary gain of $60 \mathrm{eV}$. This means that the voltage on the buncher need only be about 30 volts (we divide by a factor of 2 since the ion gains energy both entering and leaving the buncher). This assumes of course that the buncher is $100 \%$ efficient, and that we operate at the center frequency, $\mathrm{f}_{\mathrm{O}}$, given above.

This brings us to the next question. What is the effect of changing the frequency on the buncher, while the length of the buncher remains constant. It turns out that the buncher has a sinusoidal frequency response with full gain operation only at odd multiples of the of the center frequency. Below we plot the gain of the $90^{\circ}$ ion as a function of the frequency, $\mathrm{f}$, applied to buncher. 


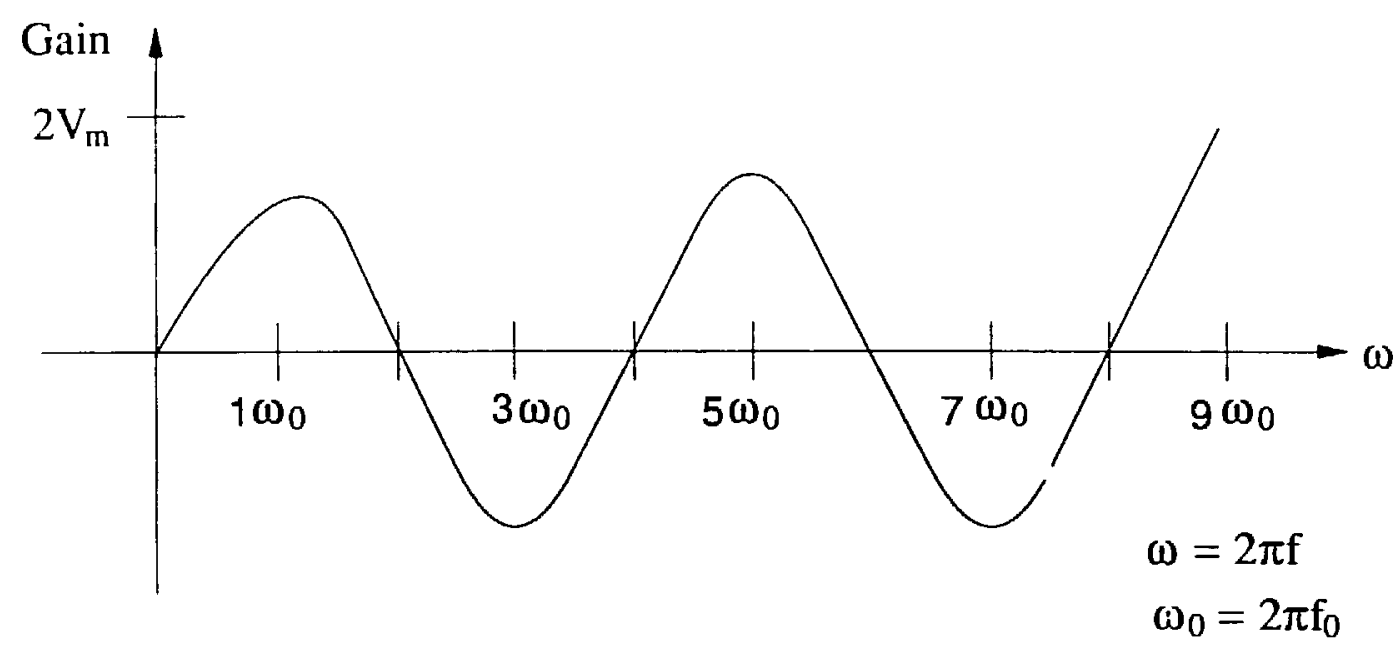

$$
\begin{aligned}
& \text { Gain }=2 e V_{m} \sin \frac{\pi f}{2 f_{0}} \\
& V_{m}=\text { voltage on to buncher } \\
& f=\text { frequency applied } \\
& \text { to buncher } \\
& e=\text { charge on electron }
\end{aligned}
$$

There is no gain at even multiples. This is because an integral number of wavelengths will fit into the buncher, and thus whatever an ion gains on the way in is lost on the way out. For the general case of an ion with arbitrary phase, it is not clear at first what happens to the gain of that ion as the frequency is changed. The solution, though, is quite easy to see from the following phasor diagram and the general equation for energy gain of the ion as a function of the time that it enters the buncher.

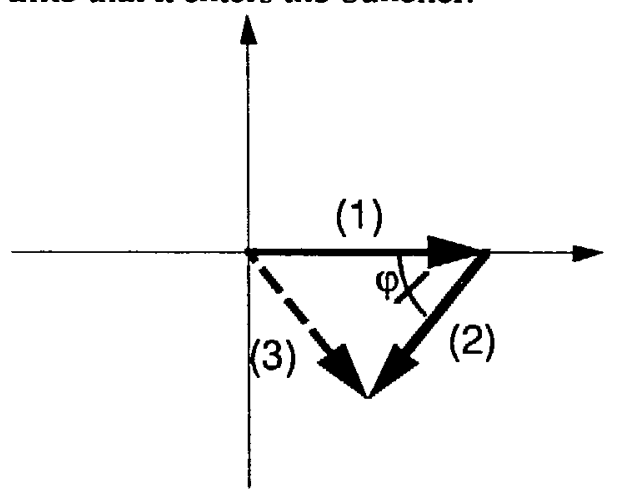

(3)

(1)

(2)

$$
\begin{aligned}
& \text { Gain }=T\left(t_{1}\right)=e V_{m}\left[\sin \left(2 \pi f t_{1}\right)-\sin \left(2 \pi f_{1}+\pi \frac{f}{f_{0}}\right)\right] \\
& t_{1}=\text { time ion enters the buncher } \\
& \varphi=\pi f / f_{0}=\text { phase angle }
\end{aligned}
$$


As you can see, changing the frequency implies a rotation of the second phasor in the diagram, which changes the magnitude of the gain. If $f$ is an odd multiple of $f_{0}$ then (1) and (2) add for maximum amplitude. When $f$ is an even multiple of $f_{0},(1)$ and (2) cancel. To get the time dependence we recall that all the phasors have the same time dependence $=$ $2 \pi \mathrm{ft}]$. This means that even though we are not operating at the center frequency, the ions will still have a gain which varies sinusoidaly with the time they enter the buncher, however the magnitude of the gain will be reduced unless you happen to be operating at exactly a odd harmonic of the center frequency.

It would appear from the above equations and plots, that the voltage needed to bunch ions would be a symmetric function about the center frequency. This though is not the case. The above equations give the amount of energy gained by the ion, not the energy required to bunch the ions. One has to take into account the fact that as the frequency is changed, the actual distance to be bunched, $d$, is also changed. $d$ is still taken to ' a $1 / 4$ of a wavelength, but now that wavelength corresponds to a new frequency, f. Below we give the expression for the magnitude of the applied voltage necessary to bunch ions at arbitrary frequency and center frequency.

$$
V_{m}=\frac{\pi T_{0} \sqrt{\frac{2 T_{0}}{M}}}{8 l_{c} e f_{0}}\left[\frac{1}{\left(\frac{\pi f}{2 f_{0}}\right) \sin \left(\frac{\pi f}{2 f_{0}}\right)}\right]
$$

In figure F.2 we give a plot of the function: $\quad \frac{1}{X \sin X}$

Notice that one has to be carefui in picking the center frequency since the function tends to blow up for $\mathrm{X}<1$ or $\mathrm{X}>2.75$. This implies for the two choices of center frequency, 9 and $11 \mathrm{MHz}$ :

center frequency

$9 \mathrm{MHz}$

$11 \mathrm{MHz}$ operating range

5.4 to $16 \mathrm{MHz}$

6.6 to $19 \mathrm{MHz}$

We will be operating mostly at the higher harmonics so we go with the $11 \mathrm{MHz}$ design.

One may also ask what is the effect of changing the mass of the ion. The above equation still holds, but now the center frequency has to be replaced by an effective center frequency given by the following equation. Also the mass, $M$, has to be replaced by the new mass, $\mathrm{M}^{\prime}$.

$$
f_{\text {Oeff }}=f_{0} \sqrt{\frac{M}{M^{\prime}}}
$$


This means that as we lower the mass, the buncher corresponds to a higher center frequency. This effect, though, is canceled in our machine because we change the resonant frequency as the mass changes in order to keep the cyclotron radius (in the magnetic field) constant ( $f * M^{.5}=$ constant) This also the cancel the other $M^{-.5}$ in the formula for $V_{m}$ given above. Thus the mass does not change the voltage necessary to bunch the ions.

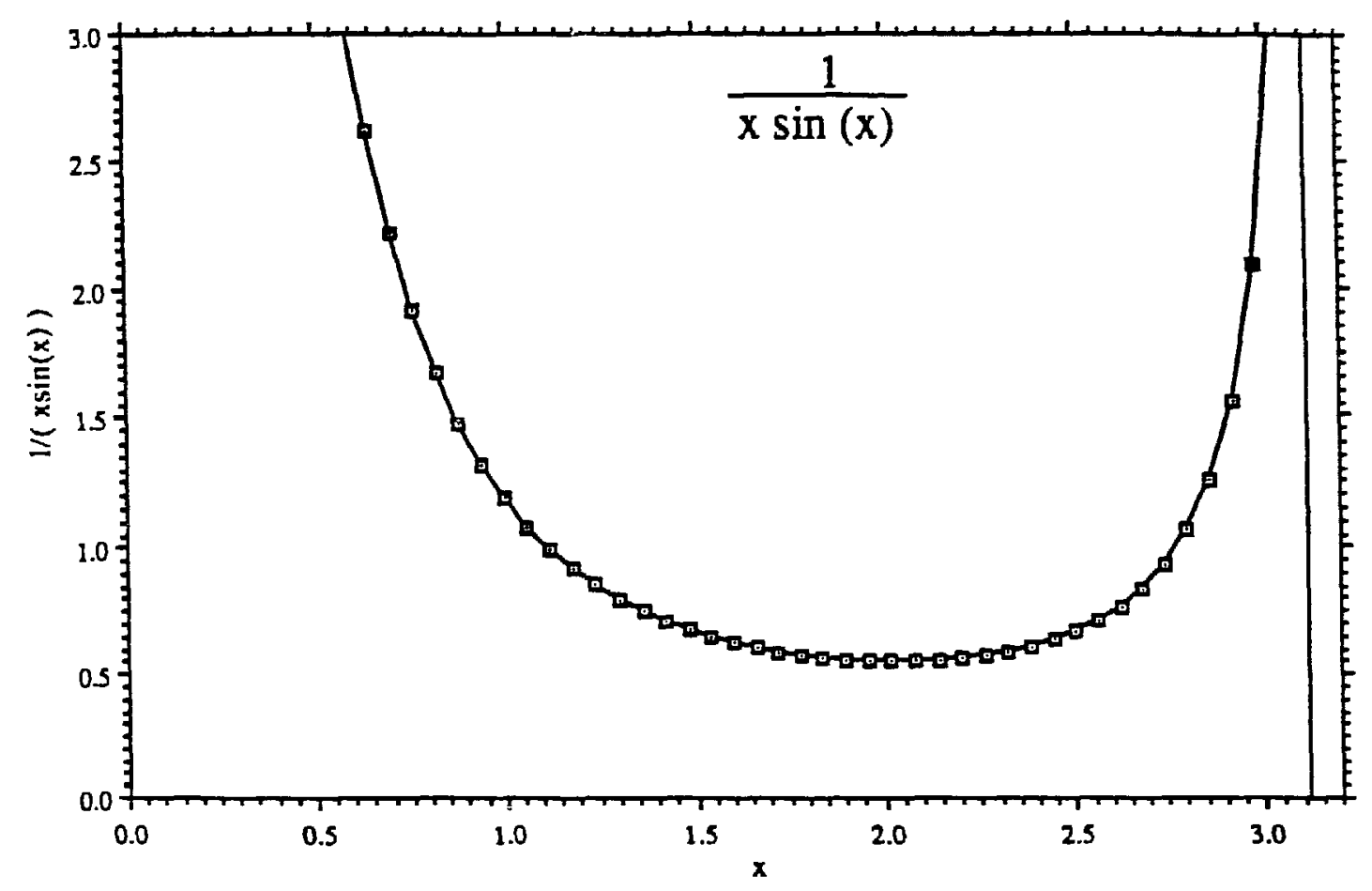

Figure F.2 Plot of the function $\frac{1}{X \sin X}$

\section{F.3.1.2 Effect of Finite Gaps and Grids on Beam Quality:}

Now we turn to a more realistic analysis of a simple buncher. Previously we assumed that the gaps between the three electrodes in the simple buncher were infinitely small. In reality the gaps are of finite width, so we must decide how large to make them. To understand the effect of a finite gap, we first look at the simple case of two parallel plates, one of which is grounded while the other is driven by a sinusoidal voltage, $V(t)=$ $V_{m} \sin (\omega t)$. The ratio of the kinetic energy gain (T) of an ion to the maximum kinetic energy gain $\left(T_{\max } @ g=0\right)$ is given by the sinc function. 


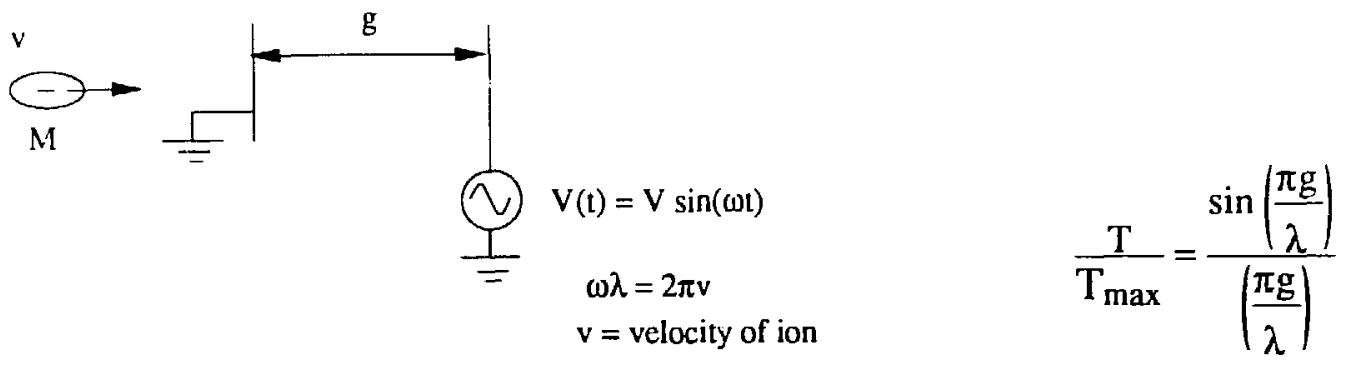

This effect is often times referred to as the transit time effect [5] If $g=\lambda / 4$ then the gain only drops by about $10 \%$.

In the buncher we not only have finite gaps, but we also have grids rather than parallel plates. The grids accelerate the ions through the buncher. It was decided that an analysis should be done on the effect of grids on the beam quality and voltage efficiency of the buncher. The important factor here is beam quality, ie. uniformity in gain for ions traveling in the same transverse plane, and minimum transverse dispersive effects. The grid problem was solved in two steps. First we considered the electrostatic case and looked at different aspect ratios of grid spacing to gap spacing. This allowed for a qualitative understanding of the problem. Next we considered the time varying problem for two specific aspect ratios, and integrated the forces to yield the energy gain in the axial and transverse directions for a variety of wavelengths. This problem has also been discussed by other authors $[5,7]$ in varying degrees of detail and generality.

The first step in solving this problem involved solving Laplace's equation for an infinite grid. This was done using conformal mapping methods [8]. Please note that now y is the axial direction while $\mathrm{x}$ is the transverse direction.

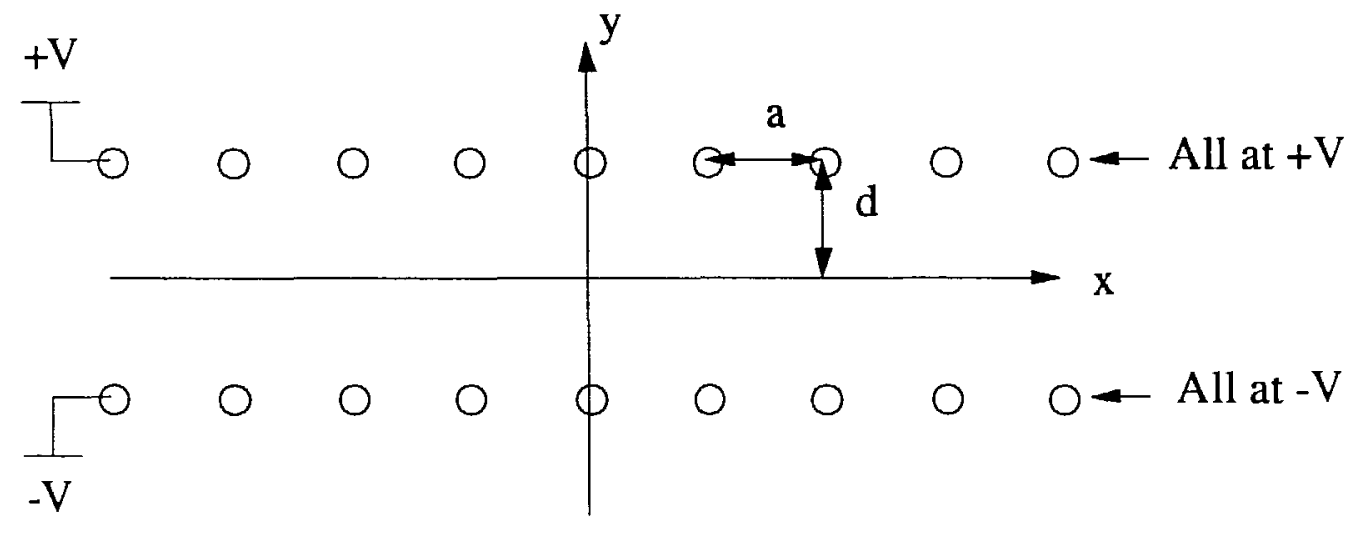

$$
\begin{aligned}
& \Phi(x, y)=K \ln \left[\frac{\cos ^{2} X \sin ^{2} X+(\cosh Y \sinh Y+b \tanh D)^{2}}{\cos ^{2} X \sin ^{2} X+(\cosh Y \sinh Y-b \tanh D)^{2}}\right] \\
& b=\sin ^{2} X \sinh ^{2} Y+\cos ^{2} X \cosh ^{2} Y
\end{aligned}
$$




$$
X=\frac{\pi x}{a} \quad Y=\frac{\pi y}{a} \quad D=\frac{\pi d}{a}
$$

Find $\mathrm{K}$ by using the boundary conditions on the wires.

$\Phi(0, d-r)=+V \quad(r=$ radius of wire $)$

The above equation for $\Phi$ can be plotted on a computer. In figure F. 3 we show a plot of the equipotentials. In figures F.4 and F.5 we show the potential profiles as two different values of $\mathrm{x}$ and different aspect ratios. These plots, although not useful for quantitative numbers, are extremely useful for a qualitative feel for the effects of grids on the beam quality. You will notice right away from Fig. F.3 that an ion traveling in the $y$ direction at $x=0$ (\#1) will see a radically different potential than than an ion traveling at $x=a / 2$ (\#2). \#1 wiil see an effective gap which is smaller than \#2, therefore \#1 will have an energy gain greater than \#2 due to the transit time effect discussed earlier. For good beam quality, we would like an energy gain variation of less than $10 \%$. In figures F.4 and F.5, we show the potential profile in the $y$ direction at $x=0$ and $x=a / 2$ for two different wire aspect ratios, $a=d$ and $a=2 d$, with $d$ held constant. As intuition might suggest, $a=d$ gives a potential curve which is more linear than $a=2 d$. Also the overall gain is greater in the former case since the potential rises to a higher value as y approaches infinity. This introduces the curious point that although the potential on the wire is one volt, the potential at infinity is not one volt but rather 0.7 volts for the $a=d$ case and 0.5 volts for the $a=2 d$ case. It appears that there will be a loss in gain due the the transit time effect and due to the fact that we do not have an infinite number of grids. It is not clear that the second effect will be observed experimentally, since we have solved the problem for an infinite grid isolated in space (other conductors will probably change the spatial variations in the potential). This drop in gain though is significant, and should be taken into consideration in the design of the RF electronics. 

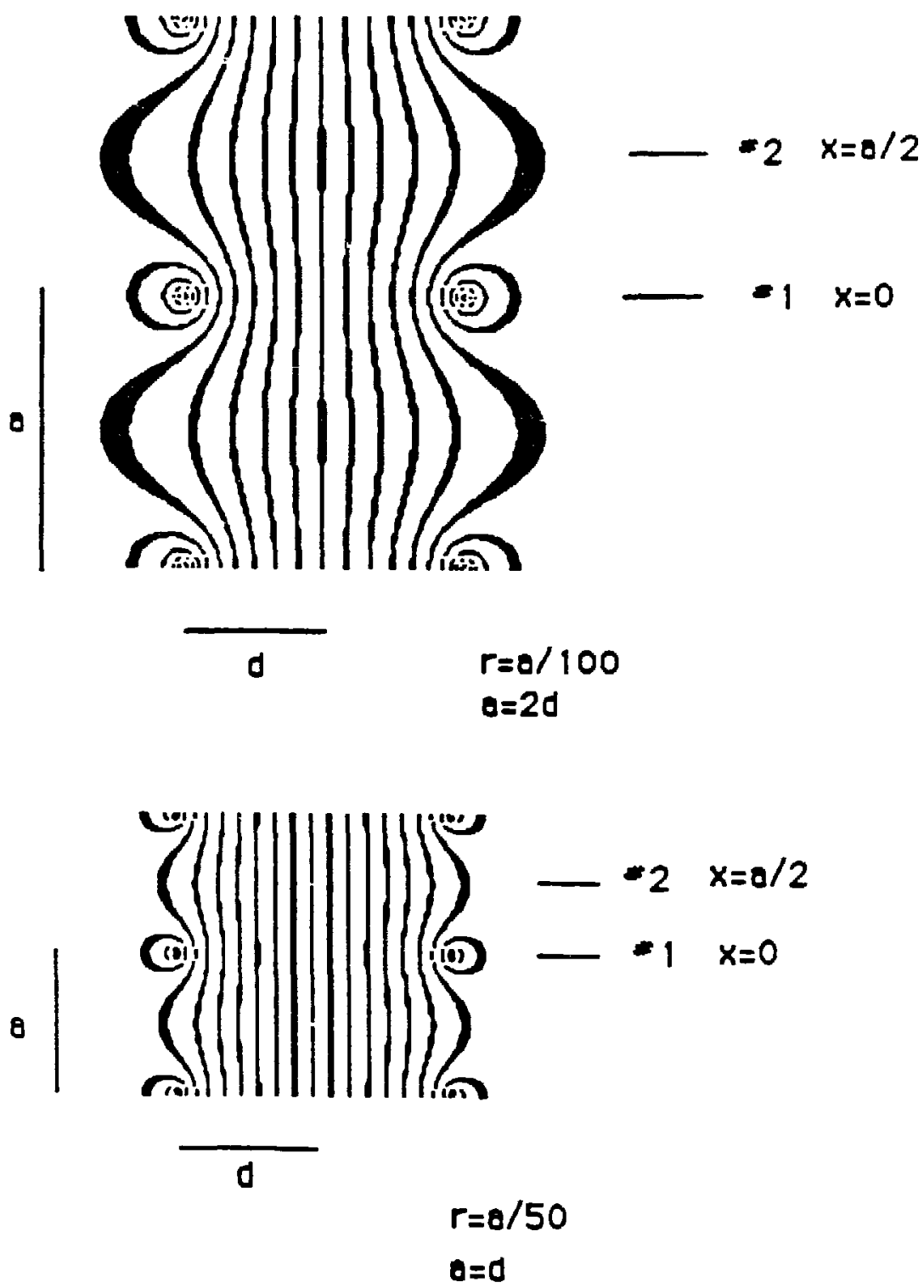

Figure F.3 Equipotentials near grids. Equipotential separation $=0.1$ 


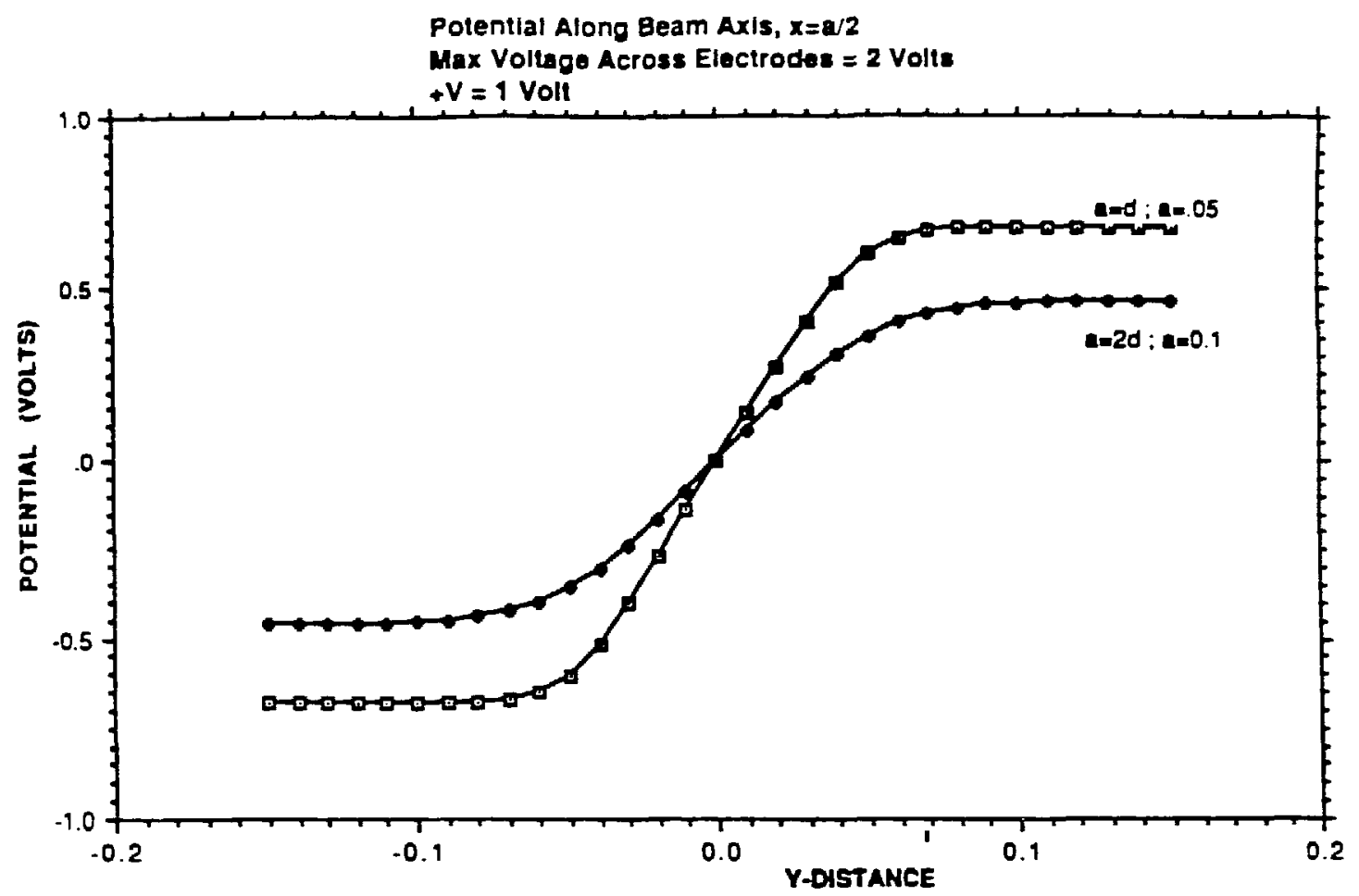

Fig. 4

Figure F.4

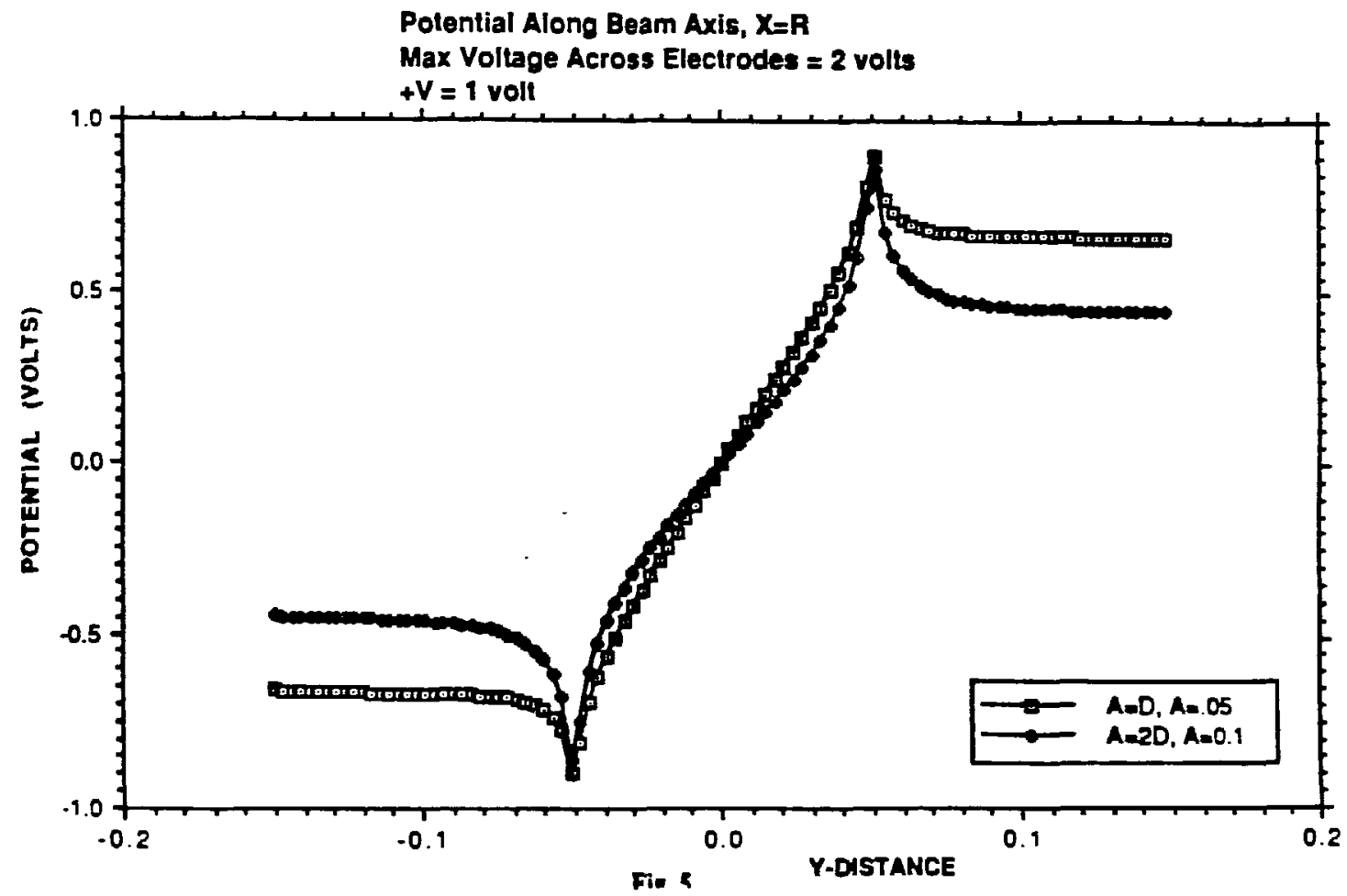

Figure F.5 
We now turn to the real problem we would like to solve, axial gain and transverse gain in a time varying potential. We assume to first order that the path of the ion and its velocity in the axial direction are not affected by the action of the buncher. In the subsequent plots the voltage,

$$
V(t)=V_{m} \sin (\omega \tau), V_{m}=2 \text { volts, }
$$

is applied to one of the grids, while the other grid is grounded (in other words, if we had an infinite number of wires and zero gap, the maximum net gain would be $2 \mathrm{eV}$ ). For each configuration, there are sets of curves corresponding to different wavelengths, where:

$$
\begin{aligned}
& \omega \lambda=v 2 \pi \\
& v=\text { velocity of ion }
\end{aligned}
$$

First we consider the gain in the axial direction for $a=2 d$ (Fig. F.6) and $a=d$ (Fig. F.7), $d=$ constant. The units of distance are arbitrary since the important quantities are the ratios, $a / d$ and $a / \lambda$. We compute the gain for the ion that is at the center of the gap, when the voltage is a maximum (ie. the 90 degree ion discussed earlier). Since the voltage is changing the slowest at this point of the curve, this ion will receive the most axial gain as compared to other ions of different phase.

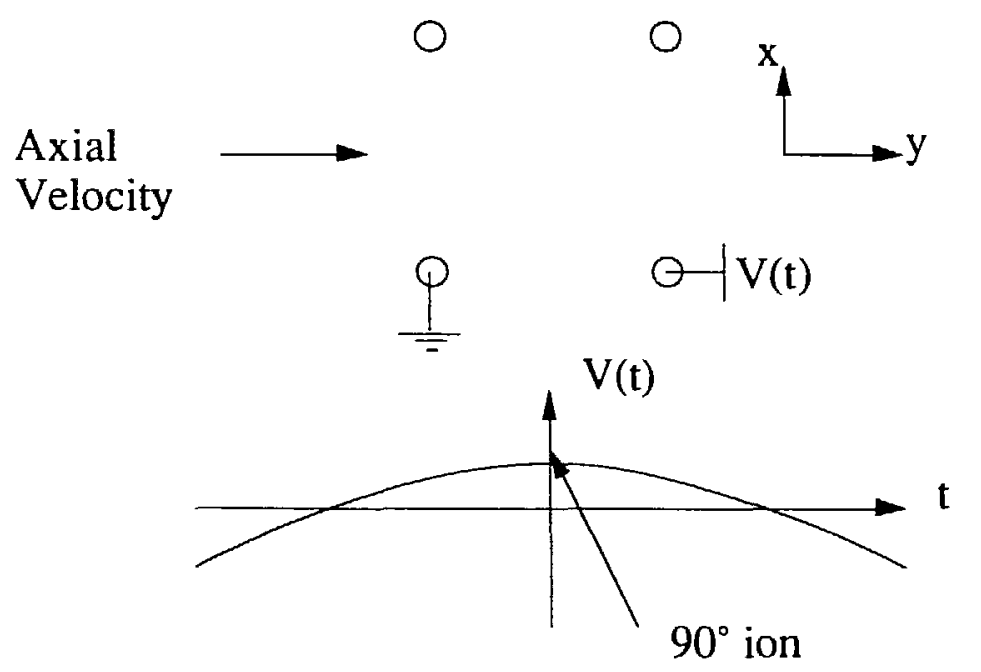

As expected the gain is much more uniform for $a=d$ than $a=2 d$. Also the overall gain is higher in the $a=d$ case. The price though that you pay is that you need twice as many wires, which doubles the amount of beam intercepted by the grid. You will notice that as the wavelength decreases, the gain drops slowly at first, till a threshold is reached, and then the gain drops suddenly. This is the action of the sinc function in the transit time effect discussed earlier. From these two plots, it appears that the $a=d: a=.05$ case, with $\lambda=0.4$ 
is best for our purpose. The gain varies by less than $10 \%$, and the overall gain is $60 \%$ of the ideal case.

AXIAL GAN FOR DIFFERENT WA VELENGTHS

$A=1, D=.05$, WA VELENGHTS GIVEN IN LEGEND

MAX VOLTAGE ACROSS ELECTRODES $=2$ VOLTS

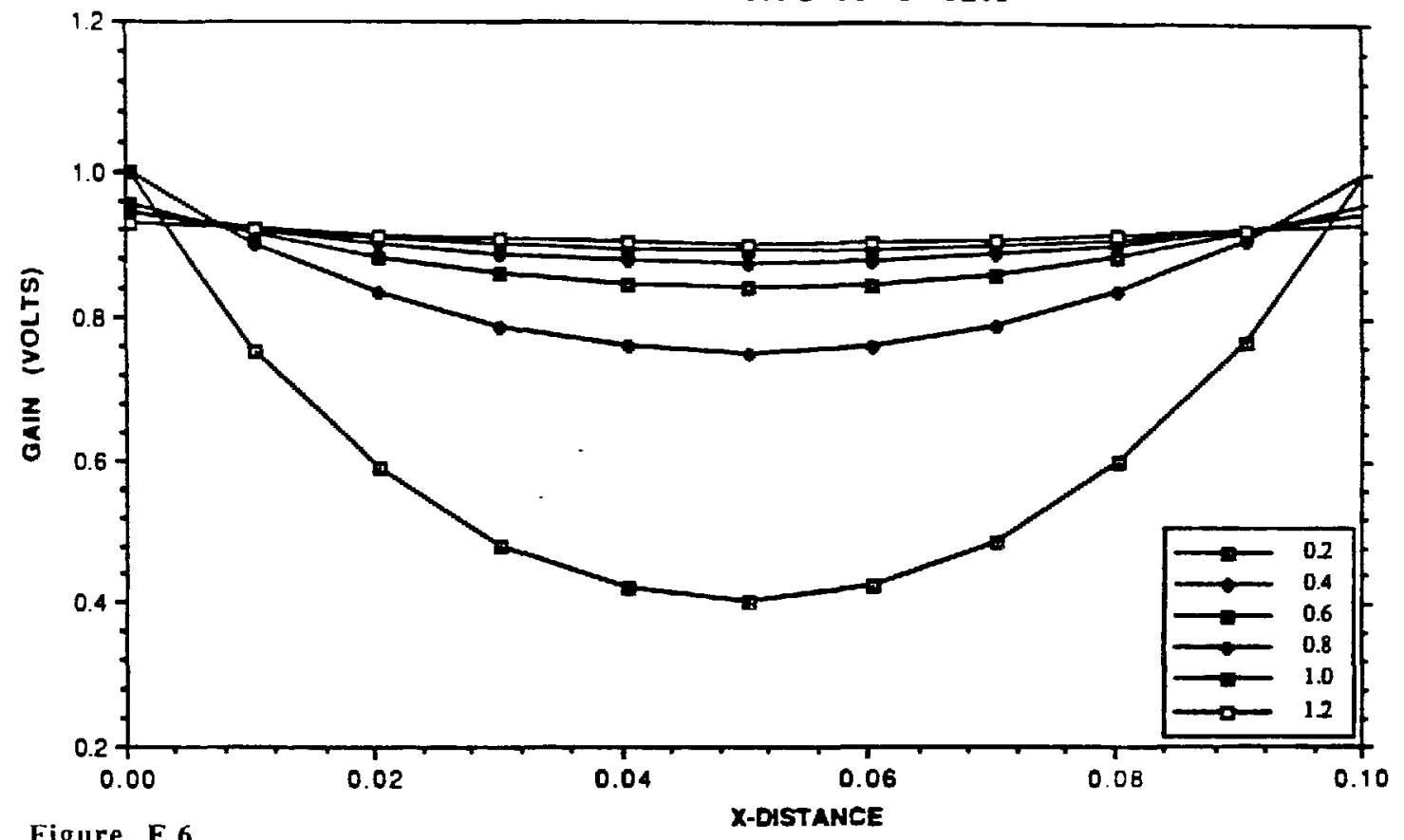

Figure F.6

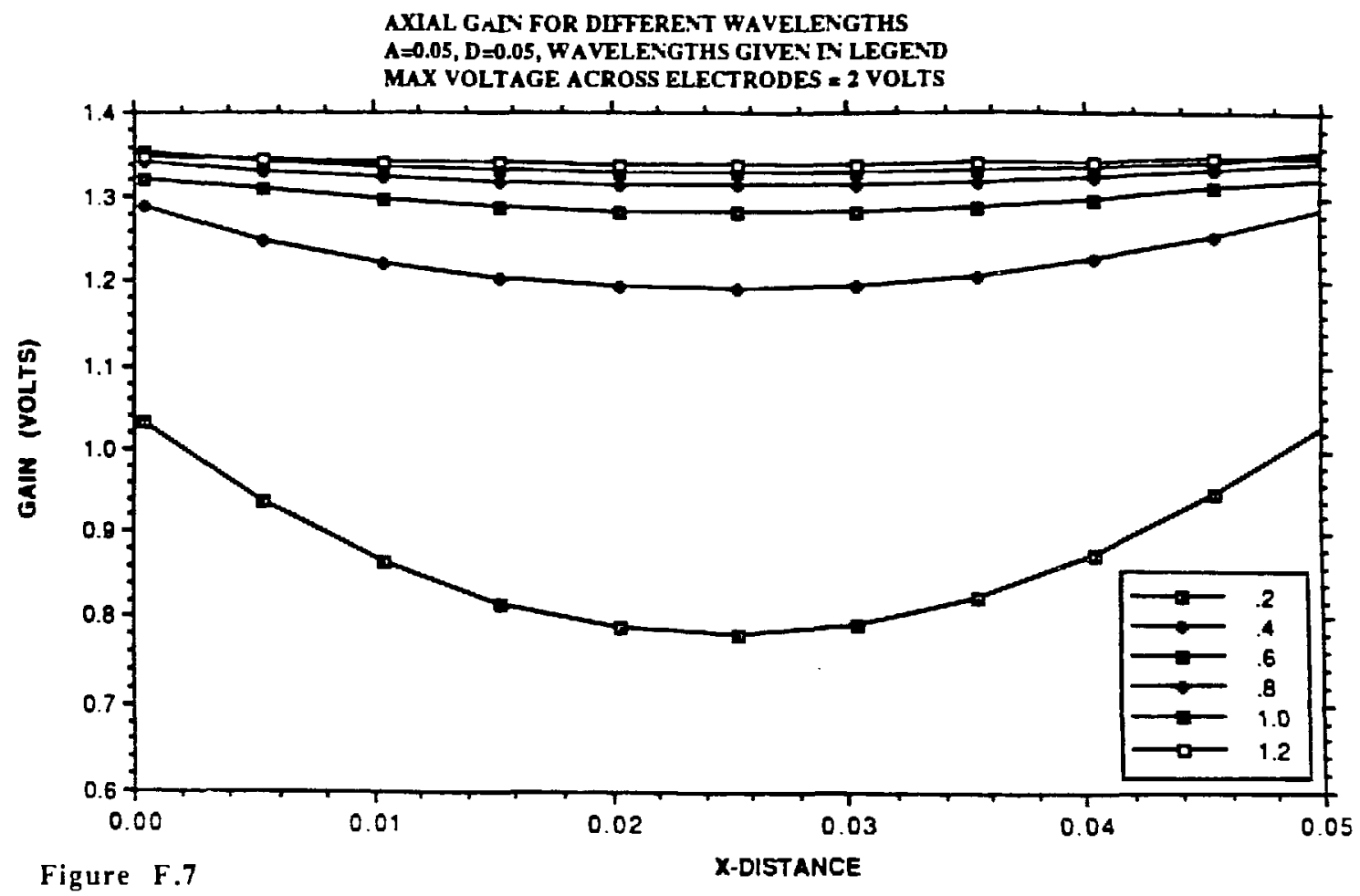


Next we turn to the transverse gain, to see what sort of angular dispersive effects the grids will have on the beam. We compute the gain for the ion that is at the center of the gap, when the voltage is zero (ie. the $0^{\circ}$ ion discussed earlier). Here the voltage is changing the most rapidly, so that there will be a maximum amount of gain in the transverse direction. Notice the important point that the ion that receives the most unansverse gain will also receive the least axial gain. The axial gain is a minimum for the $0^{\circ}$ ion because he sees a force which integrates to zero.

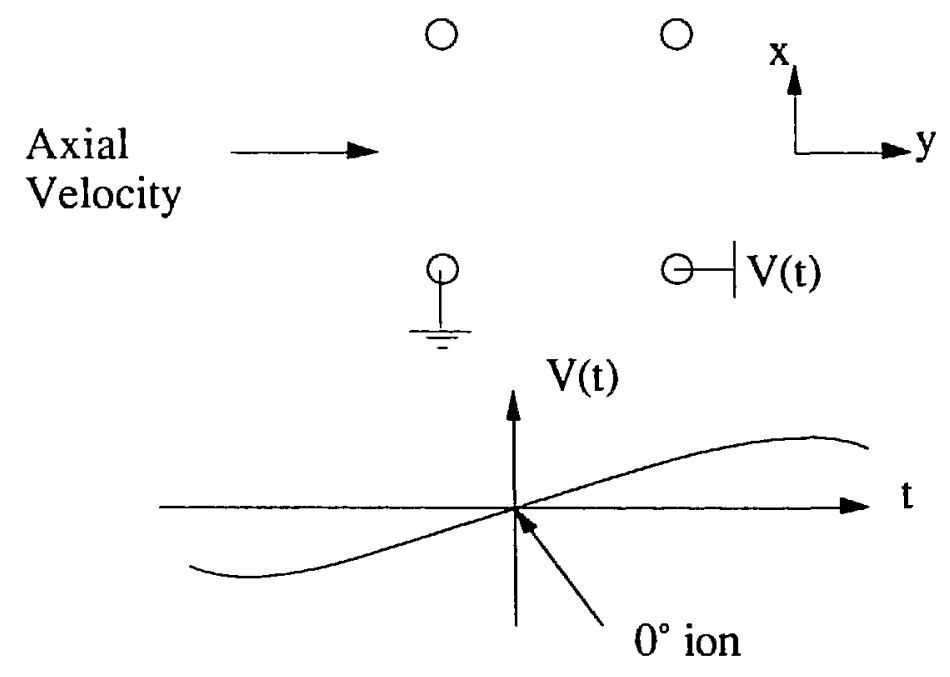

Figures F.8 and F.9 show the transverse gain for the different aspect ratios. For $a=d$, $\mathrm{a}=0.05$, and $\lambda=0.4$, the maximum transverse gain is $0.5 \mathrm{eV}$ which is $1 / 4$ the maximum axial energy gain. We now compute the half angle associated with this transverse gain for the specific example:

$$
\begin{array}{ll}
\mathrm{m}=\text { mass } 14 & \mathrm{a}=\mathrm{d} \\
\mathrm{f}_{\mathrm{O}}=11 \mathrm{MHz} & \mathrm{a}=.05 \\
\mathrm{f}=15 \mathrm{MHz} & \lambda=.4 \\
\mathrm{~T}_{\mathrm{O}}=5 \mathrm{KeV} & \mathrm{a} / \lambda=1 / 8 \\
\mathrm{l}_{\mathrm{C}}=100 \mathrm{~cm} & \\
\mathrm{~V}_{\mathrm{m}} \approx 40 \text { volts = voltage applied to buncher = maximum axial }
\end{array}
$$

energy gain

$$
\begin{aligned}
& \Delta v_{X}=\text { transverse velocity } \\
& v=\text { axial velocity. }
\end{aligned}
$$$$
\text { Half Angle }=B=\Delta v_{x} / v=\Delta T / 2 T
$$ 


$$
\begin{aligned}
& \Delta \mathrm{T}=\mathrm{eV}^{\prime} \mathrm{m} / \mathrm{A}=10 \mathrm{eV} \\
& B=1 \mathrm{mrad}
\end{aligned}
$$

So with the given configuration, we expect mrad of dispersion / gap. The dispersion of the beam at this point in the cyclotron is about $10 \mathrm{mrad}$, so the dispersion caused by the buncher is not excessive.

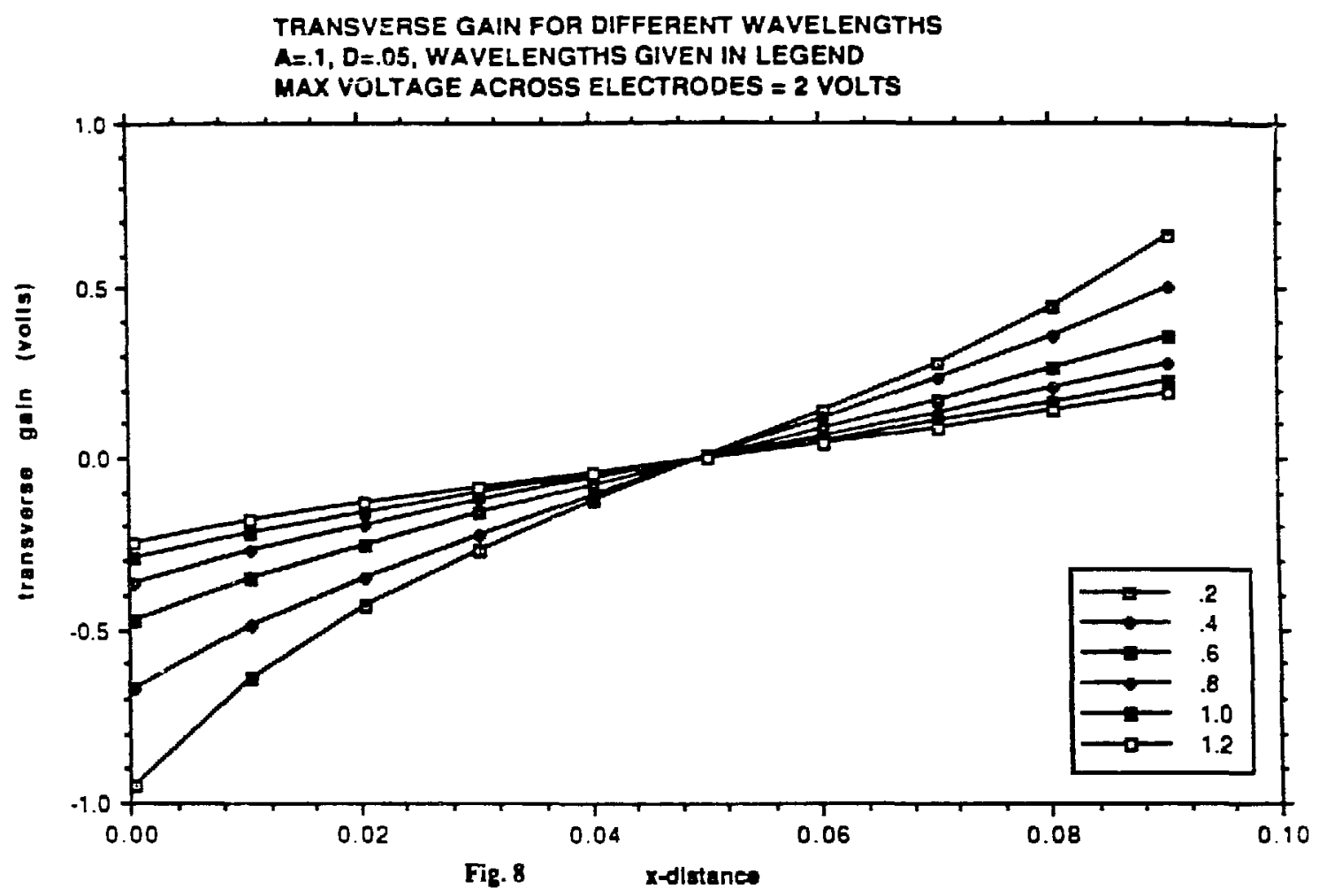

Figure F.8 


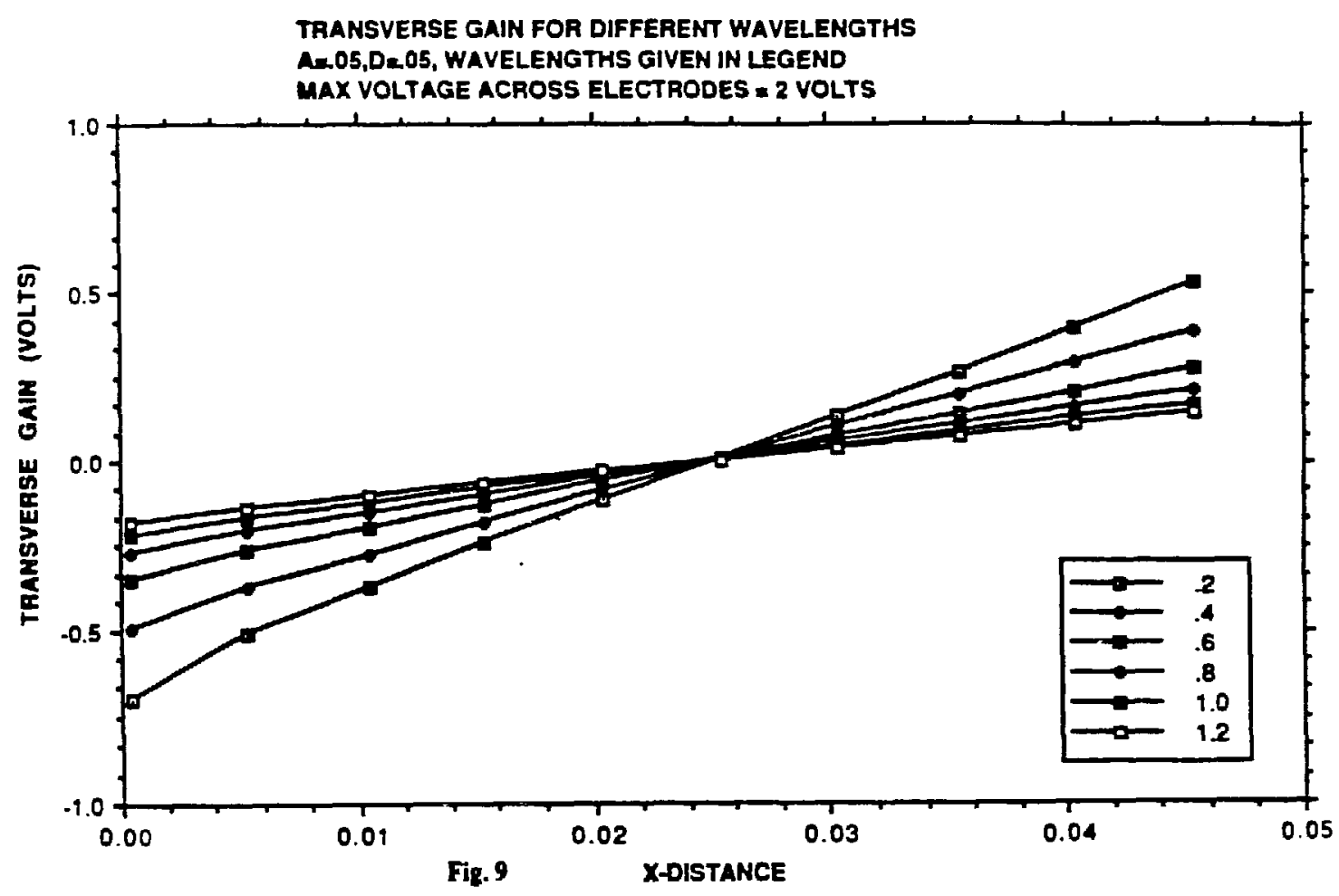

Figure F.9

In the actual buncher, you will recall that there are two sets of grids (entrance/exit). A question arises, as to how to align the second set of grids relative to the first set. There are two choices, perfectly aligned grids or grids $90^{\circ}$ out of phase (of course there are an infinite number of choices but these are the most obvious). It would appear at first that all the wires should be perfectly aligned, so that the four grids appear to be one grid, thereby intercepting less of the beam. This though is difficult to do in practice, so we decided to look into the possibility of non aligned grid wires.

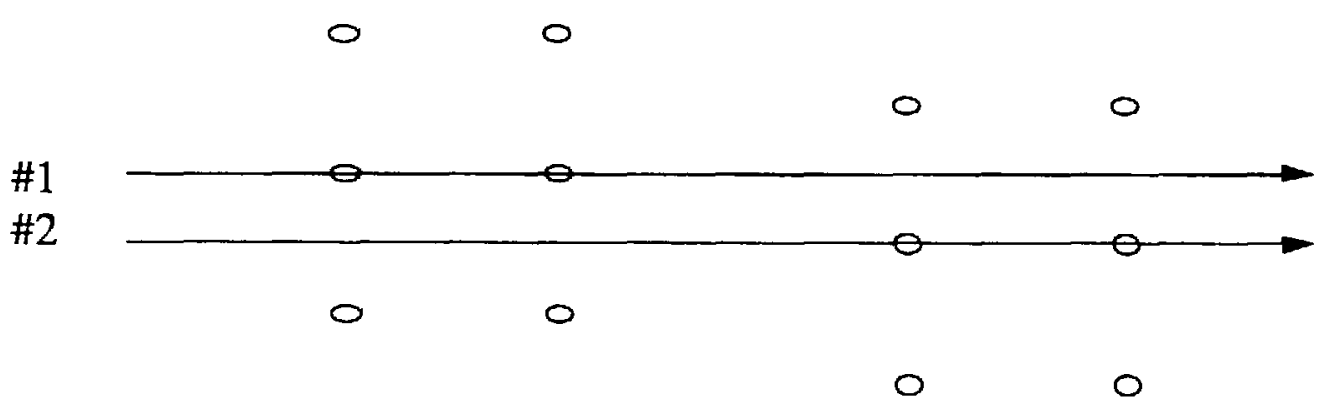

Grid A

Grid B 
As you recall from the previous plots and discussion, ion $\# 2$ will receive less axial gain than ion \#l as they pass through the first set of grids, A, but when they travel through B, the reverse occurs, thereby effectively canceling the nonuniform axial gain introduced at grid A. A similar effect occurs when one considers the transverse gain. Ion \#2 will only receive a transverse gain at grid $B$, while ion $\# 1$ will only receive a transverse gain at grid A. Therefore the ions traveling through nonaligned grids receive half the dispersive effect as compared to the aligned situation. These two factors are a great advantage since it should allow for a reduction in the number of grid wires without a severe degradation in beam quality. The disadvantage, of course, is that in the ideal case twice as many ions will be intercepted by the nonaligned method than the aligned method. In practice, though, alignment is difficult, and rarely can one align all four grids perfectly.

This then concludes a fairly involved discussion of the dynamic properties of a simple buncher.

\section{F.3.1.3 Other Effects:}

Now we turn to some final considerations on the physical design. One important point is the orientation of the grid wires relative to the beam. We show below a sketch of the beam profile, and

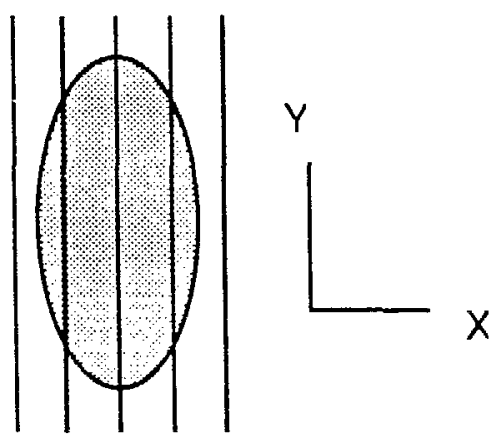

its orientation with respect to the grid wires. The major axis of the beam should lie along the direction of the wires. This has two advantages, (1) a minimum amount of the beam is intercepted by the wires,and (2) the transverse energy goes into the $\mathrm{x}$-direction of the beam which already has the larger divergence (this assumes the beam is nearly focused at the buncher). The last important point to consider is that the beam, as it passes through the buncher, should not see any insulators such as ceramic. The problem is that the insulators can become charged up and the deflect the beam which may in turn cause discharging. This generally leads to instabilities which are hard to diagnose and generally wreak havoc with the entire experiment. 


\section{F.3.1.4 Final Design Parameters:}

This completes all the considerations necessary to properly design the mechanical components of a simple buncher. We will conclude this section with a sketch of the buncher, along with the necessary parameters to build one.

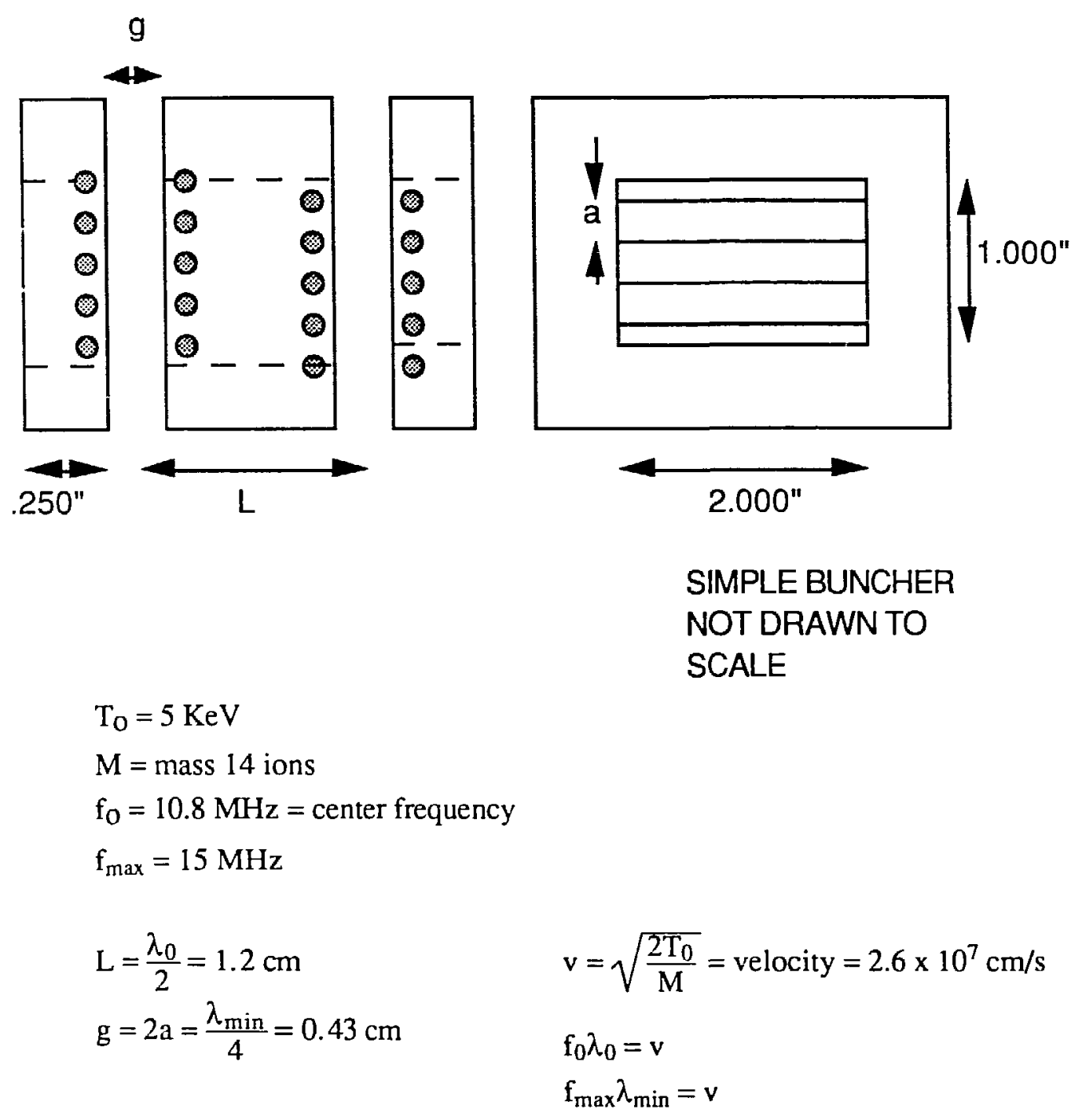

\section{F.3.2 Electronics Design:}

With the mechanical design completed we now turn briefly to the necessary electronics for the buncher. As stated previously, much of the equipment necessary can be 
bought off the shelf [9] or salvaged from around the laboratory. Here are the basic design recpuirements for the buncher electronics.

1. Frequency range, $5 \mathrm{MHz}$ to $17 \mathrm{MHz}$

2. Voltage range, $0-200$ volts

3. Phase shift, $0^{\circ}$ to $360^{\circ}$

4. Power consumption $<100 \mathrm{Watts}$

5. RF Signal Source the same as for the cyclotron

First we give the reason for voltage requirement. In the ideal case at $11 \mathrm{MHz}$, the voltage needed is 30 volts (see sec. F.3.1.1). This should be increased by a the following factors:

$\begin{array}{ll}2 \mathrm{X} & \text { due to frequency variation } \\ 1.5 \mathrm{X} & \text { grids } \\ 2 \mathrm{X} & \text { Safety factor }\end{array}$

This totals to about 180 volts.

Next we discuss the phase shifter. It is necessary to adjust the phase of the buncher relative to the phase of the cyclotron, in order to have the bunches of ions properly accelerated by the RF on the cyclotron. At the moment we have acquired a delay box (essentially coax cable and switches) to act as a phase shifter. It would be desirable in the future to have an electronic phase shifter, which would continuously adjust the phase from $0^{\circ}$ to $360^{\circ}$. An easy way to do this is with some $0^{\circ}-90^{\circ}$ phase splitters and electronically controlled attenuators. Here is a block diagram for a phase shifter $[10,11]$. See [11] for circuit.

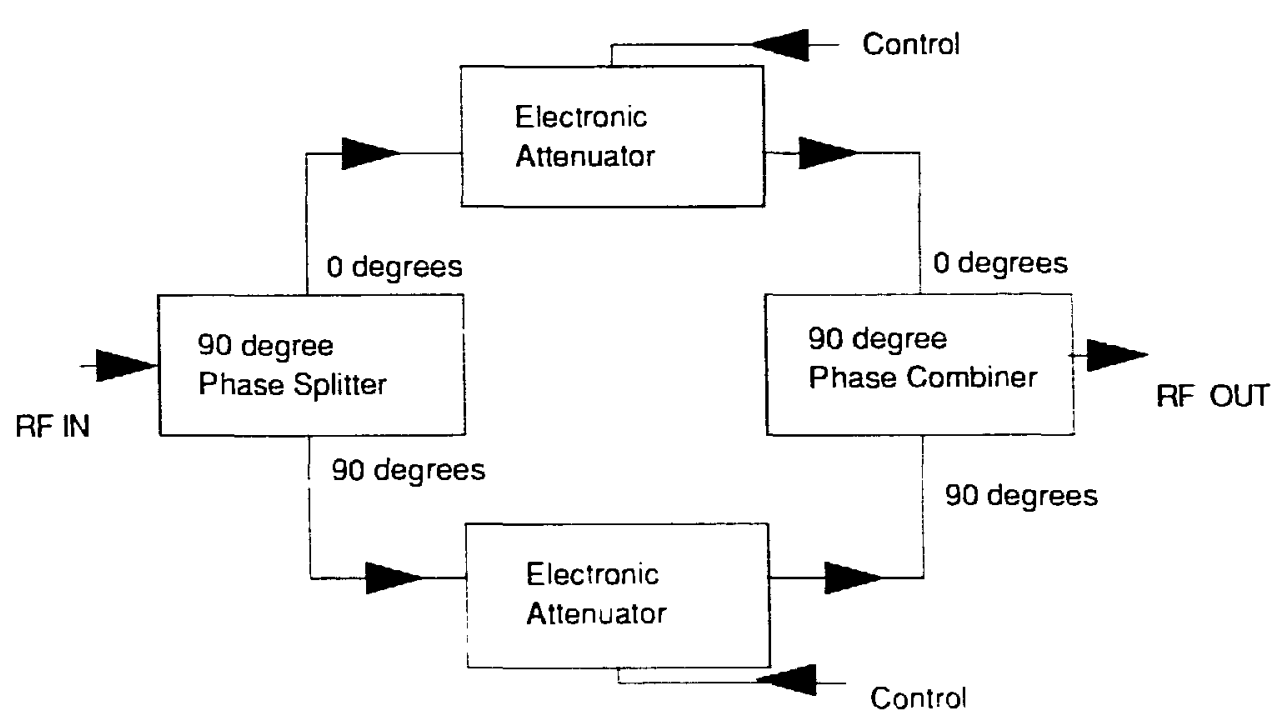


The input signal is first split into two signals which are $90^{\circ}$ out of phase. Each signal is then attenuated by the proper amount and then finally recombined to form a signal shifted from $0^{\circ}$ to $360^{\circ}$. The principle behind this is most easily seen from a phasor diagram, where one signal is along the real axis while the other signal is along the imaginary axis. By combining the signals in the proper ratio, one can cause the resultant sigual to rotate in the complex plane, thus shifting the phase of the output relative to the input.

Next we give a block diagram of the RF electronics for both the cyclotron and the buncher.

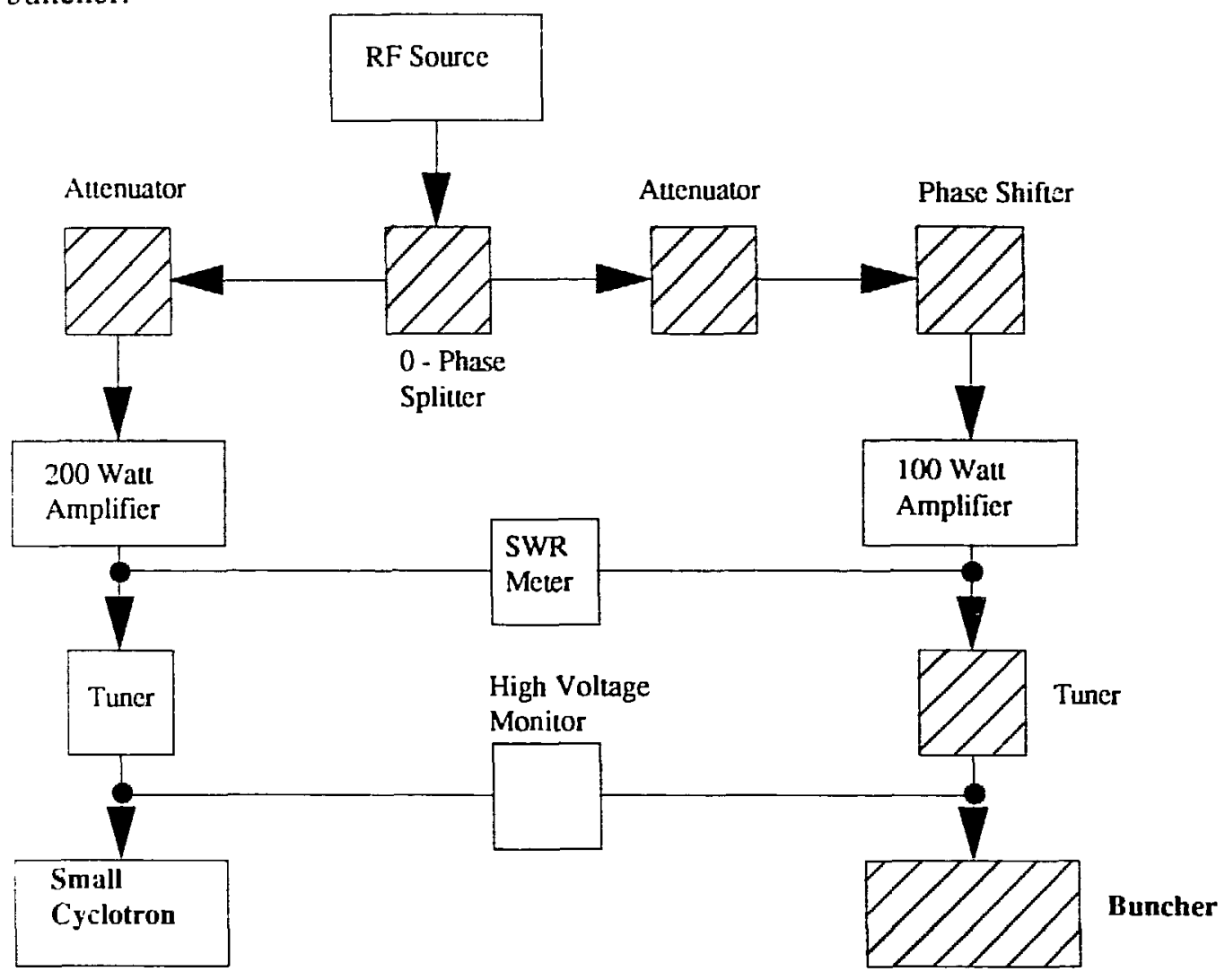

CYCLOTRON - BUNCHER

BE ELECTRONICS

LAYOUT

7 Components need to be purchased or made

Components already exist 


\section{E.4.Conclusions:}

This concludes the design of a simple buncher for use with the Berkeley Small Cyclotron. In summary, the buncher should boost the efficiency of the system by a factor of five which will allow the dating of archaeological samples in a practical amount of time. Also we have discussed a variation on the simple buncher, the gap buncher which employs three grids and a square wave, which allows for perfect linear bunching of $50 \%$ of the beam. Although perfect bunching is not necessary for the small cyclotron, the gap buncher may prove useful in other accelerators.

\section{F.5. References:}

[1] K.J. Bertsche, P.G. Friedman, D.E. Morris, R.A. Muller, J.J. Welch. Nucl. Instr. \& Meth. B29 (1987) 105-109.

[2] J.J. Welch, K.J. Bertsche, P.G. Friedman, D.E. Morris, R.A. Muller, P.P. Tans, Nucl. Instr. and Meth. B 18 (1987) 202-214.

[3] Currently under proposal by Tony Sargent, Research Medicine, LBL.

[4] K.J. Bertsche, PhD Thesis, Appendix G.

[5] F.J. Lynch, R.N. Lewis, L.M. Bollinger, W. Henning, O.D. Despe, Nucl. Instr. and Meth. 159 (1979) 245-263.

[6] W.T. Milner, IEEE Tran. on Nucl. Sci., Vol. NS-26, No. 1, February 1979.

[7] A. Septier, Focusing of Charged Particles, Academic Press Inc., New York, New York, 1967.

[8] Solution carried out by K.J. Bertsche

[9] Suppliers, Mini-Circuits, Wideband Engineering, Kay Electronics, and Pasternack.

[10] Private communications with Bob Lamb.

[11] G. Perica, Mini-Circuits Applications Notes, AN1. (1987-88) 6-7.

\section{F,6. Acknowledgements:}

I would like to thank the following people for their support in this project.

Professor Muller for allowing me to use his facilities and mostly for showing me with actions rather than words that a physicist can wear many hats.

Kirk Bertsche for many helpful and long discussions on cyclotrons and RF electronics. Especially helpful were his physical insights into the many different electrode configurations for bunchers. 
Gabriel Paulson for his help in wrestling with the physics of bunchers.

Dave Clark for discussions on the physics of bunchers and their mechanical design. Also helpful were the papers that he sent me describing different bunching systems.

Bob Lamb for his ideas on the RF electronics.

And mostly to Stephanie Karadi and Little Miyo for their endless support and smiling faces, without whom much of my drive to study physics would be extinguished. 


\section{Appendix G: Counting Rates and Sample Sizes}

\section{G. 1 Errors}

The fundamental equation for isotopic decay is (Faure, 1977):

$$
-\frac{d N}{d t} \propto \lambda N
$$

where $N$ is the number of atoms of the radioisotope at a time $t$. The number of isotopes decaying in a fixed time varies linearly with the total number of isotopes present. The decay constant $\lambda$ is related to the half life $\tau_{1 / 2}$ :

$$
\lambda=\frac{\ln 2}{\tau_{1 / 2}}
$$

Equation (G.1) may be re-expressed as:

$$
\frac{d N}{N} \propto-\lambda d t
$$

showing that the fractional change in $\mathrm{N}$ is a linear function of time. This equation may also be used, however, in a dating context to relate the fractional error in measuring the number of decays to the associated error in the date. Interpreting (G.3) in this way, we find the interesting result that a given fractional measurement error translates into a fixed time error, independent of sample age. For ${ }^{14} \mathrm{C}$, with $\tau_{1 / 2}=5730$ years, we firid:

Table G.1

Errors in Radioisotope Measurements

$\begin{array}{ccc}\text { Measurement Error } & \text { Date Error } & \text { Counts Needed } \\ 10 \% & 827 \mathrm{yrs} & 100 \\ 3 \% & 248 \mathrm{yrs} & 1000 \\ 1 \% & 83 \mathrm{yrs} & 10,000 \\ 0.3 \% & 25 \mathrm{yrs} & 100,000\end{array}$

The precision of measurement which is necessary is thus determined by the required precision of age determination. Assuming that systematic enrors are eliminated. the remaining measurement errors are primarily due to counting statistics. An exponential 
decay results in a Poisson distribution of counts, with an RMS error of $\sqrt{N}$ for large $N$. The number of counts needed is shown in Table G.l.

\section{G.2 Counting Rates}

The number of atoms counted depends on the number of atoms available and on the efficiency of the measurement process.

$$
N_{\text {actual }}=\eta N_{\text {available }}
$$

Of the available atoms, only a fraction of them will be ionized and exit the ion source as countable ions. The rest will be lost in the ion source or will exit as molecular ions or as ions of incorrect charge state. It will be convenient to write efficiency, $\eta$, as the product of two terms:

$$
\eta=\eta_{\text {source }} \eta_{\text {accelcrator }}
$$

where $\eta_{\text {source }}$ is the efficiency of correctly ionizing sample atoms and $\eta_{\text {accelerator }}$ is the efficiency of the rest of the system. Hence:

$$
N_{\text {ionized }}=\eta_{\text {source }} N_{\text {available }}
$$

But $N_{\text {ionized }}$ is related to the output charge $Q$ :

$$
N_{\text {ionized }}=\frac{Q}{e}=\frac{r Q_{\text {tolal }}}{e}
$$

where $r$ is the abundance ratio (fraction of current which is the desired species) and is the electron charge. (It is assumed here that atoms are only singly-ionized.)

Substituting (G.5,6,7) into (G.4):

$$
N_{\text {actual }}=\eta N_{\text {ionized }}=\frac{r Q_{\text {total }}}{e}
$$

The number of ions, $N_{\text {actual }}$, can be converted to count rate, $f_{\text {actual }}$, by dividing by count time:

$$
f_{\text {actual }}=\frac{\eta_{\text {accelerator }} r I}{e}
$$


where $/$ is the total beam current.

For a radioisotope, the abundance ratio $r$ can be expressed as $r_{0}$, the modern abundance, times an exponential factor, following directly from integration of the fundamental equation (G.1):

$$
N=N_{0} e^{-\lambda}, \text { or } r=r_{0} e^{-i s}
$$

If this is substituted into (G.9), the count rate may be expressed in terms of the sample age:

$$
f_{\text {actual }}=\frac{\eta_{\text {accelerator }} I r_{0} e^{-i s}}{e}
$$

The time $T$ required to make a measurement depends on the number of counts needed and the count rate:

$$
T=\frac{N_{\text {actual }}}{f_{\text {acaual }}}=\frac{N_{\text {actual }} e}{\eta_{\text {accelerator }} I r_{0} e^{-\lambda s}}
$$

Thus the results of the previous section do not tell the whole story. Although a given fractional error results in a given time error, independent of the age of the sample, we see that older samples require longer counting times.

For carbon, the modern abundance ratio of ${ }^{14} \mathrm{C} /{ }^{12} \mathrm{C}$ is about $1.2 \times 10^{-12}$. Substituting for this and for electron charge in (G.12):

$$
T=\frac{N_{\text {actual }} 1.3 \times 10^{-7}}{\eta_{\text {accelerator }} I_{\text {carbon }} e^{-\hat{\lambda} s}}
$$

The efficiency of the small cyclotron, $\eta_{\text {accelerator, }}$ is about $5 \times 10^{-5}$ (see section 5.1 ). The General Ionex ion source is capable of producing carbon currents of over $10 \mu \mathrm{A}$. Assuming $10 \mu \mathrm{A}$ output, (G.13) becomes:

$$
T=\frac{(270 \mathrm{sec}) N_{\text {actual }}}{e^{-\bar{\lambda} s}}
$$

Thus one atom is counted about every 5 minutes for modern carbon. As mentioned in the text, this rate is much too low. But it should be possible to increase it by 2 orders of magnitude by making the modifications discussed in the text; then we would see a count every 3 seconds. This would be quite practical; one could make a $3 \%$ measurement on modern material in less than an hour. 


\section{G.3 Sample Sizes}

The number of atoms necessary in the sample is easily calculated:

$$
N=\frac{N_{\text {actual }}}{\eta r}=\frac{N_{\text {actual }}}{\eta_{\text {source }} \eta_{\text {accelerator }} r_{0} e^{-\lambda s}}
$$

Converting this to sample size, we obtain:

$$
m=\frac{Z N}{N_{A}}=\frac{Z N_{\text {actual }}}{\eta_{\text {source }} \eta_{\text {accelerator }} N_{A} r_{0} e^{-\lambda s}}
$$

where $N_{A}$ is Avagadro's number, $6.023 \times 10^{23} \mathrm{~g} / \mathrm{mol}$ and $Z$ is atomic number. The ion source that we used should have an efficiency of roughly $3 \%$. Substituting these values into (G.16), we have for carbon ( $\mathrm{Z}=12$ for the dominant isotope):

$$
m=\frac{\left(1.1 \times 10^{-5} \mathrm{~g}\right) N_{\text {actual }}}{e^{-\lambda}}
$$

Thus about $10 \mu \mathrm{g}$ of $\mathrm{C}$ is needed per count for modern carbon with the assumed parameters. Again, if the efficiency is increased by two orders of magnitude, 100ng would be needed per count. Since the present count rate is about 5 min per count at $10 \mu \mathrm{A}(\mathrm{G} .14)$, we see that the source burns about $2 \mu \mathrm{g}$ of carbon per minute when operating at $10 \mu \mathrm{A}$.

\section{G.4 Tracer Studies}

Thus far, ${ }^{14} \mathrm{C}$ measurements have been discussed in a dating context. If instead, ${ }^{14} \mathrm{C}$ is being used as a tracer, the equations are modified slightly. The abundance ratio is not determined by (G.10), but by the dynamics of the system to be traced, the amount of ${ }^{14} \mathrm{C}$ injected, and any subsequent dilutions. Thus, (G.14) and (G.17) may be written respectively as:

$$
\begin{gathered}
T=(270 \mathrm{sec}) N_{\text {actual }} \frac{r}{r_{0}} \\
m=\left(1.1 \times 10^{-5} \mathrm{~g}\right) N_{\text {actual }} \frac{r}{r_{0}}
\end{gathered}
$$

where $r$ is the abundance ratio of ${ }^{14} \mathrm{C} /{ }^{12} \mathrm{C}$ in the sample, $r_{0}$ is the modern ratio $\left(1.2 \times 10^{-12}\right)$, and the currents and efficiencies are as stated above. 


\section{REFERENCES}

Adams, A., and F.H. Read. 1972. Electrostatic cylinder lenses II: Three element einzel lenses. J. Phys. E 5: 150-155.

Alvarez, L.W. 1981. The early days of accelerator mass spectrometry. Proceedings of the Symposium on Accelerator Mass Spectrometry, Argonne National Laboratory, May 11-13, 1981. Argonne National Laboratory Report ANL/PHY-81-1. 1-15. Also Lawrence Berkeley Laboratory Report LBL-12846 (May 1981).

Alvarez, Luis W., and Robert Cornog. 1939a. He ${ }^{3}$ in helium. Phys. Rev. 56(4): 379.

Alvarez, Luis W., and Robert Cornog. 1939b. Helium and hydrogen of mass 3. Phys. Rev. 56(6): 613.

Banford, A.P. 1966. The Transport of Charged Particle Beams. London: Spon.

Bennett, C.L., R.P. Beukens, M.R. Clover, H.E. Gove, R.B. Liebert, A.E. Litherland, K.H. Purser and W.E. Sondheim. 1977. Radiocarbon dating using electrostatic accelerators: negative ions provide the key. Science 198(4316): 508-510.

Bennett, C.L., R.P. Beukens, M.R. Clover, D.E. Elmore, H.E. Gove, L. Kilius, A.E. Litherland and K.H. Purser. 1978. Radiocarbon dating with electrostatic accelerators: dating of milligram samples. Science 201: 345-346.

Bertsche, K.J., P.G. Friedman, D.E. Morris, R.A. Muller, and J.J. Welch. 1987. Status of the Berkeley small cyclotron AMS project. Nucl. Instr. \& Meth. B29: 105-109. Also Lawrence Berkeley Laboratory Report LBL-23324 (April 1987).

Bertsche, K.J., P.G. Friedman, R.A. Muller, and J.J. Welch. 1988. Current Status of the Berkeley cyclotrino. Presented at the 13th Intl. Radiocarbon Conf., Dubrovnik, Yugoslavia, June 1988. Lawrence Berkeley Laboratory Report LBL-25450.

Chen, Mao-Bai, De-Ming Li, Wen-Zhao Gao and Son-Mao Xu. 1987. Some peculiar features of a minicyclotron as a mass spectrometer for dating. Proceedings of the 11 th International Conference on Cyclotrons and their Applications. (Tokyo, 1987). 
Chen, Mao-Bai, Wen-Zhao Gao and De-Ming Li. 1989a. Design criteria on a minicyclotron as a mass spectrometer for dating. Nucl. Instr. \& Meth. A278: 402408.

Chen, Mao-Bai, De-Ming Li, When-Zhao Gao, Xi-Lin Zhang and Rong-Fa Zhou. 1989b. Adopting a non-sinusoidal wave dee voltage in minicyclotrons as mass spectrometers for dating. Nucl. Instr. \& Meth. A278: 409-416.

Cohen, Bernard L. 1953. The theory of the fixed frequency cyclotron. Rev. Sci. Instr. 24(8): 589-601.

Cohen, R., and J. Rainwater. 1969. Axial electric focussing in a cyclotron. IEEE Trans. Nucl. Sci. NS-16: 426-429.

Dawson, Peter H., editor. 1976. Quadrupole Mass Spectrometry and its applications. New York: Elsevier.

Drummond, I.W. 1984. The ion optics of low-energy ion beams. Vacuum 34(1-2): 5161.

El-Kareh, A.B., and J.C.J. El-Kareh. 1970. Electron Beams, Lenses, and Optics. 2 vols. New York: Academic Press.

Elmore, David, and Fred M. Phillips. 1987. Accelerator mass spectrometry for measurement of long-lived radioisotopes. Science 236: 543-550.

Faure, Gunter. 1977. Principles of Isotope Geology. New York: Wiley.

Fink, D., M. Paul, G. Hollos, S. Theis, S. Vogt, R. Stueck, P. Englert and R. Michel. 1987. Measurements of ${ }^{41} \mathrm{Ca}$ spallation cross sections and ${ }^{41} \mathrm{Ca}$ concentrations in the Grant meteorite by accelerator mass spectrometry. Nucl. Instr. \& Meth. B29: 275280.

Friedman, P.G. 1986. A low background-rate detector for ions in the 5 to $50 \mathrm{keV}$ energy range to be used for radioisotope dating with a small cyclotron. $\mathrm{PhD}$ dissertation. Lawrence Berkeley Laboratory Report LBL-17804. 
Friedman, P.G., K.J. Bertsche, M.C. Michel, D.E. Morris, R.A. Muller, and P.P. Tans. 1988. Low background-rate detector for $40-\mathrm{keV}$ ions using a conversion dynode and a microchannel-plate electron multiplier to reject low-energy ions, electrons, and photons. Rev. Sci. Instr. 59(1): 98-111. Also Lawrence Berkeley Laboratory Report LBL-22676 (Jan. 1987).

Galejs, A., and C.E. Kuyatt. 1978. Focusing and dispersing properties of a stigmatic crossed-field energy analyzer. J. Vac.Sci. Technol. 15(3): 865-867.

General Electric Co. 1984. Chart of the Nuclides. San Jose, CA.

Gove, H.E., A.E. Litherland and K.H. Purser. 1987. Anno decimo session. Nucl. Instr. \& Meth. B29: 437-438.

Gradshteyn, I.S., and I.M. Ryzhik. 1980. Table of Integrals, Series, and Products. Corrected and Enlarged Edition. Translated from the Russian. London: Academic Press.

Harting, E., and F.H. Read. 1976. Electrostatic Lenses. New York: Elsevier.

Jeffries, C.D. 1951. A direct determination of the magnetic moment of the protons in units of the nuclear magneton. Phys. Rev. 81(6): 1040-1055.

Liebmann, G. 1949. Measured properties of strong "unipotential" electron lenses. Proc. Phys.Soc., London 64(4): 213-228.

Lippert, Von W., and W. Pohlit. 1953. Zur Kenntnis der elektronenoptischen Eigenschaften elektrostatischer Linsen II. Optik 10(9): 447-454.

Lippert, Von W. 1955. Über einige Eigenschaften von elektrostatischen Einzellinsen, deren Achsenpotential fast das Kathodenpotential erreicht. Optik 12(4): 173-180.

Livingood, John J. 1961. Cyclic Particle Accelerators. Princeton: Van Nostrand.

Livingood, John J. 1969. The Optics of Dipole Magnets. New York: Academic Press.

Mann, W.B. 1953. The Cyclotron. London: Methuen. 
Meisburger, W.D., and E.H. Jacobsen. 1982. Electrostatic lens of very low spherical aberration. Optik 62(4): 359-366.

Middleton, R. 1977. A survey of negative ions from a cesium sputter source. Nucl. Instr. \& Meth. 144: 373-399.

Milinković, Lj.S., K.M. Subotić and E. Fabrici. 1988. Properties of centered accelerated equilibrium orbits. Nucl. Instr. \& Meth. A273: 87-96.

Moore, John H., Christopher C. Davis, and Michael A. Coplan. 1983. Building Scientific Apparatus. Reading, Massachusetts: Addison-Wesley.

Morris, D.E. 1986. A new method for injecting charged particles across a magnetic field. Nucl. Instr. \& Meth. A248(2,3): 297-300.

Muller, Richard A. 1976. Radioisotope dating with a cyclotron. Lawrence Berkeley Laboratory Report LBL-5399. Also Science 196 (4289): 489-494 (1977).

Muller, Richard A., Luis W. Alvarez, William R. Holley and Edward J. Stephenson. 1977. Quarks with unit charge: a search for anomalous hydrogen. Science 196: 521523.

Muller, Richard A., Pieter P. Tans, Terry S. Mast and James J. Welch. 1981. Mass spectrometry with a very small cyclotron. Proceedings of the Symposium on Accelerator Mass Spectrometry, Argonne National Laboratory, May 11-13, 1981. Argonne National Laboratory Report ANL/PHY-81-1. 342-345. Also Lawrence Berkeley Laboratory Report LBL-12797 (May 1981).

Muller, Richard A. 1987. Submarines, quarks, and radioisotope dating. In Discovering Alvarez, edited by W. Peter Trower. Chicago: University of Chicago Press. 225-228.

Munro, E., and N.D. Wittels. 1977. Calculation of the optical properties of electron lenses with electron-transparent foils. Optik 47(1): 25-38.

Murray, Raymond L., and Lawrence T. Ratner. 1953. Electric fields within cyclotron dees. J. Appl. Phys. 24(1): 67-69.

Nelson, D.E., R.G. Korteling and W.R. Stott. 1977. Carbon-14: direct detection at natural concentrations. Science 198: 507-508. 
Nishiizumi, Kunihiko and James R. Amold. 1981. Measurement of cosmogenic nuclides in extraterrestrial material. Proceedings of the Symposium on Accelerator Mass Spectrometry. Argonne National Laboratory, May 11-13, 1981. Argonne National Laboratory Report ANL/PHY-81-1. 262-276.

Orloff, J., and L.W. Swanson. 1979. An asymmetric electrostatic lens for field-emission microprobe applications. J. Appl. Phys. 50(4): 2494-2501.

Read, F.H. 1969. Calculated properties of electrostatic einzel lenses of three apertures. $J$. Phys. E 2: 679-684.

Reiser, Martin. 1971First-order theory of electrical focusing in cyclotron-type twodimensional lenses with static and time-varying potentials. J. Appl. Phys. 42(11): 4128-4138.

Riddle, G.H.N. 1978. Electrostatic einzel lenses with reduced spherical aberration for use in field-emission guns. J. Vac. Sci. Technol. 15(3): 857-860.

Rose, M.E. 1938. Focusing and maximum energy of ions in the cyclotron. Phys. Rev. 53: $392-407$.

Seliger, R.L. 1972. E×B mass-separator design. J. Appl. Phys. 43(5): 2352-2357.

Septier, A. 1960. Spherical aberration of some rotationally symmetrical electrostatic lenses for large-aperture beams. CERN report CERN 60-39 (Oct. 13, 1960). Translated from French as Lawrence Livermore National Laboratory report UCRLTRANS-11564.

Septier, Albert. 1961. Strong-Focusing Lenses. In Advances in Electronics and Electron Physics, volume XIV. Edited by L. Marton. New York: Academic Press.

Subotić, K., Lj. Milinković, M. Zupancić, M. Stojanović and D. Novković. 1990). Superconducting mini-cyclotrons as AMS instruments. To be published in Radiocarbon 32(1).

Szilagyi, M. 1983. Improvement of electrostatic lenses for ion beam lithography. J. Vac. Sci. Technol. B1(4): 1137-1140. 
Taylor, R.E. 1987. Radiocarbon Dating: an Archaeological Perspective. Orlando: Academic Press.

Thompson, J.J. 1909. Inaugural address of the 79th annual meeting of the British Association. Nature 81: 257.

Tiefenback, Michael G. 1986. Space-charge limits on the transport of ion beams in a long alternating gradient system. PhD thesis. Lawrence Berkeley Laboratory Report LBL22465 .

van der Merwe, J.P. 1981. The minimization of the spherical aberration of symmetrical electrostatic round two-foil lenses. J. Appl. Phys. 52(3): 1179-1183.

van Gorkum, Aart A. 1983. Correction of spherical aberration in charged particle lenses using aspherical foils. J. Vac. Sci. Technol. B1(4): 1312-1315.

Walther, S.R., K.N. Leung and W.B. Kunkel. 1986a. Characteristics of a compact microwave ion source. Rev. Sci. Instr. 57(8): 1531-1535. Also Lawrence Berkeley Laboratory Report LBL-20693 (Jan. 1986).

Walther, S.R., K.N. Leung, K.W. Ehlers and W.B. Kunkel. 1986b. Generation of oxygen, carbon and metallic ion beams by a compact microwave source. Presented at the Ion Implantation Technology Conference, Berkeley, CA, July 28-Aug 1, 1986. Lawrence Berkeley Laboratory Report LBL-21917.

Welch, J.J. 1984a. A low energy cyclotron for radiocarbon dating. PhD dissertation. Lawrence Berkeley Laboratory Report LBL-21255.

Welch, J.J., K.J. Bertsche, P.G. Friedman, D.E. Morris, R.A. Muller, and P.P. Tans. 1984b. A $40 \mathrm{keV}$ cyclotron for radioisotope dating. Nucl. Instr. \& Meth. B5: 230232.

Welch, J.J., K.J. Bertsche, P.G. Friedman, D.E. Morris, R.A. Muller, and P.P. Tans. 1987a. A low energy cyclotron for radiocarbon dating. Nucl. Instr. \& Meth. B18: 202-214. 
Welch, J.J., K.J. Bertsche, P.G. Friedman, D.E. Morris, and R.A. Muller. 1987b. Advanced accelerator methods: the cyclotrino. Lawrence Berkeley Laboratory Report LBL-23323. Also published in U.C. Accelerator Mass Spectrometry I, Proceedings of the First U.C. Conference on AMS. University of California, Irvine, Feb. 15, 1986. Edited by J.E. Ericson and R.E. Taylor. Livermore, Calif.: Institute of Geophysical and Planetary Physics, University of California, Lawrence Livermore National Laboratory. CONF-8602126.

Wilson, Robert R. 1938. Magnetic and electrostatic focusing in the cyclotron. Phys. Rev. 53: $408-420$.

Wollnik, Hermann. 1987. Optics of Charged Particles. Orlando: Academic Press. 